\title{
Dual Fermion Approach to Disordered Correlated Systems
}

\author{
Dissertation \\ zur Erlangung des mathematisch-naturwissenschaftlichen Doktorgrades \\ "Doctor rerum naturalium" \\ der Georg-August-Universität Göttingen \\ im Promotionsprogramm ProPhys \\ der Georg-August University School of Science (GAUSS)
}

vorgelegt von

\section{Patrick Haase}

aus Soltau

Göttingen, 2015 


\section{Betreuungsausschuss}

Prof. Dr. Thomas Pruschke,

Institut für Theoretische Physik,

Georg-August-Universität Göttingen

Prof. Dr. Reiner Kree,

Institut für Theoretische Physik,

Georg-August-Universität Göttingen

\section{Mitglieder der Prüfungskommission}

Referent: Prof. Dr. Reiner Kree, Institut für Theoretische Physik, Georg-August-Universität Göttingen

Korreferent: Prof. Dr. Thomas Pruschke, Institut für Theoretische Physik, Georg-August-Universität Göttingen

2. Korreferent: Prof. Dr. Fakher F. Assaad, Institut für Theoretische Physik und Astrophysik, Julius-Maximilians-Universität Würzburg

\section{Weitere Mitglieder der Prüfungskommission}

Prof. Dr. Karl-Henning Rehren, Institut für Theoretische Physik, Georg-August-Universität Göttingen

Prof. Dr. Laura Covi,

Institut für Theoretische Physik,

Georg-August-Universität Göttingen

Prof. Dr. Stefan Dreizler, Institut für Astrophysik,

Georg-August-Universität Göttingen

Prof. Dr. Christian Jooß,

Institut für Materialphysik,

Georg-August-Universität Göttingen

Tag der mündlichen Prüfung: 25. September 2015 
Perfectly ordered disorder designed with a helterskelter magnificence.

Emily Carr (1871-1945) 



\section{Contents}

Page

1 Introduction 1

2 Models 5

$2.1 \quad$ Interacting Systems $\ldots \ldots \ldots \ldots \ldots$. . . . . . . . . . . 5

2.1 .1 The Hubbard Model . . . . . . . . . . . . . . . . . . . . . . . 7

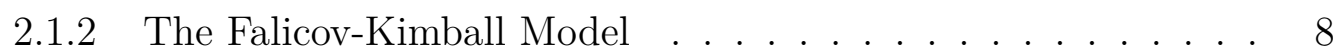

2.2 Disordered Systems $\ldots \ldots \ldots$. . . . . . . . . . . . . . . . . 9 9

2.2 .1 The Anderson Disorder Models . . . . . . . . . . . . . . . . . 11

2.2 .2 Model for the Disorder Potential . . . . . . . . . . . . . . . . 12

2.2 .3 Modeling Disorder in Macroscopic Systems . . . . . . . . . . . 13

2.3 Summary . . . . . . . . . . . . . . . . . . . . 16

3 Conventional Mean-Field Methods for Correlated Lattice Models 17

$3.1 \quad$ Dynamical Mean-Field Theory . . . . . . . . . . . . . . . . . . . . . 18

3.1.1 Self-Consistent Description of the Local Green Function. . . . . 19

3.1.2 Alternative Disorder-Average: The Typical-Medium-Theory . 21

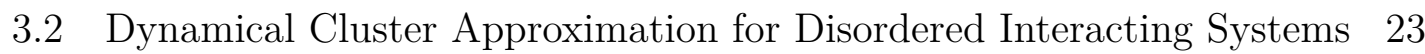

3.2.1 Self-Consistent Description of the Cluster Green Function . . 25

3.2 .2 Changes to the Impurity Solver for Clean Systems . . . . . . . 27

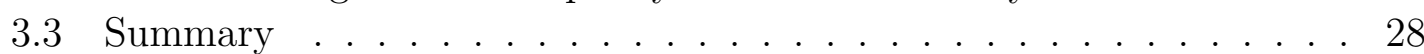

4 The Dual Fermion Approach for Disordered Interacting Systems 29

4.1 Derivation of the Formalism . . . . . . . . . . . . . . . . 30

4.2 Perturbation Theory for the Self-Energy . . . . . . . . . . . . . . . . 41

$4.2 .1 \quad$ Replica Limit and Feynman Rules for Self-Energy Diagrams . 41

4.2 .2 First Order and Second Order Contributions . . . . . . . . . . 46

4.2 .3 The FLEX Approximation . . . . . . . . . . . . . . . . 50

4.3 Relations between Dual and Real Quantities . . . . . . . . . . . . . . 52

4.4 Self-Consistency in the Dual Fermion Approach . . . . . . . . . . . . 56

$4.5 \quad$ Observable of Interest: Conductivity $\ldots . . . . . . .59$

4.5 .1 Horizontal Channel . . . . . . . . . . . . . . . . . . . . . 60 . . . . . . 60

4.5 .2 Vertical Channel . . . . . . . . . . . . . . . . . 66

4.5 .3 Maximally Crossed Channel . . . . . . . . . . . . . . . . 67 
4.6 Summary $\ldots \ldots \ldots \ldots \ldots$

5 Quantum Monte Carlo for Impurity Problems 71

5.1 Basic Ideas of the Monte Carlo Method . . . . . . . . . . . . . . . . . 72

5.2 The Interaction Expansion . . . . . . . . . . . . . . . . . . . 75

5.2 .1 Monte Carlo Weights and the Random Walk . . . . . . . . . . 75

$5.2 .2 \quad$ Single-Particle Measurements . . . . . . . . . . . . . . 78

$5.2 .3 \quad$ Two-Particle Measurements . . . . . . . . . . . . . . . . . . . 78

5.2 .4 Disorder-Averaged Quantities from Monte Carlo Simulations . 80

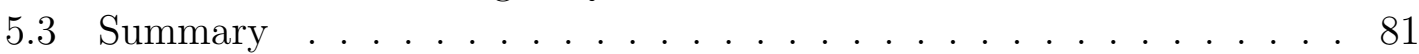

6 Test Case for the Dual Fermion Algorithm: The Anderson-FalicovKimball Model $\quad \mathbf{8 5}$

6.1 Dual Fermion Corrections in 1d . . . . . . . . . . . . . . . . . . 86

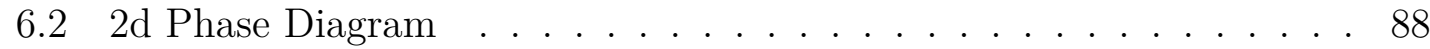

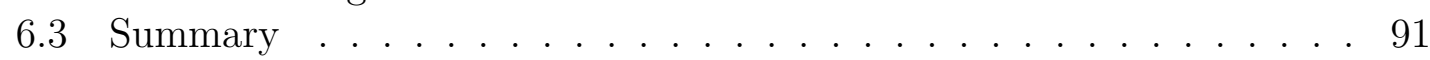

7 Results for the Anderson-Hubbard Model 95

7.1 1d Results . . . . . . . . . . . . . . . . . . . 96

7.2 3d Results . . . . . . . . . . . . . . . . . . . . . . . . . 100

$7.2 .1 \quad$ Antiferromagnetic Transition . . . . . . . . . . . . . 100

$7.2 .2 \quad$ Mott Metal-Insulator Transition . . . . . . . . . . . . . . . . . 103

7.2 .3 Anderson Localization and Phase Diagram . . . . . . . . . . . 106

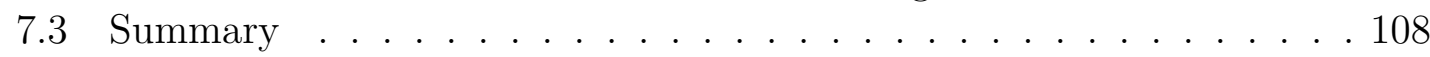

8 Summary and Perspective 111

\begin{tabular}{ll}
\hline Appendices & 115
\end{tabular}

\begin{tabular}{|l|l|}
\hline A The Sign Problem in Quantum Monte Carlo Simulations & 117
\end{tabular}

\begin{tabular}{lll}
\hline B & Fast Matrix Updates for Quantum Monte Carlo & 119
\end{tabular}

\begin{tabular}{|lll}
\hline C Dual Fermion Mapping for the Anderson-Falicov-Kimball Model & 121
\end{tabular}

\begin{tabular}{ll}
\hline Two-Particle Green Functions & $\mathbf{1 2 5}$
\end{tabular}

D.1 Channels . . . . . . . . . . . . . . . . . . . . . . . . . . . . . . . . . . . 127

D.2 Vertices . . . . . . . . . . . . . . . . . . . . . . . . . . . 129

E Comment on the Use of the Replica Trick 131

\begin{tabular}{ll}
\hline Bibliography & 133
\end{tabular} 


\section{Introduction}

Solid state physics is situated at the boundary between the microscopic and macroscopic world. The classical sample of interest is a cube of dimension $1 \mathrm{~cm}^{3}$ containing $6 \cdot 10^{23}$ particles. This imaginary object is of tangible size, still many of its properties are governed by microscopic laws of physics, namely quantum mechanics. Electrons and atoms determine the properties of the sample, Coulomb interaction and Pauli principle are the driving forces. Gravitation, paramount in the macroscopic world, and weak and strong interaction, the forces of the subatomic world of the nuclei of the atoms, are mostly irrelevant for the physics of the solid. Thus, the stage is set.

Quantum mechanics provides a clear description for every system composed of electrons and atoms, in principle allowing for an exact solution of the problem. The large number of particles, however, renders an exact solution impossible in almost all cases. This is true for classical systems and even more so for quantum mechanical systems, due to the exponential growth of the Hilbert space.

Two approaches exist to overcome this obstacle. The first is the simplification of the Schrödinger equation. Instead of solving the Hamiltonian involving all degrees of freedom of the system, simplifications are introduced to reduce the number of degrees of freedom. To this end, degrees of freedom and interactions that are deemed irrelevant for the problem at hand are removed and, if necessary, are replaced by effective interactions. An example is the neglect of atomic motion, known as the Born-Oppenheimer approximation [1], and the coupling of the electrons to the atomic degrees of freedom. If the coupling of the electrons to the atoms cannot be neglected, the concept of elementary lattice vibrations, so-called phonons, can be introduced along with an effective interaction between electrons and phonons.

The second approach consists in the development of new methods that are particularly well suited for a given problem, exploiting symmetries and special properties that are unique for a given situation. Symmetries can include translational and rotational invariance. Special properties can be a small entanglement entropy in one dimension, that is exploited for example in the density matrix renormalization group [2]. Another example is nearly-free electron behavior in many metals, where Fermi liquid theory [3, 4] can be applied successfully. Other approaches rely on an expansion around a known solution in terms of a small parameter. These approaches are known as perturbation theory [5]. An approach can be analytical, i. e. a pen-and-paper solution, purely numerical, e.g. a straight forward diagonalization of the Hamiltonian by means of numerical algorithms, or a combination of both.

In general, it is not enough to use one of the two approaches above. A successful 
solution for a given problem often requires to find an effective, minimal model that covers all relevant aspects of the problem and then find a method that is suitable for solving the model. Usually, this requires some analytical work. This can lead to a satisfactory solution of the problem or be the starting point for numerical methods.

In this thesis we are interested in the situation of strongly correlated electron systems with disorder, where disorder refers to defects in the crystal and strongly correlated means that electrons strongly repel each other via the Coulomb interaction. Materials with strongly correlated electrons include transition-metal oxids [6] and heavy fermions [7, 8, 9, 10, 11]. They are interesting because of their rich phase diagram [12, 13] and are promising candidates for applications [14, e.g. smart windows using $\mathrm{VO}_{2}$ [15].

Disorder is often neglected in effective models, but to a certain degree, disorder in the form of impurities, vacancies, dislocations, etc. is present in all systems at finite temperature. Hence, it is necessary, to study the effects of disorder and see how it affects the physical properties. Strong disorder can even be the dominant factor, e.g. in the event of a metal-insulator transition known as Anderson localization [16]. Close to the Anderson transition from the metal to the Anderson localized state the wave function takes on a multifractal nature [17, 18], highlighting the complex behavior associated with disorder. Even in strongly correlated electron systems, the impact of disorder on transport and thermodynamic properties is often not negligible [19, 20].

An important model for disordered interacting systems is the disordered Hubbard model [21, 22, 23], also known as the Anderson-Hubbard model [24]. It is a lattice model for electrons that can hop from one site to another. The electrons can have up or down spin and interact via a repulsive Coulomb interaction if two electrons occupy the same site. Disorder is taken into account by a local random potential that equally affects up and down electrons. In the model, electrons obey the Pauli principle, i.e. only electrons of different spins can occupy the same site. There is only one orbital in the simplest form of the model. This is an oversimplification that is only justified, if one is interested in general properties of the Hamiltonian. For applications to real materials the single orbital model is not suited.

The Anderson-Hubbard model is a seemingly simple model, but even the Hubbard part alone, i. e. hopping and interaction, is hard to solve. Generally, quantum Monte Carlo [25] can be used, but it only allows to treat small systems, far away from the thermodynamic limit. Also, away from half-filling it suffers from the infamous sign problem [25], which means that the computational cost scales exponentially with system size and inverse temperature. This is believed to be a manifestation of the exponential growth of the Hilbert space.

In 1989 Metzner and Vollhardt introduced dynamical mean-field theory (DMFT) [26, 27, 28, 29] that allows to treat interacting systems in the thermodynamic limit and is exact in infinite dimensions. It was extended in [30, 31] to treat disordered 
interacting systems. DMFT treats local correlations exactly by mapping the lattice problem to an impurity problem that is solved self-consistently, but DMFT ignores non-local correlations altogether when employed for finite-dimensional systems. This is an approximation away from the limit of infinite dimensions and it fails when non-local correlations become important. Unlike quantum Monte Carlo for lattice models, it does not suffer from the sign problem for a single orbital model.

Cluster extensions of DMFT are the dynamical cluster approximation (DCA) [32, 33] and cellular DMFT [34]. For the Hubbard model both methods often use quantum Monte Carlo to solve the cluster problem and thus indirectly suffer from the sign problem.

The methods mentioned so far are either not working in the thermodynamic limit, neglect non-local correlations, or suffer from the sign problem. A possible way out are diagrammatic extensions of DMFT [35] that rely on an impurity problem to capture the local physics and treat non-local correlations using perturbation theory. Two such methods for purely interacting systems are the dual fermion approach [36, 37] and the dynamical vertex approximation [38, 39]. Prior to this work, the dual fermion approach has also been applied to purely disordered systems [40] as well.

In this thesis, we want to combine the two dual fermion approaches for disordered and interacting systems to a method that treats both disorder and interaction on equal footing. We develop the formalism for the Anderson-Hubbard model and Anderson-Falicov-Kimball model [41, which is a simplified version of the AndersonHubbard model. Both models are introduced and discussed in chapter 2 .

We are interested in the case of macroscopic systems. In this context a macroscopic system is a system that is much larger than the coherence length of the electrons. In general, such systems are too large to be simulated directly. Thence, we exploit the self-averaging property of electrons and use disorder-averaging. This allows to model a macroscopic system by a set of smaller subsystems that can be simulated, and physical properties of the original system are calculated as the average of the corresponding properties of the subsystems. A drawback of the disorder-average is that it is not suited for an accurate treatment of Anderson localization, meaning results around the critical disorder strength have to be taken with care.

In chapter 3 we introduce DMFT and DCA for disordered interacting systems. DMFT is the method we want to improve and DCA, an established cluster method, acts as a benchmark for our approach.

Chapter 4 is the main result of this thesis. We present a detailed discussion of the dual fermion approach for disordered interacting systems. This includes a discussion of the perturbative treatment of the dual single-particle Green function and the selfconsistency loop. The chapter ends with the calculation of vertex corrections for the conductivity, which is used as an example to show how the dual fermion approach can be used to calculate two-particle quantities. 
DMFT, DCA and the dual fermion approach require the solution of an impurity or cluster problem. In all three cases quantum Monte Carlo can be used. The interaction expansion algorithm [42, 43, 44] is discussed in chapter 5, along with modifications needed for the inclusion of disorder.

Chapters 6 and 7 present results for the Anderson-Falicov-Kimball model and the Anderson-Hubbard model. The Anderson-Falicov-Kimball model serves as a test case, therefore we limit our studies to the $1 \mathrm{~d}$ and $2 \mathrm{~d}$ system. We compare the dual fermion approach to DCA in $1 \mathrm{~d}$ and obtain a phase diagram in $2 \mathrm{~d}$ as a function of interaction and disorder strength.

The discussion of the Anderson-Hubbard model starts with a comparison between dual fermions and DCA in 1d, as well. After that, we turn to the $3 \mathrm{~d}$ case and investigate phase transitions, namely the antiferromagnetic transition, Mott transition and Anderson transition. Prominent 3d materials with interactions and disorder are alloys like brass, bronze or cast iron.

Throughout this thesis we set $\hbar=k_{B}=1$, where $\hbar$ is the reduced Planck constant and $k_{B}$ is the Boltzmann constant. A quantity is regarded as finite if it is less than infinity and non-zero. 


\section{Models}

The purpose of this chapter is to introduce two models for disordered interacting systems. It is organized as follows.

First, in section 2.1 some phenomena in interacting systems will be presented and the Hamiltonian for the Hubbard model [21, 22, 23] will be introduced, as well as the Hamiltonian for the Falicov-Kimball model [45]. The Hubbard model is a lattice model that describes two types of electrons that repel each other if they occupy the same site. The Falicov-Kimball model is similar, except only one electron species is mobile, the other is localized.

Second, in section 2.2 concepts for disordered systems will be introduced along with some phenomena caused by disorder. The Hamiltonians of the AndersonHubbard model and Anderson-Falicov-Kimball model for disordered-interacting systems will be introduced along with a discussion of the disorder potential in section 2.2.2 Section 2.2.3 concludes this chapter with a discussion of the self-averaging property of electrons and we supplement the Hamiltonians given in section 2.2 with the disorder-average as the final ingredient for the disorder models for macroscopic systems.

\subsection{Interacting Systems}

It was a common practice in the early days of condensed matter theory to ignore the effects of electron-electron interactions entirely or just use a mean-field approximation, e.g. the Hartree-Fock method [46, 47]. Surprisingly enough, this yielded good qualitative as well as quantitative results for some materials and led to the invention of band theory [48]. Band theory led to an understanding of the mechanisms that make a material a metal, insulator or semiconductor. Unfortunately, one of its predictions, namely that a material with one free electron per unit cell is a metal was proved wrong by experiment for several materials including transition metal oxides [6] such as $\mathrm{V}_{2} \mathrm{O}_{3}$. In these materials strong repulsive Coulomb interactions prevent electrons from hopping to neighboring sites and thereby localize them. These type of materials are called Mott insulators [49]. An additional effect that is observed in $\mathrm{V}_{2} \mathrm{O}_{3}$ is the formation of an antiferromagnetic state due to the local Coulomb repulsion [13]. The antiferromagnetic state allows for virtual hopping processes which lower the energy [50]. A phase diagram for $\mathrm{V}_{2} \mathrm{O}_{3}$ at various levels of doping is shown in fig. 2.1. 


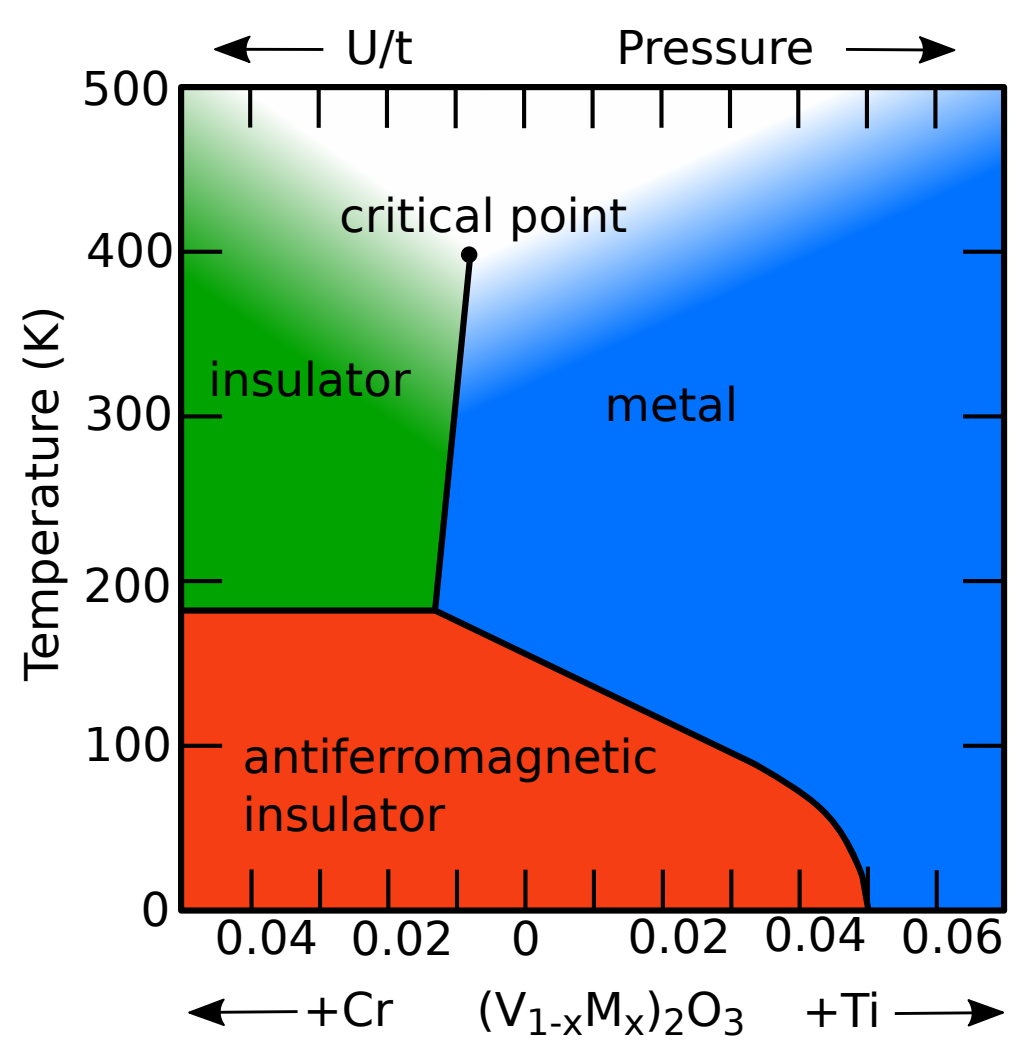

Figure 2.1: The schematic phase diagram of $\mathrm{V}_{2} \mathrm{O}_{3}$ based on the data of [13]. The phase diagram shows three different phases that can be explained by the Hubbard model. The metal for high temperatures and weak interactions is dominated by the kinetic energy term in the Hamiltonian. Upon increasing the pressure via doping, $\mathrm{V}_{2} \mathrm{O}_{3}$ becomes a Mott insulator, dominated by the local Coulomb repulsion. For low temperatures and sufficient pressure, the material enters an antiferromagnetic state, caused by the interplay between both the kinetic and potential energy terms in the Hamiltonian. The Coulomb repulsion prevents double occupancies, but the antiferromagnetic order allows for virtual hopping, which lowers the energy.

Another effect of electron-electron interactions is the formation of Fermi liquids [3]. Landau introduced the idea of a Fermi liquid in his seminal paper in 1956 [4]. The Fermi liquid is adiabatically connected to the free electron gas. While the excited states of the free electron gas are stable, the excited states of the Fermi liquid have a finite, yet long life time. For many purposes, their longevity allows to treat them like elementary particles with a renormalized mass. These excitations are called quasiparticles.

The Fermi liquid state can be regarded as the ordinary metallic state. In phase diagrams for strongly interacting materials, the metallic phase is often separated into a Fermi liquid regime and a non-Fermi liquid regime. Non-Fermi liquid is quite 
unspecific, e.g. it can mean that exponents of thermodynamic quantities stray from the Fermi liquid value. An example is $\mathrm{Y}_{1-x} \mathrm{U}_{x} \mathrm{Pd}_{3}$ [51], where the exponent for the temperature dependence of the resistivity was found to be $n \approx 1.13 \pm 0.04$, whereas a Fermi liquid has an exponent $n=2$. Another example is the transition from a Fermi liquid to a Luttinger liquid upon a dimensional crossover from $2 \mathrm{~d}$ to $1 \mathrm{~d}$ [52].

In some materials the renormalized mass can be up to $10^{3}$ electron masses. Materials with this property are called Heavy Fermion compounds [7, 8, 9, 10, 11] if rare-earth elements like $\mathrm{Ce}, \mathrm{Yb}$ or $\mathrm{Y}$ or actinides like $\mathrm{U}$ are involved. These materials have a partially occupied $4 f / 5 f$ shell as well as a conduction band made of other orbitals which may hybridize with the $f$-bands. The strong spatial confinement of the $f$ orbitals gives rise to a strong local Coulomb repulsion which is so strong that double occupancy is prohibited and the $f$-electrons act as isolated spins. These spins can couple to the spins of the conduction electrons and cause a variety of phenomena, for example the Kondo effect [53] if the concentration of magnetic moments is small. One model used to describe Heavy Fermion systems is the Kondo lattice model [54, 55].

\subsubsection{The Hubbard Model}

In this thesis we will investigate systems with Coulomb interactions that are strong but still allow for double occupancy. This is the case for transition metals like $\mathrm{Ni}, \mathrm{Fe}, \mathrm{V}$, Co and $\mathrm{Mn}$ as well as their oxides. Transition metals have partially filled $d$-shells [49]. The $d$-orbitals are relatively narrow, such that the repulsion between electrons in the same orbital is strong but double occupancy is still possible. A common model for these materials is the Hubbard model [21, 22, 23] with the Hamiltonian

$$
H_{H u b b}=-\sum_{i j, \sigma}\left(t_{i j, \sigma}+\mu_{e f f} \delta_{i j}\right)\left(c_{\sigma i}^{\dagger} c_{\sigma j}+h . c .\right)+U \sum_{i}\left(n_{i \uparrow}-\frac{1}{2}\right)\left(n_{i \downarrow}-\frac{1}{2}\right) .
$$

Here, $i$ and $j$ denote site indices, $\sigma=\uparrow, \downarrow$ is the spin index, $c_{\sigma i}^{(\dagger)}$ annihilates (creates) an electron of spin $\sigma$ at site $i, n_{\sigma i}=c_{\sigma i}^{\dagger} c_{\sigma i}$ is the particle number operator for electrons of spin $\sigma$ at site $i$. $U$ parametrizes the local Coulomb repulsion, $\mu_{\text {eff }}=$ $\mu-\frac{U}{2}$ is the effective chemical potential and $\mu$ is the chemical potential. For halffilling $\mu_{e f f}$ is zero. $t_{i j, \sigma}$ is the hopping amplitude for an electron with spin $\sigma$ and only depends on the difference $|i-j|$ between sites $i$ and $j$ because a translationally invariant lattice is assumed. In the Hubbard model the spin dependence of the hopping amplitude is usually dropped. The Hamiltonian in eq. 2.1 neglects nonlocal Coulomb interactions. This is a simplification that is justified if the screening of the Coulomb interaction in the material is strong. In this simple form, the Hubbard model has only one orbital.

Overall, the Hubbard model is an oversimplification if quantitative predictions for 
experiments are required. Although this Hamiltonian is very simple, too simple to model real materials, no one has succeeded in finding a general analytic solution in more than one [56] and less than $\infty$ [26] dimensions. Despite these two problems, the Hubbard model is very popular. It was successfully applied to study the Mott metal-insulator transition as well as itinerant magnetism, where it provides a simple environment [57]. In these studies the simplicity of the model can be an advantage as it allows to determine more easily the physical origin, hopping or local Coulomb repulsion, of observed features. Compared to models with more parameters and thus more possible origins this is a clear benefit.

Although no general analytic solution exists, a lot of progress in understanding this model has been made [58]. Besides analytic treatment of the large and small $U$ limit, numerical methods shed some light on the underlying physics [59]. Such methods include exact diagonalization (ED) [60, quantum Monte Carlo (QMC) [25], dynamical mean-field theory [26, 27, 28, 29] and non-local extensions thereof. These include the dynamical cluster approximation (DCA) [32, 33, 61], cellular DMFT (CDMFT) [34] or the dual fermion approach [42, 43]. The dual fermion approach is used and developed further in this thesis.

\subsubsection{The Falicov-Kimball Model}

Another model for rare-earth and transition metal materials is the Falicov-Kimball model [45]. It can be regarded as a simplified Hubbard model and is obtained from the Hubbard model by letting $t_{i j, \sigma} \rightarrow 0$ for one spin degree of freedom. Now, there exist two types of fermionic particles that are fundamentally different. One species is immobile and is distributed on the lattice according to a thermal distribution. These particles are called $f$ electrons, because of the $f$ orbitals in rare-earth elements that the model was developed for. The other species is mobile and can hop from one site to another, depending on the hopping-elements $t_{i j}$. These particles are called conduction electrons or just $c$ electrons. Due to their fermionic nature, a site cannot be occupied by more than one $c$ or $f$ electron. If both types of electrons occupy the same site, they will experience a repulsive interaction $U$. The corresponding Hamiltonian reads

$$
H_{F K}=\sum_{k}\left(\epsilon_{k}-\mu\right) c_{k}^{\dagger} c_{k}+U \sum_{i} n_{i}^{c} n_{i}^{f}
$$

where $\mu$ is the chemical potential, $c_{k}^{(\dagger)}$ annihilates (creates) a $c$ electron of momentum $k$ and $n_{i}^{c / f}$ measures the density of $c / f$ electrons at site $i$. The kinetic part has been Fourier transformed and has been written in terms of the dispersion relation $\epsilon_{k}$.

The Falicov-Kimball model is the simplest model that displays long-range order [62, 63, 64]. However, the simplicity comes at a price, for example in combination with the coherent potential approximation [65, 66] it only yields a crossover for the metal-insulator transition [67]. 
In this thesis we opted to use the Falicov-Kimball model as a test case. The impurity problem that one has to solve in dynamical mean-field theory and the dual fermion approach is particularly simple for the Falicov-Kimball model and thus testing numerical algorithms for this model is fast.

\subsection{Disordered Systems}

In many physical theories of the solid a perfect lattice or crystal is assumed. This leads to translational invariance with respect to a shift by a lattice vector and the conservation of crystal momentum. In a lattice, electronic momentum $k$ is not fully conserved but only up to a reciprocal lattice vector

$$
k=\tilde{k}+G
$$

where the crystal momentum $\tilde{k}$ is a vector in the first Brillouin zone and here $G$ is a reciprocal lattice vector. This facilitates analytic as well as numerical calculations. However, it completely ignores that no crystal is ever perfect, which follows from entropy based arguments.

In this thesis, we want to go beyond the perfect crystal. Instead, we will take into account that there will always exist a number of defects in the group of atoms forming the lattice or crystal. A few possible deviations from the perfect crystal are illustrated in fig. 2.2.

Whatever it is, the potential and hopping parameters around those defects will be changed and depending on the nature of those defects and their concentration the behavior of a sample may change as well. A famous example is the Kondo effect [53], where a low concentration of magnetic impurities in a metal may cause an increase in resistivity at low temperatures. Another effect of introducing defects in a crystal is visible in electron diffraction. Here, the clear peaks in the diffraction pattern of the clean sample begin to smear out when disorder starts to break the translational invariance and long-range order of the crystal [68, 69]. Arguable the most prominent influence of disorder is its diminishing effect on the conductivity which can localize electrons entirely. This effect is known as Anderson localization [16] and it is caused by coherent backscattering of electrons from impurities. If the backscattering does not lead to localization but only to a reduction of the conductivity it is called weak localization [70].

Before we move on, let us mention a few effects where disorder and interaction act together. As was presented above, both interaction and disorder can lead to localization and insulating behavior [16, 49]. For a long time it was believed that the ground state of a $2 \mathrm{~d}$ system is always insulating if one has disorder or interaction. This does not hold if both are present [71, 72, 73]. In this case, a metallic ground state is possible. 


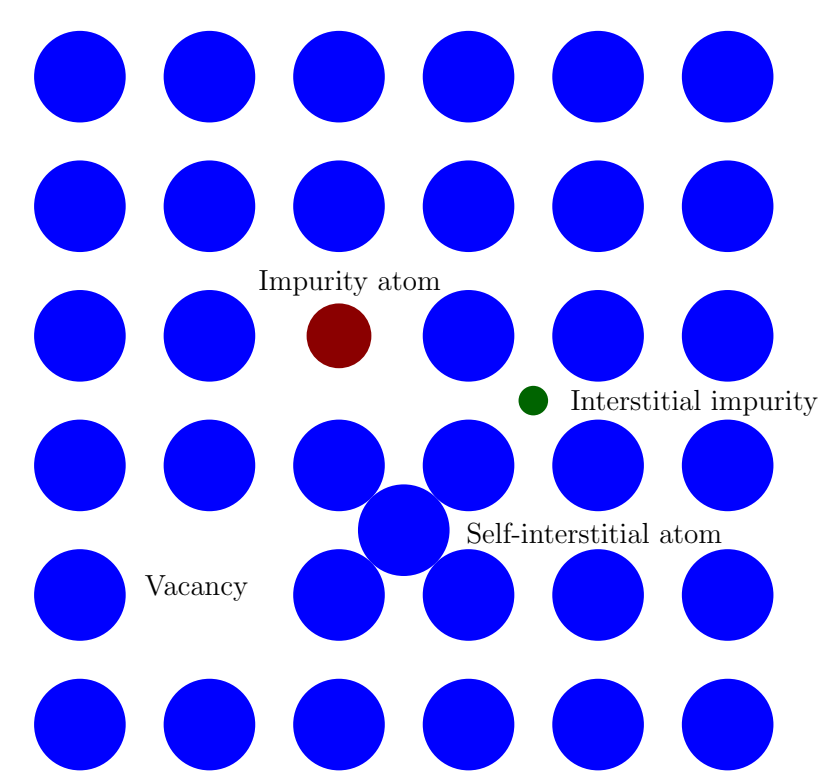

Figure 2.2: Possible deviations from the perfect crystal. An atom can be replaced by an impurity atom or be removed from its position and leave a vacancy. It is also possible to have atoms that do not occupy a lattice site. This can be an interstitial impurity as well as a self-interstitial atom, which is a displaced atom from the regular lattice.

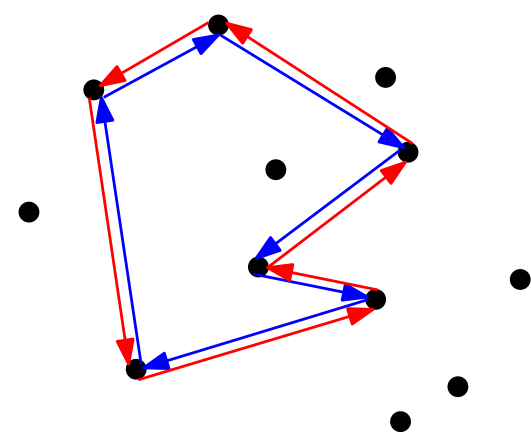

Figure 2.3: Anderson localization is caused by coherent backscattering of electrons from the randomly distributed impurities. The impurities give rise to a random potential throughout the crystal. 
An interesting example of the influence of disorder is the superconducting state in thin films. As has been observed in FeSe, the superconducting critical temperature $T_{c}$ in thin films is increased by a factor of nearly ten [74]. On the other hand, decreasing the film thickness is regarded as a way to introduce disorder in the system [75, 76] and in effect the critical temperature is reduced, e.g. in [75] it was found that increasing amounts of disorder can change the temperature dependence of the resistivity in thin films of $\mathrm{NbN}$ from a metal $\left(\frac{d \rho}{d T}>0\right)$ to that of an insulator $\left(\frac{d \rho}{d T}<0\right)$. However, the transition to a superconductor survived, albeit moved to lower temperatures. $\mathrm{Nb}$ is a transition metal, thus electron-electron interactions can be assumed to play a crucial role. The amount of disorder was tuned by the thickness of the sample. In [77] disorder in the samples was tuned by annealing and this also led to a decrease in $T_{c}$.

The interplay of the increase of $T_{c}$ in thin films with the effects of disorder make these kind of systems worth studying, for example a bulk superconductor with $T_{c, \text { Bulk }}=30 \mathrm{~K}$ would give a $T_{c}$ at room temperature if a similar increase in thin films could be realized.

An effect that we will study in section 7.2.1 is that disorder can also increase the Néel temperature for strongly interacting systems, which was shown in [24].

\subsubsection{The Anderson Disorder Models}

The models used in this thesis for disordered interacting systems are based on the Hubbard model and Falicov-Kimball model. A general extension of the Hubbard Hamiltonian for an interacting disordered system will take a form like

$$
H_{H u b b, D i s}=H_{H u b b}-\sum_{i, \sigma} v_{i \sigma} n_{i \sigma} .
$$

In the disordered system the hopping $t_{i j, \sigma}$ depends on the two individual sites involved and $v_{i \sigma}$ is a random site-dependent potential, also called the disorder potential. It may be spin-dependent. Finally, the Hubbard interaction $U$ could become a site-dependent random variable as well.

These modifications make for a quite complex Hamiltonian. The explicit sitedependence of the hopping is not very well suited for an approach in momentum space. In this thesis it is assumed that the hopping and disorder potential do not depend on spin, i.e. the energy of the impurities does not depend on whether an electron with up or down spin is added. We will restrict ourselves to the disordered potential and neglect the influence of the potential on the hopping. This is called diagonal disorder. We obtain the Anderson-Hubbard Hamiltonian

$$
H_{A H}=-\sum_{i j, \sigma}\left(t_{i j}+\mu \delta_{i j}\right)\left(c_{\sigma i}^{\dagger} c_{\sigma j}+h . c .\right)-\sum_{i, \sigma} v_{i} n_{i \sigma}+U \sum_{i}\left(n_{i \uparrow}-\frac{1}{2}\right)\left(n_{i \downarrow}-\frac{1}{2}\right)
$$


for interacting disordered systems. For $v_{i} \equiv 0$ we recover the Hubbard model. We will also refer to this situation as the clean system. Throughout this thesis, the Anderson model [16] refers to this Hamiltonian for $U=0$. It is not to be confused with the single impurity Anderson model [78] or the periodic Anderson model [79].

Analogous to the Hubbard model, the Falicov-Kimball model is supplemented by a disorder potential. The random potential $v_{i}$, that was introduced in eq. (2.5), is added to $H_{F K}$. It will act on the $c$ electrons only. The Hamiltonian for the model reads

$$
H_{A F K}=H_{F K}-\sum_{i} v_{i} n_{i}^{c}
$$

This model is called Anderson-Falicov-Kimball model [41] and it serves as a first test ground for the dual fermion method that we developed and that is introduced in chapter 4 .

\subsubsection{Model for the Disorder Potential}

In the following, we will examine how the potential $v_{i}$ can be constructed for both models [70]. To this end, we introduce $N_{i m p}$ impurities that are randomly distributed on the lattice. The potential at $r$ will be

$$
V(r)=\sum_{j}^{N_{i m p}} u\left(r-P_{j}\right),
$$

where $P_{j}$ is the position of the $j$ th impurity. In a solid, an electrical charge is screened with a screening length $a$. The Coulomb potential thus becomes a Yukawa potential [80] $u(r)=-\frac{e_{0}^{2}}{|r|} e^{-\frac{|r|}{a}}$. The first approximation that is made is to neglect the spatial extend of an atom and set $v_{i}=V\left(r_{i}\right)$ where $r_{i}$ is the position of the $i$ th atom. This means the potential is assumed to be constant on the scale of an atom.

The potential will look quite random as the impurities are randomly distributed, but due to the positions of the impurities the values of the random potential on neighboring sites will still be correlated. This will be ignored to facilitate the modeling and make it more general. Instead, a truly random potential is chosen for each site, uncorrelated with its neighbors.

In this thesis two different disorder distributions are used. The first is binary disorder with the distribution function

$$
p_{\text {Bin }}\left(v_{i}\right)=\frac{1}{2}\left[\delta\left(v_{i}-\frac{V}{2}\right)+\delta\left(v_{i}+\frac{V}{2}\right)\right] .
$$

The second is box disorder with a continuous distribution function

$$
p_{\text {Box }}\left(v_{i}\right)=\frac{1}{V} \Theta\left(\frac{V}{2}-\left|v_{i}\right|\right) \text {. }
$$


$\Theta$ is the Heaviside function

$$
\Theta(x)=\left\{\begin{array}{l}
0 \text { if } x<0 \\
1 \text { if } x \geq 0
\end{array} .\right.
$$

$V$ defines the strength of the disorder in both distributions. The Hamiltonians 2.5 and 2.6 in general lack translational invariance as mentioned above, thus crystal momentum is not conserved and single-particle observables depend on two momenta.

The following section expands on the disorder term in our models and how it influences the comparison with experiments, especially for intermediate-sized and macroscopic systems. Here, a system of macroscopic size is a system that is much larger than the coherence length of the electrons, and an intermediate-sized system ranges from about hundred to a thousand atoms to the macroscopic system. It will become obvious that there are severe problems for systems of intermediate size.

At the level of macroscopic systems the self-averaging effect helps to overcome this problem by introducing a disorder-average. It provides a solution of the problem that allows to make general statements about macroscopic disordered systems and it introduces a pseudo-momentum conservation such that $O_{k k^{\prime}} \propto \delta_{k k^{\prime}}$ for a singleparticle observable $O$.

\subsubsection{Modeling Disorder in Macroscopic Systems}

The models described in eqs. (2.5) and (2.6) share the random potential term. A priori it is unclear what one should do with this term. A four-site system with binary disorder can be used to illustrate this point. There are $2^{4}=16$ possible configurations for this system. The properties of this system will depend strongly on the realization of the disorder, e.g. the system with all four local potentials equal to $V / 2$ will be very different from the system with all four local potentials equal to $-V / 2$.

As a consequence, one realization of the disorder will not be enough to make general statements about systems with a given disorder distribution. In fact, for small systems as just described one can only make statements for specific realizations. This complicates comparison with experiments as experimentalists would have to gain full knowledge about their sample such that it can be simulated. Also, experimental results should most often be reproducible not just for one sample, but for all samples that can be considered similar for a given experiment. For intermediatesized systems, the situation becomes even worse as a given system may be too large to simulate in its entirety and translational invariance cannot be exploited for approximations. The bottom line is, the Hamiltonians given in eqs. 2.5 and 2.6 can only be applied directly to very small systems if comparison with experiments is the goal. This would make the use of these Hamiltonians pointless for macroscopic systems if there were not the effect of self-averaging. 
Self-averaging occurs for electrons traveling in a disordered system if the coherence length of the electron is much smaller than the sample size. The conductivity shall serve as an example. If the coherence length is long, backscattering of different impurities can lead to interfering paths of the electron. This in turn can lead to weak localization and thus to a reduced conductivity or even to Anderson localization and to a vanishing conductivity. If the coherence length is short, the scattering from multiple impurities will not lead to interference on larger length scales and Anderson localization is not possible. The conductivity may still be reduced but the effect is weaker.

What happens in a macroscopic sample? As the electron is influenced by interference effects only in the region defined by its coherence length, we can decompose the sample into subsystems. Under the assumption that no macroscopic regions exist in the sample that are either insulating or superconducting and can thus dominate the conductance, the conductivity of the sample will be the average over the partial conductivities of those subsystems. If we further assume that these subsystems are themselves large compared to the microscopic scales, they may each be viewed as independent realizations of disorder, hence the previous average can also be viewed as an average over different realizations of disorder. This is the self-averaging effect.

The self-averaging effect can be incorporated in the disorder models described above by imposing an averaging procedure for the evaluation of observables. In this thesis the procedure is referred to as disorder-averaging. To this end, the replica trick is used. As observables are calculated from the free energy $F=-T \ln Z$, the free energy and thus $\ln Z$ have to be averaged. $Z$ is the partition function and $T$ the temperature. The replica trick [81, 82]

$$
\{\ln Z\}=\lim _{m \rightarrow 0} \frac{\left\{Z^{m}\right\}-1}{m}
$$

allows to integrate out the disorder analytically and thus restore pseudo-momentum conservation. $\{\ldots\}=\int d v p(v) \ldots$ denotes the disorder-average according to a probability density $p$ for the disorder. Equation 2.11 follows from

$$
\begin{aligned}
\ln x & =\int_{1}^{x} d t \frac{1}{t} \\
& =\lim _{m \rightarrow 0} \int_{1}^{x} d t \frac{1}{t^{1-m}} \\
& =\lim _{m \rightarrow 0} \frac{x^{m}-1}{m} .
\end{aligned}
$$

$m$ is taken to be an integer and it is assumed that in the end the limit $m \rightarrow 0$ can be performed. Within the replica trick one has to deal with $m$ replicas of $Z$ instead of $\{\ln Z\}$. This facilitates analytic calculations and is a standard trick used for disordered systems as well as spin-glass systems [83]. 
Using the replica trick, $\{\ln Z\}$ becomes

$$
\begin{aligned}
\{\ln Z\} & =\int \prod_{i} d v_{i} p\left(v_{i}\right) \ln Z^{v} \\
& =\lim _{m \rightarrow 0}\left(\frac{1}{m} \int \prod_{i} d v_{i} p\left(v_{i}\right) \int \prod_{\alpha} D\left[c^{\alpha}, \bar{c}^{\alpha}\right] e^{-S_{R}^{v}}-1\right),
\end{aligned}
$$

where

$$
S_{R}^{v}=-\sum_{\alpha} \sum_{\omega k \sigma} \bar{c}_{\omega k \sigma}^{\alpha}\left(\omega+\mu-\epsilon_{k}\right) c_{\omega k \sigma}^{\alpha}-\sum_{i, \alpha} \int_{0}^{\beta} d \tau v_{i} n_{i}^{\alpha}(\tau)+\sum_{i, \alpha} \int_{0}^{\beta} d \tau U n_{\uparrow i}^{\alpha}(\tau) n_{\downarrow i}^{\alpha}(\tau)
$$

is the action for a given disorder configuration and $Z^{v}$ is the corresponding partition function. $\alpha$ is a replica index. In the above, the replica limit introduced $\lim _{m \rightarrow 0} \frac{-1}{m}$. This terms is a constant with respect to derivatives of the partition function and thus it can be ignored. As such derivatives are performed before the replica limit, there will be no problem with $\frac{1}{0}$. Now, the disorder can be integrated out analytically [84]:

$$
\int d v_{i} p\left(v_{i}\right) e^{v_{i} \sum_{\alpha} \int d \tau n_{i}^{\alpha}(\tau)}=e^{W\left(\tilde{n}_{i}\right)}
$$

with

$$
W\left(\tilde{n}_{i}\right)=\sum_{l=2}^{\infty} \frac{1}{l !}\left\langle v_{i}^{l}\right\rangle_{c} \tilde{n}_{i}^{l}
$$

and

$$
\tilde{n}_{i}=\sum_{\alpha} \int d \tau n_{i}^{\alpha}(\tau)
$$

$\left\langle v_{i}^{l}\right\rangle_{c}$ denotes the $l$ th cumulant of the disorder distribution. This can be seen by means of the cumulant-generating function which is defined as the logarithm of the left-hand side of eq. (2.15). It follows that

$$
W^{\prime}(\tilde{n})=\ln \left(\int d v p(v) e^{v \tilde{n}}\right)=\sum_{l=1}^{\infty} \frac{1}{l !}\left\langle v^{l}\right\rangle_{c} \tilde{n}^{l} .
$$

Finally, it is assumed that $\left\langle v^{1}\right\rangle_{c}=0$ such that the disorder does not change the filling. If $\left\langle v^{1}\right\rangle_{c} \neq 0$, it can be absorbed in the chemical potential. The effective interaction $W$ that originates from the disorder-average is local in space and translationally invariant. This implies that any single-particle observable $O_{k k^{\prime}} \propto \delta_{k k^{\prime}}$ is diagonal in momentum. In this sense, momentum conservation is restored. 


\subsection{Summary}

In this chapter the Hubbard model and Falicov-Kimball model were introduced. Both models describe two types of interacting electrons on a lattice, the difference between the two models is that both types of electrons are mobile in the Hubbard model, but only one type is for the Falicov-Kimball model.

In section 2.2 disorder was incorporated in both models with a disorder potential that acts on the mobile electrons. The resulting models are the Anderson-Hubbard model and the Anderson-Falicov-Kimball model. Section 2.2.2 dwelled on the physical origin of the disorder potential and the section concluded with the two probability distributions that are used in chapters 6 and 7 to model disorder.

We convinced ourselves that for microscopic systems the solution of the model depends strongly on the disorder realization, therefore the solution of a microscopic system is hardly suitable as an approximation for macroscopic systems. Hence, it was necessary to add disorder-averaging in section 2.2.3 as an additional step in modeling macroscopic disordered systems.

Using the replica trick, it was possible to perform the disorder-average by integrating out the disorder term analytically in favor of an effective translationally invariant interaction between different replicas. The disorder-average restored a pseudo-momentum conservation such that Green functions and other quantities are diagonal in momentum space. This simplifies the methods for disordered interacting lattice problems that are introduced in the following two chapters. 


\section{Conventional Mean-Field Methods for Correlated Lattice Models}

The purpose of this thesis is to develop a method that treats disorder and interactions on equal footing. To this end, we will first introduce the dynamical mean-field theory (DMFT) [26, 27, 28, 29] to approximately handle systems with local interactions. DMFT includes local quantum fluctuations exactly but ignores non-local correlations altogether. This can lead to wrong result when non-local correlations become important, e.g. DMFT predicts the wrong line shape for the metal-insulator transition on the $U T$-plane in $2 \mathrm{~d}$ [85]. Therefore, an important aspect of this thesis is to also introduce and further develop a method that allows to incorporate these non-local correlations.

In spite of its deficiencies DMFT is a useful method. An advantage is that it allows for calculations at very low temperatures even if quantum Monte Carlo (QMC) is used to solve the impurity problem. It does so by mapping the lattice problem to an impurity problem which is numerically relatively cheap to solve. Thus, DMFT allows for quick parameter scans to get a general idea of what to expect for a given system. After such a parameter scan more elaborate methods can be used to study the physics of the system in more detail, taking into account non-local correlations.

In section 3.1 the basic ideas of DMFT are introduced, i. e. the mapping of the lattice problem to an impurity problem and the self-consistency loop. The discussion of the self-consistency loop starts with the original loop of the clean system and is then extended to include disorder [30, 31]. The section on DMFT ends with a brief discussion of the so-called typical-medium-theory (TMT) [86. TMT is designed to distinguish localized states from extended states which enables it to detect Anderson localization. In this thesis, the critical disorder strength from TMT is compared with the dual fermion result to estimate how good the dual fermion approach is in the strong disorder regime.

In section 3.2 the dynamical cluster approximation (DCA) [32, 33] is introduced. It has been used for disordered, interacting and disordered interacting systems. DCA includes short-range correlations by replacing the impurity of DMFT by a cluster embedded in a momentum-dependent dynamical mean-field. Here, it will be shown how to modify the corresponding self-consistency loop to include both disorder and interactions [87, 88].

DCA is numerically much costlier than DMFT. If QMC is used to solve the 

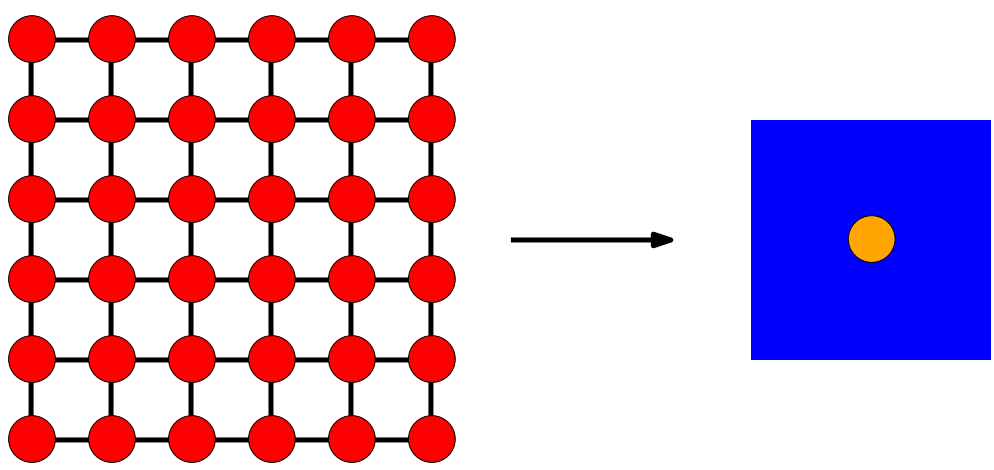

Figure 3.1: In dynamical mean-field theory the lattice of interacting sites is mapped to a single impurity in a non-interacting bath. Instead of solving the lattice problem, an impurity problem is solved self-consistently.

cluster problem, DCA will also suffer from the infamous sign problem away from half-filling. The sign problem is discussed in appendix A. As QMC is the standard cluster solver for interacting quantum systems in DCA, the range of applicability of DCA is limited. Nevertheless, DCA is a well established method that adds back non-local correlations. Comparing the dual fermion approach with DCA will show how good the dual fermion approach is compared to established cluster methods. Thus, it will allow us to estimate how reliable the results will be when new regions of the parameter space are explored.

\subsection{Dynamical Mean-Field Theory}

The basic idea behind a mean-field approach is to treat non-local correlations approximately by considering only one site that is coupled to a mean-field. The mean-field represents the action of the rest of the lattice and in case of fermionic or bosonic models one usually speaks of an impurity coupled to a non-interacting bath. This idea is illustrated in fig. 3.1. The application of this idea to quantum lattice problems with local interactions is called dynamical mean-field theory (DMFT) or sometimes dynamical mean-field approximation, depending on the dimensionality. In infinite dimensions the method is exact and is regarded as a theory, in finite dimensions it is an approximation. In the literature the name dynamical mean-field theory and the abbreviation DMFT are often used independently of the dimension. We will use this convention.

DMFT allows to solve the Hubbard model in infinite dimensions exactly [26] and to investigate the Mott metal-insulator transition [89, 90]. In general, it is a good approximation if the dominant physics is of local nature. If non-local effects start to play a role, the approximation will start to give inaccurate and finally erroneous results. A derivation of the mean-field equation is given in [29]. 
In the Hubbard model the hopping term is the only non-local term. DMFT treats the hopping processes in terms of a mean-field function $G_{0}(\tau)$ that depends on imaginary time $\tau$. The action describing the impurity problem is a functional of $G_{0}$. It reads

$$
\begin{aligned}
S_{e f f}= & -\iint d \tau d \tau^{\prime} \sum_{\sigma} c_{o \sigma}^{\dagger}(\tau) G_{0}\left(\tau-\tau^{\prime}\right)^{-1} c_{o \sigma}\left(\tau^{\prime}\right) \\
& +U \int_{0}^{\beta} d \tau\left(n_{o \uparrow}(\tau)-\frac{1}{2}\right)\left(n_{o \downarrow}(\tau)-\frac{1}{2}\right) .
\end{aligned}
$$

Here, $o$ labels the impurity site. In frequency space $G_{0}$ can be calculated from the impurity self-energy $\Sigma=\Sigma\left[S_{\text {eff }}\right]$ and the local lattice Green function $G_{\text {loc }}=G_{\text {loc }}[\Sigma]$ according to

$$
G_{0}(i \omega)^{-1}=G_{\mathrm{loc}}(i \omega)^{-1}+\Sigma(i \omega) .
$$

The following definition of the single-particle Green function is used:

$$
G_{i j}(\tau)=-\left\langle T_{\tau} c_{i}(\tau) c_{j}^{\dagger}(0)\right\rangle
$$

$T_{\tau}$ is the imaginary-time ordering operator. As usual, $i$ and $j$ label sites.

This set of equations can be solved self-consistently and the procedure will be described in section 3.1.1. We start with the loop for the clean system and then extend it to include disorder. In section 3.1.2 we conclude our introduction of DMFT by presenting the typical-medium-theory (TMT). TMT replaces the average density of states by the typical density of states, which requires access to the real frequency Green function. Otherwise the self-consistency loops of TMT and DMFT are the same.

\subsubsection{Self-Consistent Description of the Local Green Function}

The DMFT equations form a closed set of equations that can be solved self-consistently. The scheme is the following:

0. Make an initial guess for the self-energy, e.g. $\Sigma=0$.

1. Calculate $G_{\text {loc }}(i \omega)=\frac{1}{N} \sum_{k} \frac{1}{i \omega+\mu-\epsilon_{k}-\Sigma(i \omega)}$.

2. Calculate $G_{0}^{-1}(i \omega)=G_{\mathrm{loc}}^{-1}(i \omega)+\Sigma(i \omega)$.

3. Fourier transform $G_{0}(i \omega)$ to obtain $G_{0}(\tau)$. This step is only needed for quantum Monte Carlo methods. 


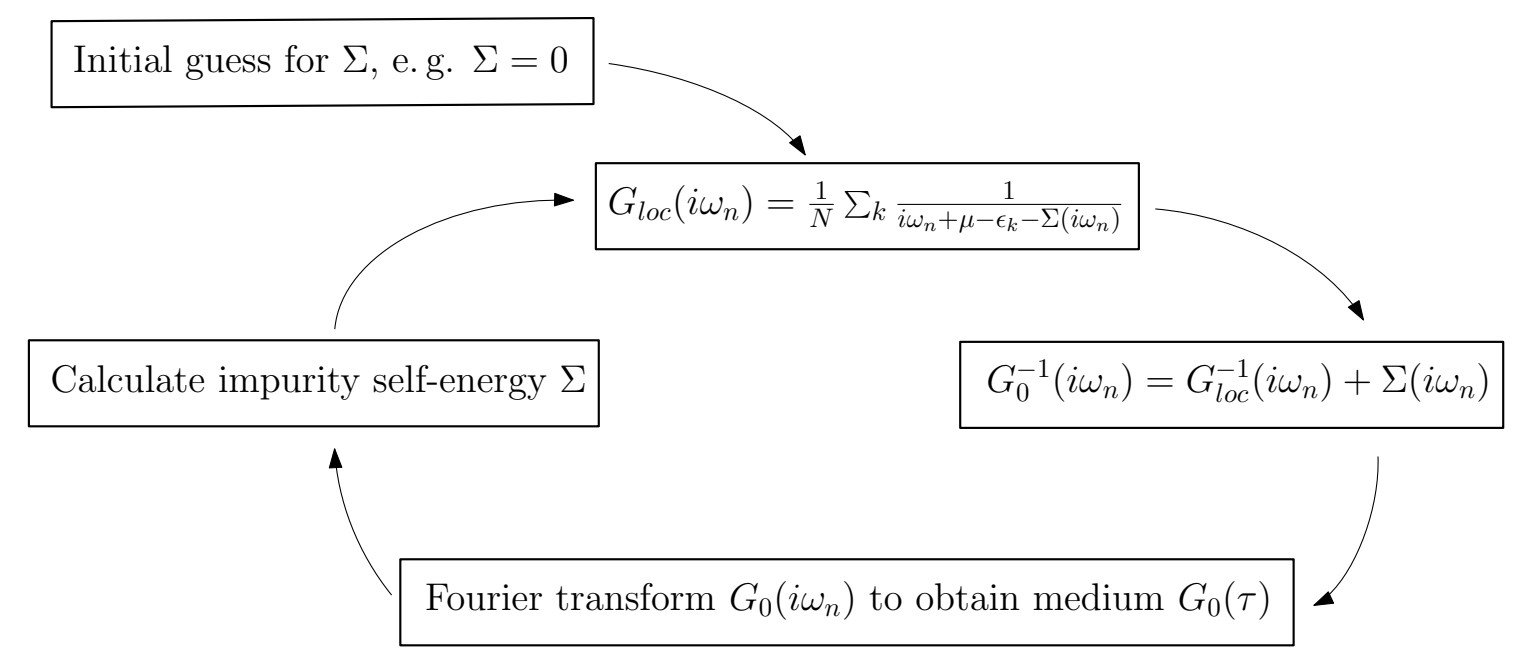

Figure 3.2: DMFT loop for the self-consistent solution of the lattice problem.

4. Solve the impurity problem defined by the medium $G_{0}$ and obtain $\Sigma^{\text {new }}(i \omega)$.

5. Continue with step 1 until convergence for $\Sigma$ is reached.

The superscript "new" refers to the self-energy after the impurity problem. The selfenergy $\Sigma^{\text {new }}$ becomes $\Sigma$ when the loop starts all over again with step 1 . Figure 3.2 shows an illustration of the self-consistency loop. There are different ways to test for convergence, e.g. $\max \left(\left\{\left|\Sigma^{\text {new }}(i \omega)-\Sigma(i \omega)\right|\right\}\right)$ can be used to check the convergence or $\frac{1}{N} \sum_{n=0}^{N-1}\left|\Sigma^{\text {new }}(i \omega)-\Sigma(i \omega)\right|$ where $N$ is the number of frequencies used.

DMFT, as described above, only works for clean interacting systems. In [30, 31 disordered-interacting systems have been treated following the spirit of DMFT. A coherent potential has been introduced to account for the disorder. This procedure is analogous to the Coherent Potential Approximation (CPA) [65, 66] that can be described as DMFT for disordered non-interacting systems. In fact, DMFT for disordered interacting systems reduces to CPA in the non-interacting limit.

The extension of DMFT to include disorder consists of adding disorder-averaging as described in section 2.2.3. The disorder-average is performed on the level of the impurity problem, i. e. one impurity problem is replaced by a collection of impurity problems with different local potentials. The bath Green function for an impurity with a given on-site potential $v_{n}$ is given by

$$
G_{0}^{v_{n}}(i \omega)=\frac{1}{G_{0}^{-1}(i \omega)-v_{n}}
$$

in real space for disorder realization $n . G_{0}$ is the bath Green function for the translationally invariant system. The self-consistency loop of DMFT is altered to the following: 
0. Make an initial guess for the self-energy, e.g. $\Sigma=0$.

1. Calculate $G_{l o c}(i \omega)=\frac{1}{N} \sum_{k} \frac{1}{i \omega+\mu-\epsilon_{k}-\Sigma(i \omega)}$.

2. Calculate $G_{0}^{-1}(i \omega)=G_{l o c}^{-1}(i \omega)+\Sigma(i \omega)$.

3. Solve the disordered impurity problem:

a) Calculate $G_{0}^{v_{n}}=\frac{1}{G_{0}^{-1}-v_{n}}$ for all disorder realizations.

b) Solve the impurity problem defined by the medium $G_{0}^{v_{n}}$ and obtain $g^{v_{n}}(i \omega)$, which is the impurity Green function for the disorder potential $v_{n}$. Do this for all disorder realizations.

c) Calculate $g=\frac{1}{N} \sum_{n=1}^{N} g^{v_{n}}$, where $N$ is the number of disorder realizations.

d) Calculate $\Sigma^{\text {new }}=G_{0}^{-1}-g^{-1}$.

4. Continue with step 1 until convergence for $\Sigma$ is reached.

\subsubsection{Alternative Disorder-Average: The Typical-Medium-Theory}

In the previous subsection the disorder-average was defined as the arithmetic average over disorder configurations. The arithmetic average is most useful in situations where a quantity is symmetrically distributed around its mean value, examples are Gaussian or Lorentzian distributions. For asymmetric distributions like a Poisson distribution or a logarithmic distribution the mean value may not be very meaningful. Instead, the most probable or typical value of a distribution provides a better description of the distribution. The typical value can be obtained from the geometric average.

Weak disorder introduces some variation in the local density of states, which depends on the local potential, but the deviations from the average local density of states (LDOS) will be approximately symmetric. For the case of strong disorder, when the Anderson localization is approached the variation will become stronger and asymmetric as small densities for given $\omega$ become more and more frequent and larger values the exception. When we enter the Anderson insulator the continuous density of states will acquire discrete energy levels for the localized states. These states will be at random energies, thus for a given energy we would expect an LDOS that is 
typically zero. This means that the average value of the LDOS for a given energy does not reflect what one expects to measure. These considerations have been used to develop the typical-medium-theory (TMT) [86]. It can be described as a DMFT where the arithmetic disorder-average is replaced by a geometric disorder-average for the LDOS $\rho$ in the self-consistency according to

$$
\rho_{\text {typ }}(\omega)=\exp \left[\int d v_{n} p\left(v_{n}\right) \ln \rho\left(\omega, v_{n}\right)\right] .
$$

$\rho_{\text {typ }}$ is used in the Hilbert transform for the typical local lattice Green function

$$
G_{\mathrm{typ}}(\omega)=\int d \omega^{\prime} \frac{\rho_{\mathrm{typ}}\left(\omega^{\prime}\right)}{\omega-\omega^{\prime}}
$$

which corresponds to step 1 in the DMFT self-consistency loop.

TMT can distinguish extended states from localized states, therefore it is capable of detecting Anderson localization. The typical LDOS is used as an order parameter. If the typical LDOS vanishes, the system enters the Anderson insulator. TMT is surprisingly good at detecting Anderson localization but the estimate for the critical interaction strength $V_{A}$ can be improved. This is done by extending the single-site TMT approach to a cluster approach, namely cluster-TMT [91] or typical-medium dynamical cluster approximation (TMDCA) [92].

Although TMT is very good at detecting Anderson localization, it is not the method of choice in all cases where disorder is involved. In fact, it has a number of severe drawbacks, i.e. it does not produce correct two-particle quantities and without cluster-extensions it cannot capture true non-local physics. Additionally, it is not capable of producing correct critical exponents [86]. The cluster extensions require the solution of an interacting cluster, thus from a numerical point of view they share the general problems of the dynamical cluster approximation that will be introduced in the next section. 


\subsection{Dynamical Cluster Approximation for Disordered Interacting Systems}

The dynamical cluster approximation (DCA) [32, 33] is an approximation that goes beyond DMFT by including short range correlations within a finite cluster that is used as a reference system. Similar to DMFT the cluster is embedded in a momentum dependent mean-field, which is illustrated in fig. 3.3 . The original lattice coordinate $x=\tilde{x}+X$ is written in terms of the cluster position $\tilde{x}$ and the position $X$ inside the cluster. The lattice momentum $k=K+\tilde{k}$ is written in terms of the cluster momentum $K$ and the momentum $\tilde{k}$ of the superlattice. This is illustrated in fig. 3.4 . Real space and momentum space are related by discrete Fourier transformations

$$
\begin{array}{r}
f(X, \tilde{x})=\frac{N_{c}}{N} \sum_{\tilde{k}} e^{i \tilde{k} \tilde{x}} f(X, \tilde{k}) \\
f(X, \tilde{k})=\sum_{\tilde{x}} e^{-i \tilde{k} \tilde{x}} f(X, \tilde{x}) \\
f(X, \tilde{k})=\frac{1}{N_{c}} \sum_{K} e^{i(K+\tilde{k}) X} f(K, \tilde{k}) \\
f(X, \tilde{k})=\sum_{X} e^{-i(K+\tilde{k}) X} f(X, \tilde{k})
\end{array}
$$

for some function $f . N_{c}$ is the number of cluster sites and $N$ the number of lattice sites. The self-energy $\Sigma(i \omega, k)$ is replaced by the cluster self-energy $\Sigma_{c}(i \omega, K)$ which only depends on the cluster momentum $K$. For $\tilde{k} \neq 0$ we set

$$
\Sigma(i \omega, k)=\Sigma_{c}(i \omega, K)
$$

such that $|k-K|$ is minimal. The cluster self-energy is obtained from the Green function $\mathbf{G}_{c}$ of the isolated cluster via

$$
\mathbf{G}_{c}(i \omega)=\left[(i \omega+\mu) \mathbf{1}-\mathbf{t}_{c}-\Sigma_{c}(i \omega)\right]^{-1}
$$

Bold symbols denote matrices in $K$-space. $\mathbf{t}_{c}$ is the hopping matrix inside the cluster. For the models considered, all quantities are diagonal in spin space.

In a diagrammatic treatment of the full lattice problem, momentum sums have to be performed and momentum conservation is retained at each vertex. This is enforced by the Laue function

$$
\Delta=\sum_{x} e^{i x\left(k_{1}+k_{2}+\cdots-k_{1}^{\prime}-k_{2}^{\prime}-\ldots\right)}=N \delta_{k_{1}+k_{2}+\ldots ; k_{1}^{\prime}+k_{2}^{\prime}+\ldots}
$$



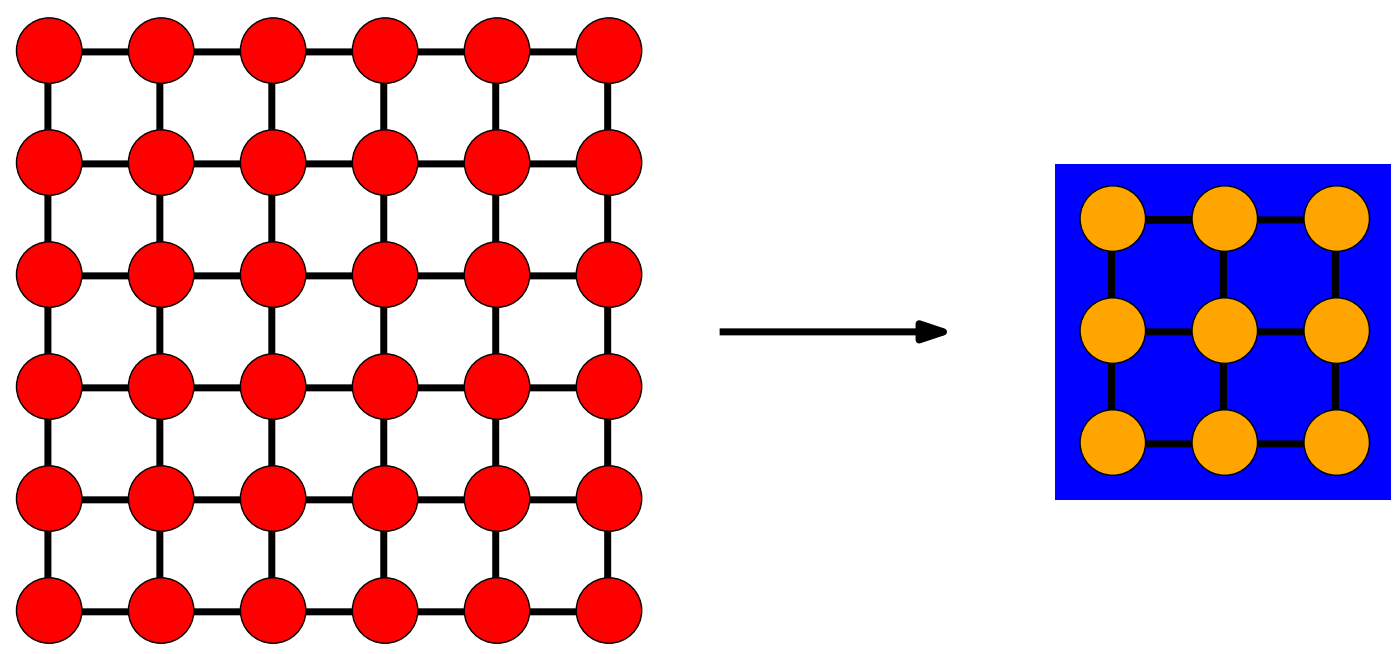

Figure 3.3: The lattice problem is mapped to a cluster embedded in a mean field or bath. In DCA momentum conservation is retained only up to the cluster momenta $K$.
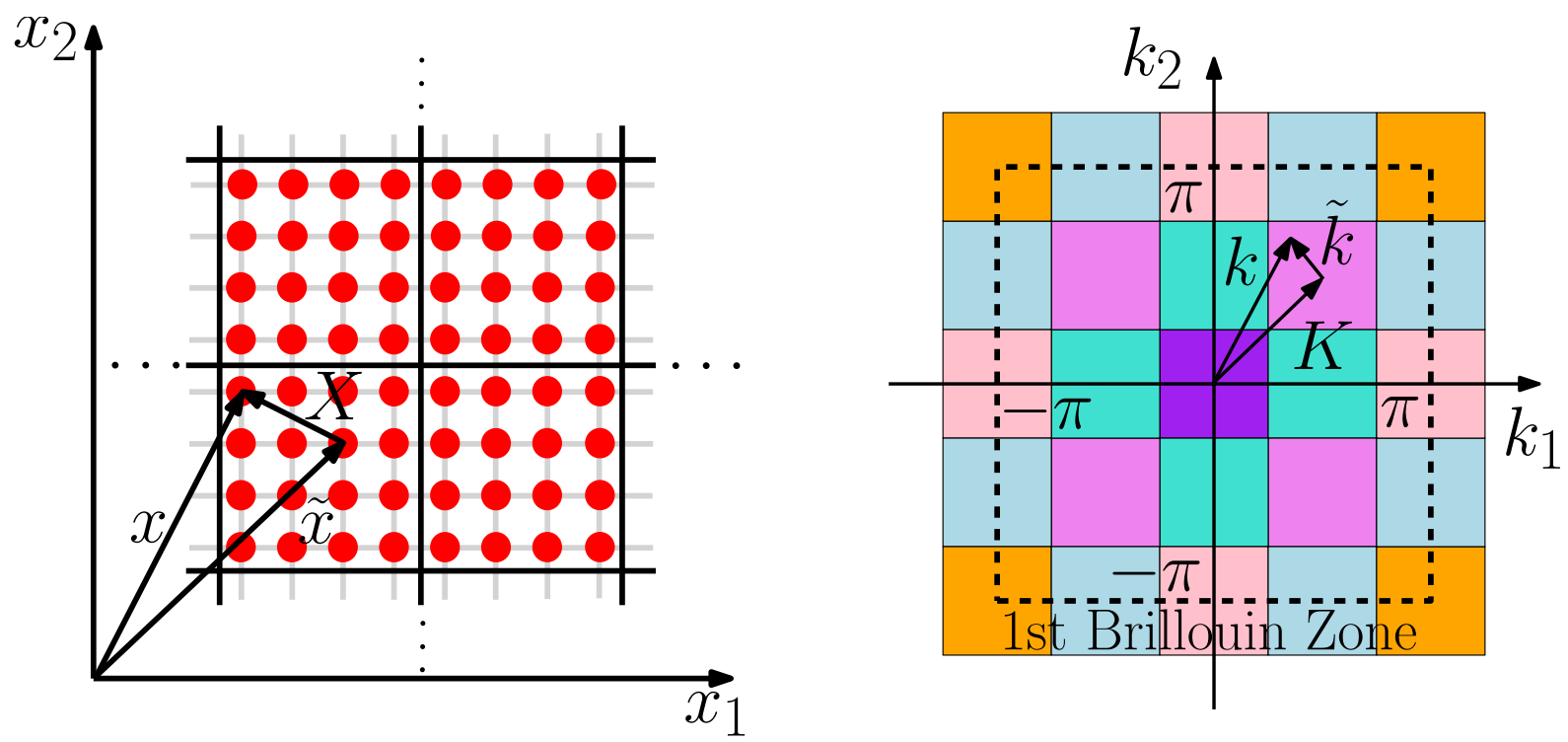

Figure 3.4: The lattice is tiled and a superlattice is introduced. The coordinates of the superlattice are denoted by $\tilde{x}$. The position inside the tiles or clusters are denoted $X$. This leads to a tiling of the Brillouin zone. The coordinates of the superlattice in $k$-space are $K$ and the coordinates within the tiles are $\tilde{k}$. Patches around equivalent $K$-points have the same color. 
In DCA the Laue function is replaced by

$$
\Delta_{D C A}=N_{c} \delta_{K_{1}+K_{2}+\ldots ; K_{1}^{\prime}+K_{2}^{\prime}+\ldots}
$$

This means that the cluster approximation neglects the full momentum conservation. For $N_{c} \rightarrow \infty$ it is systematically restored and for $N_{c}=1$ one obtains DMFT.

\subsubsection{Self-Consistent Description of the Cluster Green Function}

In DCA the coarse-grained lattice Green function $\overline{\mathbf{G}}$ is equated with the cluster Green function $\mathbf{G}_{c}$. In practice, one has to solve a self-consistency loop that relates the cluster and lattice quantities. The self-consistency loop for the DCA goes as follows [84]:

0 . Choose a self-energy $\Sigma_{c}(i \omega, K)$ for the initial calculation. $\Sigma_{c}(i \omega, K) \equiv 0$ is a common choice, but perturbative results can also be used in the hope to reduce the number of self-consistency iterations.

1. Now obtain the coarse-grained lattice Green function

$$
\bar{G}(i \omega, K)=\frac{N_{c}}{N} \sum_{\tilde{k}} \frac{1}{i \omega+\mu-\epsilon_{K+\tilde{k}}-\Sigma_{c}(i \omega, K)} .
$$

2. Calculate the mean-field function

$$
\mathbf{G}_{0}=\left(\overline{\mathbf{G}}^{-1}+\boldsymbol{\Sigma}_{c}\right)^{-1}
$$

3. Obtain the new cluster self-energy $\Sigma_{c}^{\text {new }}$.

Disorder: For the purely disordered system

3.1. Fourier transform $G_{0}(i \omega, K)$ to the $G_{0}(i \omega, i, j)$ matrix representation in real space.

$$
G_{0}(i \omega, i, j)=\frac{1}{N_{c}} \sum_{K} G_{0}(i \omega, K) \exp \left[i K\left(x_{i}-x_{j}\right)\right]
$$

3.2. Calculate

$$
\mathbf{G}_{c}=\left\{\left(\mathbf{G}_{0}^{-1}-\mathbf{V}\right)^{-1}\right\}
$$

where $\{\ldots\}$ indicates the disorder average and $\mathbf{V}$ is the diagonal disorder potential matrix in real space. 
3.3. Transform back to reciprocal space and obtain

$$
\Sigma_{c}^{\text {new }}=G_{0}^{-1}-G_{c}^{-1} .
$$

After the disorder-average $\mathbf{G}_{c}$ is diagonal, such that the scalar inverse can be used in the above.

Interaction: For the purely interacting system solve the cluster problem to obtain $\Sigma_{c}(K)$. For QMC, $G_{0}$ has to be transformed to the right representation in imaginary time and real space:

3.1. Fourier transform $G_{0}(i \omega, K)$ to the imaginary time domain $G_{0}(\tau, K)$.

3.2. Fourier transform $G_{0}(\tau, K)$ to the real space $G_{0}(\tau, i)$.

3.3. Solve the cluster problem and obtain $\Sigma_{c}^{\text {new }}(i \omega, K)$.

4. Start from step 1, again, setting $\Sigma_{c}=\Sigma_{c}^{\text {new }}$.

Now, we have to merge these two algorithms that differ in step 3 . To this end, let us understand what the main difference is. For the purely interacting problem all quantities are diagonal in momentum space. For the disordered system, however, $V$ breaks the translational invariance of the cluster and therefore the Green function $G_{c}^{\text {conf }}$ for a single disorder configuration is no longer diagonal in momentum space. It will only be diagonal after the disorder average $\{\ldots\}$ has been performed. The step we have to modify is 3.2 . for the disordered system, where the term in $\{\ldots\}$ has to be replaced by the solution of the interacting disordered cluster. The effect of disorder is to change $G_{0}$ for the individual disorder configurations:

$$
\left(\mathbf{G}_{0}^{\text {conf }}\right)^{-1}=\mathbf{G}_{0}^{-1}-\mathbf{V} .
$$

We are now dealing with a $G_{0}^{\text {conf }}$ that is neither diagonal in real space nor in momentum space.

QMC (c.f. chapter 5) is used in this work to solve the disordered interacting cluster problem. In the following we present in detail, how $G_{0}^{\text {conf }}(\tau, i, j)$ is obtained. Additionally, $G_{0}^{\operatorname{conf}}\left(i \omega, K, K^{\prime}\right)$ is needed to calculate $G_{c}^{\text {conf }}\left(i \omega, K, K^{\prime}\right)$ as we will see later.

Starting from $G_{0}(i \omega, K)$ we do the following steps:

1. We Fourier transform from reciprocal to real space

$$
G_{0}(i \omega, i, j)=\frac{1}{N_{c}} \sum_{K} G_{0}(i \omega, K) \exp \left[i K\left(x_{i}-x_{j}\right)\right] .
$$


2. Then $\mathbf{G}_{0}^{\operatorname{conf}}(i \omega, i, j)=\left(\mathbf{G}_{0}^{-1}-\mathbf{V}\right)^{-1}(i \omega, i, j)$ is evaluated.

3. Next, a Fourier transform leads back to the reciprocal space Green function

$$
G_{0}^{\mathrm{conf}}\left(i \omega, K, K^{\prime}\right)=\frac{1}{N_{c}} \sum_{i j} G_{0}^{\mathrm{conf}}(i \omega, i, j) \exp \left[-i\left(K x_{i}-K^{\prime} x_{j}\right)\right] .
$$

The factor $\frac{1}{N_{c}}$ is needed to adjust the dimensionality in terms of $N_{c}$.

4. Then Fourier transforming $G_{0}^{\text {conf }}\left(i \omega, K, K^{\prime}\right)$ to the imaginary time domain leads to $G_{0}^{\text {conf }}\left(\tau, K, K^{\prime}\right)$.

5. Finally, a Fourier transformation is performed to get back to real space. Only the diagonal entries are needed in

$$
G_{0}^{\text {conf }}(\tau, i, j)=\frac{1}{N_{c}^{2}} \sum_{K K^{\prime}} G_{0}^{\text {conf }}\left(\tau, K, K^{\prime}\right) \exp \left[i\left(K x_{i}-K^{\prime} x_{j}\right)\right]
$$

With $G_{0}^{\text {conf }}(\tau, i, j)$ one can calculate $\Sigma_{c}$ using QMC to solve the cluster problem. At first glance it may seem we perform some unnecessary Fourier transforms. However, for the imaginary time Fourier transform we need high-frequency conditioning. Highfrequency conditioning means that we treat the high-frequency behavior of the Green function analytically. This is particularly simple in $(i \omega, K)$-space where the Green function decays as $1 /(i \omega)[93,94]$.

\subsubsection{Changes to the Impurity Solver for Clean Systems}

We start from a QMC code for a translationally invariant cluster, which is introduced in chapter 5. Therefore, quantities only depend on the difference between two sites but not the sites themselves. This is different for disordered systems, where we need the full real space matrix $\mathbf{G}_{0}$ for the updates.

Furthermore, also the measurement of the Green function is changed if we want to measure in $K$ and $i \omega$ space. For the measurement we use the equation (c.f. eq. $(5.25)$

$G(i \omega, K)=G_{0}(i \omega, K)-\frac{1}{\beta} G_{0}^{2}(i \omega, K)\left\langle\sum_{p q}^{k} \exp \left[i K\left(n_{p}-n_{q}\right)\right] \exp \left[i \omega\left(\tau_{p}-\tau_{q}\right)\right]\left(M_{k}\right)_{p q}\right\rangle_{\mathrm{QMC}}$.

$M$ is the configuration matrix of the Monte Carlo simulation and $p$ and $q$ are matrix indices. $\langle\ldots\rangle_{\mathrm{QMC}}$ denotes a Monte Carlo average. We have to adopt this formula 
to account for the off-diagonal parts of $G_{0}^{\text {conf }}$ which replaces $G_{0}$ for the disordered system. Performing the Fourier transform in two space variables leads to

$$
\begin{aligned}
G^{\operatorname{conf}}\left(i \omega, K, K^{\prime}\right)=G_{0}^{\operatorname{conf}}(i \omega & \left., K, K^{\prime}\right)-\frac{1}{\beta N_{c}^{2}} \sum_{K_{1} K_{2}} G_{0}^{\operatorname{conf}}\left(i \omega, K, K_{1}\right) G_{0}^{\operatorname{conf}}\left(i \omega, K_{2}, K^{\prime}\right) \times \\
\times & \left\langle\sum_{p q}^{k} \exp \left[i\left(K_{1} n_{p}-K_{2} n_{q}\right)\right] \exp \left[i \omega\left(\tau_{p}-\tau_{q}\right)\right]\left(M_{k}\right)_{p q}\right\rangle_{\mathrm{QMC}} .
\end{aligned}
$$

At the end, $\mathbf{G}^{\text {conf }}$ will be disorder averaged and only the terms diagonal in $K$ will survive.

Alternatively, one can measure the cluster Green function in $r$ and $\tau$ space. One has to measure only the diagonal elements in $r$ space, because all other elements will not survive the disorder-averaging. This is because the Green function is diagonal in $k$-space after the disorder-average as shown in section 2.2 .3 .

\subsection{Summary}

In this chapter, we introduced two mean-field methods for the solution of interacting electrons on a disordered lattice. These methods have been developed prior to the dual fermion approach. Both rely on the mapping to an impurity or cluster problem that has to be solved self-consistently.

First, in section 3.1 DMFT was introduced. It is exact in infinite dimensions, but ignores non-local correlations altogether if applied to finite dimensional systems. This neglect of non-local correlations is what we want to overcome. The section about DMFT was closed with an introduction of TMT, which is a modification of DMFT that is able to detect Anderson localization.

Second, in section 3.2 DCA was discussed, a method developed to improve DMFT by including short-range correlations. DCA is a powerful method for interacting systems, but it is limited to relatively small system sizes, especially in 3d. Generally Quantum Monte Carlo is used to solve the cluster problem, which means that DCA indirectly suffers from the sign problem away from half-filling. We use DCA as a benchmark for the dual fermion approach presented in the next chapter. 


\section{The Dual Fermion Approach for Disordered Interacting Systems}

The dynamical mean-field theory (DMFT) presented in section 3.1 neglects nonlocal correlations. Thus, depending on the system under investigation, DMFT can be quite a crude approximation giving qualitatively wrong results, for example in the vicinity of antiferromagnetism or charge-density waves.

One way to add back non-local correlations is by means of cluster methods, e. g. the dynamical cluster approximation (DCA) [32, 33, cellular DMFT (cDMFT) [34] or large scale quantum Monte Carlo (QMC) [42, 43] simulations. If DCA and cDMFT use QMC to solve the cluster problem, all three methods will in general suffer from the sign problem. Therefore, these three methods are often limited to rather short-ranged correlations as the cluster sizes are usually quite small because an increasing cluster size worsens the sign problem. This is especially problematic in more than one dimension.

In one dimension it is possible to overcome the sign problem [44]. For translationally invariant single-orbital Hubbard-like systems the sign problem can be overcome at half-filling as well. However, in the case of disorder there will generally be disorder realizations that are away from half-filling. Thus, in more than one dimension the sign problem is typically present.

Other cluster methods in the context of disorder are the traveling cluster approximation [95, 96], the molecular coherent potential approximation [97, 98, 99] and the cluster coherent potential approximation [100, 101, 102, 103, 104].

Another way to add back non-local correlations is to use a perturbative approach. The calculations involved are usually less costly and they do not suffer from the sign problem if a corresponding reference system can be chosen. However, ordinary perturbation theory suffers from the problem that one has to find a small expansion parameter, which is not an easy task for the Hubbard model.

A way out is to introduce new degrees of freedom that allow to do a perturbative expansion in the non-local correlations only. Around the DMFT result for small values of $U$ non-local correlations will be small. On the other hand, for strong interactions, i. e. around the atomic limit, non-local correlations will not be significant either. Therefore, an expansion in terms of the non-local contributions is desirable. 
This can be achieved by means of a Hubbard-Stratonovich transformation ${ }^{1}$ which is shown in [105, 36, 37] for an interacting system. The Hubbard-Stratonovich transformation introduces new degrees of freedom which are referred to as dual fermions. We will refer to the original degrees of freedom as real fermions. Terletska et al. [40] applied the approach to a disordered system. Here, we want to apply it to a disordered interacting problem. For the derivation of the formalism we extend the derivation of Terletska et al. [40]. We published the application to the Anderson-Falicov-Kimball model in [106]. The derivation for this model is presented in appendix C. Here, we will focus on the derivation for the Anderson-Hubbard model.

By construction, the dual fermion approach is not a mean-field method, i. e. there is no a priori need to do some coarse graining as for DMFT and DCA. In practice, the dual fermion approach uses a self-consistency loop. The self-consistency condition is formulated in terms of local quantities and calculating those quantities essentially requires coarse-graining. Therefore, dual fermions are likely to retain a mean-field character.

One has to note that other approaches that extend DMFT by means of a perturbative expansion do exist, going back to [35]. One such approach is the dynamical vertex approximation (DГA) [39, 38, which is the main competitor of dual fermions. DГA uses the local irreducible vertex function to create an approximation for higher order reducible vertex functions.

\subsection{Derivation of the Formalism}

The derivation of the formalism will be done using the path integral formalism ${ }^{2}$ for the partition function. We will apply the formalism to quenched disorder. Unlike for the clean system or systems with annealed disorder, one has to deal with $\{\ln Z\} \neq$ $\ln \{Z\}$, where $\{\ldots\}$ as usual denotes a disorder-average. Instead of introducing the dual degrees of freedom for $Z$, as done in [105, 36, 37], we will do so for $\{\ln Z\}$. The action of the lattice model will be rewritten in terms of an impurity action. Then the replica trick is used to express $\{\ln Z\}$ in terms of powers of $\{Z\}$ and to integrate out the disorder. A Hubbard-Stratonovich transformation is used to decouple the non-local real degrees of freedom and introduce dual degrees of freedom. As a result, the real degrees of freedom can be integrated out. This is done by defining the dual potential and deriving an expression for it.

As has been discussed in section 2.2.3, the disorder-average is applied to observ-

\footnotetext{
${ }^{1}$ The transformation used is actually more general than a Hubbard-Stratonovich transformation, but the name has established itself in the field of dual fermions and thus it is used in this thesis.

${ }^{2} \mathrm{An}$ introduction to path integrals can be found in [107].
} 
ables, e.g. for the Green function this implies

$$
\left\{G_{k}\right\}=-\left.\frac{\delta}{\delta \eta}\{\ln Z\}\right|_{\eta=0},
$$

where $\eta$ is a source field and $Z$ is the partition function. The partition function is defined as

$$
Z=\int \prod_{\omega k} d \bar{c}_{\omega k} d c_{\omega k} e^{-S}
$$

in the path integral formalism. $c$ and $\bar{c}$ are Grassmann numbers. To shorten the notation, $\omega \equiv i \omega_{n}$ is used to label Matsubara frequencies and $k$ is a momentum label.

The action for a given disorder configuration is

$$
S^{v}[\bar{c}, c]=-\sum_{\omega k \sigma} \bar{c}_{\omega k \sigma}\left(\omega+\mu-\epsilon_{k}\right) c_{\omega k \sigma}+\sum_{i} S_{i}^{v}\left[\bar{c}_{i}, c_{i}\right]
$$

with the local part of the action

$$
S_{i}^{v}\left[\bar{c}_{i}, c_{i}\right]=-\int_{0}^{\beta} d \tau v_{i} n_{i}(\tau)+\int_{0}^{\beta} d \tau U n_{\uparrow i}(\tau) n_{\downarrow i}(\tau)
$$

at site $i$. In terms of an impurity action

$$
S_{i}^{v, \operatorname{imp}}\left[\bar{c}_{i}, c_{i}\right]=-\sum_{\omega, \sigma} \bar{c}_{\omega \sigma}\left[(\omega+\mu)-\Delta_{\omega}\right] c_{\omega \sigma}+S_{i}^{v}\left[\bar{c}_{i}, c_{i}\right]
$$

the action $S^{v}$ is rewritten as

$$
S^{v}[\bar{c}, c]=\sum_{i} S_{i}^{v, \text { imp }}\left[\bar{c}_{i}, c_{i}\right]-\sum_{\omega k \sigma} \bar{c}_{\omega k \sigma}\left(\Delta_{\omega}-\epsilon_{k}\right) c_{\omega k \sigma}
$$

$\Delta_{\omega}$ is a hybridization function that is not yet specified. In section 4.4 we will show how it can be determined self-consistently.

As it is inconvenient to deal with $\{\ln Z\}$, we use the replica trick [81, 82] to integrate out the disorder as shown in section 2.2.3. In this approach, we use the replica trick to derive the formalism and to obtain diagrams. We will not use it to do calculations and extrapolate $m \rightarrow 0$ for our data. This extrapolation is done only for the diagrams. Therefore, we are not concerned about replica symmetry breaking [83]. 
The replica trick leads to

$$
\begin{aligned}
\{\ln Z\} & =\int \prod_{i} d v_{i} p\left(v_{i}\right) \ln Z^{v} \\
& =\lim _{m \rightarrow 0} \frac{1}{m}\left(\int \prod_{i} d v_{i} p\left(v_{i}\right) \int D\left[\bar{c}_{i}, c_{i}\right] e^{-S_{R}^{v}}-1\right),
\end{aligned}
$$

where

$$
\begin{aligned}
S_{R}^{v}= & -\sum_{\alpha} \sum_{\omega k \sigma} \bar{c}_{\omega k \sigma}^{\alpha}\left(\omega+\mu-\epsilon_{k}\right) c_{\omega k \sigma}^{\alpha}-\sum_{i, \alpha} \int_{0}^{\beta} d \tau v_{i} n_{i}^{\alpha}(\tau)+\sum_{i, \alpha} \int_{0}^{\beta} d \tau U n_{\uparrow i}^{\alpha}(\tau) n_{\downarrow i}^{\alpha}(\tau) \\
= & -\sum_{\alpha} \sum_{\omega i \sigma} \bar{c}_{\omega i \sigma}^{\alpha}\left(\omega+\mu-\Delta_{\omega}\right) c_{\omega i \sigma}^{\alpha}-\sum_{\alpha} \sum_{\omega k \sigma} \bar{c}_{\omega k \sigma}^{\alpha}\left(\Delta_{\omega}-\epsilon_{k}\right) c_{\omega k \sigma}^{\alpha} \\
& -\sum_{i, \alpha} \int_{0}^{\beta} d \tau v_{i} n_{i}^{\alpha}(\tau)+\sum_{i, \alpha} \int_{0}^{\beta} d \tau U n_{\uparrow i}^{\alpha}(\tau) n_{\downarrow i}^{\alpha}(\tau) \\
= & \sum_{i} S_{i, R}^{v, i m p}-\sum_{\alpha} \sum_{\omega k \sigma} \bar{c}_{\omega k \sigma}^{\alpha}\left(\Delta_{\omega}-\epsilon_{k}\right) c_{\omega k \sigma}^{\alpha}
\end{aligned}
$$

with

$S_{i, R}^{v, \text { imp }}=-\sum_{\alpha} \sum_{\omega \sigma} \bar{c}_{\omega i \sigma}^{\alpha}\left(\omega+\mu-\Delta_{\omega}\right) c_{\omega i \sigma}^{\alpha}-\sum_{\alpha} \int_{0}^{\beta} d \tau v_{i} n_{i}^{\alpha}(\tau)+\sum_{\alpha} \int_{0}^{\beta} d \tau U n_{\uparrow i}^{\alpha}(\tau) n_{\downarrow i}^{\alpha}(\tau)$

and $D\left[\bar{c}_{i}, c_{i}\right]=\prod_{\omega \alpha} d \bar{c}_{\omega i}^{\alpha} d c_{\omega i}^{\alpha}$. Greek letters $\alpha, \beta \ldots \epsilon$ that appear as indices here and in the following are replica labels. Be aware not to confuse the replica label $\beta$ and $\epsilon$ with the inverse temperature $\beta$ and the dispersion relation $\epsilon$. In the above, the replica limit introduced $\lim _{m \rightarrow 0} \frac{-1}{m}$. This terms is a constant with respect to derivatives of the partition function and because such derivatives are performed before the replica limit there will be no problem with $\frac{1}{0}$.

It is now possible to integrate out the disorder as was done in section 2.2.3. This leads to the action

$$
S_{R}=\sum_{i} S_{\mathrm{imp}}^{i}-\sum_{\alpha} \sum_{\omega k \sigma} \bar{c}_{\omega k \sigma}^{\alpha}\left(\Delta_{\omega}-\epsilon_{k}\right) c_{\omega k \sigma}^{\alpha}
$$

with

$$
S_{\mathrm{imp}}^{i}=-\sum_{\alpha} \sum_{\omega \sigma} \bar{c}_{\omega i \sigma}^{\alpha}\left(\omega+\mu-\Delta_{\omega}\right) c_{\omega i \sigma}^{\alpha}-W\left(\tilde{n}_{i}\right)+\sum_{\alpha} \int_{0}^{\beta} d \tau U n_{\uparrow i}^{\alpha}(\tau) n_{\downarrow i}^{\alpha}(\tau) .
$$


The effective disorder interaction is given by

$$
W\left(\tilde{n}_{i}\right)=\sum_{l=2}^{\infty} \frac{1}{l !}\left\langle v_{i}^{l}\right\rangle_{c} \tilde{n}_{i}^{l}
$$

and

$$
\tilde{n}_{i}=\sum_{\alpha} \int d \tau n_{i}^{\alpha}(\tau)
$$

$\left\langle v_{i}^{l}\right\rangle_{c}$ is the $l$ th cumulant of the disorder distribution. This was derived in section 2.2 .3 .

In the following, we will omit the spin index for better readability. It is easy to reintroduce the spin index if needed. All configurations which conserve the spin are possible, e. g., $\left\langle c_{\uparrow} c_{\downarrow} \bar{c}_{\uparrow} \bar{c}_{\downarrow}\right\rangle$ is possible, $\left\langle c_{\uparrow} c_{\downarrow} \bar{c}_{\uparrow} \bar{c}_{\uparrow}\right\rangle$ is not.

The next step is to perform a Hubbard-Stratonovich transformation

$$
e^{\bar{c}_{\omega k}^{\alpha} A_{\omega k}^{2} c_{\omega k}}=\frac{A_{\omega k}^{2}}{\lambda_{\omega}^{2}} \int D[\bar{f}, f] e^{-\lambda_{\omega}\left(\bar{c}_{\omega k}^{\alpha} f_{\omega k}^{\alpha}+\bar{f}_{\omega k}^{\alpha} c_{\omega k}^{\alpha}\right)-\frac{\lambda_{\omega}^{2}}{A_{\omega k}^{2}} \bar{f}_{\omega k}^{\alpha} f_{\omega k}^{\alpha}},
$$

which introduces new $f$ degrees of freedom. These are Grassmann numbers ${ }^{3}$, i. e. they follow fermionic commutation relations. Hence the formalism is called dual fermions. The measure above is defined as $D[\bar{f}, f]=\prod_{\omega k \alpha} d \bar{f}_{\omega k}^{\alpha} d f_{\omega k}^{\alpha}$. Comparing eqs. 4.10) and (4.14), one finds that

$$
A_{\omega k}^{2}=\Delta_{\omega}-\epsilon_{k}
$$

but $\lambda_{\omega}$ remains arbitrary. It follows that

$$
\begin{aligned}
\{\ln Z\}= & \lim _{m \rightarrow 0} \frac{1}{m}\left[\left(\prod_{\omega k} \frac{\Delta_{\omega}-\epsilon_{k}}{\lambda_{\omega}^{2}}\right)^{m} \int D[\bar{f}, f] e^{-\sum_{\omega k \alpha} \lambda_{\omega}^{2} \bar{f}_{\omega k}^{\alpha}\left(\Delta_{\omega}-\epsilon_{k}\right)^{-1} f_{\omega k}^{\alpha} \times}\right. \\
& \left.\times \int D \bar{c} D c e^{-\sum_{i} S_{\text {site }}^{i}}-1\right]
\end{aligned}
$$

with

$$
S_{\mathrm{site}}^{i}=S_{\mathrm{imp}}^{i}+\sum_{\omega \alpha} \lambda_{\omega}\left(\bar{c}_{\omega i}^{\alpha} f_{\omega i}^{\alpha}+\bar{f}_{\omega i}^{\alpha} c_{\omega i}^{\alpha}\right)
$$

$S_{\text {site }}$ does not contain any non-local terms. In fact, all non-local correlations have been removed from the $c$-degrees of freedom and have been transfered to the $f$ degrees of freedom by means of the Hubbard-Stratonovich transformation. Therefore, the $c$-degrees of freedom can be integrated out.

In the following, the dual potential will be defined and an expression for it will be derived. It will turn out that the dual potential is related to two-particle vertex

\footnotetext{
${ }^{3}$ To shorten the notation and increase the readability, we sometimes refer to the $c / f$ Grassmann numbers as $c / f$ operators.
} 
functions. In appendix $\mathrm{D}$ two-particle Green functions and vertex functions are discussed in more detail.

The dual potential is defined via

$$
\int D[\bar{c}, c] e^{-S_{\text {site }}\left[\bar{c}^{\alpha}, c^{\alpha} ; \bar{f}^{\alpha}, f^{\alpha}\right]}=Z_{\mathrm{imp}} e^{-\sum_{\omega \alpha} \lambda_{\omega}^{2} g(\omega) \bar{f}_{\omega}^{\alpha} f_{\omega}^{\alpha}-V_{d}\left[\bar{f}^{\alpha}, f^{\beta}\right]}
$$

where $g$ is the disorder-averaged impurity Green function. Note that expectation values with respect to $S_{\text {site }}$ include the disorder-average. The impurity partition function $Z_{\text {imp }}$ is defined as

$$
Z_{\mathrm{imp}}=\int D[\bar{c}, c] e^{-S_{\mathrm{imp}}}
$$

Equation (4.18) is expanded on both sites and compared order by order.

We start with expanding the left hand side of eq. 4.18 and we obtain

$$
\int D[\bar{c}, c] e^{-S_{\text {site }}}=\int D[\bar{c}, c] e^{-S_{\mathrm{imp}}} \sum_{n=0}^{\infty} \frac{(-1)^{n}}{n !}\left(\sum_{\omega \alpha} \lambda_{\omega}\left(\bar{c}_{\omega}^{\alpha} f_{\omega}^{\alpha}+\bar{f}_{\omega}^{\alpha} c_{\omega}^{\alpha}\right)\right)^{n} .
$$

Only even orders of $n$ survive the integration, because expectation values like $\langle c\rangle$, $\langle\bar{c} c c\rangle, \ldots$ with an odd number of operators vanish. Also, for even orders, only contributions with the same number of $\bar{c}$ and $c$ have to be considered because expectation values like $\langle c c\rangle$ vanish for the models in this thesis.

In lowest order the expansion yields

$$
n=0: \quad \int D[\bar{c}, c] e^{-S_{\mathrm{imp}}}=Z_{\mathrm{imp}}
$$

For the second order the contribution is

$$
\begin{aligned}
n=2: & \int D[\bar{c}, c] e^{-S_{\mathrm{imp}}} \frac{1}{2} \sum_{\alpha \beta \omega \omega^{\prime}} \lambda_{\omega} \lambda_{\omega^{\prime}}\left(\bar{c}_{\omega}^{\alpha} f_{\omega}^{\alpha}+\bar{f}_{\omega}^{\alpha} c_{\omega}^{\alpha}\right)\left(\bar{c}_{\omega^{\prime}}^{\beta} f_{\omega^{\prime}}^{\beta}+\bar{f}_{\omega^{\prime}}^{\beta} c_{\omega^{\prime}}^{\beta}\right) \\
= & \int D[\bar{c}, c] e^{-S_{\mathrm{imp}}} \frac{1}{2} \sum_{\alpha \beta \omega \omega^{\prime}} \lambda_{\omega} \lambda_{\omega^{\prime}}\left(\bar{c}_{\omega}^{\alpha} c_{\omega^{\prime}}^{\beta} f_{\omega}^{\alpha} \bar{f}_{\omega^{\prime}}^{\beta}+c_{\omega}^{\alpha} \bar{c}_{\omega^{\prime}}^{\beta} \bar{f}_{\omega}^{\alpha} f_{\omega^{\prime}}^{\beta}\right) \\
= & \int D[\bar{c}, c] e^{-S_{\mathrm{imp}}} \frac{1}{2} \sum_{\alpha \beta \omega \omega^{\prime}} \lambda_{\omega} \lambda_{\omega^{\prime}}\left(\bar{c}_{\omega}^{\alpha} c_{\omega^{\prime}}^{\beta} f_{\omega}^{\alpha} \bar{f}_{\omega^{\prime}}^{\beta}+c_{\omega}^{\alpha} \bar{c}_{\omega^{\prime}}^{\beta} \bar{f}_{\omega}^{\alpha} f_{\omega^{\prime}}^{\beta}\right) \delta_{\omega \omega^{\prime}} \delta_{\alpha \beta} \\
= & \int D[\bar{c}, c] e^{-S_{\text {imp }}} \frac{1}{2} \sum_{\alpha \omega} \lambda_{\omega}^{2}\left(\bar{c}_{\omega}^{\alpha} c_{\omega}^{\alpha} f_{\omega}^{\alpha} \bar{f}_{\omega}^{\alpha}+c_{\omega}^{\alpha} \bar{c}_{\omega}^{\alpha} \bar{f}_{\omega}^{\alpha} f_{\omega}^{\alpha}\right) \\
= & \int D[\bar{c}, c] e^{-S_{\mathrm{imp}}} \frac{1}{2} \sum_{\alpha \omega} \lambda_{\omega}^{2} 2 \bar{c}_{\omega}^{\alpha} c_{\omega}^{\alpha} f_{\omega}^{\alpha} \bar{f}_{\omega}^{\alpha} \\
= & -Z_{\operatorname{imp}} \sum_{\omega \alpha} \lambda_{\omega}^{2} g_{\omega}^{\alpha} \bar{f}_{\omega}^{\alpha} f_{\omega}^{\alpha} .
\end{aligned}
$$


Equation 4.22c follows from eq. 4.22b by noting that there is no travel of electrons between different replicas and time translational invariance, i. e. $\left\langle c_{\omega}^{\alpha} \bar{c}_{\omega^{\prime}}^{\beta}\right\rangle \propto \delta_{\alpha \beta} \delta_{\omega \omega^{\prime}}$. Finally, the definition of the Green function eq. (3.3) leads to eq. 4.22f).

In fourth order the expansion yields

$$
\begin{aligned}
& n=4: \frac{1}{24} \int D[\bar{c}, c] e^{-S_{\mathrm{imp}}} \sum_{\substack{\alpha \beta \gamma \delta \\
\omega_{1} \omega_{2} \omega_{3} \omega_{4}}} \lambda_{\omega_{1}} \lambda_{\omega_{2}} \lambda_{\omega_{3}} \lambda_{\omega_{4}}\left(\bar{c}_{\omega_{1}}^{\alpha} f_{\omega_{1}}^{\alpha}+\bar{f}_{\omega_{1}}^{\alpha} c_{\omega_{1}}^{\alpha}\right) \\
& \times\left(\bar{c}_{\omega_{2}}^{\beta} f_{\omega_{2}}^{\beta}+\bar{f}_{\omega_{2}}^{\beta} c_{\omega_{2}}^{\beta}\right)\left(\bar{c}_{\omega_{3}}^{\gamma} f_{\omega_{3}}^{\gamma}+\bar{f}_{\omega_{3}}^{\gamma} c_{\omega_{3}}^{\gamma}\right)\left(\bar{c}_{\omega_{4}}^{\delta} f_{\omega_{4}}^{\delta}+\bar{f}_{\omega_{4}}^{\delta} c_{\omega_{4}}^{\delta}\right) \\
& =\frac{1}{24} \int D[\bar{c}, c] e^{-S_{\mathrm{imp}}} \sum_{\substack{\alpha \beta \gamma \delta \\
\omega_{1} \omega_{2} \omega_{3} \omega_{4}}} \lambda_{\omega_{1}} \lambda_{\omega_{2}} \lambda_{\omega_{3}} \lambda_{\omega_{4}} \\
& \times\left(\bar{c}_{\omega_{1}}^{\alpha} c_{\omega_{2}}^{\beta} \bar{c}_{\omega_{3}}^{\gamma} c_{\omega_{4}}^{\delta} f_{\omega_{1}}^{\alpha} \bar{f}_{\omega_{2}}^{\beta} f_{\omega_{3}}^{\gamma} \bar{f}_{\omega_{4}}^{\delta}+\bar{c}_{\omega_{1}}^{\alpha} \bar{c}_{\omega_{2}}^{\beta} c_{\omega_{3}}^{\gamma} c_{\omega_{4}}^{\delta} f_{\omega_{1}}^{\alpha} f_{\omega_{2}}^{\beta} \bar{f}_{\omega_{3}}^{\gamma} \bar{f}_{\omega_{4}}^{\delta}\right. \\
& +\bar{c}_{\omega_{1}}^{\alpha} c_{\omega_{2}}^{\beta} c_{\omega_{3}}^{\gamma} \bar{c}_{\omega_{4}}^{\delta} f_{\omega_{1}}^{\alpha} \bar{f}_{\omega_{2}}^{\beta} \bar{f}_{\omega_{3}}^{\gamma} f_{\omega_{4}}^{\delta}+c_{\omega_{1}}^{\alpha} \bar{c}_{\omega_{2}}^{\beta} \bar{c}_{\omega_{3}}^{\gamma} c_{\omega_{4}}^{\delta} \bar{f}_{\omega_{1}}^{\alpha} f_{\omega_{2}}^{\beta} f_{\omega_{3}}^{\gamma} \bar{f}_{\omega_{4}}^{\delta} \\
& \left.+c_{\omega_{1}}^{\alpha} \bar{c}_{\omega_{2}}^{\beta} c_{\omega_{3}}^{\gamma} \bar{c}_{\omega_{4}}^{\delta} \bar{f}_{\omega_{1}}^{\alpha} f_{\omega_{2}}^{\beta} \bar{f}_{\omega_{3}}^{\gamma} f_{\omega_{4}}^{\delta}+c_{\omega_{1}}^{\alpha} c_{\omega_{2}}^{\beta} \bar{c}_{\omega_{3}}^{\gamma} \bar{c}_{\omega_{4}}^{\delta} \bar{f}_{\omega_{1}}^{\alpha} \bar{f}_{\omega_{2}}^{\beta} f_{\omega_{3}}^{\gamma} f_{\omega_{4}}^{\delta}\right) \\
& =\frac{1}{4} \int D[\bar{c}, c] e^{-S_{\mathrm{imp}}} \sum_{\substack{\alpha \beta \gamma \delta \\
\omega_{1} \omega_{2} \omega_{3} \omega_{4}}} \lambda_{\omega_{1}} \lambda_{\omega_{2}} \lambda_{\omega_{3}} \lambda_{\omega_{4}} c_{\omega_{1}}^{\alpha} c_{\omega_{2}}^{\beta} \bar{c}_{\omega_{3}}^{\gamma} \bar{c}_{\omega_{4}}^{\delta} \bar{f}_{\omega_{1}}^{\alpha} \bar{f}_{\omega_{2}}^{\beta} f_{\omega_{3}}^{\gamma} f_{\omega_{4}}^{\delta} \\
& =\frac{Z_{\mathrm{imp}}}{4} \sum_{\substack{\alpha \\
\omega \omega^{\prime} \nu}} \lambda_{\omega+\nu} \lambda_{-\omega} \lambda_{-\omega^{\prime}} \lambda_{\omega^{\prime}+\nu}\left\langle c_{\omega+\nu}^{\alpha} c_{-\omega}^{\alpha} \bar{c}_{-\omega^{\prime}}^{\alpha} \bar{c}_{\omega^{\prime}+\nu}^{\alpha}\right\rangle_{\mathrm{imp}}^{U} \bar{f}_{\omega+\nu}^{\alpha} \bar{f}_{-\omega}^{\alpha} f_{-\omega^{\prime}}^{\alpha} f_{\omega^{\prime}+\nu}^{\alpha} \\
& +\frac{Z_{\text {imp }}}{4} \sum_{\substack{\alpha \beta \\
\omega \omega^{\prime} \nu}} \lambda_{\omega+\nu} \lambda_{-\omega} \lambda_{-\omega^{\prime}} \lambda_{\omega^{\prime}+\nu}\left\langle c_{\omega+\nu}^{\alpha} c_{-\omega}^{\beta} \bar{c}_{-\omega^{\prime}}^{\beta} \bar{c}_{\omega^{\prime}+\nu}^{\alpha}\right\rangle_{\mathrm{imp}}^{V=\bar{f}_{\omega+\nu}^{\alpha}} \bar{f}_{-\omega}^{\beta} f_{-\omega^{\prime}}^{\beta} f_{\omega^{\prime}+\nu}^{\alpha} \\
& +\frac{Z_{\mathrm{imp}}}{4} \sum_{\substack{\alpha \beta \\
\omega \omega^{\prime} \nu}} \lambda_{\omega+\nu} \lambda_{-\omega} \lambda_{-\omega^{\prime}} \lambda_{\omega^{\prime}+\nu}\left\langle c_{\omega+\nu}^{\alpha} c_{-\omega}^{\beta} \bar{c}_{-\omega^{\prime}}^{\alpha} \bar{c}_{\omega^{\prime}+\nu}^{\beta}\right\rangle_{\mathrm{imp}}^{V \times} \bar{f}_{\omega+\nu}^{\alpha} \bar{f}_{-\omega}^{\beta} f_{-\omega^{\prime}}^{\alpha} f_{\omega^{\prime}+\nu}^{\beta} \\
& =\frac{Z_{\mathrm{imp}}}{4} \sum_{\substack{\alpha \\
\omega \omega^{\prime} \nu}} \lambda_{\omega+\nu} \lambda_{-\omega} \lambda_{-\omega^{\prime}} \lambda_{\omega^{\prime}+\nu}\left\langle c_{\omega+\nu}^{\alpha} c_{-\omega}^{\alpha} \bar{c}_{-\omega^{\prime}}^{\alpha} \bar{c}_{\omega^{\prime}+\nu}^{\alpha}\right\rangle_{\mathrm{imp}}^{U} \bar{f}_{\omega+\nu}^{\alpha} \bar{f}_{-\omega}^{\alpha} f_{-\omega^{\prime}}^{\alpha} f_{\omega^{\prime}+\nu}^{\alpha} \\
& +\frac{Z_{\text {imp }}}{2} \sum_{\substack{\alpha \beta \\
\omega \omega^{\prime} \nu}} \lambda_{\omega+\nu} \lambda_{-\omega} \lambda_{-\omega^{\prime}} \lambda_{\omega^{\prime}+\nu}\left\langle c_{\omega+\nu}^{\alpha} c_{-\omega}^{\beta} \bar{c}_{-\omega^{\prime}}^{\beta} \bar{c}_{\omega^{\prime}+\nu}^{\alpha}\right\rangle_{\mathrm{imp}}^{V=} \bar{f}_{\omega+\nu}^{\alpha} \bar{f}_{-\omega}^{\beta} f_{-\omega^{\prime}}^{\beta} f_{\omega^{\prime}+\nu}^{\alpha} .
\end{aligned}
$$

Here, $\langle\ldots\rangle_{\text {imp }}^{V=(\times)}$ denotes an average in the horizontal (crossed) channel where no Hubbard interaction lines connect Green function lines on the two-particle level, i. e. all the two-particle diagrams one obtains for $U=0$ and $V>0$. Corresponding diagrams are shown in a) of fig. 4.1. The diagrams in the top row of a), b) and c) correspond to the horizontal channel, the diagrams in the lower rows belong to the crossed channel. 
a)
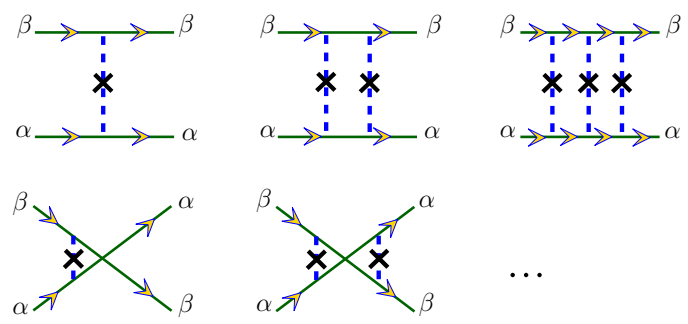

b)
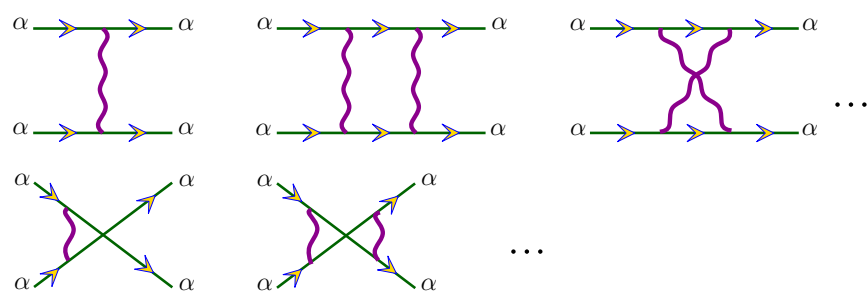

c)
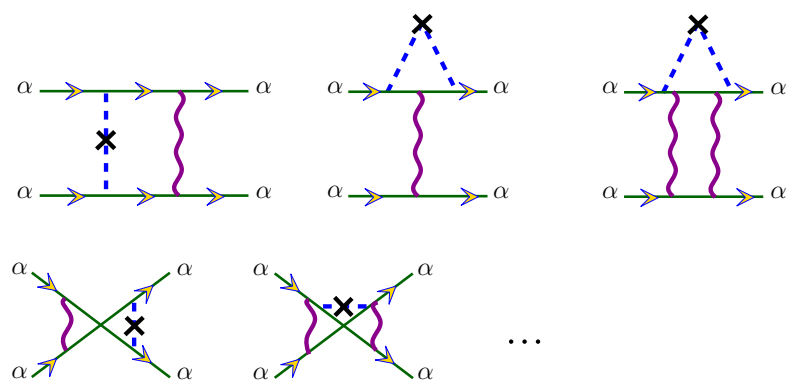

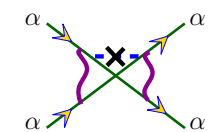

Figure 4.1: Different contributions to the vertex function. Depending on the spin configuration not all shown diagrams may be possible. The contributions in the top row of a) are part of $\langle c c \bar{c} \bar{c}\rangle^{V=}$ and the contributions in the bottom row of a) are part of $\langle c c \bar{c} \bar{c}\rangle^{V \times}$. These two expectation values also contain the unconnected two-particle Green functions, which are not depicted in the above. The contributions in b) and c) are part of $\langle c c \bar{c} \bar{c}\rangle^{U}$. Note that the Green function lines denote dressed single-particle Green functions. (Based on figure by S.-X. Yang) 
$\langle\ldots\rangle_{\text {imp }}^{U}$ denotes an average, where the two Green functions involved are connected by at least one Hubbard interaction line that fixes replica indices. Alternatively, one can describe $\langle\ldots\rangle_{\mathrm{imp}}^{U}$ as containing all the additional diagrams that are created on the two-particle level when the Hubbard interaction $U$ is turned on. Example diagrams are shown in b) and c) of fig. 4.1.

The final expression in eq. (4.23) is obtained as follows. From eq. 4.23a to eq. (4.23b) it was used that only six from the possible sixteen terms of the product can survive the integration. Then the $c$ - and $f$-operators are commuted and relabeled such that only one term is left. Equation (4.23d follows by integrating out the $c$ electrons.

Evaluating the replica indices requires some care as contributions with Hubbard interactions are different from contributions without. Hubbard interaction lines fix all replica indices as the Hubbard interaction does not act between different replicas. This leads to the first line of eq. (4.23d and explains the need to define $\langle\ldots\rangle_{\mathrm{imp}}^{U}$. $\langle\ldots\rangle_{\text {imp }}^{V=(\times)}$ contains terms without Hubbard interactions, thus replica indices are only fixed by electron travel. Two connections are possible: the one in the second line of eq. (4.23d corresponds to the horizontal channel and the one in the third line corresponds to the crossed channel.

For the final expression, the $c$ - and $f$-numbers are commuted and the frequencies relabeled such that only one term is left for the disorder contribution. We opted for the horizontal channel because in the following it will be related to the disorder channel of the two-particle vertex, which has no crossed contribution. Time-translational invariance was used for the frequency indices. The separation of the two-particle Green function in a purely disordered part and the rest is necessary for the derivation of the formalism. Later on, it can be convenient to combine both contributions to the dual potential for calculations. Reintroducing the crossed channel for the disorder part can facilitate calculations as well.

We continue with expanding the right hand side of eq. 4.18). To this end, the ansatz

$$
V_{d}=a_{2} \bar{f}^{\alpha} f^{\beta}+a_{4} \bar{f}^{\alpha} \bar{f}^{\beta} f^{\gamma} f^{\delta}+\ldots
$$

is used. In principle, one has to go up to infinite order in $\bar{f}$ and $f$ and then the mapping to the dual degrees of freedom is exact. However, for practical reasons, we will limit ourselves to second order.

The expansion reads

$$
\begin{aligned}
Z_{\mathrm{imp}} e^{-\sum_{\omega \alpha} \lambda_{\omega}^{2} g(\omega) \bar{f}_{\omega}^{\alpha} f_{\omega}^{\alpha}-V_{d}\left[\bar{f}^{\alpha}, f^{\beta}\right]} & =Z_{\mathrm{imp}}\left(1-\sum_{\alpha \omega} \lambda_{\omega}^{2} g(\omega) \bar{f}_{\omega}^{\alpha} f_{\omega}^{\alpha}\right. \\
& \left.+\frac{1}{2} \sum_{\alpha \beta \omega \omega^{\prime}} \lambda_{\omega}^{2} \lambda_{\omega^{\prime}}^{2} g(\omega) g\left(\omega^{\prime}\right) \bar{f}_{\omega}^{\alpha} f_{\omega}^{\alpha} \bar{f}_{\omega^{\prime}}^{\beta} f_{\omega^{\prime}}^{\beta}-V_{d} \pm \ldots\right) .
\end{aligned}
$$


The ansatz for $V_{d}$ is inserted and the coefficients for the dual potential are read off by comparing order by order. One finds that

$$
\begin{aligned}
a_{2}= & 0 \\
a_{4}= & -\frac{1}{4} \lambda_{\omega_{1}} \lambda_{\omega_{2}} \lambda_{\omega_{3}} \lambda_{\omega_{4}}\left\langle c^{\alpha} c^{\alpha} \bar{c}^{\alpha} \bar{c}^{\alpha}\right\rangle_{\mathrm{imp}}^{U} \\
& -\frac{1}{2} \lambda_{\omega_{1}} \lambda_{\omega_{2}} \lambda_{\omega_{3}} \lambda_{\omega_{4}} \delta_{\alpha \beta}\left\langle c^{\alpha} c^{\beta} \bar{c}^{\beta} \bar{c}^{\alpha}\right\rangle_{\mathrm{imp}}^{V=} \\
& +\frac{1}{2} \lambda_{\omega_{1}}^{2} \lambda_{\omega_{2}}^{2} g_{\mathrm{imp}}^{\alpha}\left(\omega_{1}\right) g_{\mathrm{imp}}^{\alpha}\left(\omega_{2}\right) \delta_{\omega_{1} \omega_{3}} \delta_{\omega_{2} \omega_{4}} \delta_{\alpha \beta} .
\end{aligned}
$$

Next, $\left\langle c_{\omega+\nu}^{\alpha} c_{-\omega}^{\alpha} \bar{c}_{-\omega^{\prime}}^{\alpha} \bar{c}_{\omega^{\prime}+\nu}^{\alpha}\right\rangle_{\text {imp }}^{U}$ and $\left\langle c_{\omega+\nu}^{\alpha} c_{-\omega}^{\beta} \bar{c}_{-\omega^{\prime}}^{\beta} \bar{c}_{\omega^{\prime}+\nu}^{\alpha}\right\rangle_{\text {imp }}^{V=}$ are examined in more detail such that we know how to calculate them. Both terms contribute to the twoparticle Green function of the replicated system. This is clear from eq. 4.23 which implies

$$
\left\langle c_{\omega+\nu}^{\alpha} c_{-\omega}^{\beta} \bar{c}_{-\omega^{\prime}}^{\beta} \bar{c}_{\omega^{\prime}+\nu}^{\alpha}\right\rangle_{\mathrm{imp}}=2\left\langle c_{\omega+\nu}^{\alpha} c_{-\omega}^{\beta} \bar{c}_{-\omega^{\prime}}^{\beta} \bar{c}_{\omega^{\prime}+\nu}^{\alpha}\right\rangle_{\mathrm{imp}}^{V=}+\left\langle c_{\omega+\nu}^{\alpha} c_{-\omega}^{\alpha} \bar{c}_{-\omega^{\prime}}^{\alpha} \bar{c}_{\omega^{\prime}+\nu}^{\alpha}\right\rangle_{\mathrm{imp}}^{U} \delta_{\alpha \beta},
$$

and the definition of the particle-particle Green function

$$
\chi^{p p}\left(\omega, \omega^{\prime}, \nu\right)=\left\langle c_{\omega+\nu} c_{-\omega} \bar{c}_{-\omega^{\prime}} \bar{c}_{\omega^{\prime}+\nu}\right\rangle .
$$

Taking into account the third contribution in eq. (4.27), $\lambda=g^{-1}$ seems a natural choice as the dual potential then becomes a two-particle vertex function.

$\left\langle c_{\omega+\nu}^{\alpha} c_{-\omega}^{\beta} \bar{c}_{-\omega^{\prime}}^{\beta} \bar{c}_{\omega^{\prime}+\nu}^{\alpha}\right\rangle_{\mathrm{imp}}^{V}$ is particularly easy, as this is the disordered particle-particle Green function. Combining it with the third contribution in eq. 4.27) yields

$$
\gamma^{v p}=\frac{\left\langle c^{\alpha} c^{\beta} \bar{c}^{\beta} \bar{c}^{\alpha}\right\rangle_{\mathrm{imp}}^{V=}-g_{\mathrm{imp}}^{\alpha} g_{\mathrm{imp}}^{\alpha} \delta_{\alpha \beta}}{g_{\mathrm{imp}}^{\alpha} g_{\mathrm{imp}}^{\beta} g_{\mathrm{imp}}^{\beta} g_{\mathrm{imp}}^{\alpha}}
$$

as one coefficient for the dual potential. $\left\langle c_{\omega+\nu}^{\alpha} c_{-\omega}^{\alpha} \bar{c}_{-\omega^{\prime}}^{\alpha} \bar{c}_{\omega^{\prime}+\nu}^{\alpha}\right\rangle_{\text {imp }}^{U}$ is obtained from the disorder-averaged particle-particle Green function by subtracting the disorderaveraged unconnected particle-particle Green functions

$$
\begin{aligned}
\left\langle c_{\omega+\nu}^{\alpha} c_{-\omega}^{\alpha} \bar{c}_{-\omega^{\prime}}^{\alpha} \bar{c}_{\omega^{\prime}+\nu}^{\alpha}\right\rangle_{\mathrm{imp}}^{U} \delta_{\alpha \beta} & =\left\langle c_{\omega+\nu}^{\alpha} c_{-\omega}^{\beta} \bar{c}_{-\omega^{\prime}}^{\gamma} \bar{c}_{\omega^{\prime}+\nu}^{\delta}\right\rangle_{\mathrm{imp}}\left(\delta_{\alpha \delta} \delta_{\beta \gamma}-\delta_{\alpha \gamma} \delta_{\beta \delta}\right) \\
& -\left\langle c^{\alpha} c^{\beta} \bar{c}^{\beta} \bar{c}^{\alpha}\right\rangle_{\mathrm{imp}}^{V=}+\left\langle c^{\alpha} c^{\beta} \bar{c}^{\alpha} \bar{c}^{\beta}\right\rangle_{\mathrm{imp}}^{V \times}
\end{aligned}
$$

and the corresponding term for the dual potential becomes

$$
\gamma^{U p}\left(\omega, \omega^{\prime}, \nu\right)=\frac{\left\langle c^{\alpha} c^{\alpha} \bar{c}^{\alpha} \bar{c}^{\alpha}\right\rangle^{U}}{g_{\mathrm{imp}}^{\alpha}(\omega+\nu) g_{\mathrm{imp}}^{\alpha}(-\omega) g_{\mathrm{imp}}^{\alpha}\left(-\omega^{\prime}\right) g_{\mathrm{imp}}^{\alpha}\left(\omega^{\prime}+\nu\right)} .
$$

In eq. 4.31) the crossing-symmetric disorder particle-particle Green function is sub- 
tracted from the particle-particle Green function, which follows from eq. 4.23d). The final expression for the dual potential reads

$$
V_{d}=-\frac{1}{4} \gamma^{U p} \bar{f}^{\alpha} \bar{f}^{\alpha} f^{\alpha} f^{\alpha}-\frac{1}{2} \gamma^{v p} \bar{f}^{\alpha} \bar{f}^{\beta} f^{\beta} f^{\alpha} .
$$

Now the dual action can be written as

$$
S_{d}\left[\bar{f}^{\alpha}, f^{\beta}\right]=-\sum_{\omega k} \bar{f}^{\alpha}\left(G_{d}^{0}\right)^{-1} f^{\alpha}+\sum_{i} V_{d}^{i}\left[\bar{f}_{i}^{\alpha}, f_{i}^{\beta}\right]
$$

with

$$
\begin{aligned}
G_{d}^{0}(\omega, k) & =-g_{\omega}\left[g_{\omega}+\left(\Delta_{\omega}-\epsilon_{k}\right)^{-1}\right]^{-1} g_{\omega} \\
& =G(\omega, k)-g_{\omega}
\end{aligned}
$$

being the bare dual Green function.

Equations 4.30 and 4.32 contain disorder-averaged quantities that depend on replica indices. The replica indices are needed for the evaluation of diagrams as they impose restrictions on the topology. It is our goal to evaluate the replica limit before actual calculations are done. Therefore, we need expressions for the dual potential after the replica limit to evaluate diagrams numerically. In the following $\langle\ldots\rangle$ denotes only quantum averages and $\{\ldots\}$ denotes the disorder-average. $g^{v}$ is the impurity Green function for an individual disorder realization.

It turns out that $\gamma^{v p}$ for the particle-particle channel is nothing but the disorderaverage of the product of two interacting impurity Green functions, thus

$$
\gamma_{\sigma \sigma^{\prime}}^{v p}(\nu)_{\omega \omega^{\prime}}=\frac{\left\{g_{\sigma}^{v}(\omega+\nu) g_{\sigma^{\prime}}^{v}(-\omega)\right\}-g_{\sigma}(\omega+\nu) g_{\sigma^{\prime}}(-\omega)}{g_{\sigma}(\omega+\nu) g_{\sigma^{\prime}}(-\omega) g_{\sigma^{\prime}}(-\omega) g_{\sigma}(\omega+\nu)} \delta_{\omega \omega^{\prime}}
$$

after the replica limit. Note that this term can only survive the replica limit as part of a diagram because there are two replica indices left before $\lim _{m \rightarrow 0}$ is evaluated.

It is sometimes convenient to have the crossed component

$$
\gamma^{v p \times}(\nu)_{\omega, \omega^{\prime}}=-\gamma^{v p}(\nu)_{\omega,-\omega^{\prime}-\nu} \delta_{\omega+\omega^{\prime}+\nu, 0}
$$

as well. For $\gamma^{v p}$ and $\gamma^{v p \times}$ all possible spin configurations have the same numerical value as we look at systems with spin symmetry. We introduce the symbols

$$
\begin{gathered}
V_{v p} \equiv \gamma_{\sigma \sigma^{\prime}}^{v p} \\
V_{v p}^{\times} \equiv \gamma_{\sigma \sigma^{\prime}}^{v p \times}
\end{gathered}
$$


for the disorder part of the dual potential. Correspondingly, one obtains

$$
\begin{aligned}
\gamma_{\sigma_{1} \sigma_{2} ; \sigma_{3} \sigma_{4}}^{U p}(\nu)_{\omega \omega^{\prime}} & =\frac{\left\{\left\langle c_{\omega+\nu, \sigma_{1}} c_{-\omega, \sigma_{2}} \bar{c}_{-\omega^{\prime}, \sigma_{3}} \bar{c}_{\omega^{\prime}+\nu, \sigma_{4}}\right\rangle_{\mathrm{imp}}\right\}}{g_{\sigma_{1}}(\omega+\nu) g_{\sigma_{2}}(-\omega) g_{\sigma_{3}}\left(-\omega^{\prime}\right) g_{\sigma_{4}}\left(\omega^{\prime}+\nu\right)} \\
& +\frac{\left\{g_{\sigma_{1}}^{v}(-\omega) g_{\sigma_{2}}^{v}\left(\omega^{\prime}+\nu\right)\left(\delta_{\sigma_{1} \sigma_{3}} \delta_{\sigma_{2} \sigma_{4}} \delta_{\omega+\omega^{\prime}+\nu, 0}-\delta_{\sigma_{1} \sigma_{4}} \delta_{\sigma_{2} \sigma_{3}} \delta_{\omega, \omega^{\prime}}\right)\right\}}{g_{\sigma_{1}}(\omega+\nu) g_{\sigma_{2}}(-\omega) g_{\sigma_{3}}\left(-\omega^{\prime}\right) g_{\sigma_{4}}\left(\omega^{\prime}+\nu\right)}
\end{aligned}
$$

for the terms with Hubbard interactions. Note that the crossing-symmetric unconnected two-particle Green function is subtracted before the disorder-average. Only a limited number of spin configurations is needed, e. g. only the pairing channel for the dual potential in the particle-particle channel

$$
V_{p}(\nu)_{\omega \omega^{\prime}}=\gamma_{\uparrow \downarrow ; \uparrow \downarrow}^{U p}(\nu)_{\omega \omega^{\prime}}
$$

It is related to the dual potential $V_{t}$ for the triplet channel via

$$
\begin{aligned}
V_{t}(\nu)_{\omega \omega^{\prime}} & =\gamma_{\uparrow \downarrow \uparrow \uparrow \downarrow}^{U p}(\nu)_{\omega \omega^{\prime}}+\gamma_{\uparrow \downarrow ; \downarrow \uparrow}^{U p}(\nu)_{\omega \omega^{\prime}} \\
& =V_{p}(\nu)_{\omega \omega^{\prime}}-V_{p}(\nu)_{\omega,-\omega^{\prime}-\nu} \\
& =V_{p}(\nu)_{\omega \omega^{\prime}}+V_{p}^{\prime}(\nu)_{\omega, \omega^{\prime}} .
\end{aligned}
$$

Crossing symmetry was used in the above. The other two components of the triplet channel are $\gamma_{\uparrow \uparrow ; \uparrow \uparrow}^{U p}$ and $\gamma_{\downarrow \downarrow ; \downarrow \downarrow \downarrow}^{U p}$. All three contributions are equal due to $\mathrm{SU}(2)$ symmetry.

For higher-order approximations the particle-particle channel and particle-hole channel are not equivalent. Therefore the dual potential for the particle-hole channel is needed. It is given by

$$
\gamma_{\sigma \sigma^{\prime}}^{v}(\nu)_{\omega \omega^{\prime}}=\frac{\left\{g_{\sigma}^{v}(\omega+\nu) g_{\sigma^{\prime}}^{v}(\omega)\right\}-g_{\sigma}(\omega+\nu) g_{\sigma^{\prime}}(\omega)}{g_{\sigma}(\omega+\nu) g_{\sigma^{\prime}}(\omega) g_{\sigma^{\prime}}(\omega) g_{\sigma}(\omega+\nu)} \delta_{\omega \omega^{\prime}}
$$

for the purely disordered part and

$$
\begin{aligned}
\gamma_{\sigma_{1} \sigma_{2} ; \sigma_{3} \sigma_{4}}^{U}(\nu)_{\omega \omega^{\prime}} & =\frac{\left\{\left\langle c_{\omega+\nu, \sigma_{1}} \bar{c}_{\omega, \sigma_{2}} c_{\omega^{\prime}, \sigma_{3}} \bar{c}_{\omega^{\prime}+\nu, \sigma_{4}}\right\rangle_{\mathrm{imp}}\right\}}{g_{\sigma_{1}}(\omega+\nu) g_{\sigma_{2}}(\omega) g_{\sigma_{3}}\left(\omega^{\prime}\right) g_{\sigma_{4}}\left(\omega^{\prime}+\nu\right)} \\
& +\frac{\left\{g_{\sigma_{3}}^{v}(\omega) g_{\sigma_{1}}^{v}\left(\omega^{\prime}+\nu\right)\left(\delta_{\sigma_{1} \sigma_{4}} \delta_{\sigma_{2} \sigma_{3}} \delta_{\omega, \omega^{\prime}}-\delta_{\sigma_{1} \sigma_{2}} \delta_{\sigma_{3} \sigma_{4}} \delta_{\nu, 0}\right)\right\}}{g_{\sigma_{1}}(\omega+\nu) g_{\sigma_{2}}(\omega) g_{\sigma_{3}}\left(\omega^{\prime}\right) g_{\sigma_{4}}\left(\omega^{\prime}+\nu\right)}
\end{aligned}
$$

for the part containing Hubbard interactions. Here, it is convenient to introduce the vertical disorder potential

$$
V_{v}^{\|}=V_{v}\left(\omega-\omega^{\prime}\right)_{\omega^{\prime}, \omega^{\prime}} \delta_{\nu, 0},
$$


where $V_{v} \equiv \gamma_{\sigma \sigma^{\prime}}^{v}$. For the interacting part we use

$$
V_{d / m}(\nu)_{\omega \omega^{\prime}}=\gamma_{\uparrow \uparrow ; \uparrow \uparrow}^{U}(\nu)_{\omega \omega^{\prime}} \pm \gamma_{\uparrow \uparrow ; \downarrow \downarrow}^{U}(\nu)_{\omega \omega^{\prime}} .
$$

These are the density and magnetic channels. A more detailed discussion of twoparticle vertices is presented in appendix $\mathrm{D}$.

This concludes the introduction of the dual degrees of freedom. The bare dual Green function and the dual potential define the dual lattice problem.

\subsection{Perturbation Theory for the Self-Energy}

In this section the dual self-energy is calculated by means of perturbation theory. In section 4.2.1 the evaluation of the replica limit is discussed and the Feynman rules for the self-energy are given. Additionally, the Schwinger-Dyson equation is introduced, which relates the self-energy to the vertex function and serves as a starting point for the second order and fluctuation exchange (FLEX) approximations. In section 4.2.2 the first and second order approximations are calculated. Section 4.2 .3 introduces the FLEX approximation which takes into account ladder diagrams up to infinite order.

We start with the first order diagram which serves as an example to illustrate how diagrams are evaluated. The workhorse in this thesis is the second order approximation as it adds non-local correlations but is not susceptible to divergences as FLEX. FLEX usually leads to stronger renormalizations of the Green function than the second order approximation. In practice, it provides better values for the antiferromagnetic transition temperature but contributions from FLEX diverge if the leading eigenvalue of the Bethe-Salpeter equation becomes greater or equal to one. The Bethe-Salpeter equation is introduced and used in section 4.2.3.

\subsubsection{Replica Limit and Feynman Rules for Self-Energy Diagrams}

When we want to obtain self-energy diagrams for the dual fermions, we have to evaluate the replica limit. For diagrams with $\gamma^{U}$ this is trivial as there is no true dependence on the replica index. Diagrams that contain $\gamma^{V}$ have to be considered in more detail. This is shown for a first order diagram in fig. 4.2 and for a second order diagram in fig. 4.3. Taking the replica limit means to perform $\lim _{m \rightarrow 0} \frac{1}{m}$. Figures 4.2 and 4.3 show that diagrams that contain a closed Fermi loop do not survive the replica limit if the loop is connected to the rest of the diagram through disorder vertices alone. Examples of closed Fermi loops are given in fig. 4.4. They are not to be confused with closed loops that represent local Green functions.

We obtain the following Feynman rules for an $n$-th order diagram for the self- 


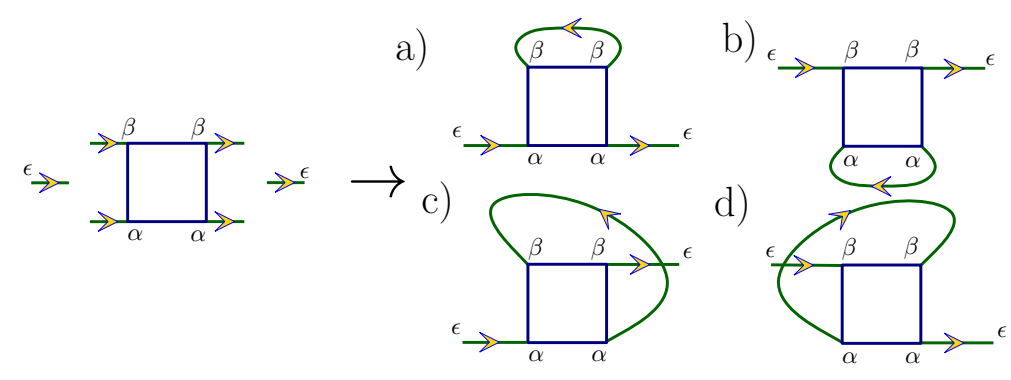

Figure 4.2: The box represents the dual potential, the green lines with arrows represent $f(\bar{f})$ operators. In first order, there are four possible ways to connect the inand outgoing $f(\bar{f})$ operators to the dual potential. For the crossing symmetric component of the dual potential all replica indices are fixed to $\epsilon$, because the Hubbard interaction is local within a replica and electrons travel only within one replica. Therefore only one free replica index is left and the replica sum is of order $m$. The diagrams will survive the replica limit. Because of the crossing symmetry of the interaction, all four contributions a)-d) give the same result. This cancels the factor $\frac{1}{4}$ in the dual potential. For the crossing asymmetric component, diagrams a) and b) are not possible, because top and bottom are of the diagram are only connected by the effective disorder interaction which connects different replica. The replica sum is of order $m^{2}$ and does not survive the replica limit. For diagrams c) and d), the internal Green function fixes the replica indices and the replica sum will be of order $m$. Thus, the diagram survives the replica limit. The two diagrams are equivalent and cancel the factor $\frac{1}{2}$ in the dual potential.

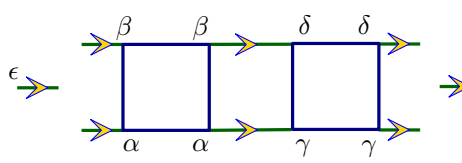

a)

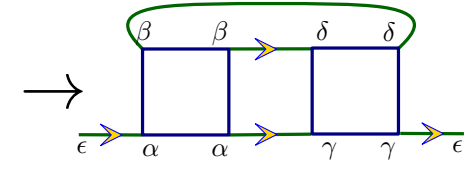

b)

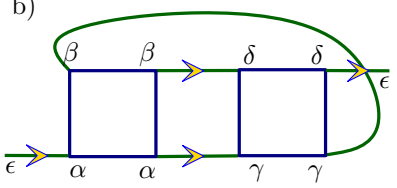

Figure 4.3: In second order up to sixteen connections are possible, depending on whether all internal Green function lines are distinguishable. Two of these diagrams are shown in this figure. All possibilities are equivalent to one of the two chosen above. If one of the vertices is crossing-symmetric it is enough to pick one of the two as they are related by crossing symmetry. In this case, only one free replica index remains as the crossing-symmetric vertices have only one free replica index. If both vertices are not crossing-symmetric both diagrams have to be taken into account as they are not equivalent. In this case, only diagram b) survives the replica limit. Diagram a) has two free replica indices remaining. Both times all prefactors cancel up to a factor $\frac{1}{2}$ for two indistinguishable Green function lines. This generalizes to $\frac{1}{k !}$ for $k$ indistinguishable lines in higher orders. 

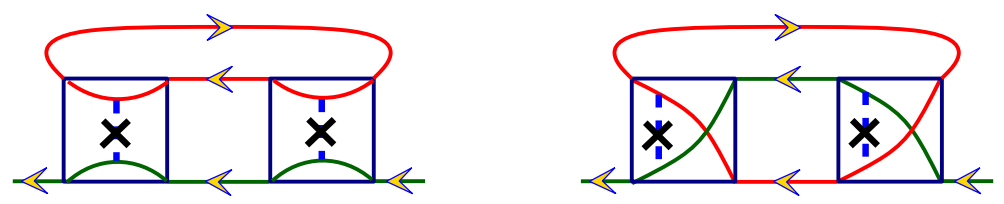

Figure 4.4: Two diagrams with closed Fermi loops that have a free replica index. The red line shows the closed Fermi loop. The example on the right uses the crossed disorder vertex. It is not part of the dual potential as it has been derived, but at times it can be convenient to add the crossed contribution back to the dual potential.

energy:

1. Draw $n$ boxes and connect them in all topologically different ways via directed lines. Exactly two corners must have no connections.

2. Associate a dual Green function $G^{d}$ with each line.

3. The boxes are associate with a factor $\gamma^{U}+\gamma^{V}$. Draw diagrams for every combination of $\gamma^{U}$ and $\gamma^{V}$.

4. Attach replica labels to the boxes and lines.

5. Determine which replica labels are fixed, either by $\gamma^{U}$ or by Green function lines.

6. Remove all diagrams that have more than one free replica index. These diagrams contain closed Fermi loops that are connected to the rest of the diagram only through disorder vertices. Figure 4.4 shows two examples of closed Fermi loops. Now, the replica limit has been performed and the replica labels can be removed from the diagrams.

7. Attach frequency, spin and momentum labels to each Green function line.

8. Sum over all internal frequencies, spins and momenta, obeying energy, spin and momentum conservation. 


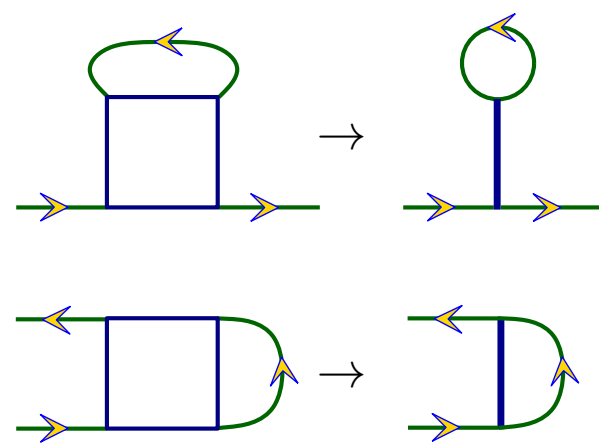

Figure 4.5: Contracting the box that represents the vertex to a single line determines whether one has a closed loop or not. In the upper example one has a closed loop, but not in the lower one.

9. Every independent frequency summation gives a factor $1 / \beta$ and every independent momentum summation a factor $1 / N$. $\beta$ is the inverse temperature and $N$ the number of dual lattice sites.

10. Multiply by $1 / k$ ! for every set of $k$ indistinguishable lines, e. g. the two internal Green function lines in the two diagrams in fig. 4.4 are indistinguishable for equal spin.

11. For every diagram determine the number of closed loops and multiply with a factor of $(-1)^{n_{l}}$ where $n_{l}$ is the number of closed loops. Closed loops are identified as follows. For interaction vertices contract the boxes to lines. Do this consistently for the whole diagram. If a Green function line ends at its starting point, i. e. it is local, it counts as a closed loop. This is illustrated in fig. 4.5. These loops are not to be confused with the closed Fermi loops illustrated in fig. 4.4 For disorder vertices the corresponding diagrams are removed by the replica limit in step 6 .

These rules are an adapted form of the rules given in [105] to accommodate the changes due to disorder.

A natural question to ask is why dual diagrams with closed Fermi loops are removed. If one constructs real fermion diagrams with the same topology, one finds that it is not possible to construct such diagrams with the Feynman rules of the disorder-averaged real fermion system. This is illustrated in fig. 4.6.

A good starting point for approximations of the self-energy is the SchwingerDyson equation. It relates the self-energy to the full vertex function. It is shown for the dual degrees of freedom in figure 4.7. For the particle-hole channel the equation 

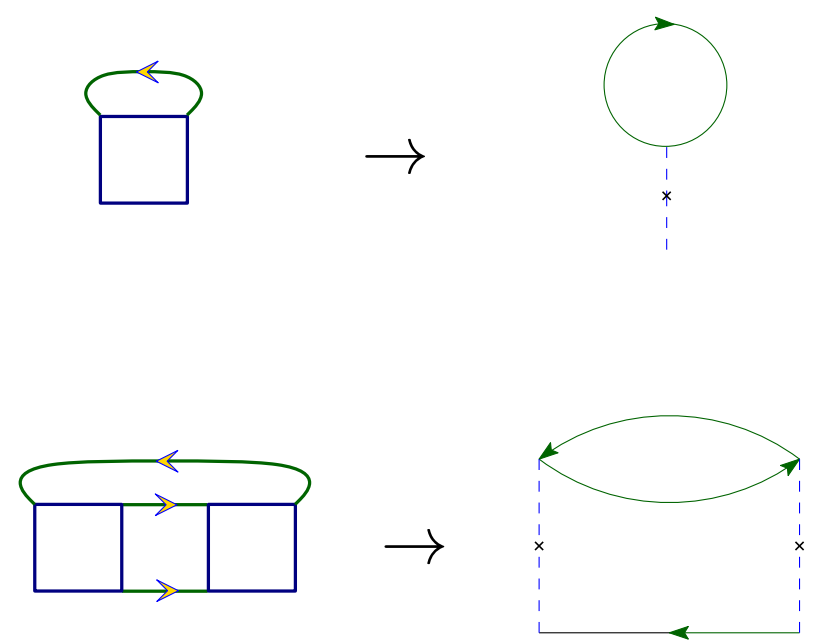

Figure 4.6: Two examples of diagrams that are removed by the replica limit. The topology of the dual fermion diagrams (left) is mapped to corresponding real fermion diagrams (right). These diagrams are not possible for real fermions. The conclusion is that the replica limit removes diagrams with a topology that is unphysical for real fermions. This fact underlines that there is a close relation between dual and real fermion excitations. Section 4.3 investigates this in more detail.

a)

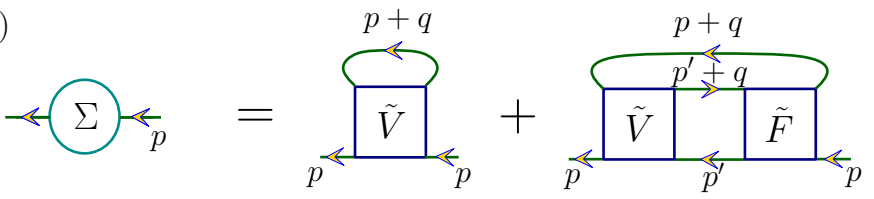

b)

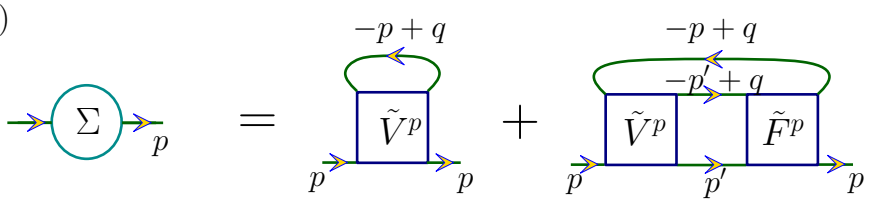

Figure 4.7: Illustration of the Schwinger-Dyson equation for the particle-hole and particleparticle channel. (Illustration by S.-X. Yang [106]) 
$\mathrm{ph}$

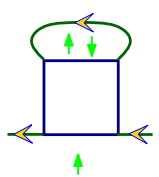

$\mathrm{pp}$

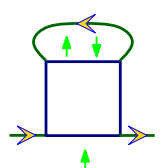

Figure 4.8: Spin configurations in first order for the particle-hole (ph) and particle-particle (pp) channel.

reads

$$
\begin{aligned}
\Sigma_{\sigma}^{p h}(\omega, k)= & -\frac{T}{N} \sum_{q \sigma^{\prime}} \tilde{V}(\nu)_{\omega ; \omega} G_{\sigma^{\prime}}^{d}(\omega+\nu, k+q) \\
& -\frac{T}{2 N} \sum_{\substack{\omega^{\prime}, k^{\prime}, \nu, q \\
\sigma^{\prime}, \sigma^{\prime \prime}, \sigma^{\prime \prime \prime}}} \tilde{V}(\nu)_{\omega, \omega^{\prime}} G_{\sigma^{\prime}}^{d}\left(\omega^{\prime}+\nu, k^{\prime}+q\right) G_{\sigma^{\prime \prime}}^{d}\left(\omega^{\prime}, k^{\prime}\right) \times \\
& \times \tilde{F}(\nu, q)_{\omega^{\prime} k^{\prime} ; \omega k} G_{\sigma^{\prime \prime \prime}}^{d}(\omega+\nu, k+q),
\end{aligned}
$$

where $\tilde{V}$ is the dual potential, i. e. the impurity vertex, and $\tilde{F}$ is the full dual vertex. Spin indices on $\tilde{V}$ and $\tilde{F}$ have been suppressed for readability. Note that for $U=0$ the bare vertex does not exist for the real fermion system. Therefore, the SchwingerDyson equation does not exist. However, for the dual degrees of freedom the bare vertex can be defined and thus the Schwinger-Dyson equation can be derived for the dual system.

\subsubsection{First Order and Second Order Contributions}

The first order contribution has two possible spin configurations. These are shown in fig. 4.8. In first order, contributions from the particle-particle channel and particlehole channel are topologically equivalent, therefore only one contribution has to be taken into account. For the particle-particle channel it follows that

$$
\begin{aligned}
\Sigma_{p p}^{(1)}(\omega, k)= & \frac{T}{N} \sum_{\nu, q}\left(V_{p}(\nu, q)_{-\omega ;-\omega}+V_{t}(\nu, q)_{-\omega ;-\omega}+V_{v p}^{\times}(\nu=0, q)_{-\omega ;-\omega}\right) \times \\
& \times G^{d}(\omega+\nu, k+q) \\
= & \frac{T}{N} \sum_{\nu, q}\left(2 V_{p}(\nu, q)_{-\omega ;-\omega}+V_{p}^{\prime}(\nu, q)_{-\omega ;-\omega}+V_{v p}^{\times}(\nu=0, q)_{-\omega ;-\omega}\right) \times \\
& \times G^{d}(\omega+\nu, k+q) .
\end{aligned}
$$



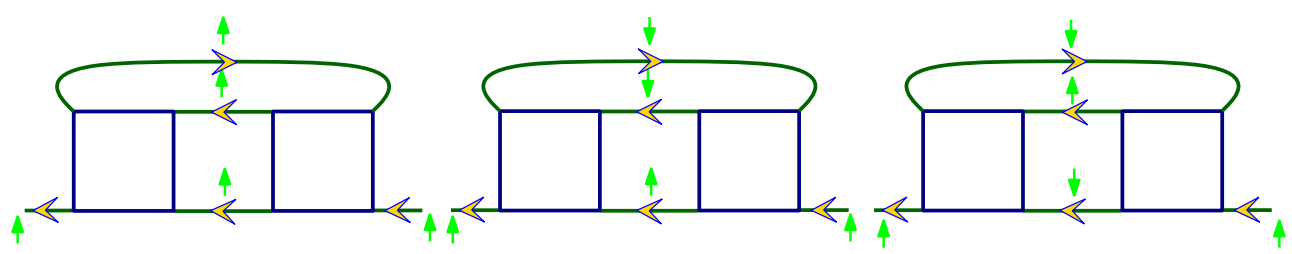

Figure 4.9: The three different spin configurations for the particle-particle channel. The first contribution has a symmetry factor of $\frac{1}{2 !}$ due to the two indistinguishable Green function lines. This is an example of Feynman rule 10. The second and third contributions are topologically equivalent, therefore there is a symmetry factor $\frac{1}{2}$.

For the particle-hole channel one obtains

$\Sigma_{p h}^{(1)}(\omega, k)=-\frac{T}{N} \sum_{\nu, q}\left(\frac{1}{2} V_{d}(\nu, q)_{\omega ; \omega}+\frac{3}{2} V_{m}(\nu, q)_{\omega ; \omega}+V_{v}^{\|}(\nu=0, q)_{\omega ; \omega}\right) G^{d}(\omega+\nu, k+q)$.

It can be shown that a restriction to first order does not change the DMFT result (cf. [105]) as the exact DMFT solution gives $\Sigma^{(1)} \equiv 0$.

In the interest of obtaining the second order contribution for the self-energy one approximates $\tilde{F}$ by $\tilde{V}$ in the Schwinger-Dyson equation. To calculate the self-energy, it is helpful to introduce the vertex ladder. For the particle-particle pairing channel the vertex ladder is defined as

$$
\begin{aligned}
\Phi_{p}^{(2)}(\nu, q)_{\omega k ; \omega^{\prime} k^{\prime}} & =\sum_{\omega^{\prime \prime}} \tilde{V}_{p}(\nu)_{\omega \omega^{\prime \prime}} \bar{\chi}_{0}^{p p}(\nu, q)_{\omega^{\prime \prime}} \tilde{V}_{p}(\nu)_{\omega^{\prime \prime} \omega^{\prime}} \\
& -\sum_{\omega^{\prime \prime}} V_{v p}(\nu)_{\omega} \delta_{\omega ; \omega^{\prime \prime}} \bar{\chi}_{0}^{p p}(\nu, q)_{\omega^{\prime \prime}} V_{v p}(\nu)_{\omega^{\prime}} \delta_{\omega^{\prime \prime} ; \omega^{\prime}}
\end{aligned}
$$

in second order. The name vertex ladder comes from the included diagrams which look like ladders with the vertices as rungs. Here, $\tilde{V}_{p}$ is defined as

$$
\tilde{V}_{p}(\nu)_{\omega ; \omega^{\prime}}=V_{p}(\nu)_{\omega ; \omega^{\prime}}+V_{v p}(\nu)_{\omega} \delta_{\omega ; \omega^{\prime}} .
$$

Further, the $k$-averaged unconnected two-particle Green function is

$$
\bar{\chi}_{0}^{p p}(\nu, q)_{\omega}=\frac{T}{N} \sum_{k} \chi_{0}^{p p}(\nu, q)_{\omega k}
$$

with the unconnected two-particle Green function

$$
\chi_{0}^{p p}(\nu, q)_{\omega k}=-G^{d}(\omega+\nu, k+q) G^{d}(-\omega,-k) .
$$

The second term in equation 4.50 removes the unphysical disorder contribution. 
This is due to the replica limit. For the triplet channel, i. e. the left diagram in fig. 4.9, one obtains

$$
\Phi_{t}^{(2)}(\nu, q)=\sum_{\omega^{\prime \prime}} \tilde{V}_{t}(\nu)_{\omega \omega^{\prime \prime}} \bar{\chi}_{0}^{p p}(\nu, q)_{\omega^{\prime \prime}} \tilde{V}_{t}(\nu)_{\omega^{\prime \prime} \omega^{\prime}}-\sum_{\omega^{\prime \prime}} V_{v p}(\nu)_{\omega} \bar{\chi}_{0}^{p p}(\nu, q)_{\omega^{\prime \prime}} V_{v p}(\nu)_{\omega^{\prime}} \delta_{\omega ; \omega^{\prime}}
$$

where

$$
\tilde{V}_{t}=\tilde{V}_{p}+\tilde{V}_{p}^{\prime}
$$

and

$$
X^{\prime}(\nu)_{\omega ; \omega^{\prime}}:=-X(\nu)_{\omega ;-\omega^{\prime}-\nu}
$$

defines a rotation for some vertex function $X$. With this, $\Phi_{t}^{(2)}$ becomes

$$
\begin{aligned}
\Phi_{t}^{(2)}(\nu, q)_{\omega k ; \omega^{\prime} k^{\prime}}= & \sum_{\omega^{\prime \prime}} \tilde{V}_{p}(\nu)_{\omega \omega^{\prime \prime}} \bar{\chi}_{0}^{p p}(\nu, q)_{\omega^{\prime \prime}} \tilde{V}_{p}(\nu)_{\omega^{\prime \prime} \omega^{\prime}} \\
& +\sum_{\omega^{\prime \prime}} \tilde{V}_{p}(\nu)_{\omega \omega^{\prime \prime}} \bar{\chi}_{0}^{p p}(\nu, q)_{\omega^{\prime \prime}} \tilde{V}_{p \prime}(\nu)_{\omega^{\prime \prime} \omega^{\prime}} \\
& -\sum_{\omega^{\prime \prime}} V_{v p}(\nu)_{\omega} \delta_{\omega ; \omega^{\prime \prime}} \bar{\chi}_{0}^{p p}(\nu, q)_{\omega^{\prime \prime}} V_{v p}(\nu)_{\omega^{\prime}} \delta_{\omega^{\prime \prime} ; \omega^{\prime}} \\
= & \Phi_{p}^{(2)}(\nu, q)_{\omega k ; \omega^{\prime} k^{\prime}}+\Phi_{p}^{(2) \prime}(\nu, q)_{\omega k ; \omega^{\prime} k^{\prime}}-\Phi_{v p}^{(2)}(\nu, q)_{\omega k ; \omega^{\prime} k^{\prime}}
\end{aligned}
$$

Here, we defined

$$
\begin{aligned}
\Phi_{p}^{(2)}(\nu, q)_{\omega k ; \omega^{\prime} k^{\prime}} & =\sum_{\omega^{\prime \prime}} \tilde{V}_{p}(\nu)_{\omega \omega^{\prime \prime}} \bar{\chi}_{0}^{p p}(\nu, q)_{\omega^{\prime \prime}} \tilde{V}_{p}(\nu)_{\omega^{\prime \prime} \omega^{\prime}} \\
\Phi_{p}^{(2) \prime}(\nu, q)_{\omega k ; \omega^{\prime} k^{\prime}} & =\sum_{\omega^{\prime \prime}} \tilde{V}_{p}(\nu)_{\omega \omega^{\prime \prime}} \bar{\chi}_{0}^{p p}(\nu, q)_{\omega^{\prime \prime}} \tilde{V}_{p \prime}(\nu)_{\omega^{\prime \prime} \omega^{\prime}} \\
\Phi_{v p}^{(2)}(\nu, q)_{\omega k ; \omega^{\prime} k^{\prime}} & =\sum_{\omega^{\prime \prime}} V_{v p}(\nu)_{\omega} \delta_{\omega ; \omega^{\prime \prime}} \bar{\chi}_{0}^{p p}(\nu, q)_{\omega^{\prime \prime}} V_{v p}(\nu)_{\omega^{\prime}} \delta_{\omega^{\prime \prime} ; \omega^{\prime}} .
\end{aligned}
$$

Therefore, the total vertex ladder for second order in the particle-particle channel reads

$$
\Phi_{p p}^{(2)}=2\left(\Phi_{p}^{(2)}-\Phi_{v p}^{(2)}\right)+\Phi_{p}^{(2) \prime}
$$

The self-energy is calculated as

$$
\Sigma_{p p}^{(2)}(\omega, k)=\frac{T}{N} \sum_{\nu, q} \Phi_{p p}^{(2)}(\nu, q)_{-\omega ;-\omega} G^{d}(\omega+\nu, k+q) .
$$

Alternatively, it is possible to calculate the second order self-energy from the particlehole channel. This is equivalent to the particle-particle channel because the diagrams are topologically equivalent.

Three different spin configurations are possible for the particle-hole channel. These are shown in fig. 4.10. The vertices can be expressed in terms of the magnetic and 

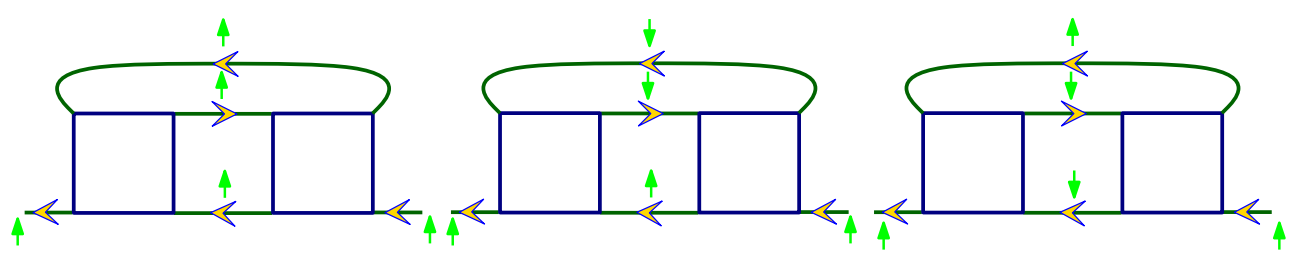

Figure 4.10: The three different spin configurations for the particle-hole channel. The second and third contributions are topologically the same, therefore there is a symmetry factor $\frac{1}{2}$. The first contribution has the same symmetry factor due to the two indistinguishable Green function lines.

charge channels. To take into account disorder in a natural fashion we define

$$
\begin{aligned}
\tilde{V}_{d}(\nu)_{\omega ; \omega^{\prime}} & =V_{d}(\nu)_{\omega ; \omega^{\prime}}+V_{v}(\nu)_{\omega} \delta_{\omega ; \omega^{\prime}} \\
\tilde{V}_{m}(\nu)_{\omega ; \omega^{\prime}} & =V_{m}(\nu)_{\omega ; \omega^{\prime}}+V_{v}(\nu)_{\omega} \delta_{\omega ; \omega^{\prime}}
\end{aligned}
$$

For the particle-hole channel the $k$-averaged unconnected two-particle Green function is

$$
\bar{\chi}_{0}^{p h}(\nu, q)_{\omega}=\frac{T}{N} \sum_{k} \chi_{0}^{p h}(\nu, q)_{\omega k}
$$

with the unconnected two-particle Green function

$$
\chi_{0}^{p h}(\nu, q)_{\omega k}=G^{d}(\omega+\nu, k+q) G^{d}(\omega, k) .
$$

With these definitions it follows that

$$
\begin{aligned}
\Phi_{d}^{(2)}(\nu, q)_{\omega ; \omega^{\prime}} & =\sum_{\omega^{\prime \prime}} \tilde{V}_{d}(\nu)_{\omega ; \omega^{\prime \prime}} \bar{\chi}_{0}^{p h}(\nu, q)_{\omega^{\prime \prime}} \tilde{V}_{d}(\nu)_{\omega^{\prime \prime} \omega^{\prime}} \\
& +2 \tilde{V}_{d}(\nu)_{\omega ; \omega^{\prime \prime}} \bar{\chi}_{0}^{p h}(\nu, q)_{\omega^{\prime \prime}} V_{v}^{\|}\left(\omega^{\prime \prime}-\omega^{\prime}\right)_{\omega^{\prime}} \delta_{\nu, 0} \\
& +2 V_{v}^{\|}\left(\omega-\omega^{\prime \prime}\right)_{\omega^{\prime \prime}} \delta_{\nu, 0} \bar{\chi}_{0}^{p h}(\nu, q)_{\omega^{\prime \prime}} \tilde{V}_{d}(\nu)_{\omega^{\prime \prime} \omega^{\prime}} \\
\Phi_{m}^{(2)}(\nu, q)_{\omega ; \omega^{\prime}} & =\sum_{\omega^{\prime \prime}} \tilde{V}_{m}(\nu)_{\omega ; \omega^{\prime \prime}} \bar{\chi}_{0}^{p h}(\nu, q)_{\omega^{\prime \prime}} \tilde{V}^{m}(\nu)_{\omega^{\prime \prime} \omega^{\prime}} \\
\Phi_{v}^{(2)}(\nu, q)_{\omega ; \omega^{\prime}} & =\sum_{\omega^{\prime \prime}} V_{v}^{=}(\nu)_{\omega} \delta_{\omega ; \omega^{\prime \prime}} \bar{\chi}_{0}^{p h}(\nu, q)_{\omega^{\prime \prime}} V_{v}^{=}(\nu)_{\omega} \delta_{\omega^{\prime \prime} ; \omega^{\prime}} \\
& +V_{v}^{\|}\left(\omega-\omega^{\prime \prime}\right)_{\omega^{\prime \prime}} \delta_{\nu, 0} \bar{\chi}_{0}^{p h}(\nu, q)_{\omega^{\prime \prime}} V_{v}^{\|}\left(\omega^{\prime \prime}-\omega^{\prime}\right)_{\omega^{\prime}} \delta_{\nu, 0} .
\end{aligned}
$$

Then, the second order self-energy from the particle-hole channel is

$\Sigma_{p h}^{(2)}(\omega, k)=-\frac{T}{N} \sum_{\nu, q}\left(\frac{1}{4} \Phi_{d}^{(2)}(\nu, q)_{\omega ; \omega}+\frac{3}{4} \Phi_{m}^{(2)}(\nu, q)_{\omega ; \omega}-\Phi_{v}^{(2)}(\nu, q)_{\omega ; \omega}\right) G^{d}(\omega+\nu, k+q)$. 


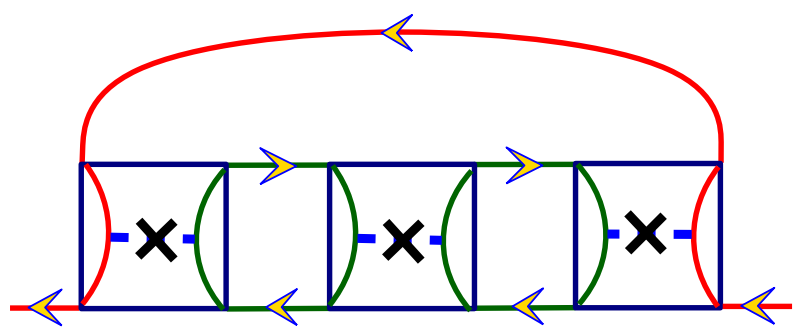

Figure 4.11: Example of an (unphysical) ring type diagram. The red line marks the way of the electron through the diagram.

\subsubsection{The FLEX Approximation}

For the FLEX approximation one has to sum all ladder and ring type diagrams, which corresponds to a combination of the Random Phase Approximation (RPA) and T-matrix approximation [108]. The contributions from the T-matrix approximation (ladder diagrams) are easily seen by replacing the boxes for the vertices by interaction lines. To see the RPA-like (ring type diagrams) contributions one can look at the unphysical diagram where one replaces all vertices by $V_{v}^{\|}$, c.f. fig. 4.11 . This diagram clearly has a ring type structure. It does not survive the replica limit, but the crossing symmetric vertices contain corresponding contributions as well. The full dual vertex can be calculated according to Bethe-Salpeter equation

$$
\tilde{F}(\nu, q)_{\omega k ; \omega^{\prime} k^{\prime}}=\tilde{V}(\nu)_{\omega ; \omega^{\prime}}+\sum_{\omega^{\prime \prime} k^{\prime \prime}} \tilde{\Gamma}(\nu, q)_{\omega k ; \omega^{\prime \prime} k^{\prime \prime}} \bar{\chi}_{0}(\nu, q)_{\omega^{\prime \prime} k^{\prime \prime}} \tilde{F}(\nu, q)_{\omega^{\prime \prime} k^{\prime \prime} ; \omega^{\prime} k^{\prime}}
$$

Here, $\tilde{\Gamma}$ is the irreducible dual fermion vertex. For the FLEX the irreducible dual vertex is approximated by the dual potential $\tilde{\Gamma} \approx \tilde{V}$. Then the equation for $\tilde{F}$ can be solved as

$$
\begin{aligned}
\tilde{F}(\nu, q)_{\omega k ; \omega^{\prime} k^{\prime}} & =\left[\frac{1}{1-\tilde{V}(\nu) \bar{\chi}_{0}(\nu, q)} \times \tilde{V}(\nu)\right]_{\omega k ; \omega^{\prime} k^{\prime}} \\
& =\left[\tilde{V}(\nu) \times \frac{1}{1-\bar{\chi}_{0}(\nu, q) \tilde{V}(\nu)}\right]_{\omega k ; \omega^{\prime} k^{\prime}} .
\end{aligned}
$$

Now we can define the vertex ladder as

$$
\Phi(\nu, q)_{\omega k ; \omega^{\prime} k^{\prime}}=\tilde{F}(\nu, q)_{\omega k ; \omega^{\prime} k^{\prime}}-\tilde{V}(\nu)_{\omega ; \omega^{\prime}} .
$$

For the calculation of the self-energy, $\Phi^{(2)}$ has to be subtracted from $\Phi$ because the second-order contribution is special and has to be treated separately. First, additional symmetry factors do exist in second order. These symmetry factors do not appear in higher order diagrams and therefore the prefactor is not suitable for the ladder summation. Second, this avoids double counting because in second order 

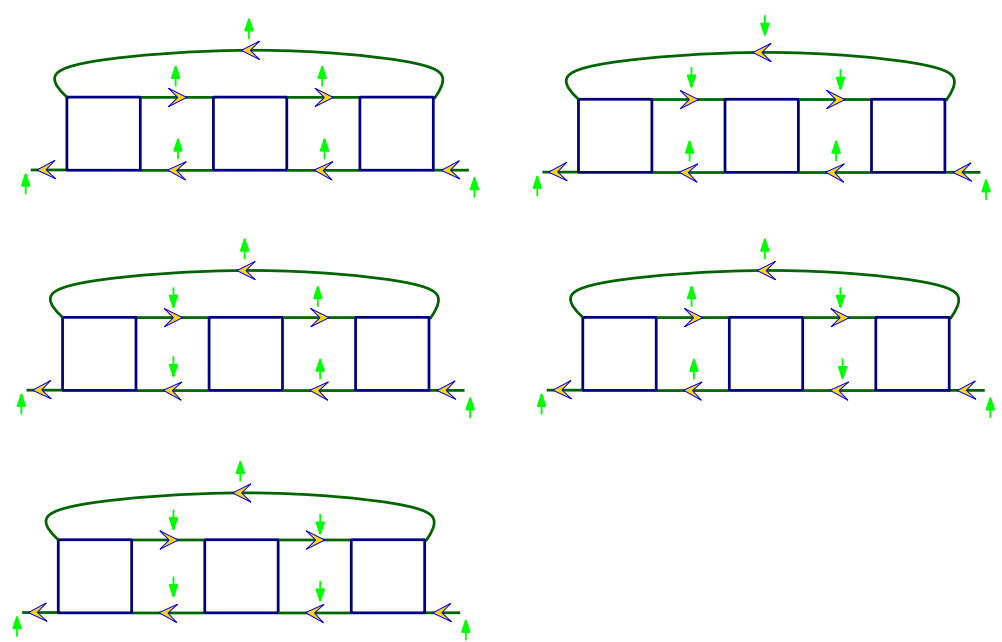

Figure 4.12: Particle-hole diagrams for the third order contributions to the FLEX.

the particle-particle and particle-hole diagrams are topologically equivalent.

For the pairing channel one obtains

$$
\tilde{F}^{p}(\nu, q)_{\omega k ; \omega^{\prime} k^{\prime}}=\left[\frac{1}{1-\tilde{V}^{p}(\nu) \bar{\chi}_{0}^{p p}(\nu, q)} \times \tilde{V}^{p}(\nu)\right]_{\omega k ; \omega^{\prime} k^{\prime}}
$$

and for the triplet channel

$$
\tilde{F}^{t}(\nu, q)_{\omega k ; \omega^{\prime} k^{\prime}}=\left[\frac{1}{1-\tilde{V}^{p}(\nu) \bar{\chi}_{0}^{p p}(\nu, q)} \times\left(\tilde{V}^{p}+\tilde{V}^{p \prime}(\nu)\right)\right]_{\omega k ; \omega^{\prime} k^{\prime}}
$$

From this the FLEX contribution to the self-energy can be calculated for the particleparticle channel as

$$
\begin{array}{r}
\sum_{p p}^{F L E X}(\omega, k)=\frac{T}{N} \sum_{\nu, q}\left[2\left(\Phi^{p}(\nu, q)_{-\omega,-\omega}-\Phi^{p(2)}-\Phi^{v p}(\nu, q)_{-\omega,-\omega}\right)\right. \\
\left.+\left(\Phi^{p \prime}(\nu, q)_{-\omega,-\omega}-\Phi^{p(2)^{\prime}}(\nu, q)_{-\omega,-\omega}\right)\right] G^{d}(\omega+\nu, k+q)
\end{array}
$$

where

$$
\begin{aligned}
\Phi_{v p}(\nu, q)_{\omega k ; \omega^{\prime} k^{\prime}} & =\left[\frac{1}{1-V_{v p}(\nu) \bar{\chi}_{0}^{p p}(\nu, q)} \times V_{v p}(\nu)\right]_{\omega k ; \omega^{\prime} k^{\prime}}-V_{v p}(\nu)_{\omega ; \omega^{\prime}} \bar{\chi}_{0}^{p p}(\nu, q) V_{v p}(\nu)_{\omega ; \omega^{\prime}} \\
& -V_{v p}(\nu)_{\omega ; \omega^{\prime}}
\end{aligned}
$$

removes the contributions that do not survive the replica limit.

Unlike for the first and second order contributions, for the FLEX one has to take 
into account both, particle-particle and particle-hole channel contributions. The diagrams from the two channels are topologically different.

The third order diagrams for the particle-hole channel are shown in fig. 4.12. One result of the replica limit is that one cannot have diagrams with only $V_{v}^{=}$that are closed in a Hartree-like fashion. Hartree-like fashion means that the Green function line connecting the left and right of the diagram connects the top left to the top right corner. In a Fock-like diagram, the connection goes from the lower right corner of the diagram to the top left. These two connections are shown in fig. 4.3. Instead of including the Fock-like diagram, one can replace one of the $V_{v}^{=}$vertices by $V_{v}^{\|}$ for the particle-hole channel. Only one term of $V_{v}^{\|}$is possible, otherwise one has a free Fermi loop. This removes all diagrams with a free replica index, because $\lim _{m \rightarrow 0} \cdots=0$ for these diagrams. Now the vertex ladder can be calculated as

$$
\begin{aligned}
\Phi_{p h}^{F L E X} & =\frac{1}{2}\left(\frac{\tilde{V}_{d}}{1-\tilde{V}_{d} \bar{\chi}_{0}^{p h}}-\tilde{V}_{d} \bar{\chi}_{0}^{p h} \tilde{V}_{d}-\tilde{V}_{d}\right) \\
& +\frac{1}{1-\tilde{V}_{d} \bar{\chi}_{0}^{p h}} V_{v}^{\|} \frac{1}{1-\bar{\chi}_{0}^{p h} \tilde{V}_{d}}-\tilde{V}_{d} \bar{\chi}_{0}^{p h} V_{v}^{\|}-V_{v}^{\|} \bar{\chi}_{0}^{p h} \tilde{V}_{d}-V_{v}^{\|} \\
& +\frac{3}{2}\left(\frac{\tilde{V}_{m}}{1-\tilde{V}_{m} \bar{\chi}_{0}^{p h}}-\tilde{V}_{m} \bar{\chi}_{0}^{p h} \tilde{V}_{m}-\tilde{V}_{m}\right) \\
& -2\left(\frac{V_{v}^{=}}{1-V_{v}^{=} \bar{\chi}_{0}^{p h}}-V_{v}^{=} \bar{\chi}_{0}^{p h} V_{v}^{=}-V_{v}^{=}\right)
\end{aligned}
$$

and the self-energy contribution reads

$$
\Sigma_{p h}^{F L E X}(\omega, k)=-\frac{T}{N} \sum_{\nu, q} \Phi_{p h}^{F L E X}(\nu, q)_{\omega ; \omega} G^{d}(\omega+\nu, k+q) .
$$

The final formula for the self-energy is

$$
\Sigma^{F L E X}=\Sigma_{p h / p p}^{(2)}+\Sigma_{p p}^{F L E X}+\Sigma_{p h}^{F L E X} .
$$

This concludes the calculation of the self-energy. The next section discusses the connection between dual and real quantities. This will enable us to formulate a self-consistency loop in section 4.4 .

\subsection{Relations between Dual and Real Quantities}

So far, the dual Green function can be calculated, but the quantities of interest are real Green functions. Fortunately, simple exact relations between dual and real quantities do exist. This section starts with the derivation of a relation between the lattice Green functions and continues with a relation between the dual and 
real vertex function. The relations obtained here are very similar to the relations derived in [105]. Similar relations do exist for higher Green functions, as well as for the corresponding cumulants and ultimately the vertex functions.

In section 4.5 vertex corrections for the conductivity are calculated using the real fermion vertex. Non-local corrections to the vertex are calculated at the level of the dual degrees of freedom and a simple relation for the vertex functions allows to translate the dual vertex to the real vertex.

The starting point for both examples is the functional

$$
F[\bar{K}, K]=\left\{\ln Z_{f} \int D[\bar{c}, c] D[\bar{f}, f] \exp \left(-S[\bar{c}, c ; \bar{f}, f]+\bar{K}_{1} f_{1}+\bar{f}_{2} K_{2}\right)\right\}
$$

in which source fields $\bar{K}$ and $K$ are introduced, summation over repeated indices is implied and $Z_{f}=\prod_{\omega k} g_{\omega}^{-2}\left(\Delta_{\omega}-\epsilon_{k}\right)$. Arabic numbers are a combined index for spin, frequency and momentum. Integrating out the $c$-degrees of freedom yields

$$
F[\bar{K}, K]=\left\{\ln \tilde{Z}_{f} \int D[\bar{f}, f] \exp \left(-S_{d}[\bar{f}, f]+\bar{K}_{1} f_{1}+\bar{f}_{2} K_{2}\right)\right\}
$$

with $\tilde{Z}_{f}=\frac{Z}{Z_{d}}$ and

$$
Z_{d}=\int D[\bar{f}, f] e^{-S_{d}[\bar{f}, f]} .
$$

Now functional derivatives like

$$
\begin{aligned}
-\left.\frac{\delta^{2} F[\bar{K}, K]}{\delta K_{2} \delta \bar{K}_{1}}\right|_{\bar{K}_{1}=K_{2}=0}= & \left\{-\frac{\delta}{\delta K} \frac{1}{Z_{d}} \int D[\bar{f}, f] f_{1} \exp \left(-S_{d}[\bar{f}, f]+\bar{K}_{1} f_{1}+\bar{f}_{2} K_{2}\right)\right\} \\
= & \left\{-\frac{1}{Z_{d}} \int D[\bar{f}, f] f_{1} \bar{f}_{2} \exp \left(-S_{d}[\bar{f}, f]+\bar{K}_{1} f_{1}+\bar{f}_{2} K_{2}\right)\right. \\
& +\frac{1}{Z_{d}^{2}} \int D[\bar{f}, f] f_{1} \exp \left(-S_{d}[\bar{f}, f]+\bar{K}_{1} f_{1}+\bar{f}_{2} K_{2}\right) \times \\
& \left.\times \int D[\bar{f}, f] \bar{f}_{2} \exp \left(-S_{d}[\bar{f}, f]+\bar{K}_{1} f_{1}+\bar{f}_{2} K_{2}\right)\right\} \\
= & \left\{-\frac{1}{Z_{d}} \int D[\bar{f}, f] f_{1} \bar{f}_{2} \exp \left(-S_{d}[\bar{f}, f]+\bar{K}_{1} f_{1}+\bar{f}_{2} K_{2}\right)\right\} \\
= & G^{d}
\end{aligned}
$$


can be taken. For the two-particle cumulant

$$
\begin{aligned}
&\left.\frac{\delta^{4} F[\bar{K}, K]}{\delta K_{4} \delta K_{3} \delta \bar{K}_{2} \delta \bar{K}_{1}}\right|_{\bar{K}_{1}=\bar{K}_{2}=K 3=K 4=0}=\lim _{m \rightarrow 0} \frac{1}{m} \frac{\delta^{4}}{\delta K_{1} \delta K_{2} \delta \bar{K}_{3} \delta \bar{K}_{4}} \tilde{Z}_{f}^{m} \times \\
& \times \int D[\bar{f}, f] \exp \left(-S_{d}[\bar{f}, f]+\bar{K}_{\mu} f_{\mu}+\bar{f}_{\nu} K_{\nu}\right) \\
&= \lim _{m \rightarrow 0} \frac{1}{m} \int D[\bar{f}, f] \sum_{\alpha \beta} f_{1}^{\alpha} f_{2}^{\beta} \bar{f}_{3}^{\gamma} \bar{f}_{4}^{\delta} \exp \left(-S_{d}[\bar{f}, f]+\bar{K}_{\mu} f_{\mu}+f_{\nu} K_{\nu}\right) \\
&= \lim _{m \rightarrow 0} \frac{1}{m} \int D[\bar{f}, f] \sum_{\alpha \beta}\left(\delta_{\alpha \beta} f_{1}^{\alpha} f_{2}^{\alpha} \bar{f}_{3}^{\alpha} \bar{f}_{4}^{\alpha}+f_{1}^{\alpha} f_{2}^{\beta} \bar{f}_{3}^{\beta} \bar{f}_{4}^{\alpha}+f_{1}^{\alpha} f_{2}^{\beta} \bar{f}_{3}^{\alpha} \bar{f}_{4}^{\beta}\right) \times \\
& \times \exp \left(-S_{d}[\bar{f}, f]+\bar{K}_{\mu} f_{\mu}+f_{\nu} K_{\nu}\right) \\
&= \lim _{m \rightarrow 0} \frac{1}{m} \sum_{\alpha \beta}\left(\chi_{1234}^{d \alpha} \delta_{\alpha \beta}+G_{14}^{d \alpha} G_{23}^{d \beta}-G_{13}^{d \alpha} G_{24}^{d \beta}\right)
\end{aligned}
$$

is obtained. Next, the $f$-degrees of freedom are integrated out from eq. (4.81). One obtains

$$
\begin{aligned}
F[\bar{K}, K] & =\left\{\bar{K}_{1}\left[g\left(\Delta_{\omega}-\epsilon_{k}\right) g\right] K_{1}+\ln \int D[\bar{c}, c] \exp (-S[\bar{c}, c]\right. \\
& \left.\left.+\bar{K}_{1}\left[g\left(\Delta_{\omega}-\epsilon_{k}\right)\right] c_{1}+\bar{c}_{1}\left[\left(\Delta_{\omega}-\epsilon_{k}\right) g\right] K_{1}\right)\right\} .
\end{aligned}
$$

by completing the square

$$
\begin{aligned}
& -\frac{\lambda^{2}}{A^{2}} \bar{f} f-\lambda \bar{c} f-\lambda \bar{f} c+\bar{K} f+\bar{f} K \\
& =-\overline{\left(\frac{\lambda}{A} f+A \bar{c}-\frac{A}{\lambda} K\right)}\left(\frac{\lambda}{A} f+A \bar{c}-\frac{A}{\lambda} K\right)+A^{2} \bar{c} c+\frac{A^{2}}{\lambda^{2}} \bar{K} K-\frac{A^{2}}{\lambda} \bar{K} c-\frac{A^{2}}{\lambda} \bar{c} K .
\end{aligned}
$$

The same derivatives as in eqs. 4.84 and 4.85 are performed to obtain $G^{d}$ and $\chi_{1234}^{d}+G_{14}^{d} G_{23}^{d}-G_{13}^{d} G_{24}^{d}$ in terms of the $c$-degrees of freedom. For the dual Green function the result is

$$
\begin{aligned}
-\left.\frac{\delta^{2} F[\bar{K}, K]}{\delta K_{2} \delta \bar{K}_{1}}\right|_{\bar{K}_{1}=K_{2}=0} & =\left\{-g_{\omega}\left(\Delta_{\omega}-\epsilon_{k}\right) g_{\omega}\right. \\
& -\left.\frac{1}{\exp (F)}\right|_{\bar{K}_{1}=K_{2}=0} \int D[\bar{c}, c] \exp (-S[\bar{c}, c]) \times \\
& \times\left[g_{\omega}\left(\Delta_{\omega}-\epsilon_{k}\right) c_{2} \bar{c}_{1}\left(\Delta_{\omega}-\epsilon_{k}\right) g_{\omega}\right]
\end{aligned}
$$




$$
\begin{aligned}
& +\left(\left.\frac{1}{\exp (F)}\right|_{\bar{K}_{1}=K_{2}=0}\right)^{2} \int D[\bar{c}, c] \exp (-S[\bar{c}, c]) \times \\
& \left.\quad \times c_{2} \int D[\bar{c}, c] \exp \left(-S[\bar{c}, c] \bar{c}_{1}\right)\right\} \\
& =-g_{\omega}\left(\Delta_{\omega}-\epsilon_{k}\right) g_{\omega}+g_{\omega}\left(\Delta_{\omega}-\epsilon_{k}\right)\left\{G^{v}\right\}\left(\Delta_{\omega}-\epsilon_{k}\right) g_{\omega}=G^{d} .
\end{aligned}
$$

For $\chi_{1234}^{d}+G_{14}^{d} G_{23}^{d}-G_{13}^{d} G_{24}^{d}$ it follows that

$$
\begin{aligned}
\left.\frac{\delta^{4} F}{\delta K_{4} \delta K_{3} \bar{\delta} K_{2} \bar{K}_{1}}\right|_{K=0}= & \left.\frac{\delta^{4}}{\delta K_{4} \delta K_{3} \bar{\delta} K_{2} \bar{K}_{1}}\right|_{K=0} \frac{1}{m} \int D\left[\bar{c}^{\mu}, c^{\mu}\right] \exp \left(-S\left[\bar{c}^{\mu}, c^{\mu}\right]+\right. \\
& \left.+\bar{K}_{1}\left[g\left(\Delta_{\omega}-\epsilon_{k}\right)\right] c_{2}^{\mu}+\bar{c}_{1}^{\mu}\left[\left(\Delta_{\omega}-\epsilon_{k}\right) g\right] K_{2}\right)\left.\right|_{K=0} \\
= & \frac{\delta^{3}}{\delta K_{4} \delta K_{3} \bar{\delta} K_{2}} \frac{1}{m} \int D[\bar{c}, c]\left[g\left(\Delta_{\omega}-\epsilon_{k}\right)\right] c_{2}^{\mu} \exp \left(-S\left[\bar{c}^{\mu}, c^{\mu}\right]+\right. \\
& \left.+\bar{K}_{1}\left[g\left(\Delta_{\omega}-\epsilon_{k}\right)\right] c_{2}^{\mu}+\bar{c}_{1}^{\mu}\left[\left(\Delta_{\omega}-\epsilon_{k}\right) g\right] K_{2}\right)\left.\right|_{K=0} \\
= & {\left[g\left(\Delta_{\omega}-\epsilon_{k}\right)\right]\left[g\left(\Delta_{\omega}-\epsilon_{k}\right)\right]\left[\left(\Delta_{\omega}-\epsilon_{k}\right) g\right]\left[\left(\Delta_{\omega}-\epsilon_{k}\right) g\right] \times } \\
& \times \lim _{m \rightarrow 0} \frac{1}{m} \sum_{\alpha \beta \gamma \delta} \int D\left[\bar{c}^{\mu}, c^{\mu}\right] c_{1}^{\alpha} c_{2}^{\beta} \bar{c}_{3}^{\gamma} \bar{c}_{4}^{\delta} \exp \left(-S\left[\bar{c}^{\mu}, c^{\mu}\right]\right) \\
= & {\left[g\left(\Delta_{\omega}-\epsilon_{k}\right)\right]\left[g\left(\Delta_{\omega}-\epsilon_{k}\right)\right]\left[\left(\Delta_{\omega}-\epsilon_{k}\right) g\right]\left[\left(\Delta_{\omega}-\epsilon_{k}\right) g\right] \times } \\
& \lim _{m \rightarrow 0} \frac{1}{m} \sum_{\alpha \beta}\left(\chi_{1234}^{\alpha} \delta_{\alpha \beta}+G_{14}^{\alpha} G_{23}^{\beta}-G_{13}^{\alpha} G_{24}^{\beta}\right) .
\end{aligned}
$$

Equations 4.84 and 4.90 combined give

$$
G(\omega, k)=\left(\Delta_{\omega}-\epsilon_{k}\right)^{-1}+\frac{G^{d}}{g_{\mathrm{imp}}^{2}\left(\Delta_{\omega}-\epsilon_{k}\right)}
$$

for the single particle real lattice Green function. Combining eqs. 4.85) and 4.91 yields

$$
\begin{aligned}
\chi_{1234}^{\alpha} \delta_{\alpha \beta}+G_{14}^{\alpha} G_{23}^{\beta}-G_{13}^{\alpha} G_{24}^{\beta}= & \frac{G_{11}^{d \alpha}}{\left(g_{1}\left(\Delta_{1}-\epsilon_{1}\right) G_{11}^{\alpha}\right)} \frac{G_{22}^{d \beta}}{\left(g_{1}\left(\Delta_{1}-\epsilon_{1}\right) G^{\beta}\right)} \times \\
& \times\left(\chi_{1234}^{d \alpha} \delta_{\alpha \beta}+G_{14}^{d \alpha} G_{23}^{d \beta}-G_{13}^{d \alpha} G_{24}^{d \beta}\right) \times \\
& \times \frac{G_{33}^{d \beta}}{\left(g_{3}\left(\Delta_{3}-\epsilon_{3}\right) G_{33}^{\beta}\right)} \frac{G_{44}^{d \alpha}}{\left(g_{4}\left(\Delta_{4}-\epsilon_{4}\right) G_{44}^{\alpha}\right)}
\end{aligned}
$$

for the two-particle vertex. In eq. 4.93 replica indices are kept because the vertex 
is used to construct diagrams. If the replica limit is performed at the level of the vertex all terms that contain only disorder are removed. With that vertex one would miss diagrams that are important for the vertex corrections of the conductivity in section 4.5 ,

Dual and real fermion quantities are related by linear relations. These relations show that excitations of the real and dual system are the same [105], on the singleparticle level as well as for many-particle excitations.

\subsection{Self-Consistency in the Dual Fermion Approach}

Two levels of self-consistency exist in the dual fermion approach, which are defined by the solution of two problems, namely the impurity problem and the dual fermion problem. The expansion of the partition function for the dual fermions is written in terms of skeleton diagrams. To include further diagrams, the dual perturbation theory is solved self-consistently. Additionally, the hybridization function $\Delta_{\omega}$ has not yet been specified. A second loop analogous to the DMFT self-consistency is used to determine $\Delta_{\omega}$. As is shown in [105], an analogous choice of the hybridization function for the dual fermion approach is

$$
\Delta_{\omega}^{\text {new }}=\Delta_{\omega}^{\text {old }}+\xi\left[g_{\omega}^{-1} G_{\omega}^{d, \text { loc }}\left(G_{\omega}^{\text {loc }}\right)^{-1}\right]
$$

where $\xi \in[0,1]$ controls the mixing between the old and new hybridization function. The solution is self-consistent if $G^{d, l o c} \equiv 0$. This means that all diagrams containing a closed dual loop are summed at the level of the impurity problem, independently of their topology.

Calculating local quantities is equivalent to coarse-graining. Thus, it is at this point where a certain mean-field character is introduced in the dual fermion approach. Let us stress that this is only because the dual problem is not solved exactly. Figure 4.13 shows the self-consistency loop, which goes as follows:

0. Initialize the simulation with an impurity self-energy and an impurity vertex function, e. g. from DMFT. Go to step 2 .

1. Solve the impurity problem and calculate the impurity self-energy and impurity vertex function.

2. Parametrize the dual fermion calculation with the impurity self-energy and vertex function. 


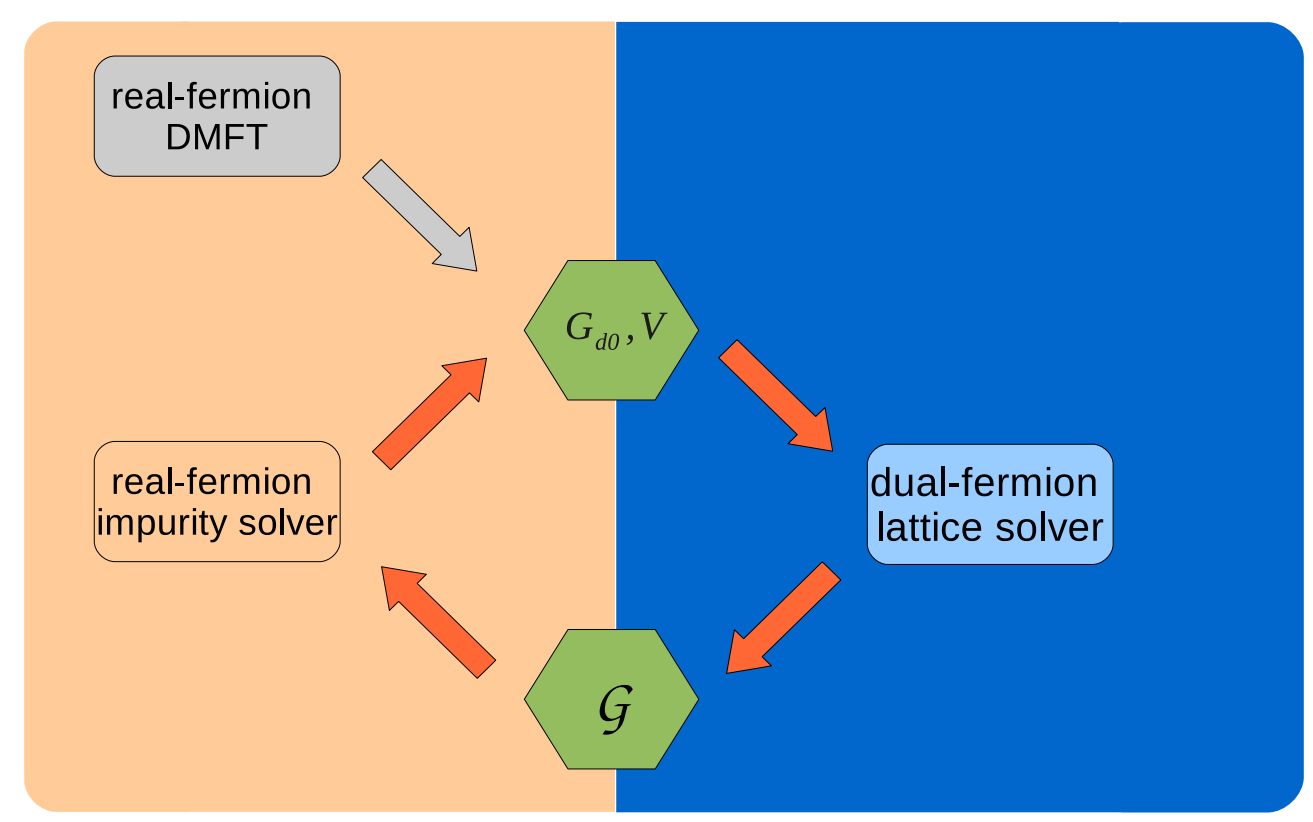

Figure 4.13: Self-consistency loop for the dual fermions. First, the dual fermion selfconsistency loop is initialized, e.g. with the DMFT solution. Second, the dual fermion problem is solved and defines a new impurity problem. Third, the impurity problem is solved and the output is used as input for the dual fermion problem. This is iterated until self-consistency is reached. (Based on illustration by S.-X. Yang [106]) 
3. Solve the dual fermion problem using self-consistent perturbation theory.

4. Calculate the new hybridization function and check for self-consistency.

5. If no self-consistent solution is reached, start over with step 1. Otherwise: STOP.

It should be remarked that the self-consistency loop presented here does not guarantee that one finds the optimal solution. The self-consistency condition given in eq. (4.94) is derived heuristically. In practice it turns out to give good results but a priori it is not clear that it sums relevant diagrams or is biased in some way.

This concludes the derivation of the self-consistent dual fermion approach. In the last section of this chapter we will show how the dual degrees of freedom can be used to construct an approximation for the vertex correction to the conductivity. 


\subsection{Observable of Interest: Conductivity}

Vertex corrections to the conductivity vanish in DMFT [109] because the vertex ladder has no momentum dependence. Dual fermions retrieve momentum dependence and thus vertex corrections remain finite and can be taken into account by means of the parquet equations [110]. According to [106, 111] the dc conductivity can be approximated as

$$
\sigma_{d c}=\frac{\beta^{2}}{\pi} \chi_{x x}\left(q=0, \tau=\frac{\beta}{2}\right) .
$$

$\chi_{x x}=\left\langle j_{x}(q, \tau) j_{x}(-q, 0)\right\rangle$ is the current-current correlation function. In the derivation it was assumed that $\operatorname{Im} \chi_{x x} \approx \omega \sigma_{d c}$ holds for an energy range $\Omega$ much larger than the temperature $T\left[111\right.$. This assumption does not hold for Fermi liquids as $\Omega \propto T^{2}$ at low temperatures. Hence, for small values of $U$ and $V$ in the Anderson-Hubbard model, eq. (4.95) is not a good description of the conductivity. Fortunately, we are interested in the conductivity around metal-insulator transitions, i.e. not in the Fermi liquid regime.

$\chi_{x x}$ is related to its Fourier transform in frequency space via

$$
\chi_{x x}\left(q=0, \tau=\frac{\beta}{2}\right)=T \sum_{i \nu_{m}} e^{-i \nu_{m} \frac{\beta}{2}} \chi_{x x}\left(q=0, i \nu_{m}\right) .
$$

The current operator for the $q=0$ component reads [70]

$$
J(q=0)=\frac{T}{N} \sum_{k \sigma} v_{k} c_{k \sigma}^{\dagger} c_{k \sigma},
$$

where $v_{k}=\frac{d}{d k} \epsilon(k) \cdot \chi_{x x}\left(q=0, i \nu_{m}\right)$ can be calculated as

$$
\begin{aligned}
\chi_{x x}\left(q=0, i \nu_{m}\right)= & \frac{T^{2}}{N^{2}} \sum_{\omega, \omega^{\prime} ; k, k^{\prime}} \chi_{x x}\left(q=0, i \nu_{m}\right)_{\omega, \omega^{\prime} ; k, k^{\prime}} \\
= & -\frac{T}{N} \sum_{\omega ; k} v_{k}^{2} \chi_{0}\left(q=0, i \nu_{m}\right)_{\omega ; k} \\
& -\frac{T^{2}}{N^{2}} \sum_{\omega, \omega^{\prime} ; k, k^{\prime}} v_{k} \chi_{0}\left(q=0, i \nu_{m}\right)_{\omega ; k} \times \\
& \times F^{r f}\left(q=0, i \nu_{m}\right)_{\omega, \omega^{\prime} ; k, k^{\prime}} \chi_{0}\left(q=0, i \nu_{m}\right)_{\omega^{\prime} ; k^{\prime}} v_{k^{\prime}} .
\end{aligned}
$$

Here, $F^{r f}$ is the real fermion vertex which can be calculated from the dual fermion vertex $F^{d}$ via

$$
F^{r f}(q)_{p p^{\prime}}=T(p+q) T(p) F^{d} T\left(p^{\prime}+q\right) T\left(p^{\prime}\right)
$$


with

$$
T(p)=-\frac{1}{1+g(p) \Sigma^{d}(p)} .
$$

The transformation formula follows from eq. (4.93) and

$$
G^{-1}(\Delta-\epsilon)^{-1} g^{-1} G^{d}=-\left(1+\Sigma^{d} g\right) .
$$

The last equality is shown in [105]. $F^{d}$ can be approximated by the vertex ladder. From the calculation of the self-energy the construction for the second order and FLEX approximations are known, but several changes have to be made compared to the self-energy. The external Green functions impose different constraints for the conductivity than for the self-energy. For example,

$$
J \propto \sum_{\sigma} c_{\sigma}^{\dagger} c_{\sigma}
$$

allows only for contributions where the in- and out-going Green functions on each side of the vertex ladder carry the same spin index. Also, a chain only consisting of disorder vertices is physical here, since the replica indices are now all fixed due to the in and out-going Green functions. The corresponding diagrams are created when the bubble diagram is disorder-averaged. The bubble diagram is obtained by contracting the ends of the unconnected horizontal two-particle Green function, and it is given by the contribution in the first line of eq. (4.99).

\subsubsection{Horizontal Channel}

In first order, the bare vertex is used. There are two possible spin configurations. The final result for the first order contribution is

$$
\Phi_{1 s t}=\frac{1}{2}\left(\tilde{V}_{d}+\tilde{V}_{m}\right)+\frac{1}{2}\left(\tilde{V}_{d}-\tilde{V}_{m}\right)=\tilde{V}_{d}
$$

Inserting this contribution in eq. 4.99 yields zero for the vertex corrections as there is no momentum dependence. For a momentum-independent vertex function the vertex correction is zero.

Four spin configurations are possible for the particle-hole channel. They are depicted in fig. 4.14. The vertex function $F$, and thus the dual potential, has three different contributions, the interaction vertex, the disorder vertex and the rotated disorder vertex. They have to be combined such that only allowed diagrams are constructed. Figures 4.15 to 4.18 show all possible spin configurations with the nine possible combinations of vertex functions. Unphysical diagrams are crossed out. The bottom right diagram is removed due to the replica limit in all cases.

The rotated disorder vertex is special for the particle-hole horizontal channel. Before the disorder-average is performed the corresponding diagrams decay into two 

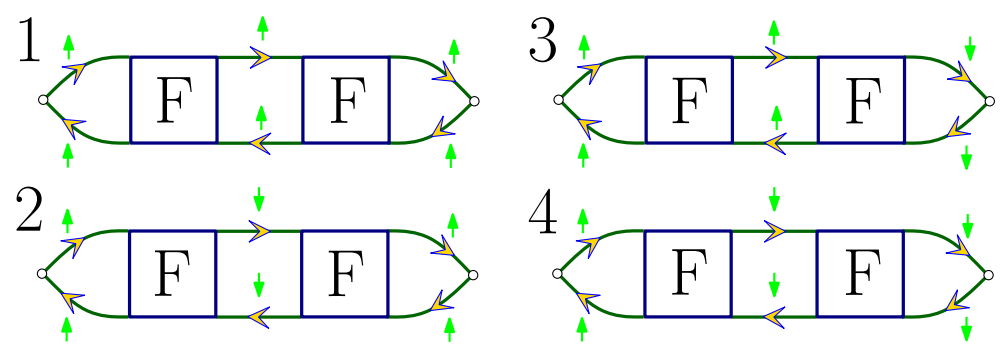

Figure 4.14: Possible spin configurations for the particle-hole horizontal channel.

parts which represent expectation values of the current operator. Without external fields these expectation values are zero and thus the corresponding diagrams cannot be present after the disorder-average. All other diagrams are removed because the spin configurations are not possible for the given combination of vertices.

The diagrams shown in figs. 4.15 to 4.18 are diagrams for the conductivity. Diagrams for the vertex ladder are obtained by removing the outer four Green functions. First, diagram fig. 4.15 translates to:

$$
\begin{aligned}
\Phi_{1}^{p h}=\frac{1}{2}\left(V_{d}+V_{m}\right) \bar{\chi}_{0}^{p h} & \frac{1}{2}\left(V_{d}+V_{m}\right)+\frac{1}{2}\left(V_{d}+V_{m}\right) \bar{\chi}_{0}^{p h} V_{0} \\
& +V_{0} \bar{\chi}_{0}^{p h} \frac{1}{2}\left(V_{d}+V_{m}\right)+V_{0} \bar{\chi}_{0}^{p h} V_{0} .
\end{aligned}
$$

For fig. 4.16 one gets

$$
\Phi_{2}^{p h}=\frac{1}{2}\left(V_{d}-V_{m}\right) \bar{\chi}_{0}^{p h} \frac{1}{2}\left(V_{d}-V_{m}\right) .
$$

For fig. 4.17 one obtains

$$
\Phi_{3}^{p h}=\frac{1}{2}\left(V_{d}+V_{m}\right) \bar{\chi}_{0}^{p h} \frac{1}{2}\left(V_{d}-V_{m}\right)+V_{0} \bar{\chi}_{0}^{p h} \frac{1}{2}\left(V_{d}-V_{m}\right) .
$$

Finally, for fig. 4.18 one gets

$$
\Phi_{4}^{p h}=\frac{1}{2}\left(V_{d}-V_{m}\right) \bar{\chi}_{0}^{p h} \frac{1}{2}\left(V_{d}+V_{m}\right)+\frac{1}{2}\left(V_{d}-V_{m}\right) \bar{\chi}_{0}^{p h} V_{0} .
$$

Defining

$$
\tilde{V}_{d}=V_{d}+V_{0}
$$

the particle-hole vertex ladder can be written as

$$
\Phi_{2 n d}^{p h=}=\Phi_{1}^{p h}+\Phi_{2}^{p h}+\Phi_{3}^{p h}+\Phi_{4}^{p h}=\tilde{V}_{d} \bar{\chi}_{0}^{p h} \tilde{V}_{d} .
$$



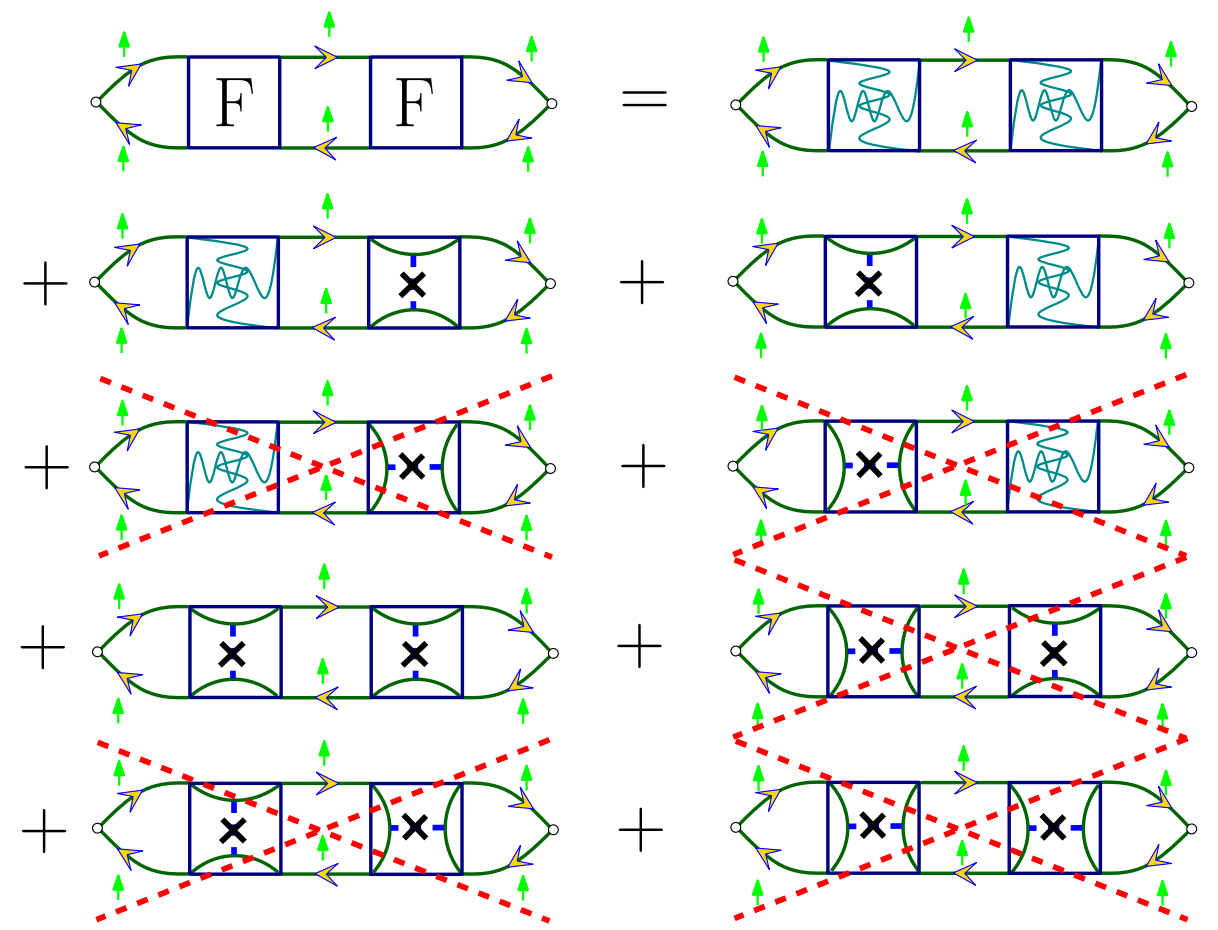

Figure 4.15: There are nine possible diagrams for the second order contribution to the conductivity. Above, the $(\uparrow \uparrow) \rightarrow(\uparrow \uparrow) \rightarrow(\uparrow \uparrow)$ contribution is analyzed. For all possible spin configuration, the bottom right diagram is removed by the replica limit. Additionally, all diagrams with the vertical disorder vertex vanish, because they are proportional to $\langle\vec{j}\rangle=0$ before the disorder average. 

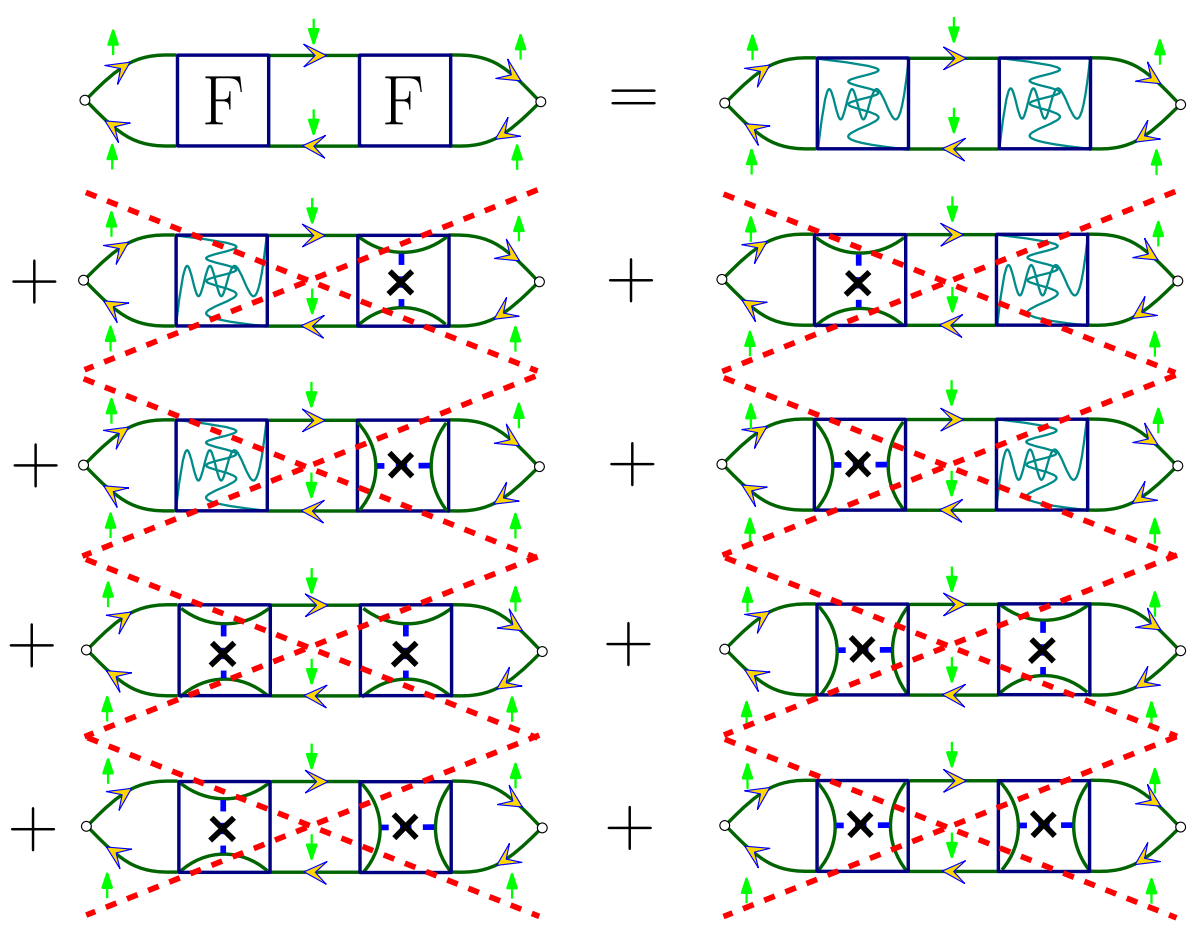

Figure 4.16: For the $(\uparrow \uparrow) \rightarrow(\downarrow \downarrow) \rightarrow(\uparrow \uparrow)$ contribution only the upper right diagram is allowed. This is because all interactions conserve spin.

This can be continued for higher order terms and one can derive

$$
\Phi_{F L E X}^{p h=}=\frac{\tilde{V}_{d}}{1-\tilde{V}_{d} \bar{\chi}_{0}^{p h}}
$$

for the FLEX approximation. The horizontal channel has no momentum dependence, therefore it does not contribute to the vertex corrections. It has been presented in this much detail for instructional purposes. 


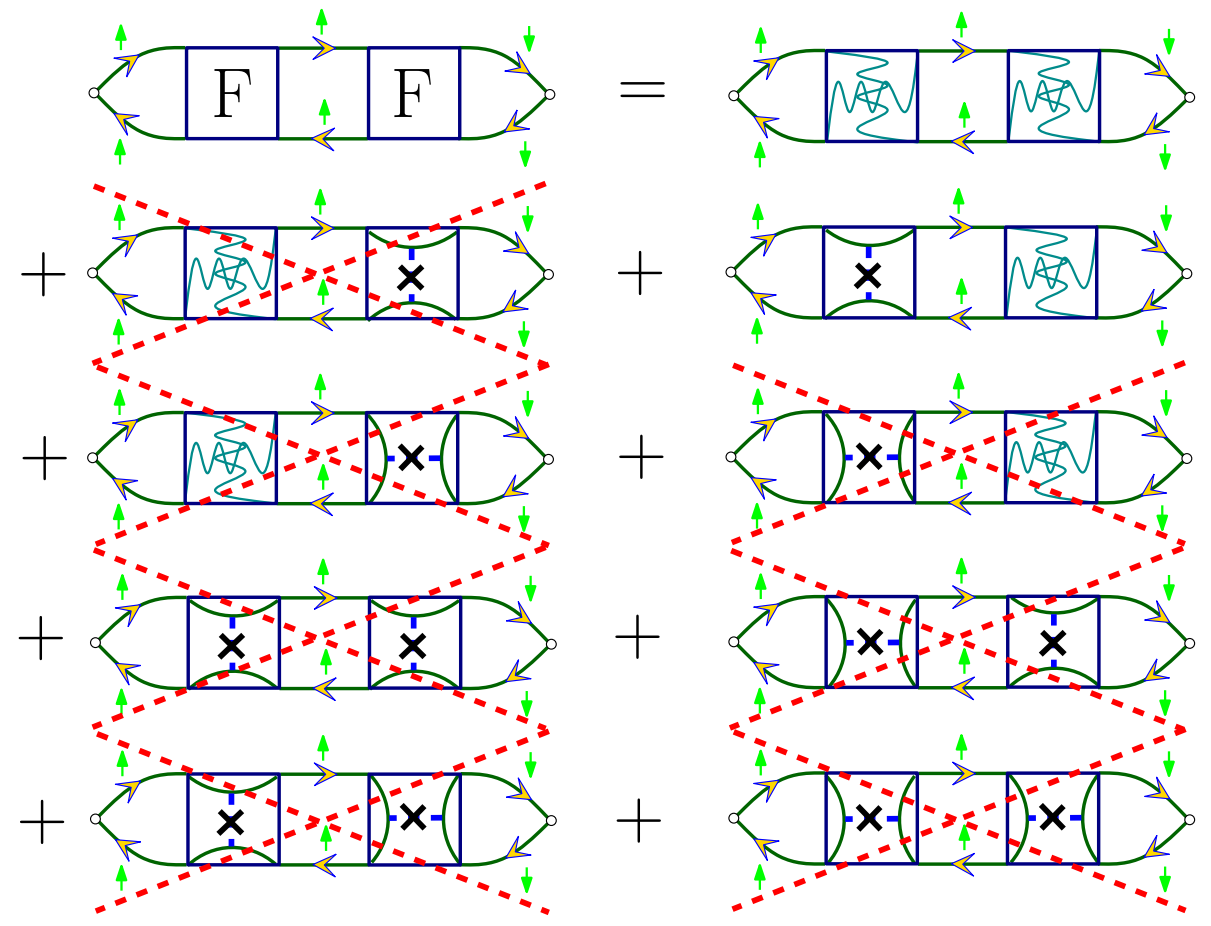

Figure 4.17: For the $(\uparrow \uparrow) \rightarrow(\uparrow \uparrow) \rightarrow(\downarrow \downarrow)$ contribution two diagrams are possible. The difference between the two diagrams on the second line is that on the left the disorder vertex connects different spins, which is not allowed, whereas on the right it connects the same spin. 


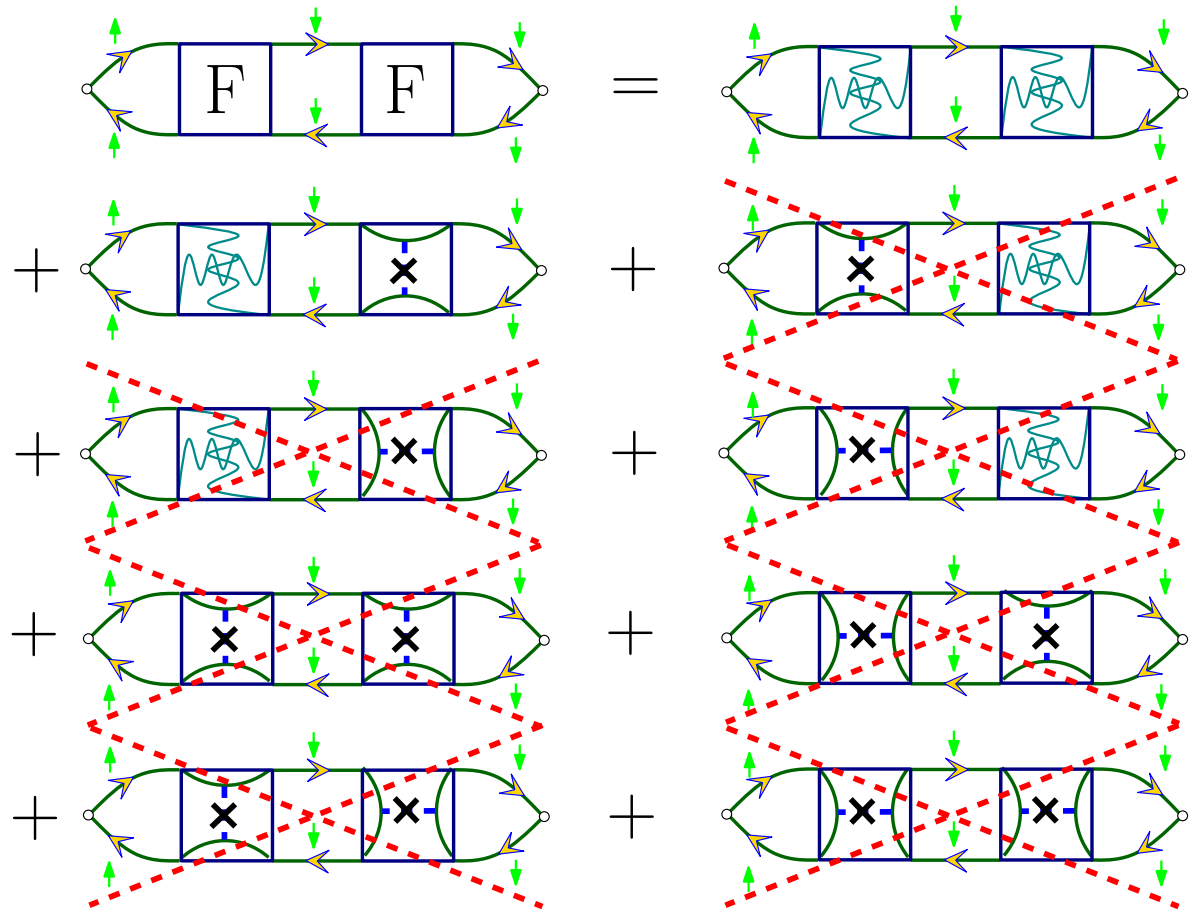

Figure 4.18: For the $(\uparrow \uparrow) \rightarrow(\downarrow \downarrow) \rightarrow(\downarrow \downarrow)$ contribution two diagrams are possible. The first diagram on the second line is possible because the disorder vertex connects the same spin. In the second diagram the disorder vertex connects different spins and thus the diagram is not possible. 


\subsubsection{Vertical Channel}

The contributions to the particle-hole vertical channel can be calculated by rotating the vertical vertex ladder to the horizontal one. This is illustrated in fig. 4.19. It follows

$$
\Phi_{c}^{p h \|}(q=0 ; \nu)_{\omega, \omega^{\prime} ; k, k^{\prime}}=-\Phi_{c}^{p h=}\left(k^{\prime}-k ; \omega^{\prime}-\omega\right)_{\omega, \omega+\nu ; k, k}
$$

where the index $c$ represents the channel and the approximation (2nd order, FLEX). There are three allowed spin configurations for the second order. These are shown in fig. 4.20. Note that the replica limit removes all diagrams with more than one disorder vertex in the vertical channel. Unlike in the horizontal case, the rotated disorder vertex can appear now, though only once. Otherwise, there would be a closed Fermi loop and the diagram would be canceled by the replica limit.

For diagram 1, one obtains

$$
\begin{aligned}
& \Phi_{1}^{p h \|}=\frac{1}{2}\left(V_{d}+V_{m}\right) \bar{\chi}_{0}^{p h} \frac{1}{2}\left(V_{d}+V_{m}\right)+\frac{1}{2}\left(V_{d}+V_{m}\right) \bar{\chi}_{0}^{p h} V_{0}+V_{0} \bar{\chi}_{0}^{p h} \frac{1}{2}\left(V_{d}+V_{m}\right) \\
& +\frac{1}{2}\left(V_{d}+V_{m}\right) \bar{\chi}_{0}^{p h} V_{0}^{\|}+V_{0}^{\|} \bar{\chi}_{0}^{p h} \frac{1}{2}\left(V_{d}+V_{m}\right)+V_{0} \bar{\chi}_{0}^{p h} V_{0}+V_{0} \bar{\chi}_{0}^{p h} V_{0}^{\|}+V_{0}^{\|} \bar{\chi}_{0}^{p h} V_{0} .
\end{aligned}
$$

Diagram 2 gives

$$
\Phi_{2}^{p h \|}=V_{m} \bar{\chi}_{0}^{p h} V_{m}+V_{m} \bar{\chi}_{0}^{p h} V_{0}+V_{0} \bar{\chi}_{0}^{p h} V_{m}+V_{0} \bar{\chi}_{0}^{p h} V_{0}
$$

and diagram 3

$$
\Phi_{3}^{p h \|}=\frac{1}{2}\left(V_{d}-V_{m}\right) \bar{\chi}_{0}^{p h} \frac{1}{2}\left(V_{d}-V_{m}\right)+\frac{1}{2}\left(V_{d}-V_{m}\right) \bar{\chi}_{0}^{p h} V_{0}^{\|}+V_{0}^{\|} \bar{\chi}_{0}^{p h} \frac{1}{2}\left(V_{d}-V_{m}\right) .
$$

Overall, the vertex ladder reads

$$
\Phi_{2 n d}^{p h \|}=\frac{1}{2} V_{d}^{*} \bar{\chi}_{0}^{p h} V_{d}^{*}-2 V_{0}^{\|} \bar{\chi}_{0}^{p h} V_{0}^{\|}+\frac{3}{2} \tilde{V}_{m} \bar{\chi}_{0}^{p h} \tilde{V}_{m}-2 V_{0} \bar{\chi}_{0}^{p h} V_{0}
$$

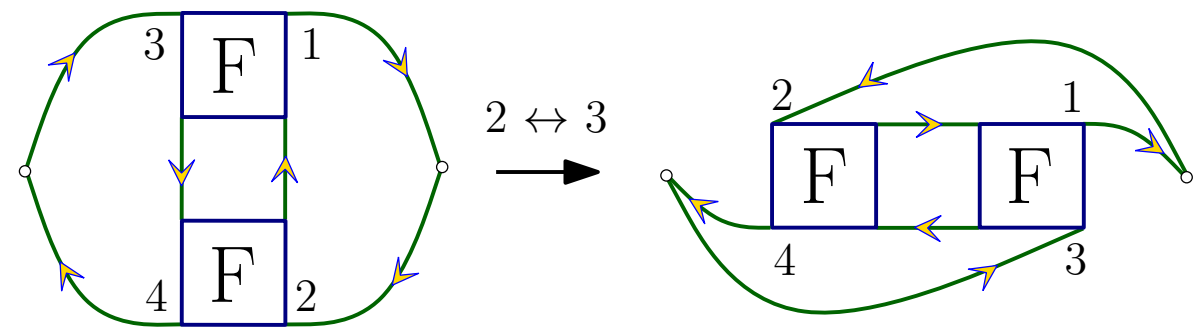

Figure 4.19: To calculate the contributions from the particle-hole vertical channel, the vertex is rotated to the particle-hole horizontal channel by exchanging $2 \leftrightarrow 3$. Note that this rotation comes with a minus sign. 

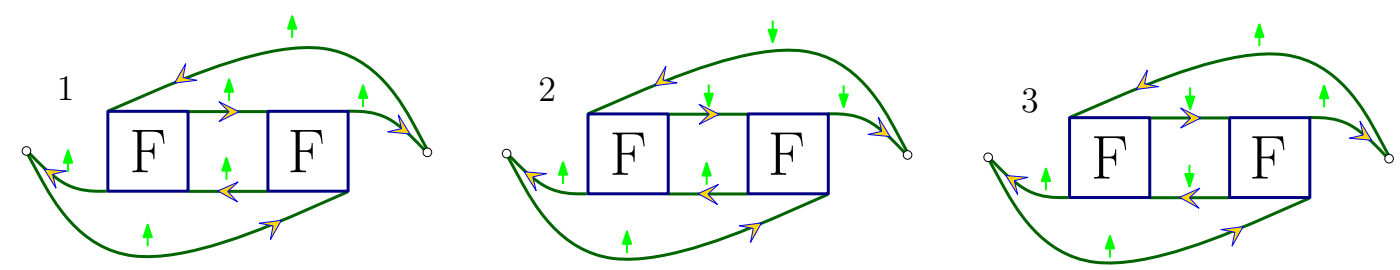

Figure 4.20: The possible spin configurations for the second order contributions.
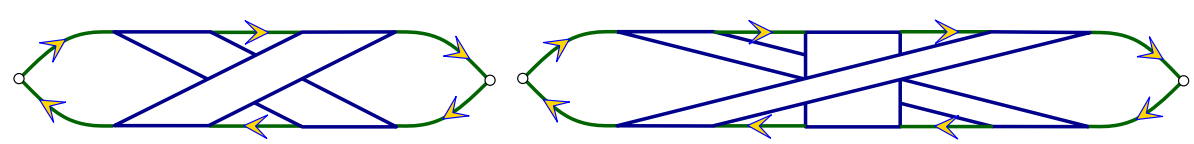

Figure 4.21: Maximally crossed diagrams in second and third order.

with

$$
V_{d}^{*}=V_{d}+V_{0}+2 V_{0}^{\|} \quad \tilde{V}_{m}=V_{m}+V_{0} .
$$

The pure disorder term is removed because left and right of the diagram are only connected via disorder and two replica indices remain. Including higher orders, the FLEX approximation is obtained as

$$
\Phi_{F L E X}^{p h \|}=\frac{1}{2} \frac{\tilde{V}_{d}}{1-\tilde{V}_{d} \bar{\chi}_{0}^{p h}}+\frac{3}{2} \frac{\tilde{V}_{m}}{1-\tilde{V}_{m} \bar{\chi}_{0}^{p h}}+\frac{1}{1-\tilde{V}_{d} \bar{\chi}_{0}^{p h}} V_{0}^{\|} \frac{1}{1-\bar{\chi}_{0}^{p h} \tilde{V}_{d}}-2 \frac{V_{0}}{1-\bar{\chi}_{0}^{p h} V_{0}} .
$$

\subsubsection{Maximally Crossed Channel}

A third class of diagrams can be included up to infinite order. These are the maximally crossed diagrams which are shown in fig. 4.21. These diagrams can be transformed such that they look like particle-particle diagrams internally. That way, they can be calculated from the particle-particle vertex, which is shown in fig. 4.22. The transformation leads to a total momentum of $k+k^{\prime}$ and total energy of $\omega+\omega^{\prime}+\nu$ for the vertex ladder

$$
\Phi_{c}^{p h \times}(q=0 ; \nu)_{\omega, \omega^{\prime} ; k, k^{\prime}}=-\Phi_{c}^{p p}\left(k+k^{\prime} ; \omega+\omega^{\prime}+\nu\right)_{-\omega^{\prime},-\omega ;-k^{\prime},-k} .
$$

For the particle-particle channel three spin configuration appear to be possible. These are shown in fig. 4.23. The first diagram carries a factor $\frac{1}{2}$ because the two internal lines are indistinguishable. It corresponds to the triplet channel.

It is useful to remember the definition

$$
\tilde{V}_{p}=V_{p}+V_{0}
$$




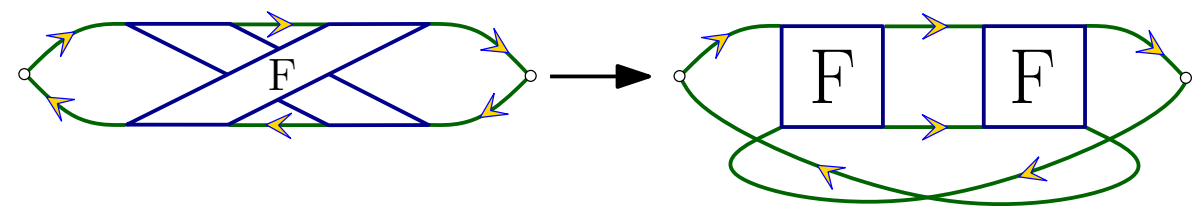

Figure 4.22: By exchanging the bottom left and bottom right corner of the vertex ladder, the maximally crossed diagram is transformed to a diagram that is internally a particle-particle diagram.

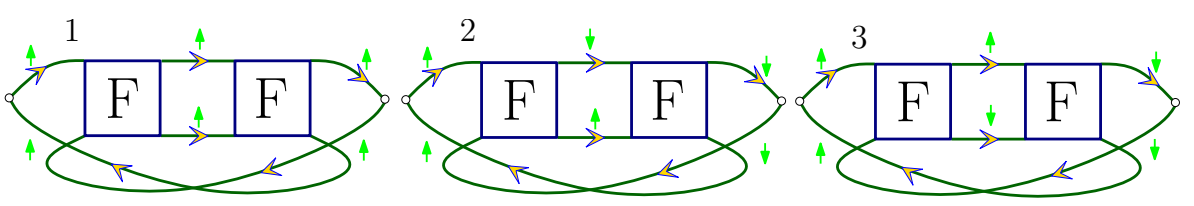

Figure 4.23: Spin configurations for the particle-particle channel. The first contribution carries a factor $\frac{1}{2}$ because the two internal lines are indistinguishable. The second and third diagrams are topologically equivalent.

and then write the triplet vertex as

$$
\tilde{V}_{t}=\tilde{V}_{p}+\tilde{V}_{p}^{\prime}
$$

Using $\tilde{V}_{p}$ it is easy to include the disorder contributions. $\tilde{V}_{t}$ is crossing symmetric, therefore one has to take into account only the first diagram in fig. 4.23.

For the first diagram one gets

$$
\Phi_{2 n d}^{t}=\tilde{V}_{p} \bar{\chi}_{0}^{p p} \tilde{V}_{p}+\tilde{V}_{p} \bar{\chi}_{0}^{p p} \tilde{V}_{p}^{\prime}-V_{0} \bar{\chi}_{0}^{p p} V_{0}^{\times}
$$

which is second order approximation. $V_{0} \bar{\chi}_{0}^{p p} V_{0}^{\times}$has to be subtracted because this diagram can be cut into the left and right part by cutting only disorder vertices. Thus, it is zero before the disorder-average.

Equation 4.122 can easily be generalized to higher orders and the FLEX approximation becomes

$$
\Phi_{F L E X}^{t}=\frac{1}{1-\tilde{V}_{p} \bar{\chi}_{0}^{p p}}\left(\tilde{V}_{p}+\tilde{V}_{p}^{\prime}\right)-\frac{V_{0}^{\times}}{1-V_{0} \bar{\chi}_{0}^{p p}} .
$$

Note that there is no particle-particle vertical channel. The corresponding contribution is already included in the horizontal particle-particle channel because of the crossing symmetry.

Finally, the dual vertex reads

$$
\begin{aligned}
F^{d}= & 2\left(\Phi_{c}^{p h=}(q=0 ; \nu)_{\omega, \omega^{\prime} ; k, k^{\prime}}-\Phi_{c}^{p h=}\left(k^{\prime}-k ; \omega^{\prime}-\omega\right)_{\omega, \omega+\nu ; k, k}\right. \\
& \left.-\Phi_{c}^{p p}\left(k+k^{\prime} ; \omega+\omega^{\prime}+\nu\right)_{-\omega^{\prime},-\omega ;-k^{\prime},-k}\right) .
\end{aligned}
$$


The factor of 2 comes from the fact that we ignored half of the spin configurations which are connected to the contributions above by spin symmetry.

The next step is to transform the dual vertex to the real fermion space. This is easily done using the transformation matrices for vertices ([105]A.136-138)

$$
F^{r f}(q)_{p, p^{\prime}}=T(p+q) T(p) F^{d}(q)_{p, p^{\prime}} T\left(p^{\prime}+q\right) T\left(p^{\prime}\right)
$$

where

$$
T=-\frac{1}{1+g \Sigma^{d}}
$$

This concludes the calculation of the conductivity. It is possible to apply the same reasoning for other quantities like the susceptibility. This is shown for the clean system in [112].

\subsection{Summary}

In this chapter we presented the dual fermion approach for disordered interacting systems, the main result of this thesis. We started with the derivation of the formalism, where we used the replica trick to replace $\{\ln Z\}$ by powers of the disorderaveraged partition function $\{Z\}$. After the replica trick, it was possible to integrate out the disorder in favor of an effective interaction between replicas. The dual degrees of freedom were introduced and the dual potential has been calculated. With the dual potential at our disposal, we were able to obtain a perturbative solution of the dual fermion lattice problem in terms of a self-consistent second order and FLEX approximation.

Unlike for the clean system [105], the replica limit imposes topological restrictions on the dual fermion diagrams. These restrictions remove dual diagrams that have a topology that is impossible for real fermion diagrams. This was already a hint at the strong relation between dual and real excitations.

Next, dual and real fermion quantities were related by exact linear relations, thus giving a prescription to obtain physical quantities from the dual degrees of freedom. The fact that these relations are linear, is interpreted as dual and real fermions having the same excitations.

To conclude the formalism, we wrote down the self-consistency loop for the dual fermion approach, which is analogous to the DMFT self-consistency loop. We use the self-consistency condition that the local dual Green function vanishes. Hence, all dual fermion diagrams with a local dual Green function are implicitly summed.

At the end, the exact relation for the vertex function was used to calculated vertex corrections for the conductivity. The conductivity will be used later on to determine the metal-insulator transition for strong disorder.

So far, we did not specify how to solve the impurity and cluster problems in sec- 
tions 3.1 and 3.2 and chapter 4 . In the next chapter, the continuous-time Quantum Monte Carlo method is introduced to tackle this task. 


\section{Quantum Monte Carlo for Impurity Problems}

One common aspect of all the methods presented in sections 3.1 and 3.2 and chapter 4 is the underlying impurity problem. Several methods exist for the solution of impurity problems. These methods are referred to as impurity solvers or just solvers. Which solver is used depends on the specifics of the problem at hand, i.e. calculations at $T=0$ or $T>0$, are two-particle quantities needed or how many orbitals or sites comprise the impurity?

The solver of choice for dynamical mean-field theory (DMFT) involving only oneparticle quantities is arguably the numerical renormalization group (NRG) [113, 114. It provides real frequency spectra and can be applied over a wide range of parameters. However, practical calculations are mostly limited to one orbital or one site, respectively. This eliminates NRG from the list of possible solvers for the dynamical cluster approximation (DCA). The access to two-particle quantities is very expensive which makes it a bad choice for the dual fermion approach, where two-particle quantities are needed with their full dependence on three independent frequencies.

An alternative method for impurity problems is exact Diagonalization (ED). ED in the context of DMFT and cluster extensions thereof is reviewed in [115]. As the name suggests, the Hamiltonian is diagonalized by means of numerical algorithms. The solution is exact at most up to machine precision, depending on the algorithm used. Popular examples are the Lanczos algorithm [116] and the Jacobi-Davidson algorithm [117]. Which ED algorithm is used depends on the problem, e.g. are there degeneracies that one wishes to resolve, is one only interested in the ground state or are excited states needed?

Despite its flexibility the applicability of ED is limited to small systems. The exponential growth of the Hilbert space restricts ED to a relatively small number of sites, e. g. $\approx 20$ for the square lattice Hubbard model around half-filling [118, 119]. A further drawback is the limitation to a discretized bath as the continuum of states of the infinite lattice is mapped to a finite cluster with discrete energy levels. This can lead to systematic errors. Therefore, one has to check that the self-energy converges as the number of bath sites is increased. If this convergence can be achieved, ED is a good choice for DMFT. Its applicability to dual fermions is less ideal, though. The calculation of vertex functions with the full dependence on three frequencies is 
quite expensive.

In [115] the number of possible cluster sites for cluster-extensions of DMFT is given as six. This small number comes from the fact that bath sites are needed. Even in 1d, this does not give a converged result in the number of cluster sites and hence ED is not suited for DCA in the context of this work.

Finally, a very popular method for $1 \mathrm{~d}$ systems is the density matrix renormalization group (DMRG) [2]. There are attempts to utilize it as an impurity solver [120, 121, 122] but it has not yet established itself as such. It is a promising candidate for DMFT, but its applicability to DCA, which is mainly used in $2 \mathrm{~d}$ and $3 \mathrm{~d}$ systems, has yet to be explored. DMRG is not suited for dual fermions because access to the two-particle Green function is numerically too costly.

Quantum Monte Carlo (QMC) can be readily applied for 1d, 2d and 3d systems as well as for a single impurity. Two particle quantities can be expensive to calculate, but still come at reasonable computational cost. An advantage of QMC is that it can be easily parallelized such that it can exploit high performance computing resources. Until the beginning of the 21st century, the standard method was HirschFye quantum Monte Carlo [123]. It suffers from a systematic error which is due to the discretized imaginary time variable, but this error can be removed by a systematic extrapolation $\Delta \tau \rightarrow 0$. Nowadays, new algorithms [42, 43, 124, 125, 25] allow to avoid the discretization of the time variable altogether and thereby avoid systematic errors. These methods are referred to as continuous-time quantum Monte Carlo (ctQMC). One major drawback of QMC is that efficient equilibrium algorithms work in imaginary time, whereas one desires dynamical quantities in real-time. This requires analytic continuation [126] if one wishes to look at quantities like the density of states. Real-time algorithms do exist [127, 128] but their usage is not advisable for equilibrium systems.

Its versatility and the high potential for parallelization make ctQMC the method of choice for this thesis. As the same ctQMC solver can be applied to the impurity problem in section 3.1 and chapter 4 and to the cluster problem in section 3.2 , it is better to adopt this single method and use a highly optimized code than to try different impurity solvers and find the best solver for each individual problem. Also, method development is the main scope of this thesis, such that a solver that can be easily adapted to handle more complex impurities and interactions is desirable and at present only ctQMC fulfills this requirement. In the remainder of this chapter the basic ideas of the interaction expansion ctQMC [42, 43, 44] are introduced.

\subsection{Basic Ideas of the Monte Carlo Method}

The starting point for ctQMC is the partition function

$$
Z=\operatorname{Tr} e^{-\beta H}
$$


In general, the trace cannot be evaluated exactly. The associated sums or integrals are usually high-dimensional. In our case we have a bath in the thermodynamic limit which means we have infinite dimensions. Integration ${ }^{1}$ routines sum contributions to an integral by employing a systematic grid to discretize a continuous system and approximating integrals by sums. This can become very inefficient for such a high-dimensional system. The scaling of the error is generally of the form $N^{-\frac{c}{d}}$ [129] where $N$ is the number of sample points, $c$ is a constant that depends on the algorithm and $d$ is the dimension of the integral.

A way out are Monte Carlo simulations [130]. In a Monte Carlo simulation sample points are chosen at random to approximate the integral. Using this method, a scaling of the error as $N^{-\frac{1}{2}}$ can be achieved. This scaling is independent of the dimension of the integral [131].

Monte Carlo methods require that a weight or probability is attached to every sample point. To this end, we rewrite the partition function as a sum

$$
Z=\sum_{C} w_{C}
$$

where $C$ denotes a configuration and $w_{C}$ the corresponding weight. "Configuration" is part of the Monte Carlo vocabulary and is just another name for a sample point. In principle, $w_{C}$ can be positive or negative, e.g. expanding the exponential $e^{-\beta H}$ explicitly introduces minus signs. As probabilities can only be greater or equal zero, so should the weights. Thus we have to modify eq. (5.2) such that we have positive weights only. We get

$$
Z=\sum_{C} \operatorname{sgn}\left(w_{C}\right)\left|w_{C}\right|
$$

Here, $\operatorname{sgn}\left(w_{C}\right)$ is the sign of $w_{C}$. Now $\left|w_{C}\right| \geq 0$ can be used as a weight for a configuration. Expectation values can be calculated according to

$$
\langle O\rangle \approx\langle O\rangle_{M C}=\frac{\sum_{i=1}^{N} O_{C_{i}} w_{C_{i}}}{\sum_{i=1}^{N} w_{C_{i}}}=\frac{\sum_{i=1}^{N} O_{C_{i}} \operatorname{sgn}\left(w_{C_{i}}\right)\left|w_{C_{i}}\right|}{\sum_{i=1}^{N} \operatorname{sgn}\left(w_{C_{i}}\right)\left|w_{C_{i}}\right|},
$$

where $N$ is the number of configurations, $\langle O\rangle_{M C}$ is a Monte Carlo estimate and $O_{i}$ denotes the expectation value of $O$ in the configuration $i$. Equation (5.4) follows from eqs. (5.1) and (5.3) by taking a logarithmic derivative with respect to some source field. Approximating the sum over all configurations by a sum over a finite number of random configurations yields the Monte Carlo estimate.

Using eq. (5.4) it is possible to calculate expectation values of observables, but in general it will not be efficient. Simply creating random configurations carries the risk of sampling unimportant contributions and missing the important ones, thereby requiring a very large number of configurations. To overcome this problem,

\footnotetext{
${ }^{1}$ Here and in the following "integration" includes summation as well.
} 
importance sampling [131] is used. Importance sampling creates configurations with a probability proportional to their weights. One benefit of importance sampling is that observables can be estimated as [132]

$$
\langle O\rangle_{M C} \approx \frac{1}{N} \sum_{i=1}^{N} O_{i}
$$

if the right scheme for creating configurations is employed. This scheme will be introduced in this section. The variance $\operatorname{Var}(O)$ is estimated according to

$$
\operatorname{Var}(O) \approx\left\langle O^{2}\right\rangle_{M C}-\langle O\rangle_{M C}^{2}
$$

Equation (5.6) is only valid if the $O_{i}$ are uncorrelated [133, otherwise eq. (5.6) will underestimate the variance.

Importance sampling generally improves the efficiency of Monte Carlo algorithms. The gain in efficiency depends on the problem at hand. For a constant function, every sample point has the same weight and is as important as all others. There would be no gain in efficiency if importance sampling is used. Trying to obtain uncorrelated data generally means one has to create additional configurations and as a result Monte Carlo procedures with importance sampling would even be less efficient than the algorithm without. If the integrand has non-zero contributions in only one percent of the sample space, only one percent of truly random sample points will contribute. Only those relevant sample points will be created if importance sampling is used. That way, the ratio of sample points to important sample points would be 100 times higher. If ten Monte Carlo steps are needed to obtain uncorrelated data the total gain in efficiency will be a factor of ten.

A random walk in configuration space is used to implement importance sampling. Starting from a configuration $C_{0}$, a chain of configurations

$$
C_{0} \rightarrow C_{1} \rightarrow C_{2} \rightarrow \cdots \rightarrow C_{n}
$$

is created. The probability for creating a certain configuration $C_{n}$ depends on configuration $C_{n-1}$ only, i. e. the random walk is memoryless. Such a random walk is called a Markov chain. Markov chains reduce memory usage as only one configuration has to be kept and it is not necessary to keep track of the trajectory of the random walk. For a large number of steps the Markov chain will approach a stationary distribution which is independent of the starting configuration. The probability distribution for a given random variable will become Gaussian in this limit. Besides these statistical properties that facilitate the evaluation, Markov chains are relatively easy to implement.

One way to create configurations according to their weights is to obey the following two conditions: 
- Ergodicity: In principle, any configuration $C_{A}$ must be reachable from any other configuration $C_{B}$ within a finite number of steps.

- Detailed Balance: The probability $\left|w_{C_{A}}\right| \cdot t\left(C_{A} \rightarrow C_{B}\right)$ of going from $C_{A}$ to $C_{B}$ must be equal to the probability $\left|w_{C_{B}}\right| \cdot t\left(C_{B} \rightarrow C_{A}\right)$ of going from $C_{B}$ to $C_{A}$.

This is achieved by employing a Metropolis-Hastings scheme [130, 132]. The transition probability $t$ is split into the proposal probability $p$ and the acceptance probability $a$

$$
t\left(C_{n} \rightarrow C_{n+1}\right)=p\left(C_{n} \rightarrow C_{n+1}\right) \cdot a\left(C_{n} \rightarrow C_{n+1}\right) .
$$

Combining this with the detailed balance condition yields

$$
\frac{a\left(C_{n} \rightarrow C_{n+1}\right)}{a\left(C_{n+1} \rightarrow C_{n}\right)}=\frac{\left|w_{C_{n+1}}\right| \cdot p\left(C_{n+1} \rightarrow C_{n}\right)}{\left|w_{C_{n}}\right| \cdot p\left(C_{n} \rightarrow C_{n+1}\right)}
$$

for the ratios of the acceptance probabilities. This equation is satisfied by

$$
a\left(C_{n} \rightarrow C_{n+1}\right)=\min \left(1, \frac{p\left(C_{n+1} \rightarrow C_{n}\right) \cdot\left|w_{C_{n+1}}\right|}{p\left(C_{n} \rightarrow C_{n+1}\right) \cdot\left|w_{C_{n}}\right|}\right) .
$$

This means that if the probability of accepting a move from $C_{n}$ to $C_{n+1}$ is greater than the probability of accepting a move from $C_{n+1}$ to $C_{n}$ we set it equal to one and accept the move. Using eq. (5.10) to determine the acceptance probability ensures a random walk with importance sampling.

\subsection{The Interaction Expansion}

The ideas and concepts outlined above can be applied in a variety of ways. There are the hybridization expansion [124], the interaction expansion [42, 43, 44] and a variation thereof, based on auxiliary fields [125]. We will use the interaction expansion. In this section, we provide some of the details of the random walk and the measurements of one- and two particle quantities.

\subsubsection{Monte Carlo Weights and the Random Walk}

For the derivation of the details of the ctQMC we follow [44]. First, we have to derive the configurational weights for the random walk. We start from the Hubbard Hamiltonian

$$
H_{H u b b}=\underbrace{-\sum_{i j, \sigma} t\left(c_{\sigma i}^{\dagger} c_{\sigma j}+\text { h.c. }\right)}_{H_{0}}+U \sum_{i}\left(n_{i \uparrow}-\frac{1}{2}\right)\left(n_{i \downarrow}-\frac{1}{2}\right) .
$$


The interaction term is modified according to

$$
\frac{U}{2} \sum_{i} \sum_{s= \pm 1}\left(n_{i \uparrow}-\frac{1}{2}-s \delta\right)\left(n_{i \downarrow}-\frac{1}{2}+s \delta\right),
$$

where $\delta$ is a parameter that can be adjusted to reduce the infamous sign problem (cf. appendix A). This modification does not change the Hamiltonian except for a constant, so the physics is not affected. In the next step the partition function is expanded as

$$
\begin{aligned}
\frac{Z}{Z_{0}}=\sum_{n=0}^{\infty}\left(\frac{-U}{2}\right)^{n} & \int_{0}^{\beta} d \tau_{1} \sum_{i_{1}, s_{1}} \ldots \int_{0}^{\tau_{n-1}} d \tau_{n} \times \\
& \times \sum_{i_{n}, s_{n}} \prod_{\sigma}\left\langle\left[n_{i_{1}, \sigma}\left(\tau_{1}\right)-\alpha_{\sigma}\left(s_{1}\right)\right] \ldots\left[n_{i_{n}, \sigma}\left(\tau_{n}\right)-\alpha_{\sigma}\left(s_{n}\right)\right]\right\rangle_{0}
\end{aligned}
$$

with $\alpha_{\sigma}(s)=\frac{1}{2}+\sigma s \delta$ and $\langle\ldots\rangle_{0}=\operatorname{Tr}\left[e^{-\beta H_{0}} \ldots\right] / Z_{0}$ and $Z_{0}=\operatorname{Tr}\left[e^{-\beta H_{0}}\right]$. Timeordering is encoded in the upper bounds of the integrals and removes the factor $\frac{1}{n !}$ which is usually associated with the expansion of an exponential. Note that we have $Z / Z_{0}$ instead of $Z$ as before. As we are interested in observables and not the partition function itself we have to calculate logarithmic derivatives and the factor $1 / Z_{0}$ is removed:

$$
\left.\frac{d}{d \eta} \ln \frac{Z[\eta]}{Z_{0}}\right|_{\eta=0}=\left.\frac{1}{Z} \frac{d Z[\eta]}{d \eta}\right|_{\eta=0}
$$

where $\eta$ is a source field. The one-particle Hamiltonian $H_{0}$ does not couple different spins, therefore the expectation value in eq. (5.13) decouples in spin up and spin down. This simplifies the evaluation of the expectation value as it is generally cheaper to calculate the determinants of two $n \times n$ matrices than to calculate one determinant of a $2 n \times 2 n$ matrix as the computational cost scales as $\mathbf{O}\left(n^{3}\right)^{2}$.

Next, we have to evaluate the expectation values $\langle\ldots\rangle_{0}$ for an individual configuration. As $H_{0}$ is only quadratic in $c$ and $c^{\dagger}$ this can be done by applying Wick's theorem [107] to eq. (5.13):

$$
\begin{aligned}
& \left\langle\left[n_{i_{1}, \sigma}\left(\tau_{1}\right)-\alpha_{\sigma}\left(s_{1}\right)\right] \ldots\left[n_{i_{n}, \sigma}\left(\tau_{n}\right)-\alpha_{\sigma}\left(s_{n}\right)\right]\right\rangle_{0}= \\
& \operatorname{det}\left(\begin{array}{cclc}
G_{i_{1}, i_{1}}^{0}\left(\tau_{1}, \tau_{1}\right)-\alpha_{\sigma}\left(b_{1}, s_{1}\right) & G_{i_{1}, i_{2}}^{0}\left(\tau_{1}, \tau_{2}\right) & \ldots & G_{i_{1}, i_{n}}^{0}\left(\tau_{1}, \tau_{n}\right) \\
G_{i_{2}, i_{1}}^{0}\left(\tau_{2}, \tau_{1}\right) & G_{i_{2}, i_{2}}^{0}\left(\tau_{2}, \tau_{2}\right)-\alpha_{\sigma}\left(b_{2}, s_{2}\right) & \ldots & G_{i_{2}, i_{n}}^{0}\left(\tau_{2}, \tau_{n}\right) \\
\vdots & \vdots & \ddots & \vdots \\
G_{i_{n}, i_{1}}^{0}\left(\tau_{n}, \tau_{1}\right) & G_{i_{n}, i_{2}}^{0}\left(\tau_{n}, \tau_{2}\right) & \ldots & G_{i_{n}, i_{n}}^{0}\left(\tau_{n}, \tau_{n}\right)-\alpha_{\sigma}\left(b_{n}, s_{n}\right)
\end{array}\right),
\end{aligned}
$$

\footnotetext{
${ }^{2}$ It is possible to calculate determinants using fast matrix multiplication and thereby achieving a complexity of $n^{2.373}[134$
} 
where the non-interacting Green function is

$$
G_{i j}^{0}\left(\tau_{1}, \tau_{2}\right)=-\left\langle T_{\tau} c_{i}\left(\tau_{1}\right) c_{j}^{\dagger}\left(\tau_{2}\right)\right\rangle_{0}
$$

It is assumed to be spin-independent. If necessary, it can be easily generalized to be spin-dependent, as the one-particle Hamiltonian does not couple different spins.

We can rewrite eq. (5.13) as a sum over weights using the definition

$$
\sum_{C_{n}}=\sum_{n=0}^{\infty} \int_{0}^{\beta} d \tau_{1} \sum_{i_{1}, s_{1}} \ldots \int_{0} d \tau_{n} \sum_{i_{n}, s_{n}} .
$$

A configuration is a set of tuples

$$
C_{n}=\left\{\left[i_{1}, \tau_{1}, s_{1}\right] \ldots\left[i_{n}, \tau_{n}, s_{n}\right]\right\} .
$$

The partition functions then becomes

$$
\frac{Z}{Z_{0}}=\sum_{C_{n}}\left(-\frac{U}{2}\right)^{n} \prod_{\sigma} \operatorname{det} M_{\sigma}\left(C_{n}\right),
$$

where $M_{\sigma}$ is the matrix on the right-hand side of eq. (5.15). The weight of a configuration for the random walk is

$$
\left|w_{C_{n}}\right|=\left|\left(-\frac{U}{2}\right)^{n} \prod_{\sigma} \operatorname{det} M_{\sigma}\left(C_{n}\right) d \tau^{n}\right|
$$

where the infinitesimals from the integrals are included in the weight. For simplicity, addition and removal of a vertex are suggested with equal probability. This gives a factor of $\frac{1}{2}$ for the addition and removal probability and thus cancels out in eq. 5.10). Every vertex has three components that have to be chosen at random. Without prior knowledge about the importance of configurations, it is advisable to attach equal probability to the possible choices for spin up and spin down, the choice of cluster site and the imaginary time $\tau$. Choosing a spin thus results in a factor $\frac{1}{2}$, a factor of $\frac{1}{N_{c}}$ comes from the choice of one out of $N_{c}$ equally likely cluster sites, and a factor $\frac{d \tau}{\beta}$ from picking a random time $\tau$ in the interval $[0, \beta)$. The probability for proposing the addition of a certain vertex is therefore

$$
P(n \rightarrow n+1)=\frac{d \tau}{2 \beta N_{c}} .
$$

The proposal probability of removing a given vertex is just inversely proportional 
to the number of vertices $N_{v}$, therefore

$$
P(n+1 \rightarrow n)=\frac{1}{N_{v}} .
$$

At this point, the weights $\left|w_{C_{n}}\right|$ and the probability $P(n \rightarrow n+1)$ contain infinitesimal factors of $d \tau$. Careful examination of eq. (5.10) shows that these factors cancel in the acceptance probability: In the numerator $P(n+1 \rightarrow n)$ contributes no factor, $\left|w_{C_{n+1}}\right|$ contributes $n+1$ factors. In the denominator $P(n \rightarrow n+1)$ contributes one factor and $\left|w_{C_{n}}\right|$ contributes $n$ factors, such that we have a total of $n+1$ factors as well, and thus the infinitesimals cancel.

\subsubsection{Single-Particle Measurements}

According to eq. (5.4), the Monte Carlo estimate for an observable $O$ is

$$
\langle O(\tau)\rangle_{\mathrm{QMC}}=\frac{\sum_{C_{n}}\left|w_{C_{n}}\right|\langle\langle O(\tau)\rangle\rangle_{C_{n}}}{\sum_{C_{n}}\left|w_{C_{n}}\right|}
$$

The expectation value in a single configuration reads

$$
\langle\langle O(\tau)\rangle\rangle_{C_{n}}=\frac{\prod_{\sigma}\left\langle T_{\tau}\left[\hat{n}_{i_{1} \sigma}\left(\tau_{1}\right)-\alpha_{\sigma}\left(b_{1}, s_{1}\right)\right] \ldots\left[\hat{n}_{i_{n} \sigma}\left(\tau_{n}\right)-\alpha_{\sigma}\left(b_{n}, s_{n}\right)\right] O_{\sigma}(\tau)\right\rangle_{0}}{\prod_{\sigma}\left\langle T_{\tau}\left[\hat{n}_{i_{1} \sigma}\left(\tau_{1}\right)-\alpha_{\sigma}\left(b_{1}, s_{1}\right)\right] \ldots\left[\hat{n}_{i_{n} \sigma}\left(\tau_{n}\right)-\alpha_{\sigma}\left(b_{n}, s_{n}\right)\right]\right\rangle_{0}} .
$$

The term in the denominator is proportional to $\left|w\left(C_{n}\right)\right|$ and is introduced to obtain the desired notation in eq. (5.23). For the single-particle Green function one obtains

$$
\left\langle\left\langle T_{\tau} c_{i}^{\dagger}\left(\tau_{1}\right) c_{j}\left(\tau_{2}\right)\right\rangle\right\rangle_{C_{n}}=G_{i j}^{0}\left(\tau_{1}, \tau_{2}\right)-\sum_{p, q=1}^{n} G_{i, i_{p}}^{0}\left(\tau_{1}, \tau_{p}\right)\left(M_{\sigma}^{-1}\right)_{p q} G_{i, i_{i_{q}}}^{0}\left(\tau_{q}, \tau_{2}\right) .
$$

This formula follows from eq. (5.24) and the fast matrix update formulas in appendix $B$. Equation 5.25 is the building block for all quantities that one wishes to calculate, because Wick's theorem holds in all configurations and hence all expectation values of creation and annihilation operators can be decomposed and rewritten in terms of the single-particle Green function.

\subsubsection{Two-Particle Measurements}

In the dual fermion method, two-particle quantities are particularly important. Here we show how they are measured.

The first thing to note is that for every configuration Wick's theorem can be 
applied. This leads to [105]

$$
\begin{aligned}
\left\langle c(\omega+\nu) c^{\dagger}(\omega) c\left(\omega^{\prime}\right) c^{\dagger}\left(\omega^{\prime}+\nu\right)\right\rangle_{0} & =g^{\mathrm{c}}(\omega+\nu, \omega) g^{\mathrm{c}}\left(\omega^{\prime}, \omega^{\prime}+\nu\right) \\
& -g^{\mathrm{c}}\left(\omega^{\prime}, \omega\right) g^{\mathrm{c}}\left(\omega+\nu, \omega^{\prime}+\nu\right) .
\end{aligned}
$$

Here, the dependence on spin indices has been ignored. Note that within a single Monte Carlo configuration, $g^{\mathrm{c}}$ is the value of the impurity Green function and it depends on two frequencies. Only after the QMC calculation becomes the impurity single-particle Green function $g$ diagonal in frequency space, i. e.

$$
g\left(\omega, \omega^{\prime}\right)=\left\langle g^{\mathrm{c}}\left(\omega, \omega^{\prime}\right)\right\rangle_{\mathrm{QMC}}=g(\omega) \delta_{\omega \omega^{\prime}}
$$

This is a consequence of time-translational invariance.

First, we look at the particle-hole Green function. $\chi_{\uparrow \uparrow ; \uparrow \uparrow}^{p h}$ and $\chi_{\uparrow \uparrow ; \downarrow \downarrow \downarrow}^{p h}$ are needed for the dual potential. Spin symmetry is used to improve the statistics of the measurement. For the first case the result is

$$
\begin{aligned}
\chi_{\uparrow \uparrow ; \uparrow \uparrow}^{p h}(\nu)_{\omega, \omega^{\prime}}= & -\frac{1}{2 \beta}\left[\left\langle g_{\uparrow}^{\mathrm{c}}\left(\omega^{\prime}, \omega\right) g_{\uparrow}^{\mathrm{c}}\left(\omega+\nu, \omega^{\prime}+\nu\right)+g_{\downarrow}^{\mathrm{c}}\left(\omega^{\prime}, \omega\right) g_{\downarrow}^{\mathrm{c}}\left(\omega+\nu, \omega^{\prime}+\nu\right)\right\rangle_{\mathrm{QMC}}\right. \\
& -\left\langle g_{\uparrow}^{\mathrm{c}}(\omega+\nu, \omega) g_{\uparrow}^{\mathrm{c}}\left(\omega^{\prime}, \omega^{\prime}+\nu\right)+g_{\downarrow}^{\mathrm{c}}(\omega+\nu, \omega) g_{\downarrow}^{\mathrm{c}}\left(\omega^{\prime}, \omega^{\prime}+\nu\right)\right\rangle_{\mathrm{QMC}} \\
& +\frac{1}{2}\left\langle g_{\uparrow}^{\mathrm{c}}(\omega+\nu, \omega)+g_{\downarrow}^{\mathrm{c}}(\omega+\nu, \omega)\right\rangle_{\mathrm{QMC}} \times \\
& \left.\times\left\langle g_{\uparrow}^{\mathrm{c}}\left(\omega^{\prime}, \omega^{\prime}+\nu\right)+g_{\downarrow}^{\mathrm{c}}\left(\omega^{\prime}, \omega^{\prime}+\nu\right)\right\rangle_{\mathrm{QMC}}\right] .
\end{aligned}
$$

Note that the disconnected part has to be subtract in the vertical channel. This follows from the definition of the particle-hole Green function and in section 4.1 we established that the vertex obtained from the disorder averaged particle-hole Green function is part of the dual fermion potential. For the second case the result is

$$
\begin{aligned}
\chi_{\uparrow \uparrow ; \downarrow \downarrow}^{p h}(\nu)_{\omega, \omega^{\prime}}= & \frac{1}{2 \beta}\left[\left\langle g_{\uparrow}^{\mathrm{c}}(\omega+\nu, \omega) g_{\downarrow}^{\mathrm{c}}\left(\omega^{\prime}, \omega^{\prime}+\nu\right)+g_{\downarrow}^{\mathrm{c}}(\omega+\nu, \omega) g_{\uparrow}^{\mathrm{c}}\left(\omega^{\prime}, \omega^{\prime}+\nu\right)\right\rangle_{\mathrm{QMC}}\right. \\
& -\frac{1}{2}\left\langle g_{\uparrow}^{\mathrm{c}}(\omega+\nu, \omega)+g_{\downarrow}^{\mathrm{c}}(\omega+\nu, \omega)\right\rangle_{\mathrm{QMC}} \times \\
& \left.\times\left\langle g_{\uparrow}^{\mathrm{c}}\left(\omega^{\prime}, \omega^{\prime}+\nu\right)+g_{\downarrow}^{\mathrm{c}}\left(\omega^{\prime}, \omega^{\prime}+\nu\right)\right\rangle_{\mathrm{QMC}}\right] .
\end{aligned}
$$

For the particle-particle channel the interaction of an up spin with a down spin is 
the contribution we need. This is the pairing channel. We have to calculate

$$
\begin{aligned}
\chi_{\uparrow \downarrow ; \uparrow \downarrow}^{p p}(\nu)_{\omega, \omega^{\prime}} & =\frac{1}{2 \beta}\left[\left\langle g_{\downarrow}^{\mathrm{c}}\left(-\omega,-\omega^{\prime}\right) g_{\uparrow}^{\mathrm{c}}\left(\omega+\nu, \omega^{\prime}+\nu\right)\right\rangle_{\mathrm{QMC}}\right. \\
& \left.+\left\langle g_{\uparrow}^{\mathrm{c}}\left(-\omega,-\omega^{\prime}\right) g_{\downarrow}^{\mathrm{c}}\left(\omega+\nu, \omega^{\prime}+\nu\right)\right\rangle_{\mathrm{QMC}}\right] .
\end{aligned}
$$

To shorten the notation for the particle-particle channel, we introduce

$$
\chi^{p}(\nu)_{\omega, \omega^{\prime}+\nu} \equiv \chi_{\uparrow \downarrow \uparrow \uparrow \downarrow}^{p p}(\nu)_{\omega, \omega^{\prime}}
$$

We can now exploit crossing symmetry to obtain

$$
\chi_{\uparrow \downarrow ; \downarrow \uparrow}^{p p}(\nu)_{\omega, \omega^{\prime}}=-\chi^{p}(\nu)_{\omega,-\omega^{\prime}-\nu} .
$$

Using eq. (D.15) and SU(2) symmetry, it is now possible to obtain the two-particle Green function and vertex function for all possible spin configurations that we may need.

\subsubsection{Disorder-Averaged Quantities from Monte Carlo Simulations}

In the above, we looked at the measurement of one- and two-particle Green functions for one disorder configuration. The solution of the disordered impurity problem requires that we perform a disorder-average. The disorder-average is performed after the Monte Carlo simulation is converged for a given configuration and the disorder-averaged expectation value is

$$
\{O\}=\left\{\langle O\rangle_{\mathrm{QMC}}\right\}=\frac{1}{N_{\mathrm{Dis}}} \sum_{i=1}^{N_{\mathrm{Dis}}}\langle O\rangle_{\mathrm{QMC}}^{i}
$$

for an observable $O$, where $\{\ldots\}$ denotes the disorder average over configurations $i$, $N_{\text {Dis }}$ is the number of disorder configurations used and $\langle\ldots\rangle_{\mathrm{QMC}}^{i}$ denotes the Monte Carlo estimate for a disorder configuration $i$. This is illustrated in figs. 5.1 and 5.2 .

For the dual potential we need the disorder two-particle Green function. It is obtained according to

$$
\{g(\omega+\nu) g(\omega)\}=\frac{1}{N_{\text {Dis }}} \sum_{i=1}^{N_{\text {Dis }}} g^{i}(\omega+\nu) g^{i}(\omega)
$$

for the particle-hole channel. $g^{i}$ denotes the impurity Green function obtained by the solver for the disorder configuration $i$. This quantity depends on only two frequencies and does not require additional measurements in the Monte Carlo run, 
therefore it is much cheaper than the interacting two-particle Green functions. The measurement is illustrated in fig. 5.3 .

\subsection{Summary}

In this chapter we have motivated the use of continuous-time quantum Monte Carlo and introduced the basic ideas of Monte Carlo methods. The interaction expansion algorithm (CT-INT) was discussed in more detail as this is the algorithm that was used in this thesis. CT-INT relies on an expansion in terms of the interaction part of the Hamiltonian and is in principle exact. However, it is best for small to intermediate interaction strength as the size of the configuration matrix growth as $U^{3}$ 44.

In section 5.2 .3 the measurement of two-particle quantities was explained as these quantities, namely the two-particle Green functions, are key to the dual fermion approach. In the end, we combined the interacting impurity problem with the disorder-average that is needed for DMFT, DCA and dual fermions. The disorderaverage is performed over multiple realization of the disorder for the impurity or cluster problem.

This concludes the discussion of numerical methods and models. The following two chapters present results for the Anderson-Falicov-Kimball model and AndersonHubbard model that were obtained by means of the dual fermion approach for disordered interacting systems. 


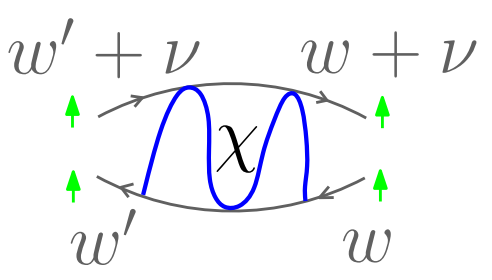

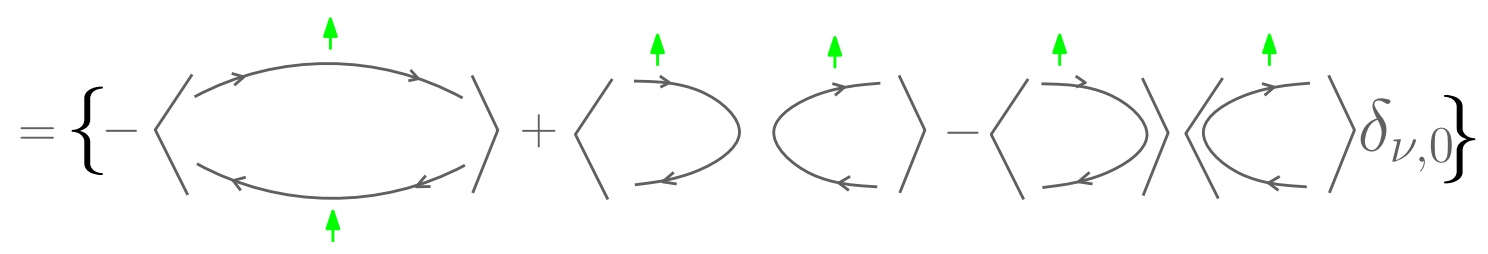

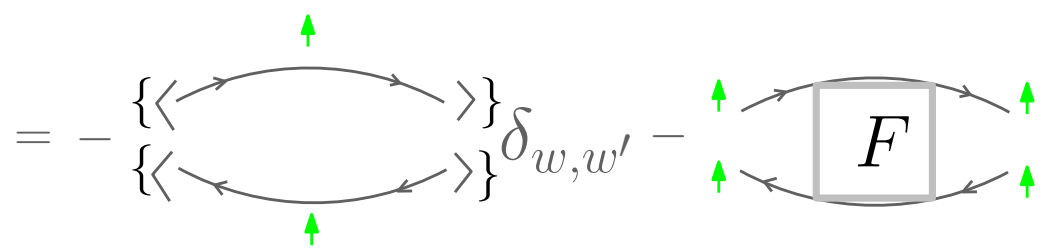

$$
w^{\prime+\nu} w_{w}^{\prime+v}
$$

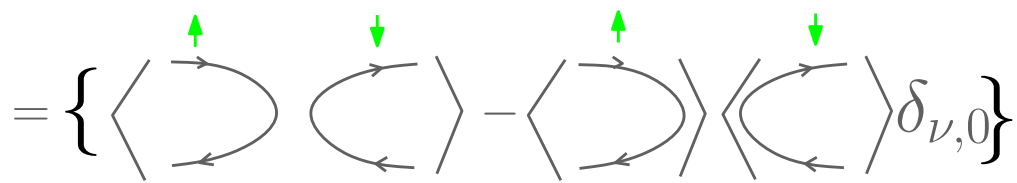

Figure 5.1: Definition of the particle-hole Green functions in terms of the Monte-Carlo measurement. $\langle\ldots\rangle$ denotes a Monte Carlo average for a single configuration, $\{\ldots\}$ denotes the disorder average (Based on illustration by S.-X. Yang [106]). 


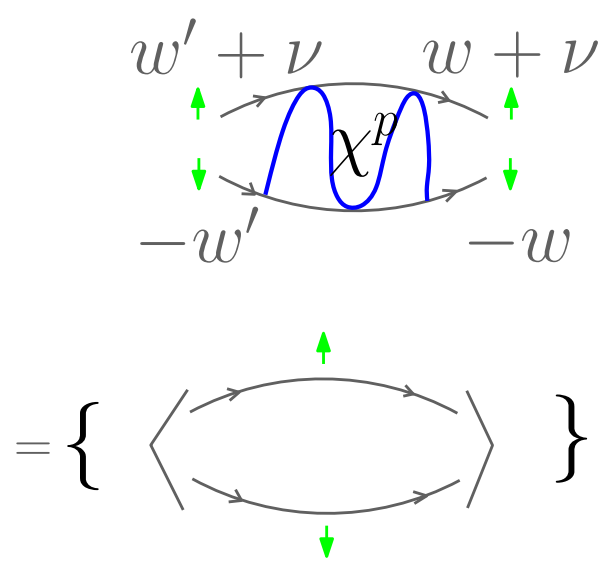

Figure 5.2: Definition of the two-particle pairing Green function in terms of the MonteCarlo measurement. $\langle\ldots\rangle$ denotes a Monte Carlo average for a single configuration, $\{\ldots\}$ denotes the disorder average (Based on illustration by S.-X. Yang [106]).

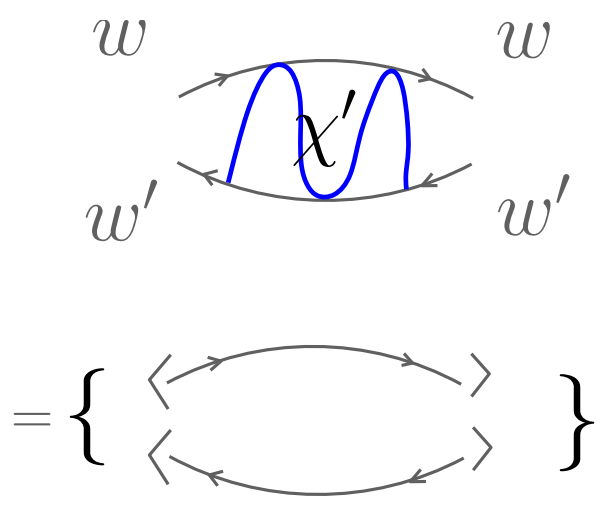

Figure 5.3: Definition of the particle-hole disorder Green function in terms of the Monte Carlo measurement. For this illustration spins have been omitted as the result does not depend on the spin configuration (Illustration by S.-X. Yang [106]). 



\section{Test Case for the Dual Fermion Algorithm: The Anderson-Falicov-Kimball Model}

Material published in S.-X. Yang, P. Haase, H. Terletska, Z. Y. Meng, T. Pruschke, J. Moreno, and M. Jarrell. Dual-fermion approach to interacting disordered fermion systems. Phys. Rev. B, 89:195116, May 2014

A first test for our algorithm is the Anderson-Falicov-Kimball model, which was introduced in section 2.2.1 and has the Hamiltonian

$$
H_{F K}=\sum_{k}\left(\epsilon_{k}-\mu\right) c_{k}^{\dagger} c_{k}+U \sum_{i} n_{i}^{c} n_{i}^{f}-\sum_{i} v_{i} n_{i}^{c}
$$

It is a good test case, because the solution of the impurity problem is trivial and thus the computational cost is low. The particle-particle interaction is static and leads to a vertex function that depends only on two frequencies. Dynamical mean-field theory (DMFT) gives a temperature-independent solution. This is because DMFT for the Anderson-Falicov-Kimball model at half-filling is equivalent to DMFT for the Anderson model, which has no dynamics and is thus temperature-independent. This is not in accordance with results from the dynamical cluster approximation (DCA). Thus, it is clear that dual fermions have to recover a temperature-dependent solution to be considered an improvement over DMFT.

First, the 1d system is addressed. Comparisons with DCA calculations are easily done because DCA is relatively cheap in 1d. We examine the local Green function to observe how well the dual fermion approach performs compared to DCA and to get a first idea of the influence of disorder. Next, we present results for the relative corrections compared to DMFT results for the local Green function. The corrections allow us to identify in which parameter region non-local correlations are important.

Second, we move on to the $2 \mathrm{~d}$ system and calculate a phase diagram on the $U V$ plane. To this end, we calculate the difference

$$
\operatorname{Im} \delta G=\operatorname{Im} G_{l o c}(3 i \pi T)-\operatorname{Im} G_{l o c}(i \pi T) .
$$

The sign of $\operatorname{Im} \delta G$ indicates a qualitative change in the local Green function from metallic behavior to more insulator-like behavior. This quantity is not suited to 

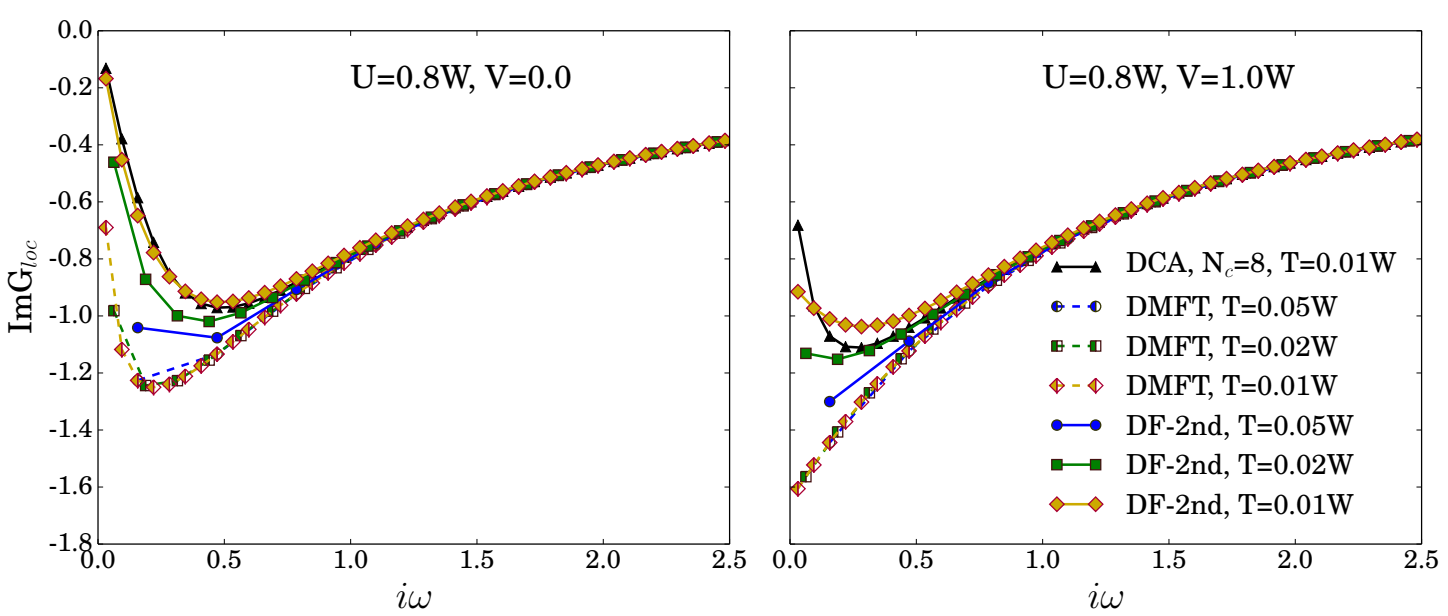

Figure 6.1: The local Green function obtained from DMFT and dual fermions for $U=$ $0.8 \mathrm{~W}$ and various temperatures. Results for the clean system are shown on the left, and results for disorder with $V=1.0 \mathrm{~W}$ on the right. For the lowest temperature $T=0.01 \mathrm{~W}$ we added DCA results for comparison. (Data from [106])

study the Anderson transition as it does not distinguish between localized and extended states. Box disorder cannot open a gap in the spectrum and thus $\operatorname{Im} \delta G$ cannot become negative. We supplement the Green function data by the conductivity and find that it is strongly reduced for large values of the disorder strength $V$.

In the following, all results were obtained for half-filling of the system with $c$ and $f$ electrons. The disorder is distributed according to a box probability distribution $p_{\text {Box }}\left(v_{i}\right)=\frac{1}{V} \Theta\left(\frac{V}{2}-\left|v_{i}\right|\right)$, where $V$ denotes the strength of the disorder. In plots and equations, DF stands for the dual fermion approach, DF-2nd represents the second order approximation and DF-FLEX the dual fermion FLEX, which is sometimes reduced to FLEX in the text. The dispersion relation reads $\epsilon_{k}=-2 t \sum_{i=1}^{d} \cos \left(\frac{2 \pi}{L_{i}} k_{i}\right)$, where $t=0.25$ is the hopping constant, $d$ the dimensionality, $L_{i}$ the linear dimension of the lattice in direction $i$ and $k_{i}$ the $i$ th component of the momentum vector.

In [135] the dual fermion approach has been applied to the clean Falicov-Kimball model.

\subsection{Dual Fermion Corrections in 1d}

We start by examining the imaginary part of the local Matsubara Green function for a $1 \mathrm{~d}$ system shown in fig. 6.1. The bandwidth is $W=1 \mathrm{in} 1 \mathrm{~d}$. For the clean system $(V=0)$ in fig. 6.1, the solutions display insulator-like behavior. This can 

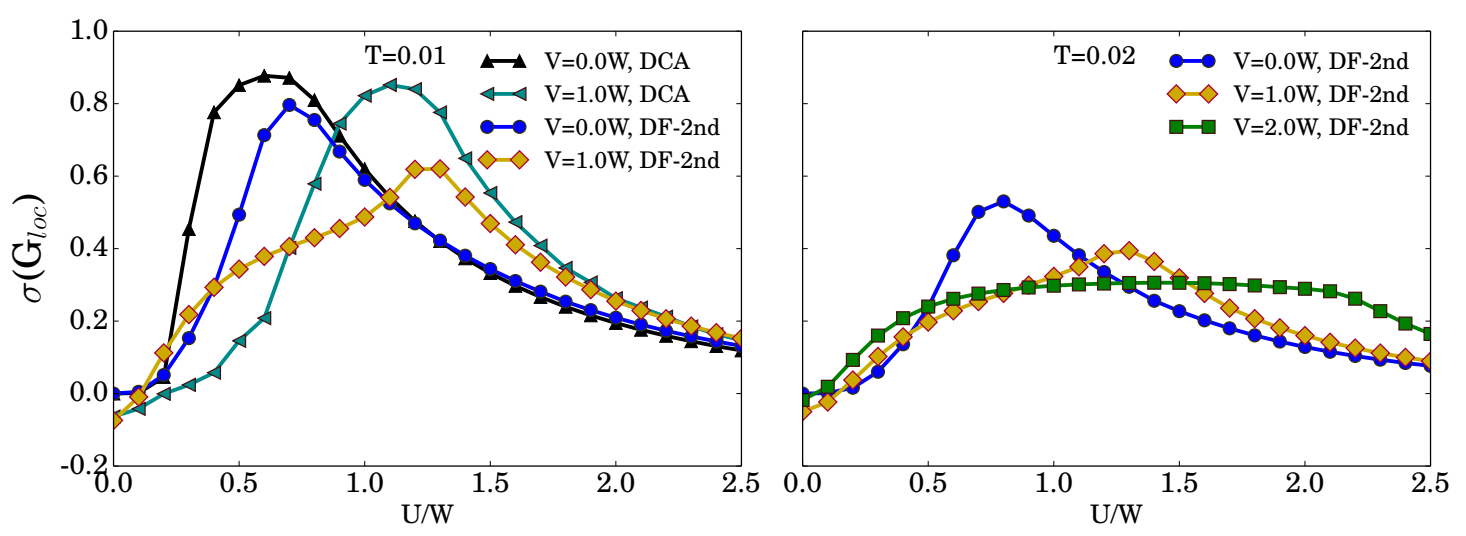

Figure 6.2: Relative corrections to the DMFT result for the local Green function at $\omega=i \pi T$. The results on the left are for $T=0.01 \mathrm{~W}$ and are compared to DCA results. On the right, the results are for $T=0.02 \mathrm{~W}$ and show how the corrections from dual fermions change with increasing disorder strength. (Dual fermion data from [106.)

be inferred from $\operatorname{Im} G_{\text {loc }}(i \pi T)$ going to zero for decreasing temperature. The DMFT solutions are essentially on one curve, indicating that the result is temperatureindependent. This is in contrast with the dual fermion results which show a clear temperature-dependence.

To obtain an estimate for the quality of the results we add a DCA solution for $N_{c}=8$ for the lowest temperature $T=0.01 W$. The dual fermion and DCA results are essentially the same. These results show that the temperature dependence in the Falicov-Kimball model at half-filling is strongly linked with non-local correlations [106].

For the interacting system with disorder, $\operatorname{Im} G_{\text {loc }}$ is shown in the right panel of fig. 6.1. The DMFT result is again essentially temperature-independent and shows metal-like behavior. For the dual fermions, the low-temperature result shows insulating behavior and for increasing temperatures we observe a transition to a metallic solution.

The DCA solution for the disordered interacting system shows the same trend as the dual fermion result, namely an increase in the absolute value of $\operatorname{Im} G_{\text {loc }}(i \pi T)$ compared to the clean system, but the agreement with the dual fermion result is not as good as for the clean system. However, the results underline the importance of the non-local correlations for the physics of the Anderson-Falicov-Kimball model, which appear even stronger in case of a disordered interacting system.

To get a better understanding of how and where non-local correlations change the 
DMFT result we look at the relative correction

$$
\sigma\left(G_{\mathrm{loc}}\right)=\frac{\operatorname{Im} G_{\mathrm{loc}}^{\mathrm{NL}}(i \pi T)-\operatorname{Im} G_{\mathrm{loc}}^{\mathrm{DMFT}}(i \pi T)}{\left|\operatorname{Im} G_{\mathrm{loc}}^{\mathrm{DMFT}}(i \pi T)\right|},
$$

where NL stands for either dual fermions or DCA.

We observe a general agreement with the DCA result for $T=0.01 \mathrm{~W}$ for the clean system in fig. 6.2 on the left panel. The main difference is that the corrections from DCA are larger for small values of $U$. In the large $U$ limit DCA and dual fermions are nearly on top of each other. For the disordered interacting system, the dual fermions overestimate the correction compared to the DCA calculation for small values of $U$. In fig. 6.2 on the right panel the temperature has been increased to $T=0.02 \mathrm{~W}$ with the effect that the relative corrections are decreased. From both plots in fig. 6.2 we can see that the corrections are strongest for intermediate values of $U$. For large values of $U$ the model approaches the atomic limit and DMFT becomes a good approximation which is apparent from the decreasing of $\sigma\left(G_{\text {loc }}\right)$.

Increasing disorder moves the maximum correction to larger values of $U$, which is seen from both the dual fermion and DCA results. At least in the case of the dual fermion results, disorder broadens the region for which the corrections are significant.

The main message of this section is that dual fermions capture important effects from non-local correlations and significantly improve the DMFT results [106]. Dual fermion results agree with DCA results qualitatively for the disordered interacting system. For the clean system, there often is a remarkable quantitative agreement as well.

\subsection{2d Phase Diagram}

In this section our aim is to extract a phase diagram on the $U V$-plane for the $2 \mathrm{~d}$ system for which the bandwidth is $W=2$. We examine $\operatorname{Im} \delta G$ (eq. (6.2) ) as an easily accessible quantity, which allows us to assess whether the Green function is metallic or insulator-like. Insulator-like means $\operatorname{Im} \delta G$ is negative, i. e. the imaginary part of the local Green function has a minimum and $\operatorname{Im} G_{l o c}(i \pi T)$ converges to zero as $T \rightarrow 0$. Results are shown in fig. 6.3.

Strictly speaking, this criterion only holds at zero temperature. We thus refrain from calling the phase an insulator but rather call it a bad metal [106]. For the clean system this agrees with reference [135]. We find that for box disorder we can detect a transition for large values of $U$, but we find no sign of Anderson-localization for large values of $V$. The arithmetic averaging used in the dual fermion formalism does not distinguish between localized and extended states and thus cannot capture the transition on the single-particle level.

To understand the driving force behind the localization for a small disorder parameter, we plot in fig. 6.4 the leading eigenvalue (LEV) $\lambda$ of the equation $\Gamma \chi_{0} \phi=\lambda \phi$ 


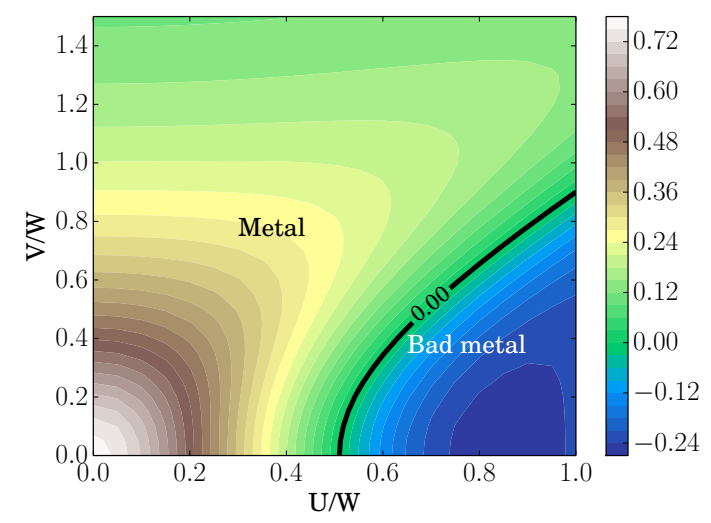

(a) DMFT

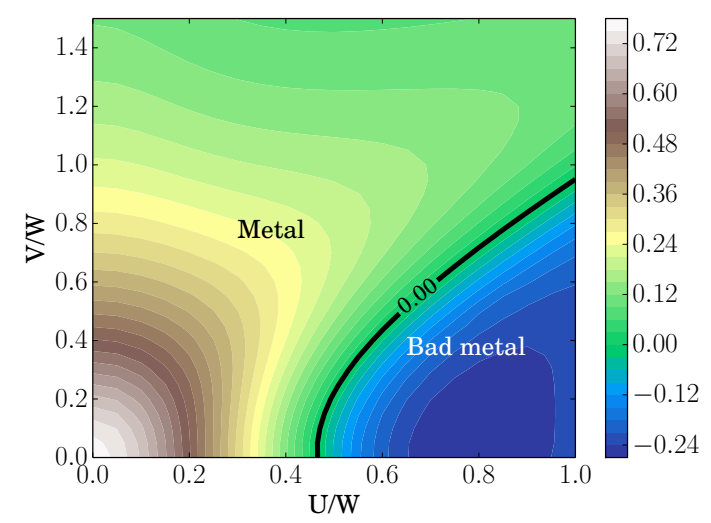

(b) DF-2nd

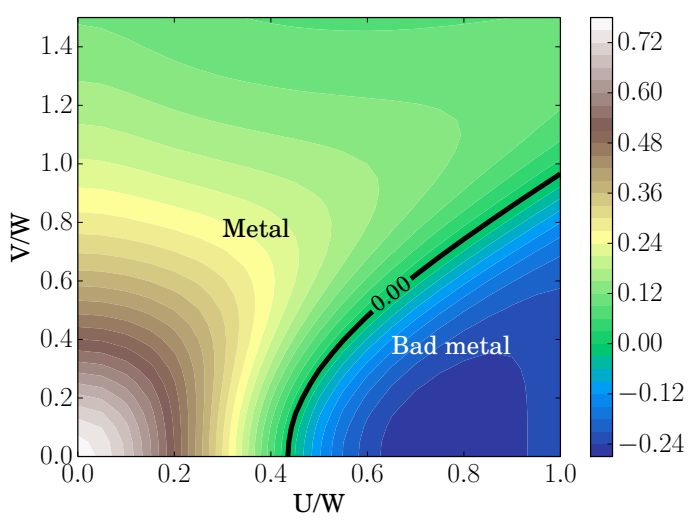

(c) DF-FLEX

Figure 6.3: Phase diagram on the $U V$-plane from $\operatorname{Im} \delta G$ from (a) DMFT, (b) 2nd order dual fermions and (c) dual fermion FLEX for $T=0.025 \mathrm{~W}$. (Data from [106]) 


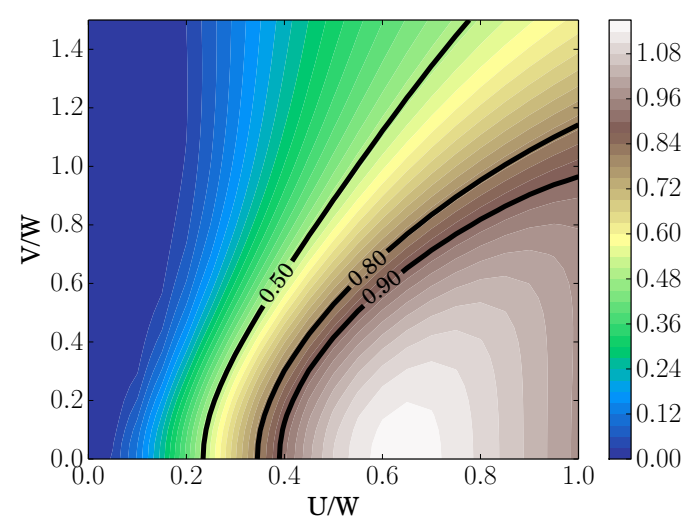

(a) DMFT

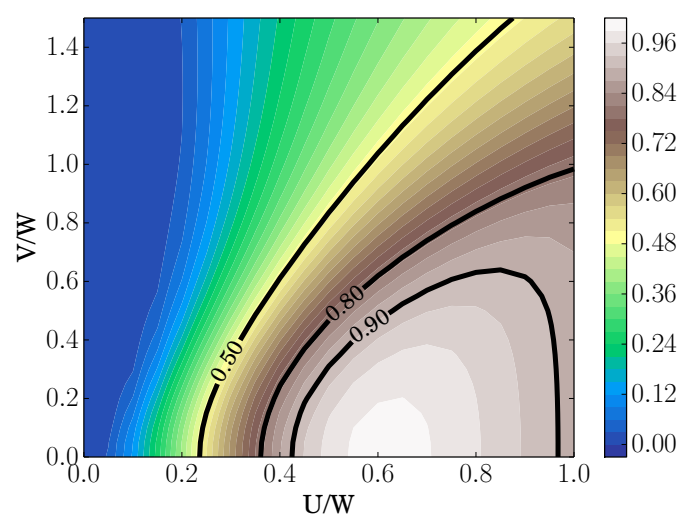

(b) DF-2nd

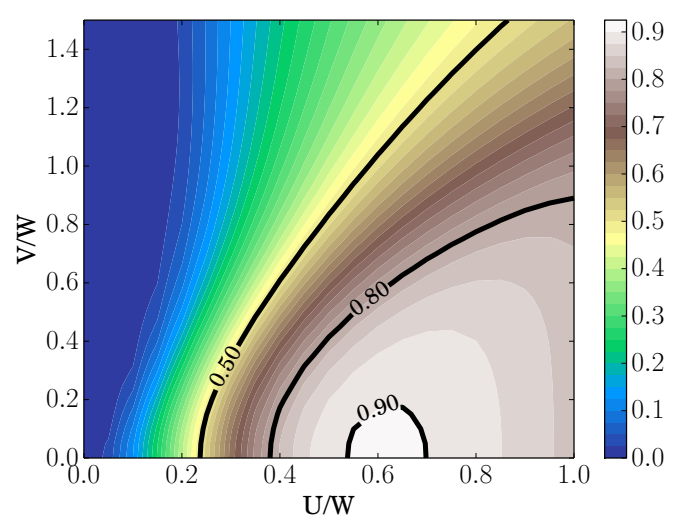

(c) DF-FLEX

Figure 6.4: Leading eigenvalue of the charge-density channel on the $U V$-plane from (a) DMFT, (b) 2nd order dual fermions and (c) dual fermion FLEX for $T=$ $0.025 W$. (data from [106]) 
for the charge density channel, where $\Gamma$ is the irreducible vertex and $\chi_{0}$ the bare lattice susceptibility. We find that the LEV is close to one around the metal-badmetal crossover. This shows that the bad metal in the vicinity of the clean system is susceptible to the formation of a charge-density-wave.

$\operatorname{Im} \delta G$ is only a rough estimate, therefore we supplement it by calculating the dc conductivity as well. We include vertex corrections, thereby taking into account corrections to the bubble diagram, which contains the lowest order contributions. The conductivity is shown in fig. 6.5 which reveals that the conductivity decreases for large values of $U$ and $V$, but always remains finite. This can be due to the approximation, as well as the fact that at the temperature under consideration the system is indeed merely a bad metal and not yet an insulator. We fix the critical value of the conductivity by determining $U_{c}$ for $V=0$. The corresponding value of the conductivity is $\sigma_{d c}=0.04$. We find a smooth connection on the $U V$-plane from the $U$-axis to the $V$-axis, thereby determining an estimate for the phase boundary in the whole plane. For $V \ll \frac{W}{2}$ the phase diagram from $\sigma_{d c}$ and $\operatorname{Im} \delta G$ are very similar and show that small amounts of disorder help to mobilize the charge carriers. For strong disorder and weak interaction the slopes of the lines of equal conductivity are negative, indicating that interactions help to localize the $c$-electrons [106]. This behavior is in contrast with the result from [41, 136]. Those results were obtained within DMFT on the infinite dimensional Bethe lattice for the ground-state phase diagram, whereas we work in $2 \mathrm{~d}$ and for $T>0$. More importantly, however, we take into account non-local correlations through our dual fermion formalism. As we have seen already in the previous sections, especially the latter can become quite important for stronger disorder, thus explaining the difference. Let us stress that it is possible that we nevertheless still miss the correct behavior as Anderson localization is not a self-averaging effect.

\subsection{Summary}

The Anderson-Falicov-Kimball model served as a first test for the new formalism. We started by looking at the local single-particle Green function and how it compares to DMFT and DCA. We found that dual fermions significantly improve the DMFT results and compare quite well with the DCA results. The best agreement is achieved for the clean or weakly disordered system. For strong disorder dual fermions and DCA still show qualitative agreement but quantitatively we observe noticeable deviations, not just in value but also in the qualitative line shape of the corrections.

For the Anderson-Falicov-Kimball model in 2d the dual fermion approach gives good results for weak disorder. However, in the region of strong disorder it possibly fails to produce the correct phase diagram. This is not completely unexpected as the arithmetic averaging is not able to detect Anderson localization. Also, for strong 


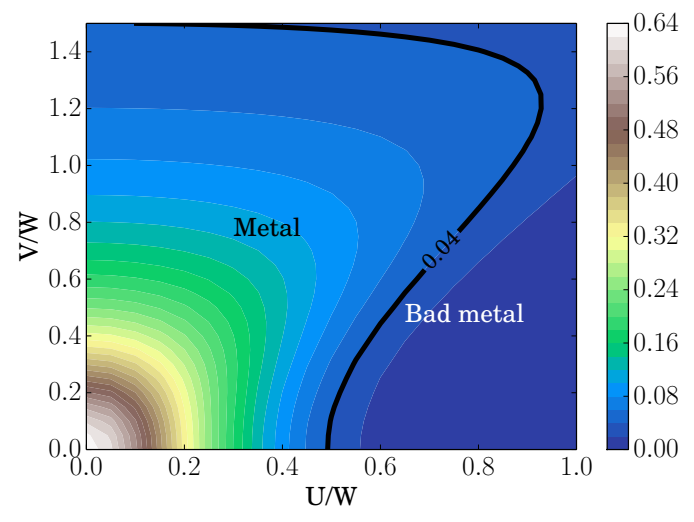

(a) DMFT

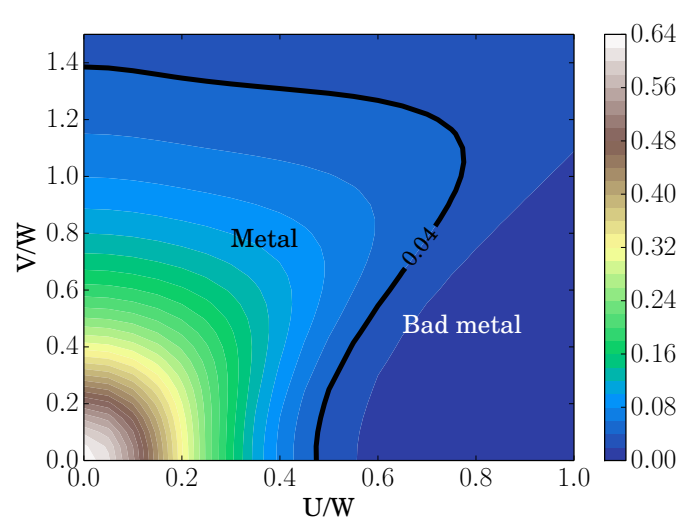

(b) DF-2nd

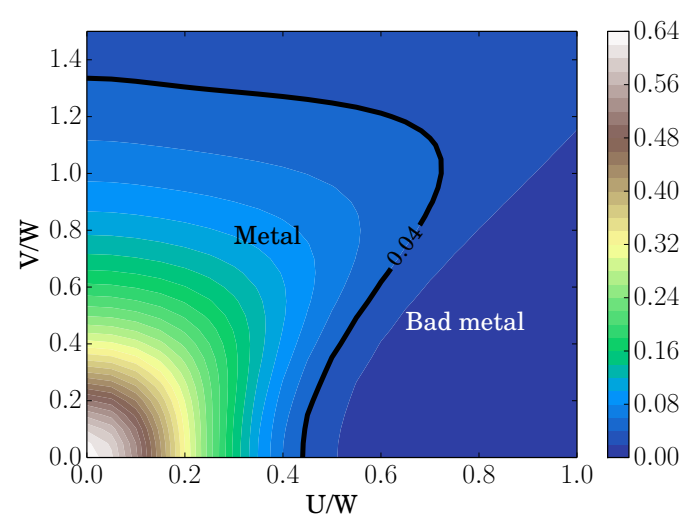

(c) DF-FLEX

Figure 6.5: Phase diagram from the conductivity from (a) DMFT, (b) 2nd order dual fermions and (c) dual fermion FLEX for $T=0.025 \mathrm{~W}$. (data from [106]) 
disorder the quantitative agreement between DCA and dual fermions is reduced, which might indicate that dual fermions miss some important contributions.

For the Anderson-Hubbard model in the next section, the shape of the metallic region for strong disorder is qualitatively different and does indeed meet the expectations based on the previous work in reference [137]. We suspect that the particularly bad behavior of the DMFT, i. e. temperature independent Green functions, provides a bad starting point for the dual fermions in case of the Anderson-Falicov-Kimball model at half-filling. A bad starting point means that the small parameter for the perturbative expansion is actually not as small as one would like. As the solution of the impurity problem and the disorder-averaging look the same, it is possible that the disorder vertex and the interaction vertex act together in a way such that the effective expansion parameter is not small. However, we did not find a way to verify that this conjecture is true. 



\section{Results for the Anderson-Hubbard Model}

In this chapter, we present results for the Anderson-Hubbard model that were obtained using the dual fermion approach. The Anderson-Hubbard model was introduced in section 2.2.1 and has the Hamiltonian (c.f. eq. 2.5)

$$
H_{A H}=-\sum_{i j, \sigma}\left(t_{i j}+\mu \delta_{i j}\right)\left(c_{\sigma i}^{\dagger} c_{\sigma j}+h . c .\right)-\sum_{i, \sigma} v_{i} n_{i \sigma}+U \sum_{i}\left(n_{i \uparrow}-\frac{1}{2}\right)\left(n_{i \downarrow}-\frac{1}{2}\right) .
$$

In section 7.1 we compare the dual fermion results with dynamical cluster approximation (DCA) results in 1d to see how well dual fermions perform compared to established cluster methods. First, we look at the imaginary part of the local Green function $\mathrm{ImG}_{\text {loc }}$ to get a general idea of the quality of the dual fermion approach. Second, we look at the relative corrections to the dynamical mean-field theory (DMFT) result for $\operatorname{ImG}_{\text {loc }}(i \pi T)$. This quantity allows to show results for a wide range of parameters in compact form.

In section 7.2 we continue with results for the $3 \mathrm{~d}$ system. We opt for the $3 \mathrm{~d}$ system because it displays an Anderson transition, a finite temperature transition from the paramagnet to the antiferromagnet and a Mott metal-insulator transition for the paramagnetic Hubbard model. All three transitions are investigated within the dual fermion approach. We find quantitative corrections as well as qualitative ones compared to the DMFT results, e.g. for the temperature dependence of the Mott metal-insulator transition in section 7.2.2.

All results were obtained for the half-filled system. If not mentioned otherwise, we use binary disorder and second order dual fermions. The dispersion relation reads $\epsilon_{k}=-2 t \sum_{i=1}^{d} \cos \left(\frac{2 \pi}{L_{i}} k_{i}\right)$, where $t=0.25$ is the hopping constant, $d$ the dimensionality, $L_{i}$ the linear dimension of the lattice in direction $i$ and $k_{i}$ the $i$ th component of the momentum vector. In plots and equations, DF stands for the dual fermion approach, DF-2nd represents the second order approximation and DF-FLEX the dual fermion FLEX, which is sometimes reduced to FLEX in the text. 

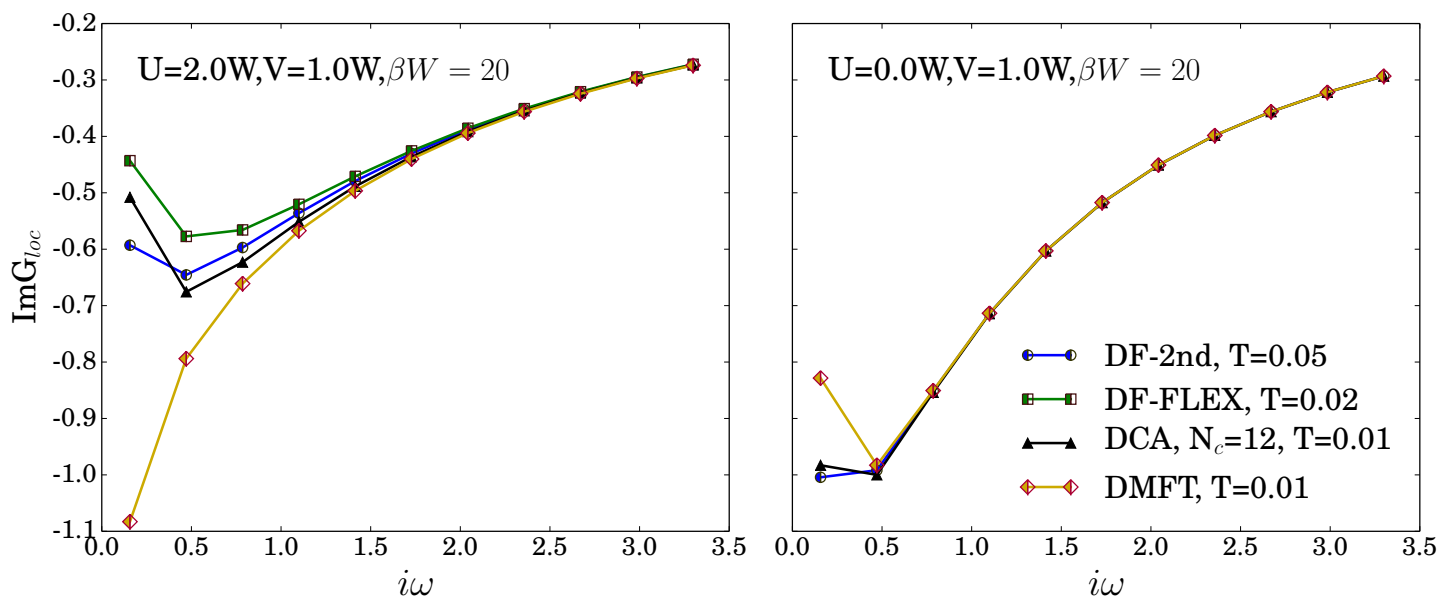

Figure 7.1: Imaginary part of the dual Green function for $\beta W=20$ and $V=1.0$. On the left we have $U=2 W$ and on the right $U=0$. For $U=0$ the dual fermion FLEX calculation did not converge.

\subsection{1d Results}

The one-dimensional case is the worst-case scenario for methods that retain a meanfield character as non-local correlations become particularly important in 1d. Nevertheless, it is a good test ground for the dual fermion approach, as it allows for a comparison with DCA. This is because DCA calculations in 1d are relatively cheap for reasonably large cluster sizes. The performance of DCA in 1d [138] is well known, so we can estimate the quality of the dual fermion results. But what we are really interested in is the comparison of the dual fermion approach with established cluster approximations, as these are the competitors of our approach.

The goal is to establish a reliable alternative technique for simulations off halffilling when quantum Monte Carlo based methods are needed, which is often the case in more than one dimension. The expectation is that if we are able to capture the DCA results with some accuracy in 1d, we should expect the same in higher dimensions. Also, the overall quality of the approximation should improve in higher dimensions as the importance of non-local correlations is reduced, and the results become more mean-field like.

First, we take a look at the imaginary part of the local Green function $\operatorname{ImG}_{\text {loc }}$. Figure 7.1 shows examples of $\mathrm{ImG}_{\text {loc }}$ from various approximations for $U=2.0 \mathrm{~W}$ and $V=1.0 \mathrm{~W}$. The bandwidth is $W=1.0$ in our units. Both dual fermion results and the DCA result show insulator-like behavior in the left panel of fig. 7.1, i.e. $\mathrm{ImG}_{\text {loc }}$ has a minimum. Dynamical mean-field theory (DMFT) gives a metallic solution. The plot on the right of fig. 7.1 shows results for the non-interacting system. DMFT overestimates the effect of the disorder, showing an insulator-like 


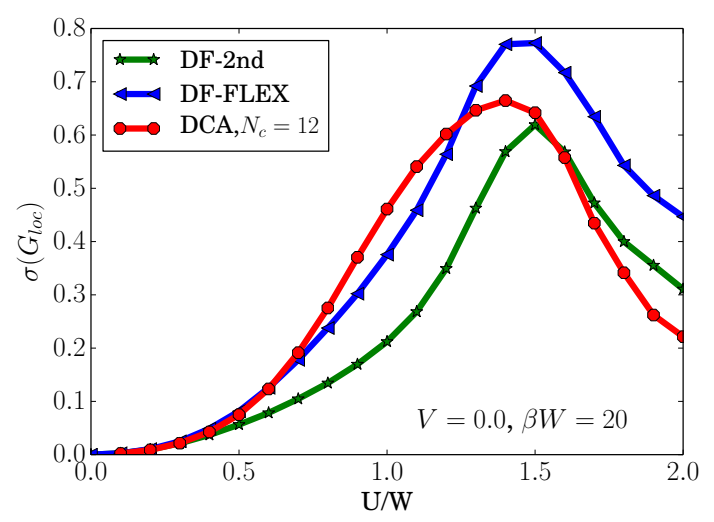

(a)

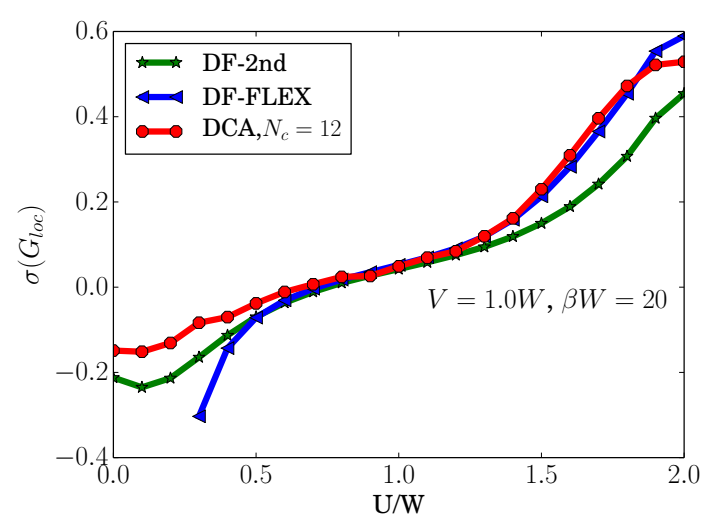

(c)

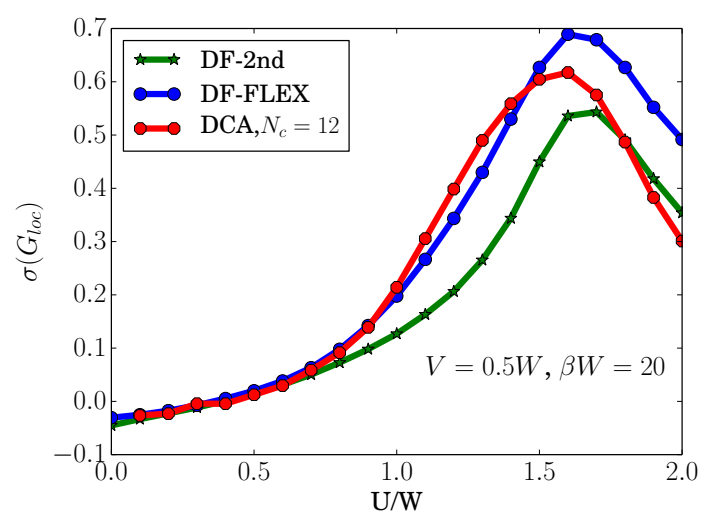

(b)

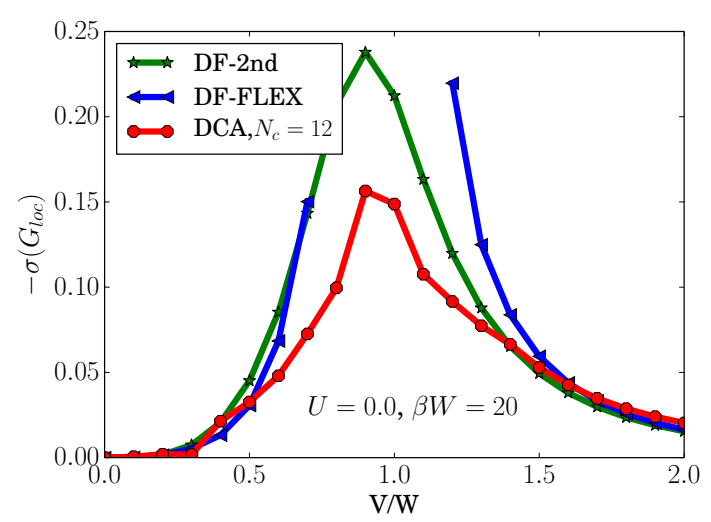

(d)

Figure 7.2: Relative correction as a function of $U$ for multiple values of $V$ for binary disorder at $\beta W=20$.

solution. Both second order dual fermions and DCA show a solution on the edge between metallic and insulator-like behavior. There is no FLEX result, as the FLEX calculation did not converge.

For a more systematic study of the effect of non-local correlations we compare dual fermions and DCA by looking at the relative corrections compared to the DMFT result according to eq. 6.3). The relative corrections are a measure for the importance of non-local correlations. Results are shown in figs. 7.2 and 7.3 .

In all plots results for 2nd order dual fermions, dual fermion FLEX and DCA with $N_{c}=12$ are shown. With increasing interaction strength (figs. 7.2a to 7.2c the correction increase until a maximum is reached. For the clean system (fig. 7.2a) and the weakly disordered system (fig. 7.2b) the strongest correction are around $1.5 \mathrm{~W}$. This is the region of the metal-insulator transition when non-local correlations are included, whereas the transition happens in DMFT for larger values of $U$. With 


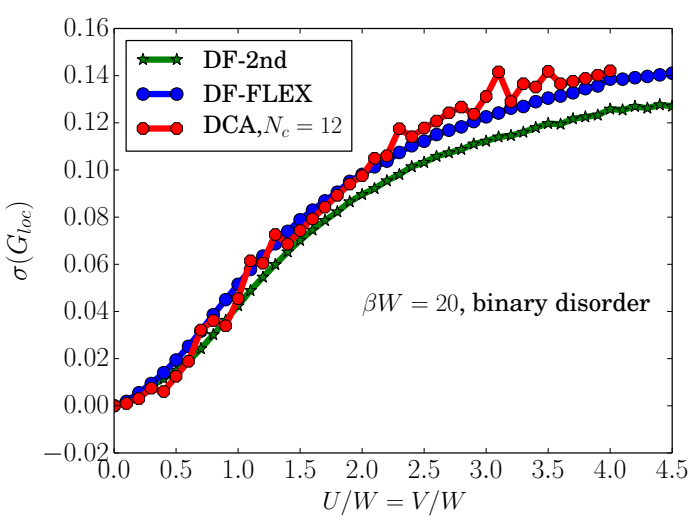

(a)

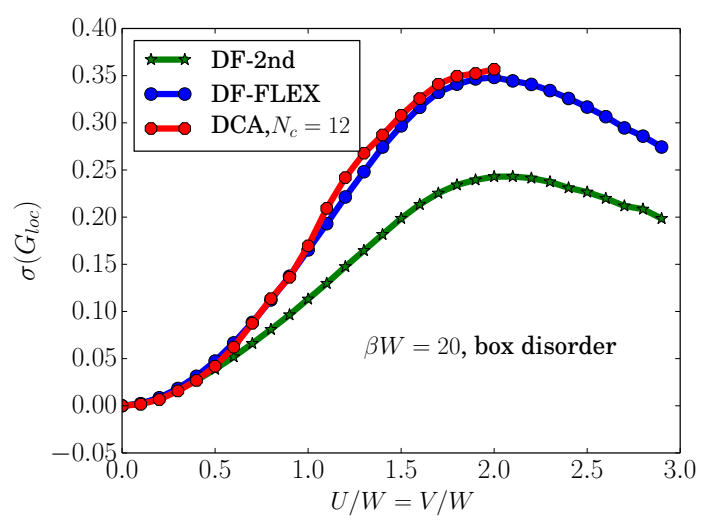

(b)

Figure 7.3: Relative correction of $\operatorname{Im} G_{l o c}(i \pi T)$ with respect to the DMFT as a function of $U=V$ for (a) binary and (b) box disorder for $\beta W=20$.

increasing disorder strength (from fig. $7.2 \mathrm{a}$ to fig. 7.2c the maximal corrections are reduced from about 80 percent to about 60 percent for the FLEX and shifted to larger values of $U$ from $1.4 W$ to $2 W$.

Figure $7.2 \mathrm{~d}$ shows corrections for the non-interacting system. The picture is the same as for the interacting system but this time the maximal corrections occur slightly below the bandwidth $W$. In figs. $7.2 \mathrm{c}$ and $7.2 \mathrm{~d}$ the FLEX calculations did not converge around $V=W$ and weak interactions. This is the region where binary disorder opens a gap. We did not observe this problem for the Mott gap and a possible explanation is that disorder opens the gap quicker than the Hubbard interaction does, leading to stronger fluctuations that interfere with the FLEX calculation.

In fig. 7.3 results are shown for the special case $U=V$. For binary disorder the maximal corrections we found were small, i. e. below 15 percent. At $U=V=$ $4.5 \mathrm{~W}$ the maximum has not yet been reached. For box disorder the corrections are significantly larger, the maximal corrections are reached around $U=V \lesssim 2 W$ and are about 35 percent.

The qualitative agreement between all three approaches is good, except for the non-convergent FLEX results. In fig. 7.2 the FLEX results show good quantitative agreement with the DCA results below the interaction strength $U_{M}$ of the maximal corrections. Above $U_{M}$ the 2 nd order results show better agreement, but are not as good as the FLEX for $U<U_{M}$. Exceptionally good agreement is found between FLEX and DCA for $U=V$ in fig. 7.3. The results are nearly on top of each other, whereas the 2 nd order results show qualitative agreement but otherwise underestimate the corrections, especially pronounced for box disorder.

We use the results in fig. 7.2 to compare the numerical efficiency. For the case of binary disorder for $U=4 W$ the computational effort to obtain the DCA result is 
roughly ten times higher than for the dual fermion FLEX. The reason is that for binary disorder there are only two disorder configurations for dual fermions but 4096 configurations for DCA with $N_{c}=12$. We sampled 200 random configurations for DCA which led to a converged solution with respect to the number of configurations. Still, the DCA data is much noisier than the dual fermion data. In case of box disorder, the computational effort becomes comparable. The dual fermion calculation requires a larger number of configurations whereas DCA needs less configurations compared to binary disorder as the different random configurations are more similar to each other. In that case the efficiency depends on the specific parameters and the number of iterations needed. It can be expected that dual fermions will gain an edge over DCA as the temperature is lowered and the sign problem becomes more severe. We had to abort the DCA calculations at smaller values of $U=V$ as we were not able to obtain converged results. This is probably caused by the sign problem.

In figs. $7.2 \mathrm{c}$ and $7.2 \mathrm{~d}$ the weakness of the dual fermion FLEX approximation becomes apparent. In the case of binary disorder around $V=W$ the FLEX calculation does not converge. This is around the transition from a metallic local Green function to an insulating one. The failure is not apparent from the leading eigenvalue of the Bethe-Salpeter equation. This underlines the fact that the FLEX approximation has to be used with care. As we can see from the small $U$ limit in fig. $7.2 \mathrm{c}$ the FLEX result becomes much worse than the 2 nd order approximation but still converges. It is therefore advisable to always check the FLEX results against the 2nd order results for consistency and look at surrounding data points to see whether any divergences are close by.

The physical interpretation of the results is that disorder reduces the effect of nonlocal correlations from the Hubbard term [24]. This is apparent from the reduced maximal correction. Also, stronger interactions are necessary for maximal non-local correlations. This result confirms our expectations. If the underlying lattice does not show long-range order, it is natural to expect that the electrons on the lattice do not show long-range order either.

We conclude that the dual fermion approach is able to recover non-local correlations on a level that is comparable to DCA. Thus, we can expect good qualitative and quantitative results in higher dimensions. If applicable, the FLEX approximation is the method of choice for small to intermediate values of $U$ and weak disorder. For larger values of $U$ the 2 nd order approximation gives results closer to DCA. For a final evaluation comparisons with other quantum cluster methods are desirable, but go beyond the scope of this thesis.

For the case of binary disorder the FLEX has to be handled with care as it can show non-convergent behavior and give nonsensical results. We point out that the dual fermion approach gives better results in the presence of disorder for the Anderson-Hubbard model than for the Anderson-Falicov-Kimball model. The reason is possibly that for the Hubbard model the DMFT solution is already a better 
approximation to the true solution than for the Anderson-Falicov-Kimball model, where the DMFT result is essentially temperature independent.

\subsection{3d Results}

Our main interest for the Anderson-Hubbard model lies in the 3d case. Here, we want to take a closer look at phase transitions. These are the antiferromagnetic transition, Mott transition and Anderson localization.

First, for the antiferromagnetic transition we look at the leading eigenvalue of $M=\tilde{V}_{m} \bar{\chi}_{0}$, which determines where the sum over the ladder diagrams for the antiferromagnetic susceptibility diverge. We compare results obtained from DMFT, 2nd order dual fermions and dual fermions with FLEX.

Second, we address the Mott metal-insulator transition. At high temperatures one observes a crossover, whereas for lower temperatures the transition is of first order. This leads to a hysteresis for the double occupancy $D$ as a function of $U$. We investigate the effect of disorder and temperature on the hysteresis.

Third, for Anderson localization, we have two quantities we look at. These are $\operatorname{Im} \delta G$ and the conductivity. $\operatorname{Im} \delta G$ is only applicable for binary disorder, because box disorder will not open a gap in the spectrum. As we cannot detect localized states from the arithmetically averaged density of states, we have to go beyond single-particle quantities for box disorder. We will use the conductivity, which is a defining quantity for an insulator. Therefore, it is supposed to work for both binary and box disorder.

In this chapter vertex corrections for the conductivity have been obtained only for the disorder component. In the region of the metal-bad-metal transition due to interactions the vertex corrections become very noisy and the noise translates to the conductivity, thus making calculations too costly for our purpose. Additionally, we suspect that the vertex correction do not fulfill the Ward identities [139], which relate the irreducible vertex with the self-energy. Instead, we relate the self-energy to the full vertex via the Schwinger-Dyson equation. The reason that we keep the vertex correction from disorder is that eq. 4.95 was originally introduced for disordered systems and it worked well in [40].

\subsubsection{Antiferromagnetic Transition}

The 3d Hubbard model has an antiferromagnetic phase. We want to map out this phase and investigate how this region changes upon introducing disorder. The natural way to determine this phase transition is to look at the antiferromagnetic susceptibility and where it diverges. In the dual fermion formalism, the antiferromagnetic susceptibility can be calculated from the dual fermion FLEX vertex ladder. The antiferromagnetic susceptibility will diverge if the dual fermion vertex ladder 


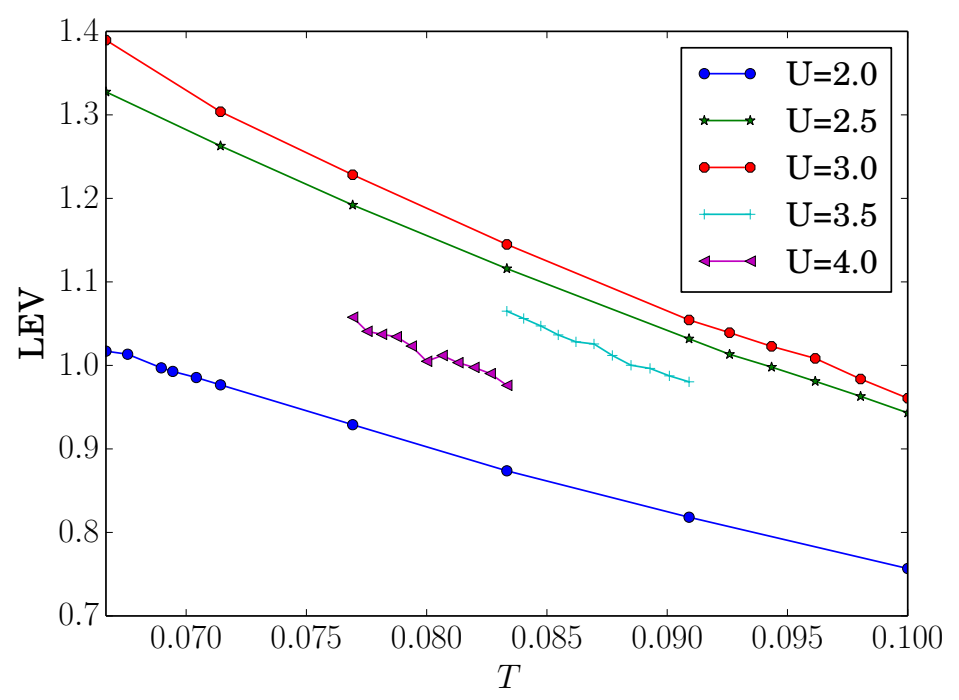

Figure 7.4: The LEV for the magnetic channel as a function of temperature for $V / W=1 / 3$ and various values of $U$. To extract the transition temperature we make a linear extrapolation to the data around $\mathrm{LEV}=1$.

diverges in the magnetic channel. This is equivalent to a leading eigenvalue (LEV) of 1 of the matrix $M=\tilde{V}_{m} \bar{\chi}_{0}$. Therefore, we limit ourselves to determining the LEV of $M$.

To this end, calculations are performed around the Néel temperature $T_{N}$. In cases of the second order calculation and DMFT a linear fit is used to determine the transition temperature. The change of the LEV for the second order dual fermion calculation with temperature is shown in fig. 7.4.

The FLEX calculation is a bit more involved as it becomes unstable for $\mid T-$ $T_{N} \mid<\delta$ for some finite value of $\delta$. To stabilize the calculation we check the leading eigenvalue in every step of the dual fermion calculation. If it exceeds 0.999 we set it to 0.999 . This trick allows to perform stable calculations at lower temperatures and to obtain fully self-consistent solutions even if in-between results may have a leading eigenvalue greater than one. Again, a linear extrapolation is used just between the two data points around LEV $=1$. There is some arbitrariness, though, as one could just as well use the last data point with LEV $<1$ as an upper bound and the value for LEV $>1$ is of course not very meaningful.

We see that $T_{N}$ first increases with interaction strength $U$ and then is reduced again. We did this for multiple values of $V$ and the results for binary disorder are shown in fig. 7.5. Disorder suppresses the antiferromagnetic phase for small values of $U$ and for large values of $U$ it increases $T_{N}$. The results in fig. 7.5 agree qualitatively with the findings for the infinite-dimensional Anderson-Hubbard model in reference [24]. Dual fermions decrease $T_{N}$ compared to DMFT and this effect is even stronger 


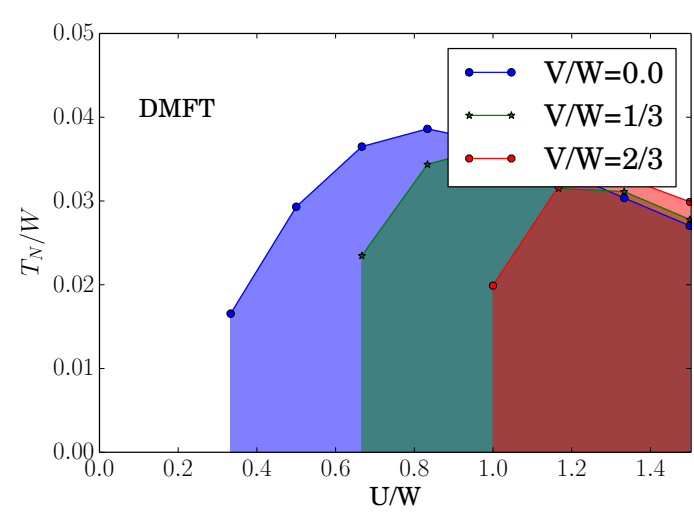

(a) DMFT

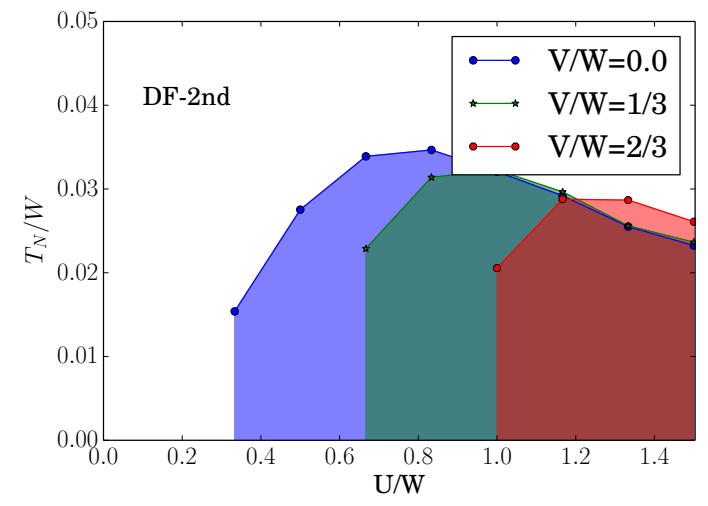

(b) DF-2nd

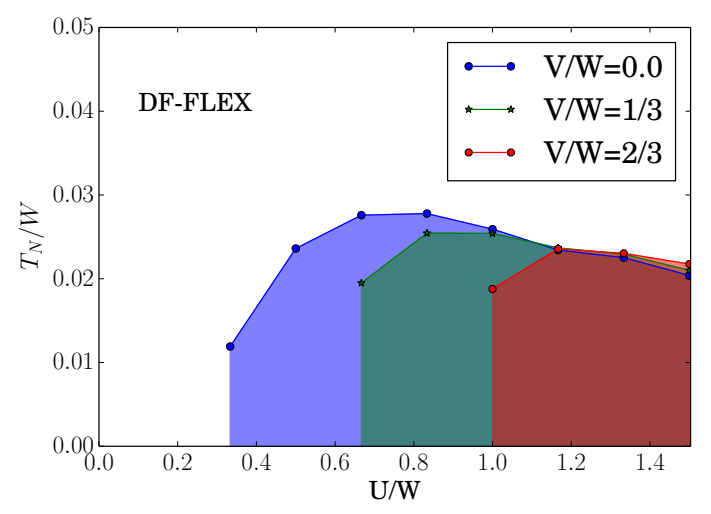

(c) DF-FLEX

Figure 7.5: The phase diagram on the $U T$ plane for various values of $V$ for binary disorder. In (a) the result for the DMFT calculation is shown, (b) shows the 2nd order dual fermion result and (c) shows the dual fermion FLEX result. All three methods agree qualitatively. Disorder suppresses antiferromagnetism for small values of $U$ and enhances it for large values of $U$. The effect of non-local correlations is to reduce the transition temperature for intermediate and large values of $U$. This effect is stronger for the FLEX calculation than for the 2nd order dual fermion results. 
for the FLEX approximation. DMFT as a mean-field method overestimates the antiferromagnetic phase. This is because mean-field methods interpret short-range antiferromagnetic correlations as long-range correlations. Dual fermions at least partially overcome this pathology.

For the clean system in the large- $\mathrm{U}$ limit for the 2 nd order calculation the phase boundary approaches a Weiss mean field solution for the Heisenberg-model. It is possible, that the FLEX solution approaches the exact solution for the Heisenbergmodel[105, 140]. Recent results [141] indicate that this is not the case for the value of $T_{c}$ but it has been found that the critical exponent of the inverse antiferromagnetic susceptibility for large values of $U$ takes on the value of the Heisenberg model. Hence, FLEX provides a qualitative improvement over DMFT and second order dual fermions.

In [24] the effect of disorder on the antiferromagnetic phase is explained physically. For small $U$ disorder reduces antiferromagnetic order. This is because disorder reduces the long-range order of the lattice, which in turn affects the long-range order of the electrons.

To understand how disorder promotes antiferromagnetism it is useful to look at two neighboring sites that are occupied by one electron each, both of different spins. For large $U$ and $U \gg V$ virtual hopping between the two sites with energy difference $\Delta V$ leads to a reduction of energy $J_{1}=-t^{2} /(U-\Delta V)$ for virtual hopping from site 1 to site 2 and $J_{2}=-t /(U+\Delta V)$ for the other direction. The effective spin coupling is

$$
J=J_{1}+J_{2}=\frac{2 t^{2}}{U}\left[1+\left(\frac{\Delta V}{U}\right)^{2}\right] .
$$

If one assumes that $T_{N} \propto\langle J(\Delta V)\rangle_{\text {dis }}$, it follows with $\Delta V=V_{1}-V_{2}$ that

$$
\frac{T_{N}(U, V)}{T_{N}(U, 0)}=\int d V_{1} \int d V_{2} J\left(V_{1}-V_{2}\right) p\left(V_{1}\right) p\left(V_{2}\right)=1+\lambda\left(\frac{V}{U}\right)^{2}
$$

where $\lambda$ is a parameter that depends on the disorder distribution. This shows that weak disorder increases $T_{N}$ for large $U$ and $U \gg V$ and it qualitatively explains the phase diagram that we observe. The DMFT result does indeed show an increase from $2.7 \%$ to $10 \%$ for the relative increase of $T_{N}$ when $V$ is doubled. We do not observe this for the dual fermion results. The results show some noise for large values of $U$ and the relative change is very small such that the quantitative agreement is not very good. Also, $U \gg V$ is not fulfilled, which may explain why we cannot see this behavior.

\subsubsection{Mott Metal-Insulator Transition}

A natural question to ask is how disorder affects the Mott metal-insulator transition. To address this question we investigate the change in the hysteresis of the double oc- 

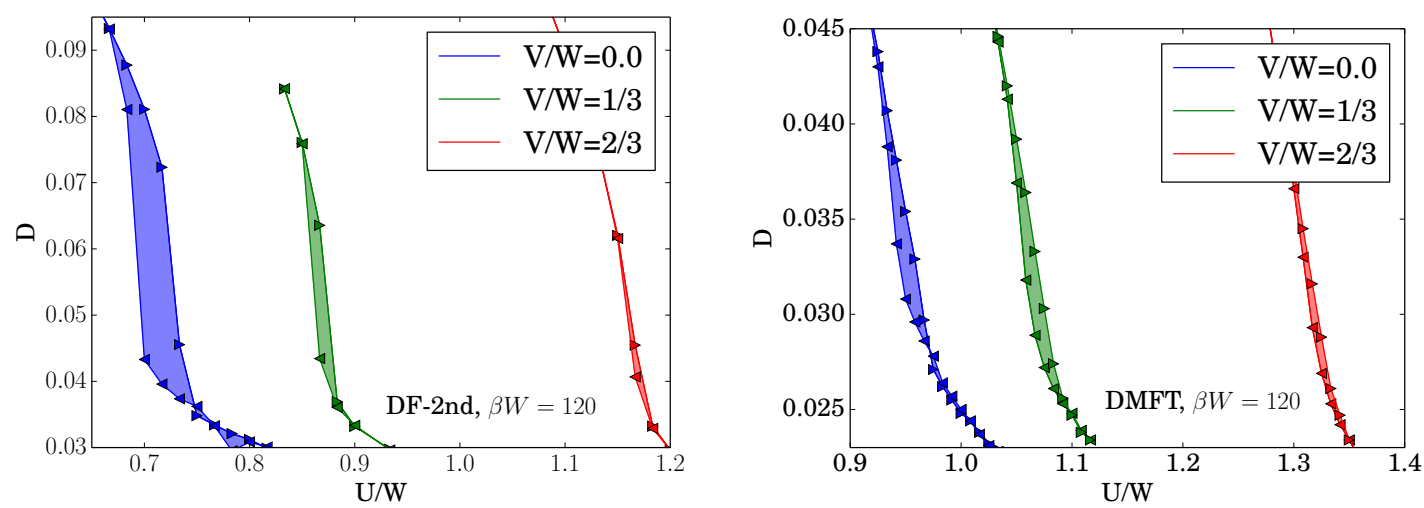

Figure 7.6: Hysteresis at $\beta W=120$ for binary disorder and various values of $V$. On the left, the 2nd order dual fermion result is shown, the right figure shows the DMFT result. In both cases disorder shifts the hysteresis to larger values of $U$ and reduces the area of the hysteresis. The effect of non-local correlations is to move the hysteresis to smaller values of $U$ and increase the area of the hysteresis. Note the different scaling of the $D$ axis in both figures to see the increase in the area for the green and red curves. (DMFT data by S.-X. Yang)

cupancy $D$. As the Mott transition is covered by the the antiferromagnetic phase we can only use the second order dual fermion approach. Figure 7.6 shows the hysteresis of the double occupancy for various values of $V$. With increasing $V$ the hysteresis is shifted to larger values of $U$, the area and width of the hysteresis are reduced. This is true both for the dual fermion results as well as for the DMFT results. The effect of the dual fermions is to reduce the lower and upper critical interaction strengths $U_{c_{1}}$ and $U_{c_{2}}$ while increasing the area of the hysteresis compared to DMFT. The reduction of $U_{c}$ comes from short-ranged antiferromagnetic correlations [142] that are present in the system even if we do not allow for antiferromagnetic ordering.

The qualitative agreement between dual fermions and DMFT changes when the temperature dependence of the hysteresis is investigated. Figure 7.7 presents the temperature dependence of the hysteresis for the clean system obtained from both dual fermions and DMFT. For DMFT decreasing temperature leads to an increase in $U_{c_{1}}$ and $U_{c_{2}}$, while in case of dual fermions, a decrease for the lower critical interaction strength $U_{c_{1}}$ can be observed instead. The effect on the upper critical interaction strength $U_{c_{2}}$ is unclear from fig. 7.7, as the data is too noisy to extract an unambiguous result. In fig. 7.8 we show the hysteresis for $V / W=1 / 6$ and $V / W=1 / 3$. Weak disorder does not change the reduction of $U_{c_{1}}$ with decreasing temperature but it is now visible that decreasing temperature increases $U_{c_{2}}$.

This qualitative change demonstrates that even in $3 \mathrm{~d}$ non-local correlations are important for a qualitatively correct physical picture. The reduced area and possible vanishing of the hysteresis indicate that disorder changes the order of the transition. 

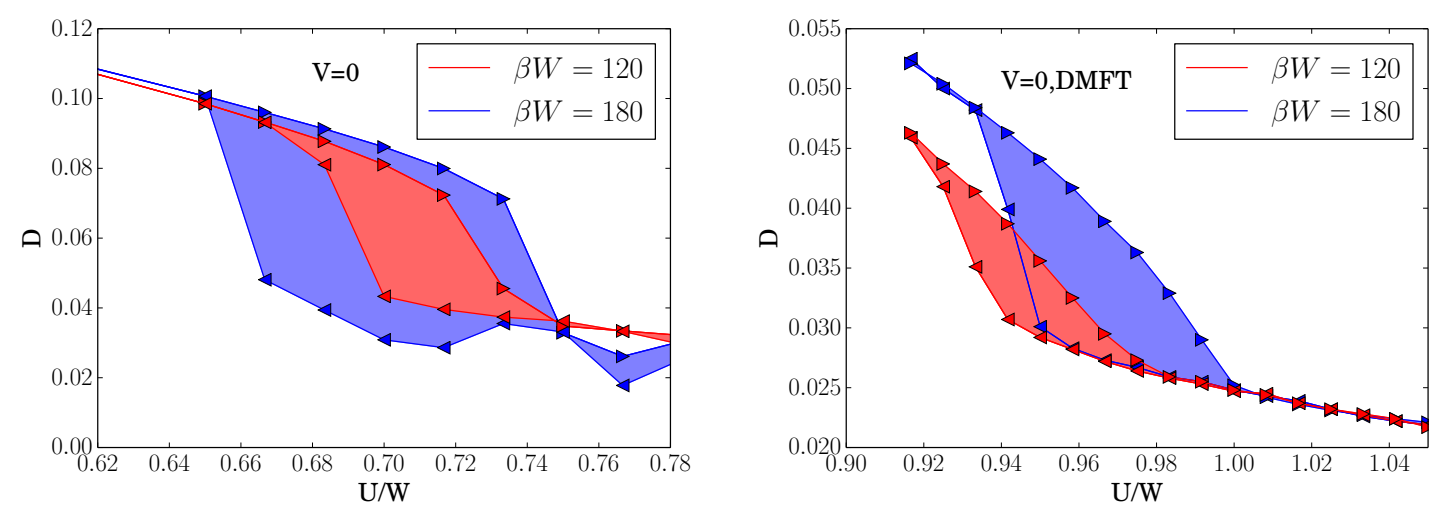

Figure 7.7: The dual fermion result on the left for the hysteresis shows a decreasing $U_{c_{1}}$ with decreasing temperature. This is in contrast with the DMFT result on the right, where $U_{c_{1}}$ increases with decreasing temperature. (DMFT data by S.-X. Yang)
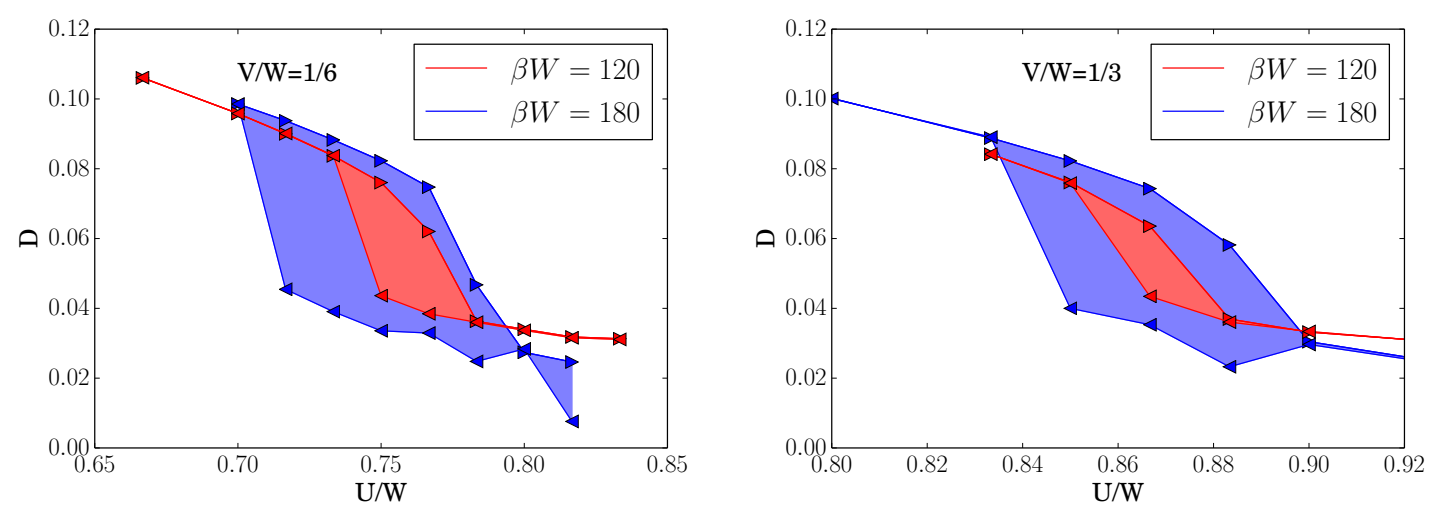

Figure 7.8: Double occupancy for $V / W=1 / 6$ and $V / W=1 / 3$. The qualitative picture from fig. 7.7 does not change with disorder. 


\subsubsection{Anderson Localization and Phase Diagram}

In this section we take a look at Anderson localization and the possibilities of its detection. In the following second order dual fermions is used.

To study the Anderson transition we look at $\operatorname{Im} \delta G$ and the conductivity on the $U V$-plane. $\operatorname{Im} \delta G$ is shown in fig. 7.9a for binary disorder and in fig. 7.10a for box disorder. If we use the $\operatorname{sign}$ of $\operatorname{Im} \delta G$ to determine the phase boundary between metal and bad metal, we find traces of localization for both large values of $U$ and $V$ for binary disorder. The shape of the metallic region is in agreement with [143], but we obtain smaller critical values for $U$ and $V$.

For box disorder, we find that the criterion only works for large values of $U$, but fails on the $V$-axis. Still, contour lines for $\operatorname{Im} \delta G>0$ allow to guess the shape of the metallic region. To confirm this guess we take a look at the conductivity. We can see from figs. $7.9 \mathrm{~b}$ and $7.10 \mathrm{~b}$ that the conductivity is strongly reduced both for binary as well as for box disorder for large values of $U$ and $V$, indicating Anderson localization. The general shape of the contour lines is the same for both the conductivity as well as for $\operatorname{Im} \delta G$. This confirms our guess from above, that the contour lines of $\operatorname{Im} \delta G$ reveal information about the shape of the metallic region. We fix the phase boundary by the value of the conductivity $\sigma_{d c}=0.04$ at $U_{c}$, where $\operatorname{Im} \delta G\left(U_{c}\right)=0$.

To test the accuracy of our approach, we compare with results obtained within the typical medium theory (TMT) [86]. TMT estimates the critical $V$ for binary disorder as $V_{A} \approx 0.4 W$ and for box disorder as $V_{A} \approx 1.1 W$ [144 at $T=0$. Typical medium DCA (TMDCA) increases the critical values to $V_{A} \approx 0.46 \mathrm{~W}$ for binary disorder and $V_{A} \approx 1.4$ for box disorder [144]. Dual fermions give $V_{A} \approx 0.4 W$ at $\beta W=60$ for binary disorder and $V_{A} \approx 1.0 \mathrm{~W}$ at $\beta W=60$ for box disorder. For binary disorder, our result agrees with TMT and is relatively close to the TMDCA result. For box disorder, our result is close to the TMT result but deviates significantly from the TMDCA result.

The reason that our results show better agreement for binary disorder is probably due to the fact that binary disorder opens a gap in the spectrum and non-selfaveraging effects thus play less of a crucial role. This is underlined by the fact that our estimate for $V_{A}$ from $\operatorname{Im} \delta G$ is $V_{A} \approx 0.45$, which is much closer to the TMDCA solution.

Possible improvements with our approach could be made by reducing the temperature and using a better approximation for the conductivity. Small changes in the value of the conductivity for the metal-bad-metal transition can change the critical disorder strength considerably. Nevertheless, we want to emphasize that the arithmetic disorder-average is not appropriate for an accurate treatment of Anderson localization as this is a non-self-averaging effect. Hence, we had no reasonable hope from the start to obtain a close-to-exact unambiguous value from our approach.

We conclude that dual fermions can be used to map out a finite-temperature phase diagram on the $U V$-plane including the Anderson insulator. Our results are 


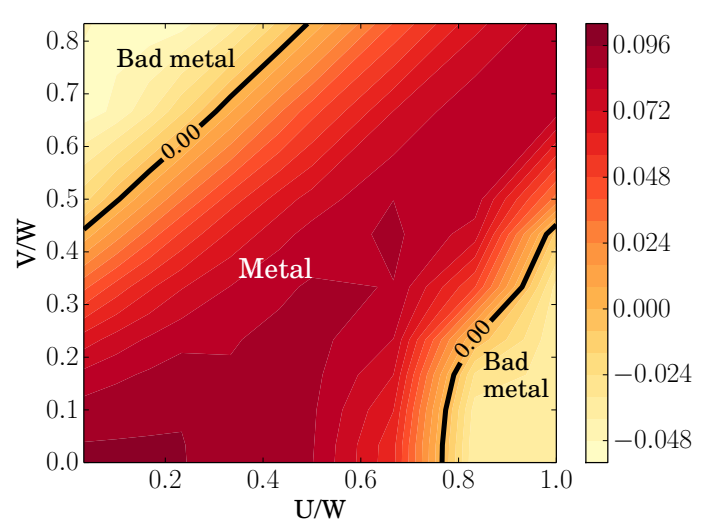

(a)

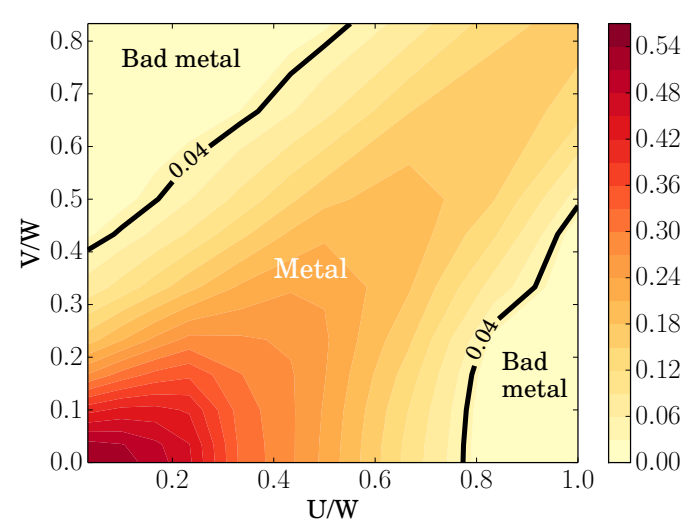

(b)

Figure 7.9: (a) $\operatorname{Im} \delta G$ and (b) the conductivity per spin as function of $U$ and $V$ for $\beta W=$ 60 . For both quantities we find the same general shape of the phase diagram. For such a high temperature, the elevated region can be interpreted as a metal whereas the lower region as a bad metal.

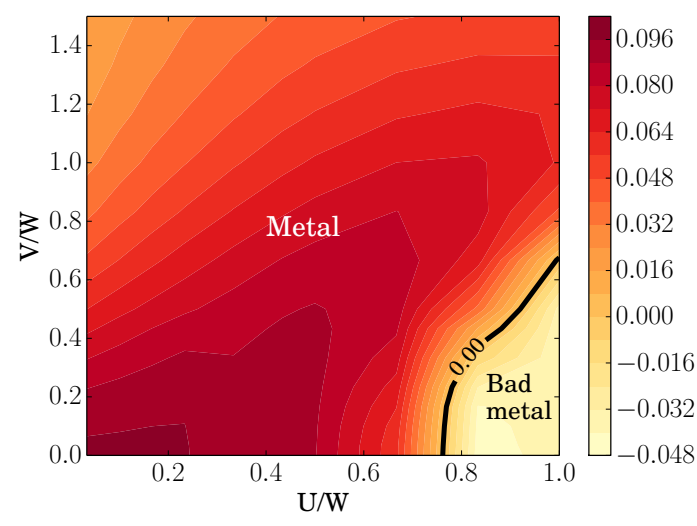

(a)

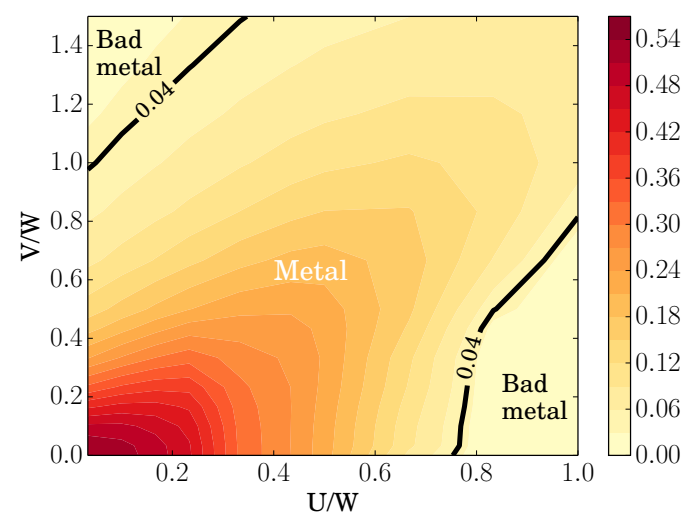

(b)

Figure 7.10: (a) $\operatorname{Im} \delta G$ and (b) the conductivity per spin as function of $U$ and $V$ for $\beta W=$ 60. In this case, $\operatorname{Im} \delta G$ and the conductivity show similar behavior close to the clean system. For stronger disorder the difference is quite noticeable because for box disorder $\operatorname{Im} \delta G$ does not show any signs of Anderson localization. 
in qualitative agreement with the phase diagram in [137, 145]. However, the critical disorder strength $V_{A}$ does not match the TMT and TMDCA results. For binary disorder, the estimate is relatively close to the numerical solution of [144]. Using the conductivity worsens the result in this case.

For box disorder, $V_{A}$ cannot be estimated from $\operatorname{Im} \delta G$. Our estimate from the conductivity is too small, but improved results for the conductivity on the $U$ axis may help to improve the estimate for $V_{A}$ and for the overall phase diagram.

If precise values for the critical disorder strength are needed, typical medium based methods may provide better results that can be used to obtain better estimates for the defining conductivity for the metal-insulator or metal-bad-metal transition.

\subsection{Summary}

In this chapter we applied the dual fermion formalism to the Anderson-Hubbard model. We started with a $1 \mathrm{~d}$ system in section 7.1, where the dual fermion approach was compared to DCA. Both methods gave qualitatively comparable results for the non-local corrections to DMFT. The quantitative agreement depends on the approximation used to solve the dual fermion problem. The FLEX approximation tends to provide better results if it is applicable.

We continued with the $3 \mathrm{~d}$ system in section 7.2 and looked at various phase transitions. In section 7.2.1 we investigated the effect on the antiferromagnetic transition. From [105] it is known, that dual fermions are capable of giving good approximate results for the transition temperature. The addition of weak disorder suppresses antiferromagnetism for small values of $U$ and enhances it for large values. This result was know from DMFT [24], dual fermions provide a quantitative improvement and possibly a qualitative improvement for the large $U$ limit. Unfortunately we were not able to study this limit as the computational cost becomes prohibitive.

Next, we investigated the effect of disorder on the Mott metal-insulator transition. To this end, we calculated the hysteresis of the double occupancy. The results show that disorder moves the critical values $U_{c_{1}}$ and $U_{c_{2}}$ to larger values and reduces the area of the hysteresis, indicating that for strong disorder the nature of the phase transition changes, possibly to a crossover. The effect of dual fermions is two-fold. For fixed temperature both $U_{c_{1}}$ and $U_{c_{2}}$ are decreased and the area of the hysteresis increases, but otherwise the qualitative picture stays the same.

A qualitative change appeared when the temperature dependence was investigated. DMFT predicts that $U_{c_{1}}$ and $U_{c_{2}}$ increase with decreasing temperature. Our dual fermion results show that $U_{c_{1}}$ decreases with decreasing temperature, which is qualitatively different from the DMFT result. At least in the case of disorder $U_{c_{2}}$ increases with decreasing temperature, which indicates that the upper critical value of $U$ is governed by mean-field behavior.

Finally, the Anderson transition was addressed in section 7.2.3. Although the dual 
fermions do not distinguish between localized and extended states, we were able to determine values for $V_{A}$ for binary and box disorder. In the case of box disorder, the results for $V_{A}$ do not agree very well with the results for $V_{A}$ from typical medium based methods, which have proved to be good approximations to the exact solution at $T=0$. This indicates that dual fermions have trouble dealing with non-selfaveraging effects. Additionally, we calculated the phase diagram on the $U V$ plane, which agrees qualitatively with the results of [143, 145].

We conclude that dual fermions add non-trivial modifications to the DMFT results. Quite often these are of a quantitative nature, but when non-local correlations are particularly important the changes can be qualitative as well, as we saw for the temperature dependence of the hysteresis. 



\section{Summary and Perspective}

The main goal of this thesis was the development of a numerical method that treats disorder and interaction on equal footing and goes beyond the dynamical mean-field approximation. To this end we started from the dual fermion approach which had been developed for interacting systems and which had been applied to disordered systems. We combined both approaches to treat disordered interacting systems.

Disorder was modeled with a local random potential that did not affect the hopping matrix elements. To obtain answers for macroscopic systems, i.e. systems which are self-averaging, we employed disorder-averaging.

In the derivation of the dual fermion formalism, the replica trick was used to perform the disorder-average on powers of the partition function $Z$ instead of the free energy $F=-T \ln Z$. As a result, the disorder-average became part of the impurity problem for the dual fermions and no disorder-average had to be done for the dual degrees of freedom. The replica trick introduced restrictions for the dual diagrams, thereby eliminating excitations for the dual degrees of freedom that are unphysical for the real degrees of freedom.

First, the dual fermion formalism was applied to the Anderson-Falicov-Kimball model. It is a good test ground, because the impurity problem for the dual fermion approach is trivial, but the model itself has non-trivial features like long-range order. The simplicity of the impurity problem manifests itself in the two-particle vertex function that only depends on two frequencies instead of three, as is the case for the Anderson-Hubbard model. Also, the DMFT solution for the single-particle Green function is essentially temperature independent. As our DCA calculations showed in $1 \mathrm{~d}$, this is not the correct answer but an artifact from ignoring the non-local correlations. We found that the dual fermion approach is able to reintroduce the correct temperature dependence.

For the 2d Anderson-Falicov-Kimball model we calculated the phase diagram on the $U V$ plane at $T=0.025 \mathrm{~W}$, where $U$ parametrizes the local Coulomb repulsion and $V$ the disorder strength. We found a metal-bad-metal transition for large values of $U$ and for large values of $V$, albeit the shape of the metallic region does not coincide with results obtained by other groups [41, 136]. We include non-local correlations whereas [41, 136] use geometric averaging, which is better suited to observe Anderson localization. It is thus hard to tell which result is correct. Possibly TMDCA [144] could provide an answer. The case of strong disorder and weak interaction poses the main challenge for the dual fermion approach as the physics becomes non-self-averaging. 
The driving force of the metal-bad-metal transition for weak disorder and strong Coulomb interactions is the formation of a charge density wave, which we learned from the leading eigenvalue of the Bethe-Salpeter equation. This is in accordance with reference [136, 146, 62]. On a bipartite lattice for interaction and weak disorder the ground state of the Anderson-Falicov-Kimball model is a checkerboard ordering of the $c$ and $f$ electrons. For the Anderson-Falicov-Kimball model results were shown exclusively for box disorder.

Second, the dual fermion approach was applied to the Anderson-Hubbard model, which is our model of interest. It has true quantum correlations, i. e. the two-particle vertex has the full dependence on three frequencies. Unless otherwise stated, all results were obtained for binary disorder. Again, we started with a comparison with DCA in 1d. The agreement is better than for the Anderson-Falicov-Kimball model, which might be due to the fact, that DMFT, which acts as a starting point for the dual fermion approach, provides a better, temperature dependent solution for the lattice problem.

The comparison with DCA is followed by results for the 3d system, which shows a variety of phase transitions. Emphasis was put on those phase transitions. We started with the phase diagram on the UT plane and distinguished two phases, namely paramagnetic metal and antiferromagnetic insulator. Results were shown for different values of the disorder strength $V$. DMFT, 2nd order dual fermions and dual fermion FLEX were used to obtain the phase diagram. All three methods gave the same shape of the phases and the effects of disorder were the same as well, specifically the reduction of antiferromagnetism for weak interaction and the enhancement of antiferromagnetism for strong interactions. In comparison with DMFT, 2nd order dual fermions reduced the critical temperature and dual fermion FLEX even more so. This was known for the clean system [105], and is assumed to be the correct behavior, based on DCA and quantum Monte Carlo studies [147, 140].

We continued with the Mott transition. To this end, we calculated the hysteresis of the double occupancy, which was approximated by the double occupancy of the impurity problem. As the Mott transition is concealed by the antiferromagnetic phase in the three dimensional unfrustrated system, the leading eigenvalue of the Bethe-Salpeter equation is greater than one, thus dual fermion FLEX could not be used. Therefore, we only obtained results for 2nd order dual fermions and DMFT.

At equal temperature, DMFT and dual fermions showed the same qualitative behavior. Disorder increases the critical interaction strength and reduces the area of the hysteresis. Dual fermions lower the critical interaction strength and increase the area of the hysteresis compared to DMFT. This is due to short-range antiferromagnetic correlations that are not present in DMFT. It would be interesting to extend the calculation to the strongly frustrated system and see the effect of FLEX on the Mott transition.

A qualitative difference between dual fermions and DMFT was found when we 
investigated the temperature dependence of the hysteresis. In DMFT the upper and lower critical value of the interaction strength are increased with decreasing temperature. With dual fermions we found that at least the lower critical interaction strength decreases. Our data was too noisy to extract unambiguous results for the upper critical value for the clean system. Upon the introduction of disorder the upper critical value is clearly increased with decreasing temperature, thus showing mean-field behavior. We expect this for the lower critical value as well when disorder is strong enough, but we were not able to obtain data at sufficiently strong disorder as increasing disorder meant calculations at large values of $U$, unaccessible to us.

Lastly, Anderson localization was investigated. The main focus was placed on the question of how to detect it and how it compares with established methods like typical-medium-theory or the newer and very promising TMDCA [148]. For detection, we used the imaginary part of the local Green function, namely the difference between the lowest two frequencies, which determines whether the imaginary part has a minimum or not. This criterion only worked for binary disorder. Therefore, we calculated the conductivity as well, which allowed to determine a phase boundary. Our results for the shape of the metallic region agree with results obtained previously [143]. Unfortunately, our estimate for the critical disorder strength for box disorder does not match results obtained within typical medium theory and TMDCA. This is not completely unexpected, as Anderson localization is a non-self-averaging effect and thus the disorder-average we used is not suited to detect it.

Our results from both the Anderson-Falicov-Kimball model and the AndersonHubbard model suggest that dual fermions add non-trivial corrections to the DMFT result and do so on a level that is qualitatively and oftentimes quantitatively comparable with DCA. Hence, we believe that the formalism developed in this thesis can help further grow the understanding of disordered interacting systems. For example, it is an interesting topic, how disorder influences superconductivity. Is it possible, that disorder enhances superconductivity in certain cases as it does with antiferromagnetism and can this be quantified?

A particularly interesting case consists in the application to real materials. For this purpose, the dual fermion formalism has to be combined with first-principle methods like density functional theory (DFT) [149, 150]. In order to include quenched disorder, it has to be worked out, whether it is possible to start from a DFT solution for a clean system and add disorder only at the impurity and dual fermion level or whether disorder has to be treated already on the DFT level. In the latter case, the interfacing between the DFT part and the dual fermion part has to be addressed.

Finally, we want to propose a further extension of the dual fermion formalism. In [151] experiments on layered $f$-electron systems are presented. These layered systems have been investigated by means of inhomogeneous or real-space DMFT (rDMFT) [152]. To this end, the dual fermion formalism for the periodic Anderson model could be used to solve individual layers. The coupling between different layers 
could be treated analogously to rDMFT. Additionally, such a scheme could be used to investigate thin film superconductors as were mentioned in section 2.2 .

This proposal shows that there is still a wide range of possible applications of the dual fermion approach, be it for interacting, disordered or interacting disordered systems. 
Appendices 



\section{A The Sign Problem in Quantum Monte Carlo Simulations}

The following discussion is based on the explanation of the sign problem in [25]. Quantum-Monte Carlo methods are very powerful tools if applicable. Although many algorithm scale at least with $\left(U N_{c} \beta\right)^{3}$, calculations at lower temperatures and larger cluster sizes will be achievable as long as computers become faster. However, if it is impossible to retain positive weights for all Monte Carlo configurations for a given system, Quantum-Monte Carlo methods hit a hard wall. In the following we will discuss why that is.

We start from the partition function

$$
Z=\operatorname{Tr} e^{-\beta H}=\sum_{C} w_{C}
$$

$\sum_{C}$ denotes a sum over configurations as discussed is chapter 5 and $w_{C}$ are the corresponding contributions to the partition function. The form of $w_{C}$ depends on the algorithm that is used.

Now we want to use Monte Carlo methods to sample the configurations $C$. This requires that $w_{C} \geq 0$. We will assume that this is not the case for our problem and that there is no known trick to achieve this. In that case, we can write

$$
Z=\sum_{C} w_{C}=\sum_{C} \operatorname{sgn}\left(w_{C}\right)\left|w_{C}\right|
$$

and we can use $\left|w_{C}\right|$ as weights for the Monte Carlo simulation. For the expectation value of an observable $O$ we have

$$
\langle O\rangle=\frac{1}{Z} \sum_{C} O_{C} w_{C}=\sum_{C} \operatorname{sgn}\left(w_{C}\right) O_{C}\left|w_{C}\right|=\frac{\langle\operatorname{sgn} O\rangle_{|w|}}{\langle\operatorname{sgn}\rangle_{|w|}} .
$$

Here, $O_{C}$ is the value of $O$ in configuration $C$ and $\langle\ldots\rangle_{|w|}$ denotes an expectation value with respect to $\left|w_{C}\right|$.

Equation A.3 shows how to deal with negative weights. At this point there is no reason to expect that there might be a problem beyond numerical accuracy - and such a problem can be solved by trading time for accuracy by using special purpose libraries. 
We proceed by taking a look at the average sign

$$
\langle\operatorname{sgn}\rangle_{|w|}=\frac{\sum_{C} \operatorname{sgn}\left(w_{C}\right)\left|w_{C}\right|}{\sum_{C}\left|w_{C}\right|} .
$$

The nominator is just the partition function of our system, the denominator is a partition function for a system with only positive weights. According to thermodynamics, the partition function can be written in terms of the free energy $F=-k_{B} T \ln Z$ where $1 / \beta=k_{B} T . k_{B}$ is the Boltzmann constant and $T$ temperature. It follows that

$$
\langle\operatorname{sgn}\rangle_{|w|}=e^{-\beta \Delta F} .
$$

We know that $\langle\operatorname{sgn}\rangle_{|w|} \leq 1$, therefore $\Delta F \geq 0$. This means the average sign becomes exponentially smaller for increasing $\beta$ and for increasing $\Delta F$. When we approach the thermodynamic limit, i. e. all relevant correlations are included in the cluster for the Monte Carlo simulation, we will have $\Delta F \propto N_{c}$. This means the sign scales exponentially in $\beta$ and $N_{C}$. This is still no unsolvable problem, all we need is the corresponding numerical accuracy. This costs time but in principle it should be possible.

Next, we look at the variance of the sign:

$$
\operatorname{Var}(\operatorname{sgn})=\left\langle\operatorname{sgn}^{2}\right\rangle_{\left|w_{C}\right|}-\langle\operatorname{sgn}\rangle_{\left|w_{C}\right|}^{2}=1-e^{-2 \beta \Delta F}
$$

The Monte Carlo error of an observable $O$ after $N$ iterations is

$$
\Delta_{M C}(O) \propto \frac{\sqrt{\operatorname{Var}(O)}}{\sqrt{N}} .
$$

The relative error of the sign is

$$
\Delta \operatorname{sgn} \propto \frac{\sqrt{\operatorname{Var}(\operatorname{sgn}) / N}}{\langle\operatorname{sgn}\rangle_{\left|w_{C}\right|}} \approx \frac{e^{\beta \Delta F}}{\sqrt{N}}
$$

after $N$ Monte Carlo measurements. This means that in order to obtain the sign with reasonable accuracy the number of Monte Carlo measurements has to be increased exponentially. This is the reason the sign is a problem. The time needed to solve a given problem with the needed accuracy scales exponentially. 


\section{B Fast Matrix Updates for Quantum Monte Carlo}

For the ctQMC random walk it is necessary to calculate the ratio of two determinants. For arbitrary matrices the calculation of the determinant is of order $N^{3}$ and such would be the time to calculate the ratio. However, the smaller of our two matrices appears in the larger one. This can be expressed as [25]

$$
N^{(n+1)}=\left(\begin{array}{cc}
N^{(n)} & Q \\
R & S
\end{array}\right)
$$

where $N^{(n)}$ is a matrix of rank $n$. For the cases of importance in this work, $S$ is a number, $Q$ a column vector and $R$ a row vector. The inverse of $N^{(n+1)}$ is

$$
\left(N^{(n+1)}\right)^{-1}=\left(\begin{array}{cc}
\tilde{P} & \tilde{Q} \\
\tilde{R} & \tilde{S}
\end{array}\right) .
$$

A matrix that consists of four blocks can be decomposed as

$$
\left(\begin{array}{cc}
A & B \\
C & D
\end{array}\right)=\left(\begin{array}{ll}
A & 0 \\
C & \mathbb{1}
\end{array}\right) \cdot\left(\begin{array}{cc}
\mathbb{1} & A^{-1} B \\
0 & D-C A^{-1} B
\end{array}\right),
$$

which is verified by direct calculation. If one of the off-diagonal blocks is zero, the determinant is

$$
\operatorname{det}\left(\begin{array}{cc}
A & B \\
0 & D
\end{array}\right)=\operatorname{det} A \cdot \operatorname{det} D .
$$

The matrices $\tilde{S}, \tilde{Q}, \tilde{R}$ and $\tilde{P}$ are given by [131]

$$
\begin{aligned}
\tilde{S} & =\left(S-R\left(N^{(n)}\right)^{-1} Q\right)^{-1} \\
\tilde{Q} & =-\left(N^{(n)}\right)^{-1} Q \tilde{S} \\
\tilde{R} & =-\tilde{S} R\left(N^{(n)}\right)^{-1} \\
\tilde{P} & =\left(N^{(n)}\right)^{-1}+\left(N^{(n)}\right)^{-1} Q \tilde{S} R\left(N^{(n)}\right)^{-1} .
\end{aligned}
$$


Combining all this together, we arrive at

$$
\frac{\operatorname{det} N^{(n+1)}}{\operatorname{det} N^{(n)}}=\operatorname{det}\left(S-R\left(N^{(n)}\right)^{-1} Q\right)=\frac{1}{\operatorname{det} \widetilde{S}}
$$

for the matrix ratios. In the ctQMC algorithm $N^{-1}$ is stored, therefore eq. B.9 is particularly cheap for the removal of a vertex. It is only necessary to look up $\tilde{S}$, which is $O(1)$. For the addition of a vertex, matrix-matrix multiplication is necessary, which is $O\left(n^{2}\right)$. The same holds true for the update of the matrix. 


\section{Dual Fermion Mapping for the Anderson-Falicov-Kimball Model}

The derivation of the dual fermion approach for the Anderson-Falicov-Kimball model is similar to the Anderson-Hubbard model. Instead of spin up and down we have the $c$ - and $f$-degrees of freedom. Here, we closely follow the derivation that we published in [106]. In the following, the $f$-degrees of freedom will be denoted as $c^{f}$ so they are not confused with the dual degrees of freedom $f$. We start the derivation from the disorder-averaged Green function

$$
\left\{G_{k}\right\}=-\left.\frac{\delta}{\delta \eta_{k}}\left\{\ln Z^{v}\left[\eta_{k}\right]\right\}\right|_{\eta_{k}=0}
$$

following [106]. $\eta_{k}$ is a source field, $\{\ldots\}$ denotes a disorder-average and $X^{v}$ is the quantity $X$ in the disorder configuration $v$. The partition function $Z$ for the Anderson-Falicov-Kimball model reads

$$
Z^{v}\left[\eta_{k}\right]=\int D[c, \bar{c}] \int D\left[c^{f}, \bar{c}^{f}\right] e^{-S^{v}\left[\bar{c}, c ; \bar{c}^{f}, c^{f} ; \eta_{k}\right]}
$$

in a configuration $v$. The action for this configuration is

$$
S^{v}\left[\bar{c}, c ; \bar{c}^{f}, c^{f} ; \eta_{k}\right]=-\sum_{\omega, k, \sigma} \bar{c}_{\omega, k, \sigma}\left(\omega+\mu-\epsilon_{k}-\eta_{k}\right) c_{\omega, k, \sigma}+\sum_{i} S_{i}^{v}\left[\bar{c}_{i}, c_{i} ; \bar{c}_{i}^{f}, c_{i}^{f}\right]
$$

with

$$
S_{i}^{v}\left[\bar{c}_{i}, c_{i} ; \bar{c}_{i}^{f}, c_{i}^{f}\right]=\int_{0}^{\beta} d \tau v_{i} n_{i}(\tau)+\int_{0}^{\beta} d \tau U n_{i}^{c}(\tau) n_{i}^{f}(\tau) .
$$

We rewrite the action in terms of an impurity action

$$
S_{i}^{v, \operatorname{imp}}\left[\bar{c}_{i}, c_{i} ; \bar{c}_{i}^{f}, c_{i}^{f}\right]=-\sum_{\omega} \bar{c}_{\omega i}\left[(\omega+\mu)-\Delta_{\omega}\right] c_{\omega i}+S_{i}^{v}\left[\bar{c}_{i}, c_{i}, \bar{c}_{i}^{f}, c_{i}^{f}\right] .
$$

Here, we introduced a hybridization function $\Delta_{\omega}$. The action reads

$$
S^{v}\left[\bar{c}, c ; \bar{c}^{f}, c^{f}\right]=\sum_{i} S_{i}^{v, \operatorname{imp}}\left[\bar{c}_{i}, c_{i}\right]-\sum_{\omega, k, \sigma} \bar{c}_{\omega, k, \sigma}\left(\Delta_{\omega}-\epsilon_{k}\right) c_{\omega, k, \sigma}
$$


in terms of the impurity action $S_{i}^{v, \text { imp }}$. Note that the hybridization function drops out if we insert the impurity action.

Next, we apply the replica trick [81, 82]

$$
\langle\ln Z\rangle_{d i s}=\lim _{m \rightarrow 0} \frac{\left\langle Z^{m}\right\rangle_{d i s}-1}{m}
$$

and the Green function now reads

$$
\begin{aligned}
\left\langle G_{k}\right\rangle_{d i s} & =-\frac{\delta}{\delta \eta_{k}} \int \prod_{i} d v_{i} p\left(v_{i}\right) \ln Z^{i v} \\
& =-\lim _{m \rightarrow 0} \frac{1}{m} \frac{\delta}{\delta \eta_{k}} \int \prod_{i} d v_{i} p\left(v_{i}\right) \iint \prod_{\alpha} D\left[c_{\alpha}, \bar{c}_{\alpha}\right] D\left[c_{\alpha}^{f}, \bar{c}_{\alpha}^{f}\right] e^{S_{\alpha}^{v}}
\end{aligned}
$$

$\alpha$ is a replica index that goes from 1 to $m$.

Disorder can be integrated out [84] according to

$$
\int d v_{i} p\left(v_{i}\right) e^{-v_{i} \sum_{\alpha} \int d \tau n_{i}^{\alpha}(\tau)}=e^{W\left(\tilde{n}_{i}^{c}\right)} .
$$

This leads to an effective elastic interaction $W$ between replicas that reads

$$
\begin{aligned}
& W(\tilde{n})=\sum_{l=2}^{\infty} \frac{1}{l !}\left\langle v_{i}^{l}\right\rangle_{c} \tilde{n}^{l} . \\
& \tilde{n}=\sum_{\alpha} \int d \tau\left(n^{c}\right)_{i}^{\alpha}(\tau) .
\end{aligned}
$$

$\left\langle v_{i}^{l}\right\rangle_{c}$ denotes the $l$ th cumulant of the disorder distribution.

In the next step, the dual degrees of freedom are introduced via a HubbardStratonovich transformation

$$
e^{\bar{c}_{\omega k}^{\alpha} A_{\omega k}^{2} c_{\omega k}}=\frac{A_{\omega k}^{2}}{\lambda_{\omega}^{2}} \int D \bar{f} D f e^{-\lambda_{\omega}\left(\bar{c}_{\omega k}^{\alpha} f_{\omega k}^{\alpha}+\bar{f}_{\omega k}^{\alpha} c_{\omega k}^{\alpha}\right)-\frac{\lambda_{\omega}^{2}}{A_{\omega k}^{2}} \bar{f}_{\omega k}^{\alpha} f_{\omega k}^{\alpha}} .
$$

The $f$-numbers are Grassmann numbers, which follow fermionic commutation relations. For $A$ we obtain

$$
A_{\omega k}^{2}=\Delta_{\omega}-\epsilon_{k}-\eta_{\omega k}
$$

and $\lambda_{\omega}$ remains arbitrary as in the derivation for the Anderson-Hubbard model. After the Hubbard-Stratonovich transformation the Green function reads

$$
\begin{aligned}
G_{\omega k} & =-\lim _{m \rightarrow 0} \frac{1}{m} \frac{\delta}{\delta \eta_{\omega k}}\left(\prod_{\omega k} \frac{\Delta_{\omega}-\epsilon_{k}-\eta_{\omega k}}{\lambda_{\omega}^{2}}\right)^{m} \times \\
& \times\left.\int D \bar{f} D f e^{-\sum_{\omega k \alpha} \lambda_{\omega}^{2} \bar{f}_{\omega k}^{\alpha}\left(\Delta_{\omega}-\epsilon_{k}-\eta_{\omega k}\right)^{-1}} f_{\omega k}^{\alpha} \int D \bar{c} D c e^{-\sum_{i} S_{s i t e}^{i}}\right|_{\eta_{\omega k}=0} .
\end{aligned}
$$


The $c$ - and $c^{f}$-degrees of freedom both appear only locally and can be integrated out. Now, the local part of the action reads

$$
S_{\text {site }}^{i}=S_{\text {imp }}^{i}+\sum_{\omega \alpha} \lambda_{\omega}\left(\bar{c}_{\omega i}^{\alpha} f_{\omega i}^{\alpha}+\bar{f}_{\omega i}^{\alpha} c_{\omega i}^{\alpha}\right)
$$

with

$$
S_{\mathrm{imp}}^{i}=-\sum_{\alpha} \sum_{\omega} \bar{c}_{\omega i}^{\alpha}\left[(\omega+\mu)-\Delta_{\omega}\right] c_{\omega i}^{\alpha}-W\left(\tilde{n}_{i}\right)+\sum_{\alpha} \int_{0}^{\beta} d \tau U n_{i}^{c, \alpha}(\tau) n_{i}^{f, \alpha}(\tau) .
$$

Note that only the $c$-degrees of freedom couple to the $f$-degrees of freedom. This is because the $c^{f}$-degrees of freedom only appear locally in the Hamiltonian eq. 2.6 and are thus part of the impurity problem.

We can now write down the defining equation for the dual potential which reads

$$
\int \prod_{\alpha \omega} d \bar{c}^{\alpha} d c^{\alpha} d \bar{c}^{f, \alpha} d c^{f, \alpha} e^{-S_{s i t e}}=Z_{\mathrm{imp}} e^{-\sum_{\omega \alpha} \lambda_{\omega}^{2} g_{\mathrm{imp}}(\omega) \bar{f}_{\omega}^{\alpha} f_{\omega}^{\alpha}-V_{d}\left[\bar{f}^{\alpha}, f^{\beta}\right]}
$$

for the Anderson-Falicov-Kimball model. As an index, $\beta$ labels replicas.

The impurity partition function is

$$
Z_{\mathrm{imp}}=\int \prod_{\alpha \omega} d \bar{c}^{\alpha} d c^{\alpha} d \bar{c}^{f, \alpha} d c^{f, \alpha} e^{-S_{\mathrm{imp}}}
$$

At this point we choose $\lambda_{\omega}=g_{\omega}^{-1}$ and obtain

$$
V_{d}\left[\bar{f}^{\alpha}, f^{\beta}\right]=\frac{1}{2} V^{p, 0}\left(\omega, \omega^{\prime}\right) \bar{f}^{\alpha} \bar{f}^{\beta} f^{\beta} f^{\alpha}+\frac{1}{4} V_{\nu}^{p, 1}\left(\omega, \omega^{\prime}\right) \bar{f}^{\alpha} \bar{f}^{\alpha} f^{\alpha} f^{\alpha} .
$$

$g_{\omega}$ is the impurity Green function. This is analogous to the derivation of the dual potential for the Anderson-Hubbard model. Again, we restricted ourselves to the lowest order in the dual potential.

Finally, we can perform the derivative with respect to the source field $\eta_{k}$ and obtain

$$
G(\omega, k)=\left(\Delta_{\omega}-\epsilon_{k}\right)^{-1}+\frac{G_{d}}{g_{\omega}^{2}\left(\Delta_{\omega}-\epsilon_{k}\right)}
$$

for the real fermion Green function. The dual Green function $G_{d}$ is defined as

$$
G_{d}=-\lim _{m \rightarrow 0} \frac{1}{m} \sum_{\alpha^{\prime}}^{m} \int \prod_{\rho, \omega, k} d \bar{f}_{\omega, k}^{\rho} d f_{\omega, k}^{\rho} f^{\alpha^{\prime}} \bar{f}^{\alpha^{\prime}} e^{-\sum_{\omega k \alpha^{\prime}} S_{d 0}} e^{\sum_{i \alpha \beta \omega} V_{d}\left[\bar{f}^{\alpha}, f^{\beta}\right]} .
$$

The non-interacting action $S_{d 0}$ reads

$$
S_{d 0}=\bar{f}_{\omega k}^{\alpha}\left[\frac{\left(\Delta-\epsilon_{k}\right)^{-1}+g_{\omega}}{g_{\omega}^{2}}\right] f_{\omega k}^{\alpha}
$$


For non-interacting dual fermions this gives the DMFT result, which therefore appears as a zeroth order approximation in this formalism. 


\section{Two-Particle Green Functions}

The following definitions are taken from [153, 105]. The two-particle Green functions are defined via functional derivatives of the free energy as

$$
\chi^{p h}=\left.\frac{\delta^{4} F[\bar{K}, K]}{\delta K_{4} \delta \bar{K}_{3} \delta K_{2} \delta \bar{K}_{1}}\right|_{\bar{K}_{1}=K_{2}=\bar{K} 3=K 4=0}
$$

for the particle-hole channel. The particle-hole Green function reads

$$
\chi^{p h}\left(\tau_{1}, \tau_{2} ; \tau_{3}, \tau_{4}\right)=\left\langle T_{\tau} c\left(\tau_{1}\right) c^{\dagger}\left(\tau_{2}\right) c\left(\tau_{3}\right) c^{\dagger}\left(\tau_{4}\right)\right\rangle-\left\langle T_{\tau} c\left(\tau_{1}\right) c^{\dagger}\left(\tau_{2}\right)\right\rangle\left\langle T_{\tau} c\left(\tau_{3}\right) c^{\dagger}\left(\tau_{4}\right)\right\rangle .
$$

For the particle-particle channel the two-particle Green function is defined as

$$
\chi^{p p}=\left.\frac{\delta^{4} F[\bar{K}, K]}{\delta K_{4} \delta K_{3} \delta \bar{K}_{2} \delta \bar{K}_{1}}\right|_{\bar{K}_{1}=\bar{K}_{2}=K 3=K 4=0}
$$

and the particle-particle Green function reads

$$
\chi^{p p}\left(\tau_{1}, \tau_{2} ; \tau_{3}, \tau_{4}\right)=\left\langle T_{\tau} c\left(\tau_{1}\right) c\left(\tau_{2}\right) c^{\dagger}\left(\tau_{3}\right) c^{\dagger}\left(\tau_{4}\right)\right\rangle .
$$

For the particle-hole Green function the vacuum term is subtracted which follows from the chain rule. For the particle-particle channel the chain rule leads to $\langle c c\rangle\left\langle c^{\dagger} c^{\dagger}\right\rangle$ which is zero for all models in this thesis.

We obtain the Fourier transformed two-particle quantities for the particle-hole Green function via

$\chi^{p h}\left(\omega_{1}, \omega_{2} ; \omega_{3}, \omega_{4}\right)=\int_{0}^{\beta} d \tau_{1} \int_{0}^{\beta} d \tau_{2} \int_{0}^{\beta} d \tau_{3} \int_{0}^{\beta} d \tau_{4} \chi\left(\tau_{1}, \tau_{2} ; \tau_{3}, \tau_{4}\right) \mathrm{e}^{i\left(\omega_{1} \tau_{1}-\omega_{2} \tau_{2}+\omega_{3} \tau_{3}-\omega_{4} \tau_{4}\right)}$.

Time-translational invariance requires $\omega_{1}+\omega_{3}=\omega_{2}+\omega_{4}$. This means $\chi$ only depends on three frequencies, two fermionic ones and one bosonic one, which is the difference $\nu:=\omega_{1}-\omega_{2}=\omega_{4}-\omega_{3}$ of two fermionic frequencies. $\nu$ denotes the total energy of the particle-hole pair, 1(4) denotes the initial (final) electron, 2(3) the initial (final) hole. We use time-translational invariance to introduce the three frequency notation 105

$$
\chi^{p h}(\nu)_{\omega, \omega^{\prime}}:=\chi^{p h}\left(\omega+\nu, \omega ; \omega^{\prime}, \omega^{\prime}+\nu\right),
$$

which is shown in fig. D.1. The particle-particle Green function is Fourier trans- 


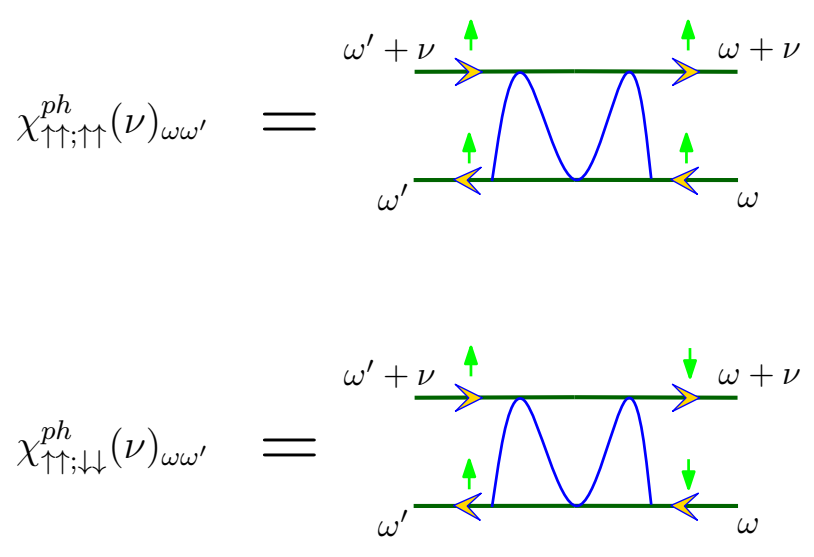

Figure D.1: The two particle-hole Green function shown here are all Green functions needed for an $\mathrm{SU}(2)$ symmetric system.

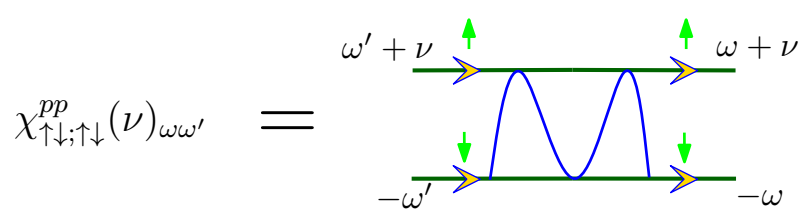

Figure D.2: In the particle-particle channel we need only the pairing channel Green function. We can construct all other particle-particle Green functions from it.

formed via

$\chi^{p p}\left(\omega_{1}, \omega_{2} ; \omega_{3}, \omega_{4}\right)=\int_{0}^{\beta} d \tau_{1} \int_{0}^{\beta} d \tau_{2} \int_{0}^{\beta} d \tau_{3} \int_{0}^{\beta} d \tau_{4} \chi\left(\tau_{1}, \tau_{2} ; \tau_{3}, \tau_{4}\right) \mathrm{e}^{i\left(\omega_{1} \tau_{1}+\omega_{2} \tau_{2}-\omega_{3} \tau_{3}-\omega_{4} \tau_{4}\right)}$.

In this case the bosonic frequency is defined as $\nu:=\omega_{1}+\omega_{2}=\omega_{4}+\omega_{3} .1$ and 2 are the initial electrons, 3 and 4 the final electrons. The three frequency notation is

$$
\chi^{p p}(\nu)_{\omega, \omega^{\prime}}:=\chi^{p p}\left(\omega+\nu,-\omega ;-\omega^{\prime}, \omega^{\prime}+\nu\right),
$$

which is illustrated in fig. D.2. The difference in sign for the frequencies in the particle-hole and particle-particle channel comes from the convention that a particle with frequency label $\omega$ carries the energy $\omega$ whereas a hole with the frequency label $\omega$ carries the energy $-\omega$. The total energy is the sum of the top and the bottom where a hole introduces an additional minus sign. If $\nu$ is to denote the total energy of the particle-hole (particle-particle) pair we obtain the notation as as shown above. 


\section{D.1 Channels}

For the two-particle Green function we have not only the particle-hole and particleparticle channel, but we have six different possible spin configurations as well. For the particle-hole channel these are $(\uparrow \uparrow ; \uparrow \uparrow),(\downarrow \downarrow ; \downarrow \downarrow),(\uparrow \uparrow ; \downarrow \downarrow),(\downarrow \downarrow ; \uparrow \uparrow),(\uparrow \downarrow ; \downarrow \uparrow)$ and $(\downarrow \uparrow ; \uparrow \downarrow)$. The first four have $s_{z}=0$ for the initial and final state, whereas the last two have $s_{z}=1$ and $s_{z}=-1$. A hole of spin up is effectively a down spin because the lack of an up spin leaves an uncompensated down spin.

We have to measure these channels using quantum Monte Carlo. It is possible to exploit symmetries to reduce the number of channels we have to measure down to two for the particle-hole channel. This reduces the memory needed to store the vertex function and the time needed to measure the particle-hole Green functions by a factor of three.

First we note that the last two configurations have a total spin $S=1$. There is one state missing for a triplet. We can create this state via

$$
\chi_{m^{0}}=\frac{1}{\sqrt{4}}\left(\chi_{\uparrow \uparrow ; \uparrow \uparrow}^{p h}+\chi_{\downarrow \downarrow ; \downarrow \downarrow}^{p h}+\chi_{\uparrow \uparrow ; \downarrow \downarrow}^{p h}+\chi_{\downarrow \downarrow ; \uparrow \uparrow}^{p h}\right)
$$

which defines the magnetic channel $m$ with $s_{z}=0$. For the magnetic channel the initial and final states have a total spin $S=1$. we can see this by symbolically writing

$$
\left.\frac{1}{\sqrt{4}}((\uparrow \uparrow ; \uparrow \uparrow)+(\downarrow \downarrow ; \downarrow))+(\uparrow \uparrow ; \downarrow \downarrow)+(\downarrow \downarrow ; \uparrow \uparrow)\right)=\frac{1}{\sqrt{2}}((\uparrow \uparrow)+(\downarrow \downarrow)) \frac{1}{\sqrt{2}}((\uparrow \uparrow)+(\downarrow \downarrow)) .
$$

The initial and final states are the third triplet state.

We can create a singlet state with $S=0$ according to

$$
\chi_{d}=\frac{1}{\sqrt{4}}\left(\chi_{\uparrow \uparrow ; \uparrow \uparrow}^{p h}+\chi_{\downarrow \downarrow ; \downarrow \downarrow}^{p h}-\chi_{\uparrow \uparrow ; \downarrow \downarrow}^{p h}-\chi_{\downarrow \downarrow ; \uparrow \uparrow}^{p h}\right) .
$$

We call this channel the density channel $d$. In the symbolic notation from above we obtain

$$
\frac{1}{\sqrt{4}}((\uparrow \uparrow ; \uparrow \uparrow)+(\downarrow \downarrow ; \downarrow)-(\uparrow \uparrow ; \downarrow \downarrow)-(\downarrow \downarrow ; \uparrow \uparrow))=\frac{1}{\sqrt{2}}((\uparrow \uparrow)-(\downarrow \downarrow)) \frac{1}{\sqrt{2}}((\uparrow \uparrow)-(\downarrow \downarrow)) .
$$

The initial state and final state have indeed a total $\operatorname{spin} S=0$.

We can now exploit the symmetry between spin up and down. Two channels that are related by flipping all spins are equal because of the spin symmetry. For the density channel $d$ and the magnetic channel $m^{0}$ we obtain

$$
\chi_{d / m^{0}}(\nu)_{\omega \omega^{\prime}}=\chi_{\uparrow \uparrow ; \uparrow \uparrow}^{p h}(\nu)_{\omega \omega^{\prime}} \pm \chi_{\uparrow \uparrow ; \downarrow \downarrow}^{p h}(\nu)_{\omega \omega^{\prime}} .
$$


Furthermore, SU(2) symmetry demands that $\chi_{m^{1}}=\chi_{m^{0}}=\chi_{m^{-1}}$. This reduces the number of independent particle-hole channels to two. In this thesis we work with $\chi_{d / m^{0}}$ and measure the two particle-hole Green functions on the right hand side of eq. (D.13).

For the particle-particle channel we have six possible spin configurations as well. These are $(\uparrow \uparrow ; \uparrow \uparrow),(\downarrow \downarrow ; \downarrow)),(\uparrow \downarrow ; \uparrow \downarrow),(\downarrow \uparrow ; \downarrow \uparrow),(\uparrow \downarrow ; \downarrow \uparrow)$ and $(\downarrow \uparrow ; \uparrow \downarrow)$. The first two have a total spin $S=1$ for the inital and final state, the last four have $s_{z}=0$ but they are no eigenstates of the square of the total spin operator.

Again, we can exploit symmetries to reduce the number of channels we have to measure. We start from the pairing channel Green function

$$
\chi_{p}(Q)_{\omega \omega^{\prime}}=\chi_{\uparrow \downarrow ; \uparrow \downarrow}^{p p}(Q)_{\omega \omega^{\prime}} .
$$

The corresponding diagram is shown in fig. D.2. The pairing channel is related to the singlet/triplet channel via

$$
\chi_{s / t}(\nu)_{\omega \omega^{\prime}}=\chi_{\uparrow \downarrow ; \uparrow \downarrow}^{p p}(\nu)_{\omega \omega^{\prime}} \mp \chi_{\uparrow \downarrow ; \downarrow \uparrow}^{p p}(\nu)_{\omega \omega^{\prime}}=\chi_{p}(\nu)_{\omega \omega^{\prime}} \pm \chi_{p}(\nu)_{\omega,-\omega^{\prime}-\nu}
$$

From the spin symmetry we get the $(\downarrow \uparrow ; \downarrow \uparrow)$ channel. The crossing symmetry relates the pairing channel with $\chi_{\uparrow \downarrow ; \downarrow \uparrow}^{p p}$. We proceeded the same way as we did for the particle-hole channel to obtain eq. (D.15) and find the singlet and triplet channels. In this case, we only have to measure the particle-particle Green function for the pairing channel.

The disorder two-particle Green function is defined via

$$
\chi_{v}(\nu)_{\omega \omega^{\prime}}=\chi_{v}^{=}(\nu)_{\omega \omega^{\prime}}=\left\{g_{\omega+\nu} g_{\omega}\right\}
$$

for the particle-hole channel. $\chi_{v}^{=}$refers to the horizontal channel. It is depicted in fig. D.3 on the left and the lowest order contribution to it is illustrated in fig. D.4. Here, $g$ is the impurity Green function. For the particle-particle channel it is calculated according to

$$
\chi_{v p}^{=}(\nu)_{\omega \omega^{\prime}}=\left\{g_{\omega+\nu} g_{-\omega}\right\}
$$

For the disorder two-particle Green function we omit the spin index as it does not depend on the spin configuration for the models we study. $\{\ldots\}$ denotes the disorder average, the two Green function lines in the diagrammatic representation of $\chi$ are connected through disorder scattering only. The lack of the vertical (crossed) component makes this Green function crossing-asymmetric. For the particle-hole disorder vertex we can construct the vertical component according to

$$
\chi_{v}^{\|}(\nu)_{\omega, \omega^{\prime}}=-\chi_{v}^{=}\left(\omega-\omega^{\prime}\right)_{\omega^{\prime}, \omega^{\prime}} \delta_{\nu, 0},
$$

which connects the horizontal channel with the vertical channel. The vertical chan- 
nel is unphysical, but can be convenient to use in some calculations. We find this

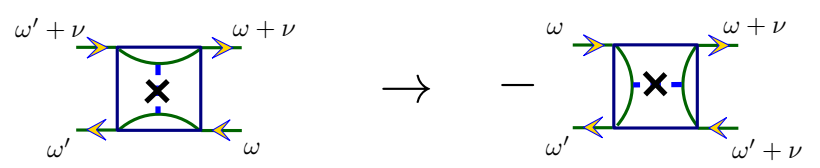

Figure D.3: Relation of the horizontal channel and the vertical channel for the disorder vertex.

relation from the crossing symmetry by switching the upper right corner of the twoparticle Green function with the lower left one as is shown in fig. D.3. For the particle-particle channel we relate the crossed channel to the horizontal one via

$$
\chi_{v p}^{\times}(\nu)_{\omega, \omega^{\prime}}=-\chi_{v p}^{=}(\nu)_{\omega,-\omega^{\prime}-\nu} \delta_{\omega+\omega+\nu, 0} .
$$

a)

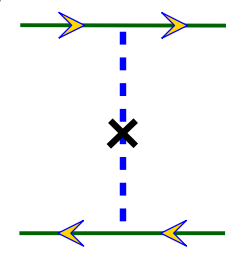

b)

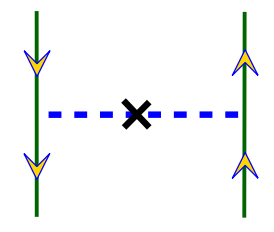

Figure D.4: In a) we have a contribution to the horizontal disorder channel, b) shows a contribution to the vertical disorder channel.

\section{D.2 Vertices}

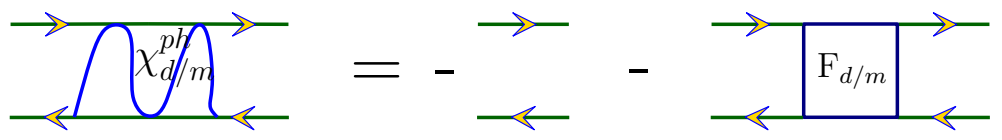

Figure D.5: The Bethe-Salpeter equation for the particle-hole channel.

The full vertex is obtained from the two-particle Green function via the BetheSalpeter equation

$$
\chi_{d / m}(\nu)_{\omega \omega^{\prime}}=-g(\omega+\nu) g(\omega) \delta_{\omega \omega^{\prime}}-g(\omega+\nu) g(\omega) F_{d / m} g\left(\omega^{\prime}+\nu\right) g\left(\omega^{\prime}\right)
$$

for the particle-hole channel and

$$
\chi_{p}(\nu)_{\omega \omega^{\prime}}=g(\omega+\nu) g(-\omega) \delta_{\omega \omega^{\prime}}-g(\omega+\nu) g(-\omega) F_{p g\left(\omega^{\prime}+\nu\right) g\left(-\omega^{\prime}\right)}
$$




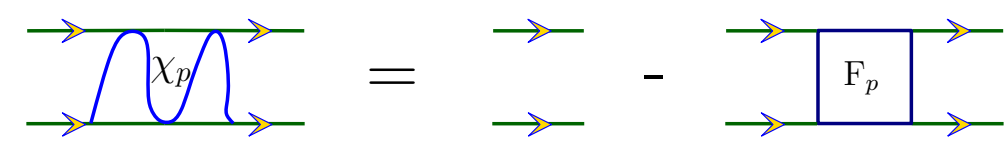

Figure D.6: The Bethe-Salpeter equation for the particle-particle channel.

for the particle-particle channel. It follows that we have to remove the disconnected part first, and then remove the four Green function legs by division.

For the particle-hole channel we obtain

$$
F_{d / m}(\nu)_{\omega \omega^{\prime}}=-\beta \frac{\chi_{d / m}(\nu)_{\omega \omega^{\prime}}+g_{\omega} g_{\omega+\nu} \delta_{\omega \omega^{\prime}}}{g_{\omega+\nu} g_{\omega} g_{\omega^{\prime}} g_{\omega^{\prime}+\nu}}
$$

The particle-particle vertex reads

$$
F_{p}(\nu)_{\omega \omega^{\prime}}=\beta \frac{-\chi_{p}(\nu)_{\omega \omega^{\prime}}+g_{\omega+\nu} g_{-\omega} \delta_{\omega \omega^{\prime}}}{g_{\omega+\nu} g_{-\omega} g_{-\omega^{\prime}} g_{\omega^{\prime}+\nu}}
$$

Finally, for the disorder vertex in the particle-hole channel we get

$$
F_{v}^{=}(\nu)_{\omega \omega^{\prime}}=-\beta \frac{\chi_{v}^{=}(\nu)_{\omega \omega}-g_{\omega} g_{\omega+\nu}}{g_{\omega+\nu} g_{\omega} g_{\omega} g_{\omega+\nu}} \delta_{\omega \omega^{\prime}}
$$

As a summary, in fig. D.7 all the vertices we need in this thesis are presented with their diagrammatic representation. Here, $F_{d / m / p}$ have been replaced in the diagrams by graphic symbols.

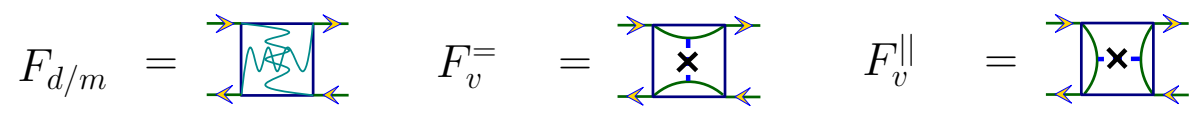

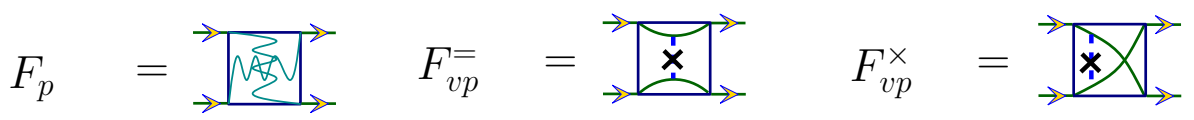

Figure D.7: Definition of the different vertices in terms of diagrammatic building blocks. The solid green lines for the disorder vertices indicate for which connection from corner to corner the energy is conserved. 


\section{E Comment on the Use of the Replica Trick}

In several discussions, the question was raised whether the replica trick is necessary to derive the dual fermion approach for disordered or disordered interacting systems or it was just stated that it should be. We use the replica trick to easily integrate out the disorder and for book-keeping purposes, that is to account for the correct diagrams and remove unphysical ones.

Alternatively, one could proceed to solve the lattice problem for a single disorder configuration and perform the disorder-average for the dual quantities. Before the disorder-average the vertex has only one contribution, namely from the interaction. For the purely disordered system the dual potential would be zero before the disorder-average.

Unlinked diagrams for the dual Green function would not appear, the same way as they do not appear for the real fermion Green function. Thus, the diagrams that contain what we call Fermi loops cannot be created. The disorder-average for the dual diagrams might be possible the same way that it can be done for the real fermion system [70] and it is thus possible to guess the correct equations.

Nevertheless, without an in-depth analysis the details of the disorder-average for the dual fermion system remain unclear. Practically, this is more involved as it may seem at a first glance. In the end, we found using the replica trick easier.

In that sense, it is pretty straight forward to see what the replica limit does for the diagrams and where it comes from. It is not as straight forward to actually do the derivation. 



\section{Bibliography}

[1] M. Born and R. Oppenheimer. Zur Quantentheorie der Molekeln. Annalen der Physik, 389(20):457-484, 1927.

[2] Steven R. White. Density matrix formulation for quantum renormalization groups. Phys. Rev. Lett., 69:2863-2866, Nov 1992.

[3] Gordon Baym and Christopher Pethick. Landau Fermi-Liquid Theory and Low Temperature Properties of Normal Liquid 3He, pages 1-121. Wiley-VCH Verlag GmbH, 2007.

[4] L. D. Landau. The theory of a Fermi liquid. Soviet Physics Jetp-Ussr, 3(6):920-925, 1957.

[5] Albert Messiah. Quantenmechanik, Band 2, chapter 16. Walter de Gruyter, Berlin - New York, 1990.

[6] Sang-Wook Cheong. Transition metal oxides: The exciting world of orbitals. Nat Mater, 6:927928, 122007.

[7] K. Andres, J. E. Graebner, and H. R. Ott. $4 f$-Virtual-Bound-State Formation in $\mathrm{CeAl}_{3}$ at Low Temperatures. Phys. Rev. Lett., 35:1779-1782, Dec 1975.

[8] F. Steglich, J. Aarts, C. D. Bredl, W. Lieke, D. Meschede, W. Franz, and H. Schäfer. Superconductivity in the Presence of Strong Pauli Paramagnetism: $\mathrm{CeCu}_{2} \mathrm{Si}_{2}$. Phys. Rev. Lett., 43:1892-1896, Dec 1979.

[9] H. v. Löhneysen, T. Pietrus, G. Portisch, H. G. Schlager, A. Schröder, M. Sieck, and T. Trappmann. Non-Fermi-liquid behavior in a heavy-fermion alloy at a magnetic instability. Phys. Rev. Lett., 72:3262-3265, May 1994.

[10] Qimiao Si and Frank Steglich. Heavy Fermions and Quantum Phase Transitions. Science, 329(5996):1161-1166, 2010.

[11] G. R. Stewart. Heavy-fermion systems. Rev. Mod. Phys., 56:755-787, Oct 1984.

[12] Elbio Dagotto. Complexity in Strongly Correlated Electronic Systems. Science, 309(5732):257-262, 2005. 
[13] D. B. McWhan, A. Menth, J. P. Remeika, W. F. Brinkman, and T. M. Rice. Metal-Insulator Transitions in Pure and Doped $\mathrm{V}_{2} \mathrm{O}_{3}$. Phys. Rev. B, 7:19201931, Mar 1973.

[14] Masatoshi Imada, Atsushi Fujimori, and Yoshinori Tokura. Metal-insulator transitions. Rev. Mod. Phys., 70(4):1039-1263, Oct 1998.

[15] Jan M. Tomczak and Silke Biermann. Optical properties of correlated materials - Or why intelligent windows may look dirty. physica status solidi (b), 246(9):1996-2005, 2009.

[16] P. W. Anderson. Absence of Diffusion in Certain Random Lattices. Phys. Rev., 109:1492-1505, Mar 1958.

[17] Alexander D. Mirlin. Statistics of energy levels and eigenfunctions in disordered systems. Physics Reports, 326(5 - 6):259 - 382, 2000.

[18] Michael Schreiber and Heiko Grussbach. Multifractal wave functions at the Anderson transition. Phys. Rev. Lett., 67:607-610, Jul 1991.

[19] Patrick A. Lee and T. V. Ramakrishnan. Disordered electronic systems. Rev. Mod. Phys., 57:287-337, Apr 1985.

[20] D. Belitz and T. R. Kirkpatrick. The Anderson-Mott transition. Rev. Mod. Phys., 66:261-380, Apr 1994.

[21] J. Hubbard. Electron Correlations in Narrow Energy Bands. Proceedings of the Royal Society of London. Series A. Mathematical and Physical Sciences, 276(1365):238-257, 1963.

[22] Junjiro Kanamori. Electron Correlation and Ferromagnetism of Transition Metals. Progress of Theoretical Physics, 30(3):275-289, 1963.

[23] Martin C. Gutzwiller. Effect of Correlation on the Ferromagnetism of Transition Metals. Phys. Rev. Lett., 10(5):159-162, Mar 1963.

[24] M. Ulmke, V. Janišs, and D. Vollhardt. Anderson-Hubbard model in infinite dimensions. Phys. Rev. B, 51:10411-10426, Apr 1995.

[25] Emanuel Gull, Andrew J. Millis, Alexander I. Lichtenstein, Alexey N. Rubtsov, Matthias Troyer, and Philipp Werner. Continuous-time Monte Carlo methods for quantum impurity models. Rev. Mod. Phys., 83(2):349-404, May 2011.

[26] Walter Metzner and Dieter Vollhardt. Correlated Lattice Fermions in $d=\infty$ Dimensions. Phys. Rev. Lett., 62:324-327, Jan 1989. 
[27] E. Müller-Hartmann. Correlated fermions on a lattice in high dimensions. $Z$. Phys., 74:507-512, 1989.

[28] T. Pruschke, M. Jarrell, and J. K. Freericks. Anomalous normal-state properties of high- $\mathrm{T}_{c}$ superconductors: intrinsic properties of strongly correlated electron systems? Adv. in Phys., 44:187, 1995.

[29] Antoine Georges, Gabriel Kotliar, Werner Krauth, and Marcelo J. Rozenberg. Dynamical mean-field theory of strongly correlated fermion systems and the limit of infinite dimensions. Rev. Mod. Phys., 68(1):13, Jan 1996.

[30] V. Janiš and D. Vollhardt. Coupling of quantum degrees of freedom in strongly interacting disordered electron systems. Phys. Rev. B, 46:15712-15715, Dec 1992.

[31] V. Dobrosavljević and G. Kotliar. Strong correlations and disorder in $d=\infty$ and beyond. Phys. Rev. B, 50:1430-1449, Jul 1994.

[32] M. H. Hettler, A. N. Tahvildar-Zadeh, M. Jarrell, T. Pruschke, and H. R. Krishnamurthy. Nonlocal dynamical correlations of strongly interacting electron systems. Phys. Rev. B, 58:R7475-R7479, Sep 1998.

[33] M. H. Hettler, M. Mukherjee, M. Jarrell, and H. R. Krishnamurthy. Dynamical cluster approximation: Nonlocal dynamics of correlated electron systems. Phys. Rev. B, 61:12739-12756, May 2000.

[34] Gabriel Kotliar, Sergej Y. Savrasov, Gunnar Pálsson, and Giulio Biroli. Cellular Dynamical Mean Field Approach to Strongly Correlated Systems. Phys. Rev. Lett., 87:186401, Oct 2001.

[35] V. Janiš. Parquet approach to nonlocal vertex functions and electrical conductivity of disordered electrons. Phys. Rev. B, 64:115115, Aug 2001.

[36] A. N. Rubtsov, M. I. Katsnelson, and A. I. Lichtenstein. Dual fermion approach to nonlocal correlations in the Hubbard model. Phys. Rev. B, 77:033101, Jan 2008.

[37] A. N. Rubtsov, M. I. Katsnelson, A. I. Lichtenstein, and A. Georges. Dual fermion approach to the two-dimensional Hubbard model: Antiferromagnetic fluctuations and Fermi arcs. Phys. Rev. B, 79:045133, Jan 2009.

[38] K. Held, A. A. Katanin, and A. Toschi. Dynamical vertex approximation an introduction. arXiv:080\%.1860, Jul 2008.

[39] A. Toschi, A. A. Katanin, and K. Held. Dynamical vertex approximation: A step beyond dynamical mean-field theory. Phys. Rev. B, 75:045118, Jan 2007. 
[40] H. Terletska, S.-X. Yang, Z. Y. Meng, J. Moreno, and M. Jarrell. Dual fermion method for disordered electronic systems. Phys. Rev. B, 87:134208, Apr 2013.

[41] Krzysztof Byczuk. Metal-insulator transitions in the Falicov-Kimball model with disorder. Phys. Rev. B, 71:205105, May 2005.

[42] A. N. Rubtsov and A. I. Lichtenstein. Continuous-time quantum Monte Carlo method for fermions: Beyond auxiliary field framework. JETP Letters, 80:6165, 072004.

[43] A. N. Rubtsov, V. V. Savkin, and A. I. Lichtenstein. Continuous-time quantum Monte Carlo method for fermions. Phys. Rev. B, 72(3):035122, Jul 2005.

[44] F. F. Assaad and T. C. Lang. Diagrammatic determinantal quantum Monte Carlo methods: Projective schemes and applications to the Hubbard-Holstein model. Phys. Rev. B, 76(3):035116, Jul 2007.

[45] L. M. Falicov and J. C. Kimball. Simple Model for Semiconductor-Metal Transitions: $\mathrm{SmB}_{6}$ and Transition-Metal Oxides. Phys. Rev. Lett., 22:997999, May 1969.

[46] D.R. Hartree. The wave mechanics of an atom with a non-Coulomb central field. Proc. Cambridge Phil. Soc., 24(1-2):89-132, 1928.

[47] V. Fock. Näherungsmethode zur Lösung des quantenmechanischen Mehrkörperproblems. Zeitschrift für Physik, 61(1-2):126-148, 1930.

[48] N.W. Ashcroft and N.D. Mermin. Solid State Physics. Saunders College Publishing, 1976.

[49] N F Mott. The Basis of the Electron Theory of Metals, with Special Reference to the Transition Metals. Proceedings of the Physical Society. Section A, 62(7):416, 1949 .

[50] João Florencio and K. A. Chao. Theory of itinerant antiferromagnetism: Zerotemperature properties. Phys. Rev. B, 14:3121-3129, Oct 1976.

[51] C. L. Seaman, M. B. Maple, B. W. Lee, S. Ghamaty, M. S. Torikachvili, J.-S. Kang, L. Z. Liu, J. W. Allen, and D. L. Cox. Evidence for non-Fermi liquid behavior in the Kondo alloy $\mathrm{Y}_{1-x} \mathrm{U}_{x} \mathrm{Pd}_{3}$. Phys. Rev. Lett., 67:2882-2885, Nov 1991.

[52] C. Castellani, C. Di Castro, and W. Metzner. Dimensional crossover from Fermi to Luttinger liquid. Phys. Rev. Lett., 72:316-319, Jan 1994. 
[53] Jun Kondo. Resistance Minimum in Dilute Magnetic Alloys. Progress of Theoretical Physics, 32(1):37-49, 1964.

[54] Tadao Kasuya. A Theory of Metallic Ferro- and Antiferromagnetism on Zener's Model. Progress of Theoretical Physics, 16(1):45-57, 1956.

[55] S. Doniach. Phase Diagram for the Kondo Lattice. In R.D. Parks, editor, Valence Instabilities and Related Narrow-Band Phenomena, pages 169-176. Springer US, 1977.

[56] Elliott H. Lieb and F. Y. Wu. Absence of Mott Transition in an Exact Solution of the Short-Range, One-Band Model in One Dimension. Phys. Rev. Lett., 20:1445-1448, Jun 1968.

[57] N. F. Mott. Metal-insulator transitions. Taylor and Francis, London, 1974.

[58] Hal Tasaki. The Hubbard model - an introduction and selected rigorous results. Journal of Physics: Condensed Matter, 10(20):4353, 1998.

[59] J. P. F. LeBlanc, Andrey E. Antipov, Federico Becca, Ireneusz W. Bulik, Garnet Kin-Lic Chan, Chia-Min Chung, Youjin Deng, Michel Ferrero, Thomas M. Henderson, Carlos A. Jiménez-Hoyos, E. Kozik, Xuan-Wen Liu, Andrew J. Millis, N. V. Prokof'ev, Mingpu Qin, Gustavo E. Scuseria, Hao Shi, B. V. Svistunov, Luca F. Tocchio, I. S. Tupitsyn, Steven R. White, Shiwei Zhang, Bo-Xiao Zheng, Zhenyue Zhu, and Emanuel Gull. Solutions of the Two Dimensional Hubbard Model: Benchmarks and Results from a Wide Range of Numerical Algorithms. arXiv:1505.02290, 2015.

[60] Alexander Weiße and Holger Fehske. Exact diagonalization techniques. In H. Fehske, R. Schneider, and A. Weiße, editors, Computational Many-Particle Physics, volume 739 of Lecture Notes in Physics, pages 529-544. Springer Berlin Heidelberg, 2008.

[61] Thomas Maier, Mark Jarrell, Thomas Pruschke, and Matthias H. Hettler. Quantum cluster theories. Rev. Mod. Phys., 77:1027-1080, Oct 2005.

[62] Tom Kennedy and Elliott H. Lieb. An itinerant electron model with crystalline or magnetic long range order. Physica A: Statistical Mechanics and its Applications, 138(1 - 2):320 - 358, 1986.

[63] U. Brandt and R. Schmidt. Exact results for the distribution of the f-level ground state occupation in the spinless Falicov-Kimball model. Zeitschrift für Physik B Condensed Matter, 63(1):45-53, 1986.

[64] J. K. Freericks and V. Zlatić. Exact dynamical mean-field theory of the Falicov-Kimball model. Rev. Mod. Phys., 75:1333-1382, Oct 2003. 
[65] Paul Soven. Coherent-Potential Model of Substitutional Disordered Alloys. Phys. Rev., 156:809-813, Apr 1967.

[66] D. W. Taylor. Vibrational Properties of Imperfect Crystals with Large Defect Concentrations. Phys. Rev., 156:1017-1029, Apr 1967.

[67] Michael Plischke. Coherent-Potential-Approximation Calculation on the Falicov-Kimball Model of the Metal-Insulator Transition. Phys. Rev. Lett., 28:361-363, Feb 1972.

[68] A. Cavalleri. Chemical physics: Melted in a flash. Nature, 458:42-43, 32009.

[69] J Lian, L. M. Wang, and R. C. Ewing. In Situ TEM Study of Order-Disorder Transition in Murataite Ceramics. Microscopy and Microanalysis, 8:14241425, 82002 .

[70] H. Bruus and K. Flensberg. Many-Body Quantum Theory in Condensed Matter Physics. Oxford University Press, 2004.

[71] S. V. Kravchenko, G. V. Kravchenko, J. E. Furneaux, V. M. Pudalov, and M. D'Iorio. Possible metal-insulator transition at $B=0$ in two dimensions. Phys. Rev. B, 50:8039-8042, Sep 1994.

[72] S V Kravchenko and M P Sarachik. Metal-insulator transition in twodimensional electron systems. Reports on Progress in Physics, 67(1):1, 2004.

[73] George Kastrinakis. Metal-insulator transition in 2D: the role of interactions and disorder. Physica B: Condensed Matter, 387(1 - 2):109 - 116, 2007.

[74] Jian-Feng Ge, Zhi-Long Liu, Canhua Liu, Chun-Lei Gao, Dong Qian, Qi-Kun Xue, Ying Liu, and Jin-Feng Jia. Superconductivity above $100 \mathrm{~K}$ in singlelayer FeSe films on doped $\mathrm{SrTiO}_{3}$. Nat Mater, 14:285289, 32015.

[75] Madhavi Chand, Archana Mishra, Y. M. Xiong, Anand Kamlapure, S. P. Chockalingam, John Jesudasan, Vivas Bagwe, Mintu Mondal, P. W. Adams, Vikram Tripathi, and Pratap Raychaudhuri. Temperature dependence of resistivity and Hall coefficient in strongly disordered NbN thin films. Phys. Rev. $B, 80: 134514$, Oct 2009 .

[76] R. Schneider, A. G. Zaitsev, D. Fuchs, and H. v. Löhneysen. SuperconductorInsulator Quantum Phase Transition in Disordered FeSe Thin Films. Phys. Rev. Lett., 108:257003, Jun 2012.

[77] O. Crauste, A. Gentils, F. Couëdo, Y. Dolgorouky, L. Bergé, S. Collin, C. A. Marrache-Kikuchi, and L. Dumoulin. Effect of annealing on the superconducting properties of $a-\mathrm{Nb}_{x} \mathrm{Si}_{1-x}$ thin films. Phys. Rev. B, 87:144514, Apr 2013. 
[78] P. W. Anderson. Localized Magnetic States in Metals. Phys. Rev., 124(1):4153, Oct 1961.

[79] Alexander C. Hewson. The Kondo Problem to Heavy Fermions (Cambridge Studies in Magnetism). Cambridge University Press, April 1997.

[80] H. Yukawa. On the Interaction of Elementary Particles. Proc.Phys.Math.Soc.Jap., 17:48, 1935.

[81] Ta-Feng Lin. Problem of the Disordered Chain. Journal of Mathematical Physics, 11(5):1584-1590, 1970.

[82] Mark Kac. On certain Toeplitz-like matrices and their relation to the problem of lattice vibrations. Arkiv for det Fysiske seminar $i$ Trondheim, 1978.

[83] Tommaso Castellani and Andrea Cavagna. Spin-glass theory for pedestrians. Journal of Statistical Mechanics: Theory and Experiment, 2005(05):P05012, 2005 .

[84] M. Jarrell and H. R. Krishnamurthy. Systematic and causal corrections to the coherent potential approximation. Phys. Rev. B, 63:125102, Mar 2001.

[85] T. Schäfer, F. Geles, D. Rost, G. Rohringer, E. Arrigoni, K. Held, N. Blümer, M. Aichhorn, and A. Toschi. Fate of the false Mott-Hubbard transition in two dimensions. Phys. Rev. B, 91:125109, Mar 2015.

[86] V. Dobrosavljević, A. A. Pastor, and B. K. Nikolić. Typical medium theory of Anderson localization: A local order parameter approach to strong-disorder effects. EPL (Europhysics Letters), 62(1):76, 2003.

[87] Th. A. Maier and M. Jarrell. Zinc Impurities in $d$-Wave Superconductors. Phys. Rev. Lett., 89:077001, Jul 2002.

[88] A. F. Kemper, D. G. S. P. Doluweera, T. A. Maier, M. Jarrell, P. J. Hirschfeld, and H-P. Cheng. Insensitivity of $d$-wave pairing to disorder in the hightemperature cuprate superconductors. Phys. Rev. B, 79:104502, Mar 2009.

[89] M. Jarrell. Hubbard model in infinite dimensions: A quantum Monte Carlo study. Phys. Rev. Lett., 69:168-171, Jul 1992.

[90] Antoine Georges and Werner Krauth. Numerical solution of the $d=\infty$ Hubbard model: Evidence for a Mott transition. Phys. Rev. Lett., 69:1240-1243, Aug 1992. 
[91] Chinedu E Ekuma, Hanna Terletska, Zi Yang Meng, Juana Moreno, Mark Jarrell, Samiyeh Mahmoudian, and Vladimir Dobrosavljević. Effective cluster typical medium theory for the diagonal Anderson disorder model in one- and two-dimensions. Journal of Physics: Condensed Matter, 26(27):274209, 2014.

[92] C. E. Ekuma, H. Terletska, K.-M. Tam, Z.-Y. Meng, J. Moreno, and M. Jarrell. Typical medium dynamical cluster approximation for the study of Anderson localization in three dimensions. Phys. Rev. B, 89:081107, Feb 2014.

[93] M. Potthoff, T. Wegner, and W. Nolting. Interpolating self-energy of the infinite-dimensional Hubbard model: Modifying the iterative perturbation theory. Phys. Rev. B, 55(24):16132-16142, Jun 1997.

[94] Armin Comanac. Dynamical mean field theory of correlated electron systems: New algorithms and application to local observables. PhD thesis, Columbia University, New York, 2007.

[95] R. Mills and P. Ratanavararaksa. Analytic approximation for substitutional alloys. Phys. Rev. B, 18(10):5291-5308, November 1978.

[96] T. Kaplan, P. L. Leath, L. J. Gray, and H. W. Diehl. Self-consistent cluster theory for systems with off-diagonal disorder. Phys. Rev. B, 21:4230, 1980.

[97] M. Tsukada. A New Method for the Electronic Structure of Random Lattice -the Coexistence of the Local and the Band Character-. J. Phys. Soc. Jpn., 26:684-696, 1969.

[98] M. Tsukada. Contribution to the Many Site Theory of the Disordered System. J. Phys. Soc. Jpn., 32:1475, 1972.

[99] F. Ducastelle. Analytic properties of the coherent potential approximation and of its molecular generalization. J. Phys. C Sol. State Phys., 7:1795-1816, 1974 .

[100] A. Mookerjee. A new formalism for the study of configuration-averaged properties of disordered systems. J. Phys. C: Solid State Phys., 6:L205, 1973.

[101] T. Kaplan and L. J. Gray. Elementary excitations in random substitutional alloys. Phys. Rev. B, 14:3462, 1976.

[102] T. Kaplan and L. J. Gray. Off-diagonal disorder in random substitutional alloys. J. Phys. C: Solid State Phys., 9:L303, 1976.

[103] V. Kumar, A. Mookerjee, and V. K. Srivastava. Electronic structure of disordered alloys I. Self-consistent cluster CPA incorporating off-diagonal disorder and short-range order. J. Phys. C: Solid State Phys., 15:1939, 1982. 
[104] A. Mookerjee. Cluster generalisation of the KKR coherent potential methods for random metallic alloys. J. Phys. F: Met. Phys., 17:1511, 1987.

[105] H. Hafermann. Numerical Approaches to Spatial Correlations in Strongly Interacting Fermion Systems. Cuvillier Verlag, Göttingen, 2010.

[106] S.-X. Yang, P. Haase, H. Terletska, Z. Y. Meng, T. Pruschke, J. Moreno, and M. Jarrell. Dual-fermion approach to interacting disordered fermion systems. Phys. Rev. B, 89:195116, May 2014.

[107] J. W. Negele and H. Orland. Quantum Many-Particle Systems. Addison Wesley, 1988.

[108] R. D. Mattuck. A Guide to Feynman Diagrams in the Many-Body Problem. The McGraw-Hill Book Company, New York, 1976.

[109] Anil Khurana. Electrical conductivity in the infinite-dimensional Hubbard model. Phys. Rev. Lett., 64:1990-1990, Apr 1990.

[110] S. X. Yang, H. Fotso, J. Liu, T. A. Maier, K. Tomko, E. F. D’Azevedo, R. T. Scalettar, T. Pruschke, and M. Jarrell. Parquet approximation for the $4 \times 4$ Hubbard cluster. Phys. Rev. E, 80:046706, Oct 2009.

[111] Nandini Trivedi, Richard T. Scalettar, and Mohit Randeria. Superconductorinsulator transition in a disordered electronic system. Phys. Rev. B, 54:R3756R3759, Aug 1996.

[112] S. Brener, H. Hafermann, A. N. Rubtsov, M. I. Katsnelson, and A. I. Lichtenstein. Dual fermion approach to susceptibility of correlated lattice fermions. Phys. Rev. B, 77:195105, May 2008.

[113] Kenneth G. Wilson. The renormalization group: Critical phenomena and the Kondo problem. Rev. Mod. Phys., 47:773-840, Oct 1975.

[114] Ralf Bulla, Theo A. Costi, and Thomas Pruschke. Numerical renormalization group method for quantum impurity systems. Rev. Mod. Phys., 80:395-450, Apr 2008.

[115] Ansgar Liebsch and Hiroshi Ishida. Temperature and bath size in exact diagonalization dynamical mean field theory. Journal of Physics: Condensed Matter, 24(5):053201, 2012.

[116] C. Lanczos. An Iteration Method for the Solution of the Eigenvalue Problem of Linear Differential and Intergral Operators. J. Res. Nat. Bur. Stand., 45:25582,1950 . 
[117] Gerard L. G. Sleijpen and Henk A. van der Vorst. A Jacobi-Davidson Iteration Method for Linear Eigenvalue Problems. SIAM J. Matrix Anal. Appl., 17(2):401-425, April 1996.

[118] S. A. Jafari. Introduction to Hubbard Model and Exact Diagonalization. arXiv:080\%.4878, 2008.

[119] Elbio Dagotto. Correlated electrons in high-temperature superconductors. Rev. Mod. Phys., 66:763-840, Jul 1994.

[120] Martin Ganahl, Patrik Thunström, Frank Verstraete, Karsten Held, and Hans Gerd Evertz. Chebyshev expansion for impurity models using matrix product states. Phys. Rev. B, 90:045144, Jul 2014.

[121] F. Alexander Wolf, Ian P. McCulloch, and Ulrich Schollwöck. Solving nonequilibrium dynamical mean-field theory using matrix product states. Phys. Rev. B, 90:235131, Dec 2014 .

[122] F. Alexander Wolf, Jorge A. Justiniano, Ian P. McCulloch, and Ulrich Schollwöck. Spectral functions and time evolution from the Chebyshev recursion. Phys. Rev. B, 91:115144, Mar 2015.

[123] J. E. Hirsch and R. M. Fye. Monte Carlo Method for Magnetic Impurities in Metals. Phys. Rev. Lett., 56:2521-2524, Jun 1986.

[124] Philipp Werner, Armin Comanac, Luca de' Medici, Matthias Troyer, and Andrew J. Millis. Continuous-Time Solver for Quantum Impurity Models. Phys. Rev. Lett., 97:076405, Aug 2006.

[125] E. Gull, P. Werner, O. Parcollet, and M. Troyer. Continuous-time auxiliaryfield Monte Carlo for quantum impurity models. EPL (Europhysics Letters), 82(5):57003, 2008.

[126] Carey Huscroft, Richard Gass, and Mark Jarrell. Maximum entropy method of obtaining thermodynamic properties from quantum Monte Carlo simulations. Phys. Rev. B, 61(14):9300-9306, Apr 2000.

[127] Philipp Werner, Takashi Oka, and Andrew J. Millis. Diagrammatic Monte Carlo simulation of nonequilibrium systems. Phys. Rev. B, 79:035320, Jan 2009.

[128] Emanuel Gull, David R. Reichman, and Andrew J. Millis. Numerically exact long-time behavior of nonequilibrium quantum impurity models. Phys. Rev. B, 84:085134, Aug 2011. 
[129] Aicke Hinrichs, Erich Novak, Mario Ullrich, and Henryk Woźniakowski. The curse of dimensionality for numerical integration of smooth functions II. Journal of Complexity, 30(2):117 - 143, 2014. Dagstuhl 2012.

[130] N. Metropolis, A.W. Rosenbluth, M.N. Rosenbluth, A.H. Teller, and E. Teller. Equation of State Calculations by Fast Computing Machines. The Journal of Chemical Physcis, 21:1087-1092, 1953.

[131] W. H. Press, B. P. Flannery, S. A. Teukolsky, and W. T. Vetterling. Numerical Recipes in Fortran 77: The Art of Scientific Computing. University Press, Cambridge, 1992.

[132] W. K. Hastings. Monte Carlo sampling methods using Markov chains and their applications. Biometrika, 57(1):97-109, 1970.

[133] G. J. Daniell, A. J. G. Hey, and J. E. Mandula. Error analysis for correlated Monte Carlo data. Phys. Rev. D, 30:2230-2232, Nov 1984.

[134] A. M. Davie and A. J. Stothers. Improved bound for complexity of matrix multiplication. Proceedings of the Royal Society of Edinburgh, Section: A Mathematics, 143:351-369, 42013.

[135] Andrey E. Antipov, Emanuel Gull, and Stefan Kirchner. Critical Exponents of Strongly Correlated Fermion Systems from Diagrammatic Multiscale Methods. Phys. Rev. Lett., 112:226401, Jun 2014.

[136] M. A. Gusmão. Phase diagram of the Anderson-Falicov-Kimball model at half filling. Phys. Rev. B, 77:245116, Jun 2008.

[137] D. Semmler, K. Byczuk, and W. Hofstetter. Anderson-Hubbard model with box disorder: Statistical dynamical mean-field theory investigation. Phys. Rev. B, 84:115113, Sep 2011.

[138] C Slezak, M Jarrell, Th Maier, and J Deisz. Multi-scale extensions to quantum cluster methods for strongly correlated electron systems. Journal of Physics: Condensed Matter, 21(43):435604, 2009.

[139] G. D. Mahan. Many Particle Physics, Third Edition. Plenum, New York, 2000 .

[140] P. R. C. Kent, M. Jarrell, T. A. Maier, and Th. Pruschke. Efficient calculation of the antiferromagnetic phase diagram of the three-dimensional Hubbard model. Phys. Rev. B, 72:060411, Aug 2005. 
[141] Daniel Hirschmeier, Hartmut Hafermann, Emanuel Gull, Alexander I. Lichtenstein, and Andrey E. Antipov. Mechanisms of finite temperature magnetism in the three-dimensional Hubbard model. arXiv:150\%.00616, Jul 2015.

[142] Sebastian Fuchs. Thermodynamic and spectral properties of quantum manyparticle systems. Dissertation, Georg-August-Universität Göttingen, 2011.

[143] Matthias Balzer and Michael Potthoff. Disorder- and correlation-driven metalinsulator transitions. Physica B: Condensed Matter, 359-361(0):768 - 770, 2005. Proceedings of the International Conference on Strongly Correlated Electron Systems.

[144] C. E. Ekuma, C. Moore, H. Terletska, K.-M. Tam, J. Moreno, M. Jarrell, and N. S. Vidhyadhiraja. Finite-cluster typical medium theory for disordered electronic systems. Phys. Rev. B, 92:014209, Jul 2015.

[145] Krzysztof Byczuk, Walter Hofstetter, and Dieter Vollhardt. Mott-Hubbard Transition versus Anderson Localization in Correlated Electron Systems with Disorder. Phys. Rev. Lett., 94:056404, Feb 2005.

[146] Maciej M. Maśka and Katarzyna Czajka. Pattern formation in the FalicovKimball model. physica status solidi (b), 242(2):479-483, 2005.

[147] R. Staudt, M. Dzierzawa, and A. Muramatsu. Phase diagram of the threedimensional Hubbard model at half filling. The European Physical Journal B - Condensed Matter and Complex Systems, 17(3):411-415, 2000.

[148] C. E. Ekuma, C. Moore, H. Terletska, K.-M. Tam, N. S. Vidhyadhiraja, J. Moreno, and M. Jarrell. Finite Cluster Typical Medium Theory for Disordered Electronic Systems. arXiv:1505.02825, 2015.

[149] P. Hohenberg and W. Kohn. Inhomogeneous Electron Gas. Phys. Rev., 136:B864-B871, Nov 1964.

[150] W. Kohn and L. J. Sham. Self-Consistent Equations Including Exchange and Correlation Effects. Phys. Rev., 140:A1133-A1138, Nov 1965.

[151] H. Shishido, T. Shibauchi, K. Yasu, T. Kato, H. Kontani, T. Terashima, and Y. Matsuda. Tuning the Dimensionality of the Heavy Fermion Compound $\mathrm{CeIn}_{3}$. Science, 327(5968):980-983, 2010.

[152] Yasuhiro Tada, Robert Peters, and Masaki Oshikawa. Dimensional crossover in layered $f$-electron superlattices. Phys. Rev. B, 88:235121, Dec 2013.

[153] S.-X. Yang. Next Generation Multi-Scale Quantum Simulations for Strongly Correlated Materials. Dissertation, Louisiana State University, 2011. 


\section{Acknowledgements}

Im Laufe meiner Promotion habe ich von vielen verschiedenen Personen immer wieder Unterstützung erhalten. Zuerst möchte ich mich dafür bei meinem Doktorvater Thomas Pruschke bedanken. Er hat mir während meiner Promotion die Freiheit gelassen, eigene Ideen und Lösungsansätze zu verfolgen, aber mir auch immer dann geholfen und mich in die richtige Richtung gelenkt, wenn ich nicht weiter wusste. Ebenso möchte ich meinem zweiten Betreuer Reiner Kree danken, sowohl für inhaltliche Diskussionen und Feedback zu meiner Arbeit, wie auch zu formalen Fragen im Zusammenhang mit meiner Promotion.

Weiterhin möchte ich den Mitgliedern der Festkörpertheorie für die angenehme Atmosphäre in dieser Gruppe danken, insbesondere denjenigen, die meine Arbeit gelesen und mir mit ihren Rückmeldungen geholfen haben, meine Ergebnisse möglichst verständlich und klar zu präsentieren. Namentlich möchte ich insbesondere Rainer Härtle, Florian Sohn und Benjamin Lenz erwähnen.

Ebenso danke ich Silke Teschke dafür, dass sie meine Arbeit im Hinblick auf sprachliche Richtigkeit korrekturgelesen hat.

Special thanks goes to Shuxiang Yang from the Louisiana State University (LSU). Shuxiang supervised me on the whole project on disordered interacting systems. He helped me learn the necessary tools to address this topic and supported me along the way in our weekly discussions. I also thank Mark Jarrell and Juana Moreno from LSU for useful discussions and for having me on the dual fermion project.

Meinen Eltern danke ich für ihre fortwährende Unterstützung, sowie dafür, dass sie mir den Wert von Bildung vermittelt haben. Zum Schluss danke ich Merle für ihre Unterstützung und aufmunternde Worte, wenn es mal nicht so gut lief.

I acknowledge financial support from the SFB 602, FOR 1807 as well as funding by the National Science Foundation under the NSF EPSCoR Cooperative Agreement No. EPS-1003897 with additional support from the Louisiana Board of Regents. 



\section{Curriculum Vitae}

\section{Persönliche Daten}

Name:

Patrick Haase

Geburtsdatum:

Geburtsort:

Staatsangehörigkeit:
05. April 1985

Soltau, Deutschland

deutsch

\section{Bildung}

Jan. 2014-März 2014: Aufenthalt als "Short Term Scholar" an der Louisiana State University, Baton Rouge, USA in der Arbeitsgruppe von Prof. Mark Jarrell, PhD.

Seit Apr. 2011: Promotionsstudium an der Georg-August-Universität Göttingen, Betreuer: Prof. Dr. Thomas Pruschke und Prof. Dr. Reiner Kree

März 2011: $\quad$ Abschluss mit dem Titel "Diplom-Physiker" (Note: sehr gut), Titel der Diplomarbeit: The Hubbard-Holstein Model with Dispersive Phonons and Graphene with an Impurity, Betreuer: Prof. Dr. Thomas Pruschke

Sept. 2010-Nov. 2010: Arbeit in der Gruppe von Dr. Francisco Guinea am ICMM-CSIC in Madrid

Febr. 2009-Juni 2009: Studienaufenthalt an der Universidad Autónoma de Madrid 2005-2011: Studium an der Georg-August-Universität Göttingen, Studiengang: Physik

Juni 2004: Gymnasium Walsrode, Allgemeine Hochschulreife (Note: 2,0) 


\section{Publikation im Zusammenhang mit dieser Arbeit}

S.-X. Yang, P. Haase, H. Terletska, Z. Y. Meng, T. Pruschke, J. Moreno, and M. Jarrell. Dual-fermion approach to interacting disordered fermion systems. Phys. Rev. $B, 89: 195116$, May 2014

\section{Sonstige Publikationen}

P. Haase, S. Fuchs, T. Pruschke, H. Ochoa, and F. Guinea. Magnetic moments and Kondo effect near vacancies and resonant scatterers in graphene. Phys. Rev. B, 83:241408, Jun 2011 